\title{
THERMODYNAMIC PROPERTIES: ENTHALPY, ENTROPY, HEAT CAPACITY AND BOND ENERGIES OF FLUORINATED CARBOXYLIC ACIDS
}

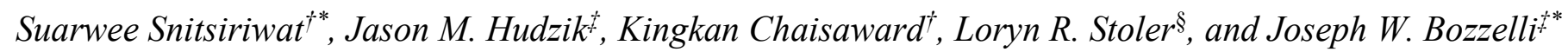

Department of Chemistry, Faculty of Science, Mahidol University, 272 Rama VI Road, Ratchathewi, Bangkok 10400, Thailand

${ }^{\S}$ Department of Chemistry and Biochemistry, University of Maryland, Baltimore, Maryland 21250, United States

${ }^{\ddagger}$ Chemistry, Chemical Engineering and Environmental Science, New Jersey Institute of Technology, Newark, New Jersey 07102, United States

\section{Supporting Information}

\begin{tabular}{lc} 
Section & Page \\
\hline Optimized Structures & $\mathrm{S} 2$ \\
Vibrational Frequencies & $\mathrm{S} 8$ \\
Moments of Inertia & $\mathrm{S} 11$ \\
Hindered Internal Rotor Potential Energy Diagrams & $\mathrm{S} 13$ \\
SMCPs input files & $\mathrm{S} 26$ \\
List of available literature data & $\mathrm{S} 29$ \\
Uncertainty at 95\% confidence limit & $\mathrm{S} 32$ \\
Work reactions & $\mathrm{S} 35$ \\
Nomenclature of Chemical Compounds & $\mathrm{S} 61$ \\
Parameters for the Rotator & $\mathrm{S} 63$ \\
Complete References & $\mathrm{S} 71$
\end{tabular}




\section{Optimized Structures}

In this article, explicit hydrogens are not included in species but are implied unless otherwise stated.And $\mathrm{j}$ represents a radical site on the preceding carbon or oxygen atom.

\section{From MN15/cc-pVTZ level of theory}

\section{$\mathrm{CF}-\mathrm{C}(=\mathbf{0}) \mathrm{OH}(\sigma=1)$}

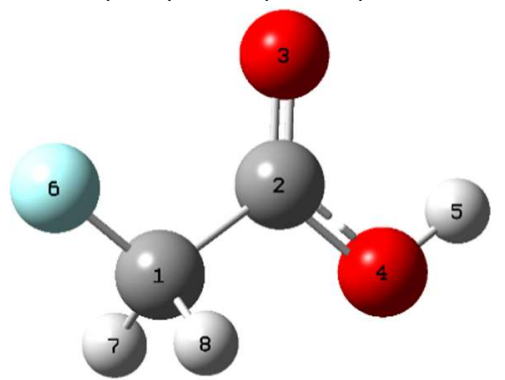

C, $, 0,-2.2593628078,0.4569909343,1.0048495087$ C, $0,-1.9861402793,-0.8810639469,0.3590825238$ $\mathrm{O}, 0,-2.784685687,-1.7541994165,0.1836535131$ $\mathrm{O}, 0,-0.6927161102,-0.956104082,0.0010106899$ $\mathrm{H}, 0,-0.550203182,-1.8230016955,-0.4082476995$ F, $0,-3.5634958941,0.5488208207,1.373097636$ $\mathrm{H}, 0,-1.6143033302,0.5649515688,1.8784778854$ $\mathrm{H}, 0,-2.0133951995,1.2480951271,0.2945013727$

\section{$\mathrm{CjF}-\mathrm{C}(=\mathrm{O}) \mathrm{OH}(\sigma=1)$}

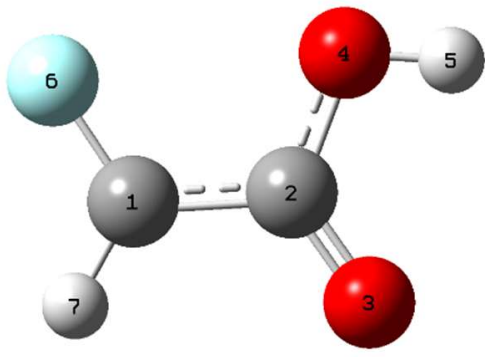

C, $0,0.7761900,0.64608600,0.00032900$ C, $0,-0.55588700,0.10550500,0.00005100$ $\mathrm{O}, 0,-1.53995800,0.81674800,-0.00011900$ $\mathrm{O}, 0,-0.60059600,-1.23737300,-0.00004100$ $\mathrm{H}, 0,-1.5361600,-1.48805300,0.00043200$ F, $0,1.81896500,-0.15130400,-0.00012800$ $\mathrm{H}, 0,0.96809300,1.70523700,-0.00027500$

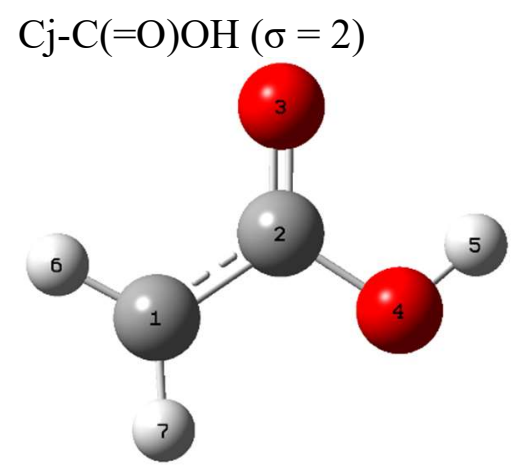

C, $0,1.3069044205,0.1152855668,0.2129023587$ C, $0,-0.1241912543,0.1203740274,0.0394175008$ $\mathrm{O}, 0,-0.7813046032,1.0991941,-0.2502804616$ $\mathrm{O}, 0,-0.6874614634,-1.0928133065,0.2327617731$ $\mathrm{H}, 0,-1.6400135859,-0.9846310369,0.0951179591$ $\mathrm{H}, 0,1.8354137741,1.0420504209,0.0768857937$ $\mathrm{H}, 0,1.8285157022,-0.7901847817,0.4707820762$

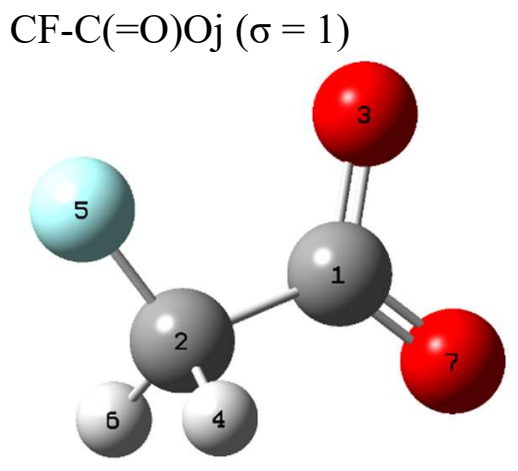

C, $0,-0.0003933182,-0.0000167373,0.0000661406$ C, $0,0.000020429,-0.0000086998,1.5038675357$ $\mathrm{O}, 0,1.0989489216,0.0020297289,-0.6572502949$ H, $0,-0.5282316443,-0.8891037991,1.8499742627$ F, $0,1.2791782252,0.0023212323,1.9740503276$ $\mathrm{H}, 0,-0.5314614588,0.8871638287,1.8499649452$ $\mathrm{O}, 0,-0.9997177544,-0.0018818638,-0.7067279069$

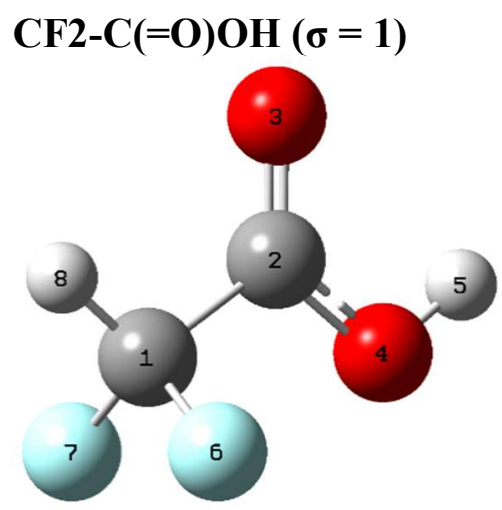

$\mathrm{C}, 0,0.6993544308,0.4440810845,0.0034299405$ $\mathrm{C}, 0,-0.7949276343,0.1180840524,0.0012373089$ $\mathrm{O}, 0,-1.6446441895,0.9642291773,0.0080937954$ $\mathrm{O}, 0,-1.0114585672,-1.1938132283,-0.0090189497$ $\mathrm{H}, 0,-1.9695272077,-1.3443024656,-0.0099778951$ F, $0,1.2748890245,-0.086862997,-1.0921923201$ F, $0,1.2754528173,-0.104064677,1.0902503023$ $\mathrm{H}, 0,0.8446673263,1.5229340538,0.0118968177$

\section{$\mathrm{CjF} 2-\mathrm{C}(=\mathrm{O}) \mathrm{OH}(\sigma=1)$}

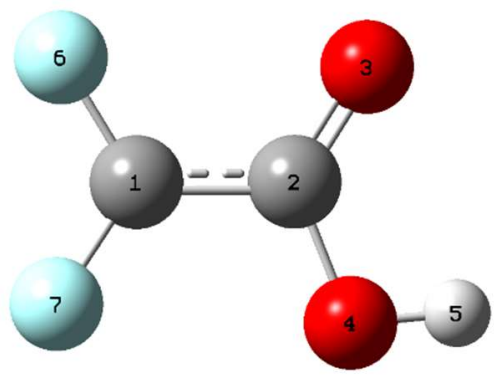

C, $0,0.6653723163,-0.1014732662,-0.0069462324$ $\mathrm{C}, 0,-0.7623915964,0.0289946332,0.039697202$ $\mathrm{O}, 0,-1.4640731785,-0.6296956738,0.7744466397$ $\mathrm{O}, 0,-1.2089997927,0.9603689654,-0.822765926$ $\mathrm{H}, 0,-2.172805372,0.9887392734,-0.7326757637$ F, $0,1.2961924823,-0.9468277233,0.7482849051$ F, $0,1.4386224309,0.5949581812,-0.7876258948$

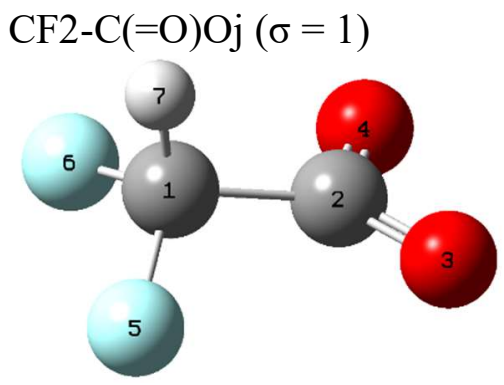

C, $0,0.6983532597,-0.0488546142,-0.3681161072$ C $0,-0.8021874499,-0.0472465786,-0.0531968501$ $\mathrm{O}, 0,-1.5008988158,-1.0292865959,-0.0138363599$ $\mathrm{O}, 0,-1.3492564471,1.1200037373,0.1314794942$ F, $0,1.2592597424,-1.0946204574,0.2496107815$ F, $0,1.2483254074,1.0916602796,0.0869235814$ H, $0,0.8664713033,-0.1282517709,-1.4436475402$

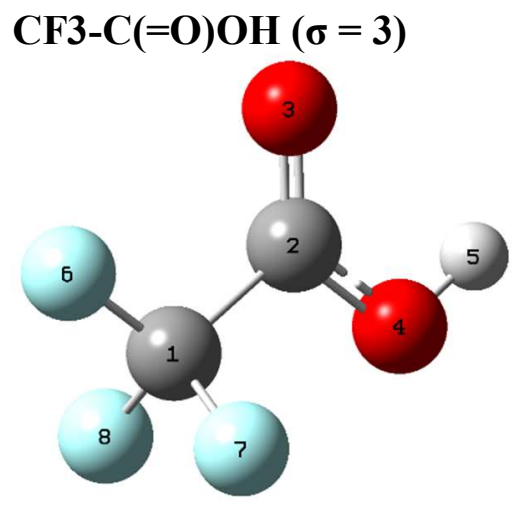

C, $0,-2.2569751345,0.4720893959,0.9995059842$ C, $0,-1.9794949687,-0.8608522163,0.2731113716$ $\mathrm{O}, 0,-2.8367867458,-1.5643197502,-0.1675054833$ $\mathrm{O}, 0,-0.6701534455,-1.0833487043,0.2238687134$ $\mathrm{H}, 0,-0.5167294168,-1.9236841639,-0.2354880104$ $\mathrm{F}, 0,-3.555189807,0.7023210702,1.0529297321$ $\mathrm{F}, 0,-1.6665022102,1.4764048602,0.3563235786$ F, $0,-1.7750817815,0.4276168185,2.2392302837$

\section{$\mathrm{CF} 3-\mathrm{C}(=\mathrm{O}) \mathrm{Oj}(\sigma=3)$}

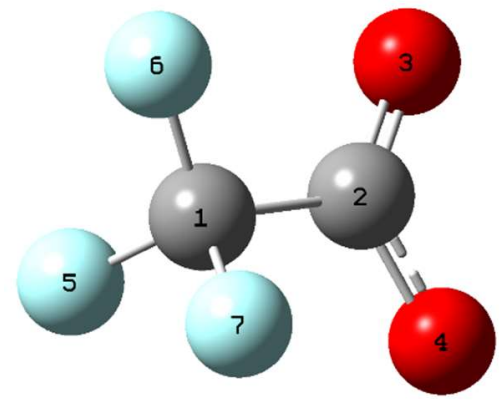

C, $0,-0.5765309439,0.0169865218,0.0043688233$ C, $0,0.9729325285,0.0591605627,0.0128007332$ $\mathrm{O}, 0,1.6279985767,1.0634092084,0.006894323$ $\mathrm{O}, 0,1.5307143579,-1.1236296615,-0.0013189082$ $\mathrm{F}, 0,-1.0044204721,-0.2547849428,-1.2236329336$ F, $0,-1.0489374541,1.1864279302,0.3815474569$ F, $0,-1.0185175929,-0.9286536189,0.8257895054$ 


\section{$\mathrm{CF}-\mathrm{C}-\mathrm{C}(=\mathrm{O}) \mathrm{OH}(\sigma=1)$}

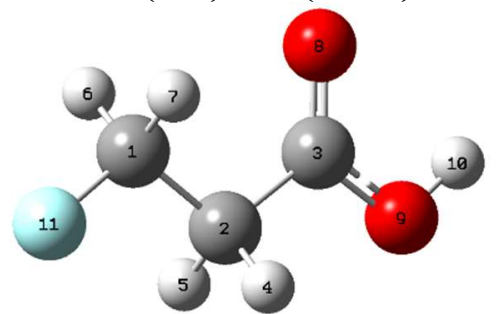

C, $0,-3.0028036934,1.5977519706,0.6703570753$ C, $0,-2.4704906433,0.5227684193,1.5916304455$ C, $0,-1.0046815777,0.3050073755,1.361599248$ $\mathrm{H}, 0,-2.6272804835,0.7945493585,2.6361793971$ $\mathrm{H}, 0,-2.9963474976,-0.4194419225,1.4325997079$ $\mathrm{H}, 0,-2.8677066018,1.3187052428,-0.3739174187$ $\mathrm{H}, 0,-2.4946896355,2.5458385231,0.8423910651$ $\mathrm{O}, 0,-0.3378790675,0.9012301035,0.5545240429$ $\mathrm{O}, 0,-0.4959403998,-0.6435108855,2.1632332981$ $\mathrm{H}, 0,0.4473686284,-0.72306774455,1.953453966$ F, $0,-4.3495974683,1.7650929293,0.9146212628$

\section{$\mathrm{CjF}-\mathrm{C}-\mathrm{C}(=\mathrm{O}) \mathrm{OH}(\sigma=1)$}

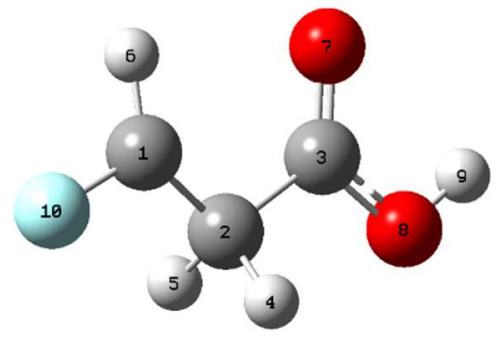

C, $0,1.3875029463,0.2756372683,0.1226605179$ C, $0,0.2470177821,-0.65644116,0.0500178369$ C, $0,-1.0639218706,0.0802347664,0.0484229308$ $\mathrm{H}, 0,0.2934065681,-1.2907754418,-0.8401467951$ $\mathrm{H}, 0,0.2322495376,-1.354025351,0.8986655564$ $\mathrm{H}, 0,1.3279437418,1.2878941278,0.4828830763$ $\mathrm{O}, 0,-1.2037396399,1.2628391167,0.2163742297$ $\mathrm{O}, 0,-2.0971877946,-0.7526687485,-0.1545395802$ $\mathrm{H}, 0,-2.9065854996,-0.2195095647,-0.1293436432$ F, $0,2.5921172289,-0.2966510333,0.1894368706$

\section{$\mathrm{Cj}-\mathrm{C}-\mathrm{C}(=\mathrm{O}) \mathrm{OH}(\sigma=1)$}

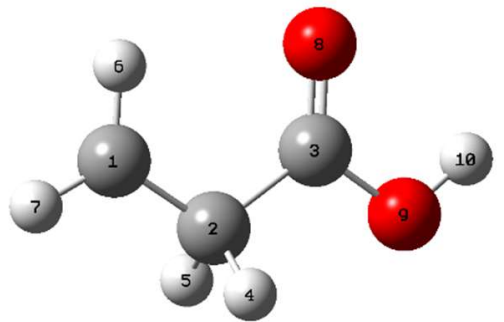

C, $0,1.2392795104,0.6019754467,-0.0143947332$ C, $0,0.2040331845,-0.433035097,-0.2127606814$ C, $0,-1.1758417688,0.0295447202,0.1738950569$ $\mathrm{H}, 0,0.1478226499,-0.7777132477,-1.2525437709$ $\mathrm{H}, 0,0.4084529329,-1.3470824904,0.3580126322$ $\mathrm{H}, 0,0.9624106467,1.5680219702,0.3708959586$ $\mathrm{H}, 0,2.2676266539,0.3929091693,-0.2540132023$ $\mathrm{O}, 0,-1.4660222079,1.1141365433,0.6039539692$ $\mathrm{O}, 0,-2.0907294782,-0.9382240662,-0.0199372856$ $\mathrm{H}, 0,-2.9495791234,-0.5769659584,0.2469220565$
$\mathrm{CF}-\mathrm{Cj}-\mathrm{C}(=\mathrm{O}) \mathrm{OH}(\sigma=1)$

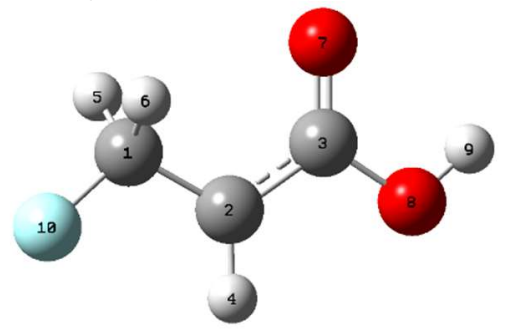

C, $0,-1.39704600,-0.39699700,0.00032500$ C $, 0,-0.2452900,0.52550900,0.0002800$ C, $0,1.0761300,-0.04804800,0.0000200$ $\mathrm{H}, 0,-0.39785500,1.59217800,0.00032400$ $\mathrm{H}, 0,-1.36731800,-1.04981900,0.87902100$ $\mathrm{H}, 0,-1.36667500,-1.0506500,-0.87771500$ $\mathrm{O}, 0,1.28499600,-1.24651900,-0.0001600$ $\mathrm{O}, 0,2.06907300,0.86269900,-0.00007100$ $\mathrm{H}, 0,2.90400700,0.37132900,-0.00014300$ F, $0,-2.57860900,0.30274900,-0.00037600$

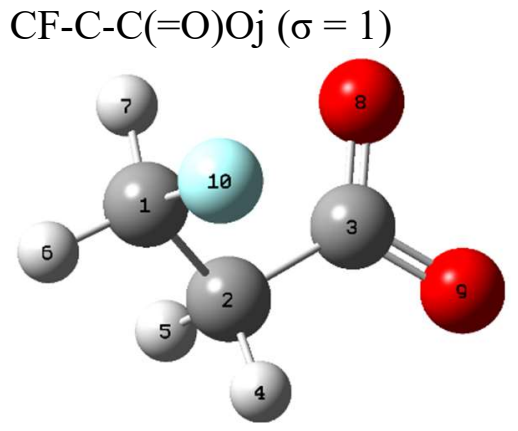

C, $0,-1.4798194024,-0.091357811,-0.4677278058$ C, $0,-0.2038123722,-0.8991147113,-0.370980092$ C $, 0,0.9654966921,-0.017823072,-0.0638172026$ $\mathrm{H}, 0,-0.2865732045,-1.6718274215,0.392325362$ H,0,0.0028015244,-1.3901023591,-1.3244424105 $\mathrm{H}, 0,-2.2990258908,-0.7085869949,-0.8348299754$ $\mathrm{H}, 0,-1.3451732604,0.7679196276,-1.1245958241$ $\mathrm{O}, 0,1.0815542342,1.1626751485,-0.402194182$ $\mathrm{O}, 0,1.9887444989,-0.4383487521,0.5648244778$ F, $0,-1.8077438191,0.3693383457,0.7867896528$

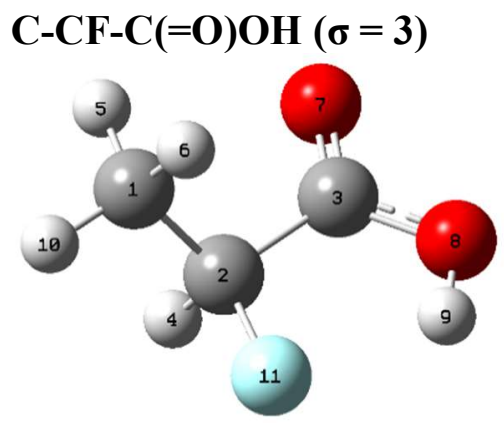

C, $0,-1.5001560531,0.9794586021,0.3900375299$ C, $0,-0.6548954602,0.0200289177,-0.4107695787$ C, $0,0.8236402723,0.1752414542,-0.0745146892$ $\mathrm{H}, 0,-0.7677743832,0.1867704245,-1.4822195636$ $\mathrm{H}, 0,-1.1461354291,1.992328081,0.2117025467$ $\mathrm{H}, 0,-1.4112116124,0.7584699337,1.452724891$ $\mathrm{O}, 0,1.3821704021,1.2276985765,-0.1977071673$ $\mathrm{O}, 0,1.431832382,-0.9299097471,0.3579707257$ $\mathrm{H}, 0,0.7890284355,-1.6558137651,0.3837691925$ $\mathrm{H}, 0,-2.5436187451,0.9007878268,0.0940852665$ F, $0,-1.0493598088,-1.2905063043,-0.1519601535$

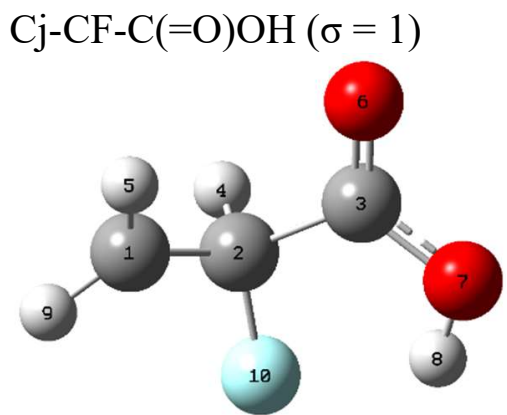

C, $0,1.5007547215,-1.0385908123,0.1426684669$ C, $0,0.6568751856,-0.0093058833,-0.4851503462$ C, $0,-0.812103391,-0.1908032602,-0.0987649908$ $\mathrm{H}, 0,0.6999443628,-0.0450842079,-1.5809483372$ $\mathrm{H}, 0,1.0683172162,-1.991537997,0.3914891434$ $\mathrm{O}, 0,-1.3717763501,-1.2348304298,-0.2673318007$ $\mathrm{O}, 0,-1.3977868771,0.8880064201,0.4153671918$ $\mathrm{H}, 0,-0.7464225508,1.6042303142,0.4796110035$ $\mathrm{H}, 0,2.5568084068,-0.8582842136,0.2463705987$ F, $0,1.081016286,1.2699880698,-0.1222369395$

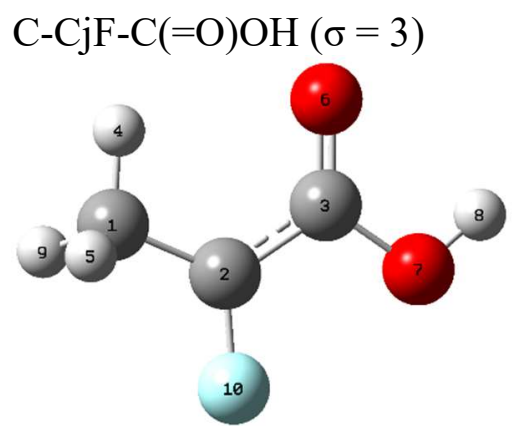

C, $0,1.74620200,-0.83324100,0.00003700$ $\mathrm{C}, 0,0.63685300,0.12942400,-0.00011600$ C, $0,-0.75811300,-0.22684400,0.00000000$ $\mathrm{H}, 0,1.3416600,-1.84113100,-0.00016900$ $\mathrm{H}, 0,2.37813100,-0.69445200,0.88051400$ $\mathrm{O}, 0,-1.14542900,-1.38064200,-0.00001300$ $\mathrm{O}, 0,-1.59039400,0.83073700,0.0000800$ $\mathrm{H}, 0,-2.49022600,0.47311400,-0.00024700$ $\mathrm{H}, 0,2.37856800,-0.6942100,-0.88009300$ $\mathrm{F}, 0,0.94764400,1.41554400,-0.00000700$

\section{$\mathrm{C}-\mathrm{Cj}-\mathrm{C}(=\mathrm{O}) \mathrm{OH}(\sigma=3)$}

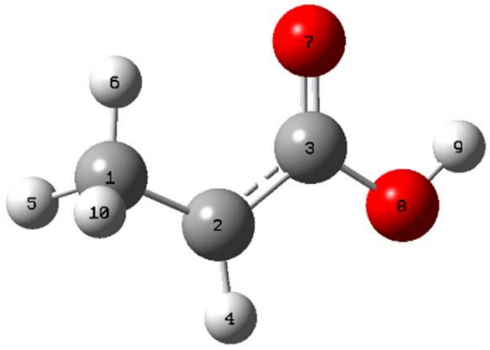

C, $0,-1.9934711725,0.0233962199,0.0005662673$ C, $0,-0.6918453172,-0.6688876858,0.0017407755$ C, $0,0.5419140137,0.0800448522,-0.0007570855$ $\mathrm{H}, 0,-0.6276572273,-1.7461034074,0.0044741455$ H, $0,-2.5884033742,-0.2594330313,-0.8725332295$ $\mathrm{H}, 0,-1.8481437075,1.1010560328,-0.0022618879$ $\mathrm{O}, 0,0.6228986682,1.2932807886,-0.0039178084$ $\mathrm{O}, 0,1.6403272505,-0.7094649136,0.0007709903$ $\mathrm{H}, 0,2.4073136734,-0.1183995492,-0.0011001058$ $\mathrm{H}, 0,-2.5875998071,-0.2549563062,0.8756489383$ 


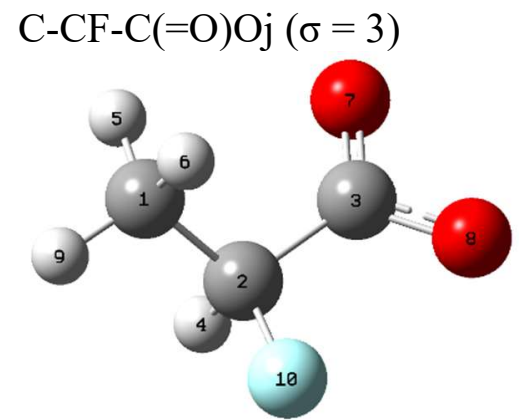

C, $0,1.4990988965,-0.9885131529,0.3952714967$ C, $0,0.6821121924,0.0052163824,-0.3957861472$ C, $0,-0.7901575341,-0.1580287892,-0.0971565134$ $\mathrm{H}, 0,0.8095270513,-0.1459204345,-1.4703383952$ $\mathrm{H}, 0,1.1342584366,-1.9945672497,0.1953507622$ $\mathrm{H}, 0,1.4043876253,-0.7831126716,1.4600263214$ $\mathrm{O}, 0,-1.4083744205,-1.2067394291,-0.1479442389$ $\mathrm{O}, 0,-1.4870521696,0.8780050114,0.2356350658$ $\mathrm{H}, 0,2.5456461117,-0.9210168524,0.1081109836$ F, $0,1.0459998103,1.2949641856,-0.0949133249$

\section{CF2-C-C $(=0) O H(\sigma=1)$}

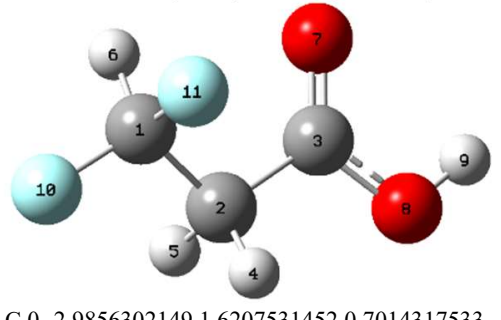

C, $0,-2.9856302149,1.6207531452,0.7014317533$ C, $0,-2.4716831886,0.5068089436,1.5785196959$ C, $0,-1.020418483,0.2309448204,1.2964888887$ $\mathrm{H}, 0,-2.6160557266,0.7706063012,2.6247160519$ H,0,-3.0398222583,-0.4030797573,1.3817251973 $\mathrm{H}, 0,-2.7473676223,1.4848341063,-0.3523235695$ $\mathrm{O}, 0,-0.4396512919,0.5600471809,0.2960571138$ $\mathrm{O}, 0,-0.4396674257,-0.4594721026,2.2873661551$ $\mathrm{H}, 0,0.4809864817,-0.6213830949,2.0286440648$ F, $0,-4.3304948259,1.7163582925,0.8439433691$ F, $0,-2.4495111944,2.7952186446,1.1107117496$

\section{$\mathrm{CjF} 2-\mathrm{C}-\mathrm{C}(=\mathrm{O}) \mathrm{OH}(\sigma=1)$}

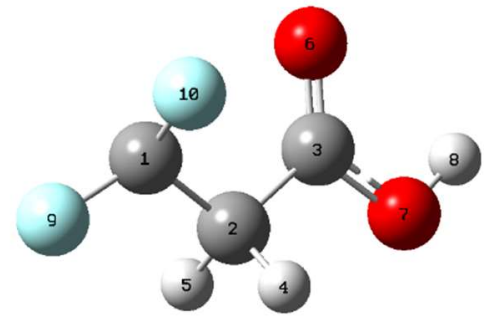

C, $0,1.1933073976,0.0149923759,0.209623975$ C, $0,-0.0320640874,-0.7847936948,-0.0492809258$ C, $0,-1.2831206601,0.0479529926,0.0724620862$ $\mathrm{H}, 0,-0.0021674288,-1.2369949231,-1.0467127631$ $\mathrm{H}, 0,-0.0858894632,-1.6027445809,0.6682626902$ $\mathrm{O}, 0,-1.3362760925,1.1810857325,0.4617786977$ $\mathrm{O}, 0,-2.3624184524,-0.6546191859,-0.3071189031$ $\mathrm{H}, 0,-3.1358201231,-0.080011697,-0.1984314009$ F, $0,2.285718574,-0.7026428347,0.4375001478$ F, $0,1.445395336,0.9704147956,-0.6678975938$

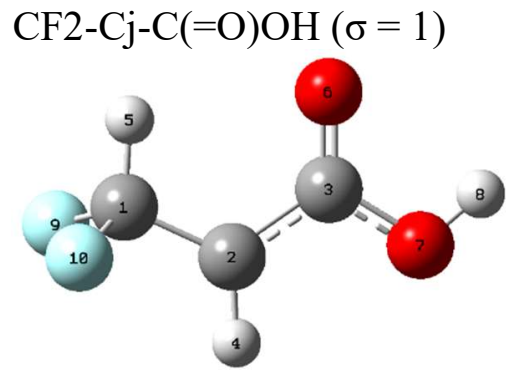

C, $0,-1.1794685913,-0.2282099399,-0.0015855476$ C, $0,0.0619134233,0.5844715743,0.002914738$ C, $0,1.3500720791,-0.0729575342,-0.0006452729$ $\mathrm{H}, 0,-0.0172979954,1.6594465932,0.0089339336$ $\mathrm{H}, 0,-0.9793936323,-1.2978617498,-0.0084721903$ $\mathrm{O}, 0,1.4972642274,-1.2782123753,-0.0074322361$ $\mathrm{O}, 0,2.3813905213,0.7889055828,0.0043208797$ $\mathrm{H}, 0,3.1967147072,0.2651905499,0.0014729173$ F, $0,-1.9228848003,0.0813418602,1.0915186931$ F,0,-1.924038939,0.0952374389,-1.0898499148

\section{CF2-C-C $(=0) O j(\sigma=1)$}

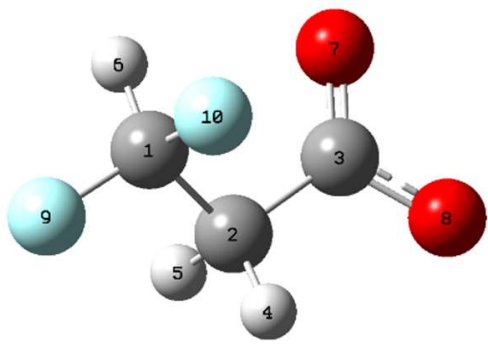

C, $0,1.1787130559,0.1239504794,0.2057032378$ C, $0,-0.0066410139,-0.8041303545,0.0462597572$ C, $0,-1.2843073553,-0.0145870489,0.1293213466$ $\mathrm{H}, 0,0.0758494626,-1.3319419183,-0.9031206129$ $\mathrm{H}, 0,-0.0061706769,-1.5342522975,0.8556731389$ $\mathrm{H}, 0,1.1072828764,0.7637221419,1.0838046024$ $\mathrm{O}, 0,-1.4852874798,0.9495158449,0.8308115412$ $\mathrm{O}, 0,-2.2597008195,-0.454307855,-0.6278375045$ F, $0,2.3080121246,-0.6161645192,0.2834843267$ F, $0,1.2748338261,0.908838527,-0.8917098333$

\section{$\mathrm{CF}-\mathrm{CF}-\mathrm{C}(=\mathrm{O}) \mathrm{OH}(\sigma=1)$}

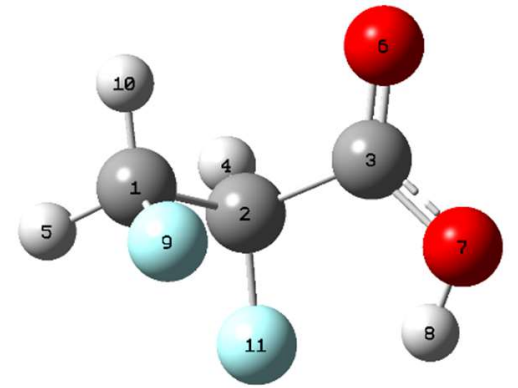

C, $0,1.4209290543,-0.5608533044,-0.3506941523$ C, $0,0.2606266486,0.3244194681,-0.7255169813$ C, $0,-1.0224577031,-0.2276374712,-0.110481173$ $\mathrm{H}, 0,0.1383391816,0.3499376671,-1.8082612861$ H, $0,2.3539988407,-0.1385535292,-0.7222056307$ $\mathrm{O}, 0,-1.4371414001,-1.3021879411,-0.4364319547$ $\mathrm{O}, 0,-1.6134601169,0.5564458374,0.7848565547$ $\mathrm{H}, 0,-1.0881376276,1.3624610092,0.9065872737$ F, $0,1.4861941791,-0.6419234345,1.0191672644$ H, $0,1.2555713393,-1.5553178477,-0.7640743681$ F, $0,0.492375604,1.6118515465,-0.2730795467$

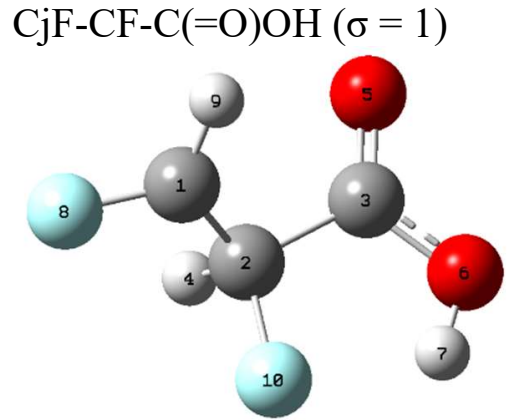

C, $0,1.2825304186,-0.4935958984,0.3597080973$ C, $0,0.2429219356,0.2429900405,-0.3601738824$ C, $0,-1.1247607847,-0.3734557505,-0.0743685009$ $\mathrm{H}, 0,0.4199690012,0.2179442285,-1.4391853021$ $\mathrm{O}, 0,-1.301132826,-1.5547164625,-0.1500200833$ $\mathrm{O}, 0,-2.0768637075,0.4987333621,0.2409545009$ $\mathrm{H}, 0,-1.6930193782,1.389152856,0.2868295707$ F, $0,2.5335523717,-0.2889599573,-0.0204876551$ $\mathrm{H}, 0,1.1148878693,-1.2924321782,1.059871126$

F, $0,0.2187611,1.6025747599,-0.006508871$

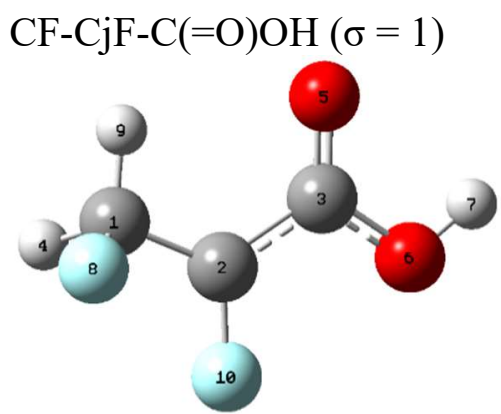

C, $0,-1.4303001686,-0.4629001881,0.4582880153$ C, $0,-0.1946223013,0.2982100018,0.1901961141$ C, $0,1.1045961521,-0.3077117015,-0.0003930967$ $\mathrm{H}, 0,-1.9101554943,-0.0968984989,1.3686493027$ $\mathrm{O}, 0,1.2771206893,-1.5087706166,0.0465348672$ $\mathrm{O}, 0,2.0844583869,0.5801232215,-0.2221789298$ $\mathrm{H}, 0,2.9045686796,0.076696139,-0.3336940519$ F, $0,-2.3235008382,-0.2981025981,-0.5820458001$ $\mathrm{H}, 0,-1.1779961191,-1.5159765397,0.5603396721$ F, $0,-0.2935339865,1.6093367805,0.137996907$

\section{$\mathrm{CF}-\mathrm{CF}-\mathrm{C}(=\mathrm{O}) \mathrm{Oj}(\sigma=1)$}

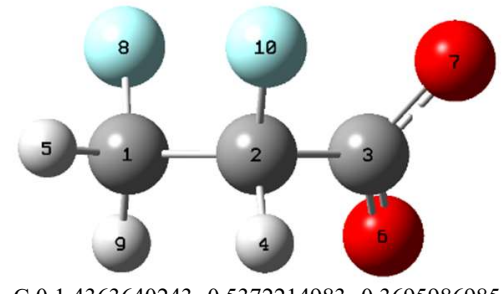

C, $0,1.4363640243,-0.5372214983,-0.3695986985$ C, $0,0.2601927594,0.344877988,-0.7085559423$ C, $0,-0.9964798618,-0.2434937146,-0.095522011$ $\mathrm{H}, 0,0.1188827793,0.3693078697,-1.7917783571$ H, $0,2.3531110208,-0.0928836177,-0.7564221778$ $\mathrm{O}, 0,-1.3963058001,-1.3674756141,-0.3004682359$ O, $0,-1.6938373497,0.5240571102,0.6917698079$ F, $0,1.5324351011,-0.648343421,0.9927877995$ $\mathrm{H}, 0,1.2779220121,-1.5254704251,-0.8026518011$ F, $0,0.4522553145,1.615886313,-0.2433593734$ 
$\mathrm{C}-\mathrm{CF} 2-\mathrm{C}(=\mathrm{O}) \mathrm{OH}(\sigma=3)$

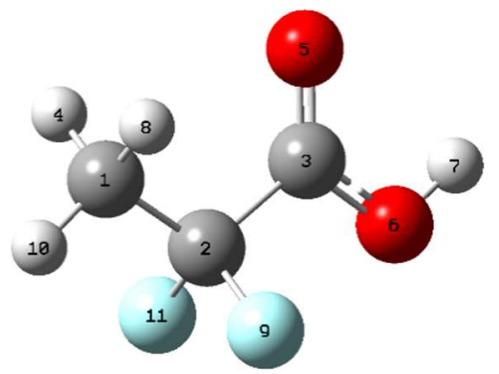

C, $0,-3.0333832497,1.6047781884,0.6726622181$ C, $0,-2.4718100831,0.5445698548,1.5698328862$ C, $0,-0.9684260215,0.302559201,1.3529020568$ $\mathrm{H}, 0,-2.8901492568,1.3170378707,-0.365305915$ $\mathrm{O}, 0,-0.3095731294,0.9004727899,0.5477646942$ $\mathrm{O}, 0,-0.5071064246,-0.6435188117,2.165699437$ $\mathrm{H}, 0,0.4396941418,-0.7570387995,1.9898523292$ $\mathrm{H}, 0,-2.5170995777,2.5440673735,0.8511988546$ F, $0,-2.6502450175,0.8766124458,2.8725753067$ $\mathrm{H}, 0,-4.0933603699,1.710818785,0.8907537997$ F, $0,-3.1100596717,-0.6357652377,1.3731797924$

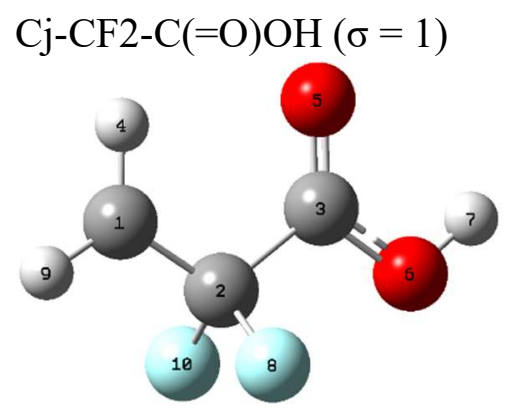

C, $0,1.5031554299,0.9873655694,0.1999018736$ C, $0,0.5892438702,-0.1575965518,0.0725628527$ C, $0,-0.8936031834,0.2517168924,0.0624477413$ $\mathrm{H}, 0,1.1092164646,1.9839578044,0.2741742242$ $\mathrm{O}, 0,-1.2770648372,1.3853673664,0.1489480086$ $\mathrm{O}, 0,-1.6798501094,-0.8132115343,-0.0540605161$ $\mathrm{H}, 0,-2.6008607191,-0.510116715,-0.0561127331$ F, $0,0.8354152188,-0.8553714738,-1.0708689707$ $\mathrm{H}, 0,2.5602220931,0.7860136078,0.2149565102$ F, $0,0.7689057825,-1.0428249756,1.0918880293$

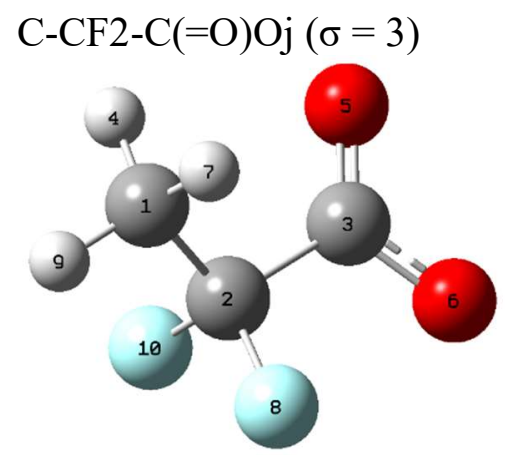

C, $0,1.388667333,0.8008221499,-0.9099009078$ C, $0,0.5794026972,-0.1662795041,-0.1016601782$ C, $0,-0.9170433689,0.2246993609,-0.06534834$ $\mathrm{H}, 0,1.232484628,1.8056952513,-0.5270999184$ $\mathrm{O}, 0,-1.3186511309,1.3594834624,-0.0997173983$ $\mathrm{O}, 0,-1.7356534269,-0.7899395268,0.0325319291$ $\mathrm{H}, 0,1.0798826679,0.7599916399,-1.9515830025$ F, $0,0.6903675645,-1.4277434583,-0.5755728674$ $\mathrm{H}, 0,2.4362768133,0.521168221,-0.8216873079$ F, $0,0.9993068328,-0.1849536162,1.1856111112$
$\mathrm{CF3-C}-\mathrm{C}(=\mathrm{O}) \mathrm{OH}(\sigma=3)$

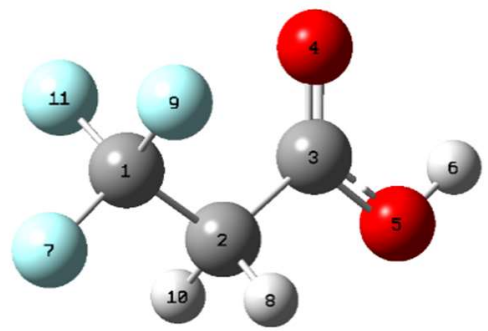

C, $0,-3.0160646918,1.59821606,0.6742779743$ C, $0,-2.4375434382,0.5321093247,1.5717349429$ C, $0,-0.964312192,0.3042810039,1.349684216$ $\mathrm{O}, 0,-0.2694072142,0.875864529,0.558304083$ $\mathrm{O}, 0,-0.5133852426,-0.6520471069,2.1776196438$ $\mathrm{H}, 0,0.4334620783,-0.7664279833,2.0023869781$ $\mathrm{F}, 0,-4.3244752381,1.7382875505,0.9350085263$ $\mathrm{H}, 0,-2.6061059303,0.8202099141,2.6081495005$ F, $0,-2.4420630012,2.7835574068,0.8708009278$ $\mathrm{H}, 0,-2.976521786,-0.398179344,1.3994569538$ F, $0,-2.8984556939,1.2854993651,-0.6146847665$

$$
\mathrm{CF} 3-\mathrm{Cj}-\mathrm{C}(=\mathrm{O}) \mathrm{OH}(\sigma=3)
$$

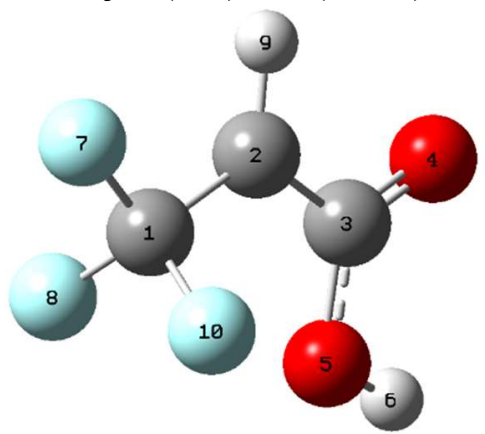

C, $0,0.9220455637,0.2886804439,0.2148824309$ C, $0,-0.2101122303,0.5385994533,-0.7244649128$ C, $0,-1.5668378258,0.0885702565,-0.4750198697$ $\mathrm{O}, 0,-2.4788587344,0.2968398999,-1.2435065836$ $\mathrm{O}, 0,-1.7095598233,-0.5755248379,0.6818249697$ $\mathrm{H}, 0,-2.639877795,-0.83456232,0.7603477354$ F, $0,2.0488052795,0.8152566114,-0.2728784326$ $\mathrm{F}, 0,0.7029360494,0.8379264276,1.4119127037$ $\mathrm{H}, 0,-0.0143437091,1.0792045644,-1.6336735349$ F, $0,1.1321852154,-1.016533499,0.4014825037$

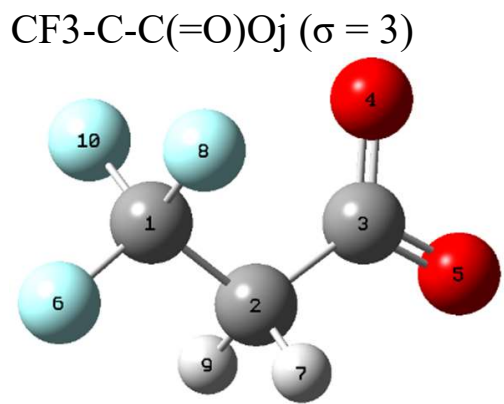

C, $0,1.0616878791,0.067213184,-0.0000907561$ C, $0,-0.2098937786,0.8854019472,-0.0010356173$ C, $0,-1.4555322497,0.0547588121,-0.0001849721$ $\mathrm{O}, 0,-1.5005602734,-1.1921159766,0.0014085934$ $\mathrm{O}, 0,-2.6019014823,0.5560701077,-0.0010169514$ F, $0,2.1231162289,0.8785510738,-0.0009908557$ $\mathrm{H}, 0,-0.2176075437,1.5261337479,0.8795990662$ F, $0,1.1436597852,-0.7088848908,1.079574882$ $\mathrm{H}, 0,-0.2175301313,1.5242216313,-0.8830587629$ F, $0,1.1436875658,-0.7113176365,-1.0780006261$
$\mathrm{CF} 2-\mathrm{CF}-\mathrm{C}(=\mathrm{O}) \mathrm{OH}(\sigma=1)$

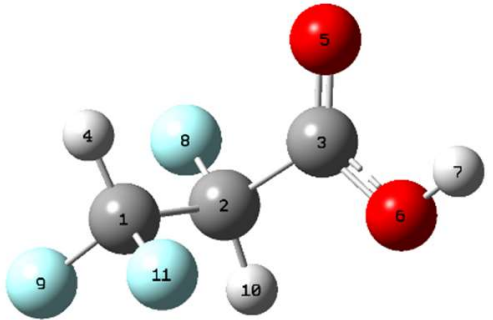

C, $0,-1.1246882114,-0.2281915047,0.3693847197$ C, $, 0,-0.0583653902,0.4757569822,-0.4589207785$ C $0,1.3140297395,0.0257273259,0.0174935379$ $\mathrm{H}, 0,-1.0749804445,0.0729148112,1.4168093908$ $\mathrm{O}, 0,1.8606491594,0.4784825341,0.9824316422$ $\mathrm{O}, 0,1.8028390694,-0.9499079746,-0.7517523789$ $\mathrm{H}, 0,2.6439681901,-1.2442638585,-0.3682287155$ F, $0,-0.1939973687,1.8134212082,-0.2448329502$ F, $0,-2.3388419117,0.0755340453,-0.1241639442$ H, $0,-0.2061629523,0.2422630255,-1.5129545624$ F, $0,-0.9468338795,-1.563489595,0.269536039$

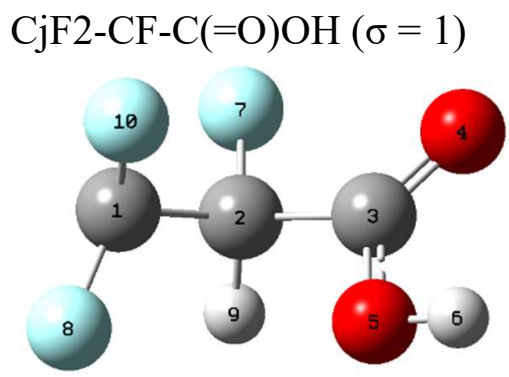

C, $0,-1.1960311962,-0.2352374928,0.0268007391$ C, $0,-0.0758940479,0.6841965621,-0.3542182885$ C, $0,1.2785676362,0.0382966343,-0.0650128908$ $\mathrm{O}, 0,2.1129366315,0.4804309988,0.6645188738$ $\mathrm{O}, 0,1.3766004076,-1.1090088906,-0.7493366625$ $\mathrm{H}, 0,2.22743632,-1.5215258248,-0.5351916388$ F,0,-0.1657856117,1.8467516004,0.3417980298 F, $0,-1.6133299631,-1.0539248065,-0.9169752189$ $\mathrm{H}, 0,-0.155011348,0.8738899862,-1.4258630292$ F, $0,-1.0627018184,-0.8627107572,1.1793280959$

\section{$\mathrm{CF} 2-\mathrm{CjF}-\mathrm{C}(=\mathrm{O}) \mathrm{OH}(\sigma=1)$}

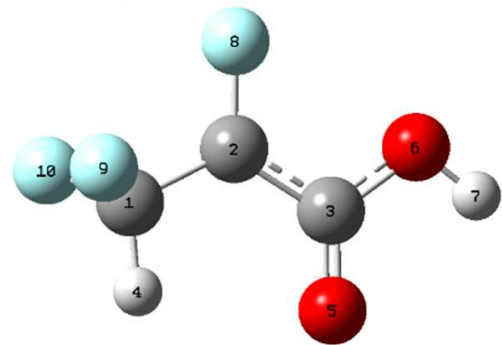

C, $0,-1.2183543555,-0.1571112749,0.5629896084$ C, $0,-0.0350123148,-0.0092174417,-0.327990525$ C, $0,1.3245737692,-0.1391832048,0.1436104073$ $\mathrm{H}, 0,-0.9099314417,-0.3695316388,1.5844487356$ $\mathrm{O}, 0,1.5809870482,-0.3794574967,1.3056870132$ $\mathrm{O}, 0,2.2430672665,0.0298157272,-0.8140315394$ $\mathrm{H}, 0,3.1141008919,-0.0761015065,-0.4028694584$ F, $0,-0.2752158436,0.2498130082,-1.5879273498$ F, $0,-1.9541421404,0.9785204178,0.529988333$ F, $0,-2.0087798897,-1.1551915997,0.1027217952$ 


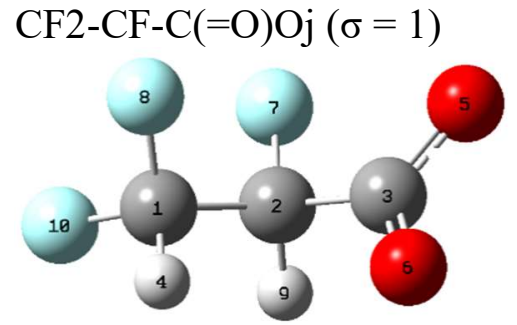

C, $0,-1.0861773733,-0.5383470954,-0.0588398225$ C, $0,0.0179911243,0.3735731364,-0.5771700301$ C, $0,1.3549403045,-0.2071698047,-0.1582375894$ $\mathrm{H}, 0,-0.9331217195,-1.5678351622,-0.3843237844$ $\mathrm{O}, 0,2.1543107546,0.5859795673,0.5033687411$ $\mathrm{O}, 0,1.7115833383,-1.335519332,-0.402907919$ F, $0,-0.1419328614,1.6296424361,-0.0841612738$ F, $0,-1.0930644067,-0.4901367951,1.2875519129$ $\mathrm{H}, 0,-0.0191815584,0.3907963513,-1.6680870376$ F, $0,-2.2730846025,-0.0871133017,-0.5044431973$

\section{CF-CF2-C $(=0)$ OH $(\sigma=1)$}

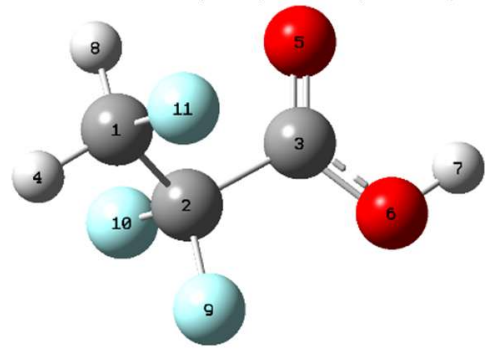

C, $0,1.4773506572,0.3680476263,0.3642340879$ C, $0,0.2704426605,-0.5072190012,0.1035509486$ C, $0,-1.0250367608,0.3212460706,0.1178770625$ $\mathrm{H}, 0,2.3700336461,-0.2557977031,0.3369477317$ $\mathrm{O}, 0,-1.2187990021,1.1515422402,0.9607938468$ $\mathrm{O}, 0,-1.8665028834,-0.002922914,-0.8553481561$ $\mathrm{H}, 0,-2.6585692793,0.5495811398,-0.7612459819$ $\mathrm{H}, 0,1.3603638894,0.8492893222,1.3331531325$ F, $0,0.4096395307,-1.1694464298,-1.0548361664$ F, $0,0.1864796418,-1.4182319887,1.1021722619$ F, $0,1.5383628999,1.3149616378,-0.6219427673$

\section{$\mathrm{CjF}-\mathrm{CF} 2-\mathrm{C}(=\mathrm{O}) \mathrm{OH}(\sigma=1)$}

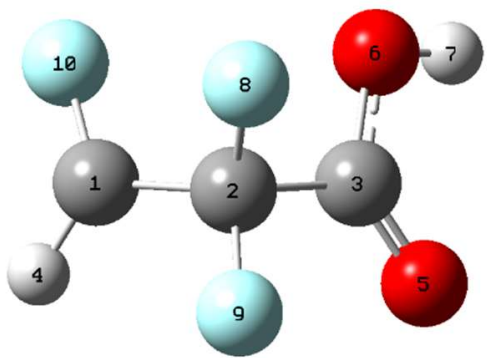

C, $0,1.3473238022,-0.0797083065,0.7061634876$ C, $0,0.2136259642,-0.5394891873,-0.1361368589$ C, $0,-1.0660384775,0.2909462373,0.0939645374$ $\mathrm{H}, 0,1.5700538504,-0.5066036948,1.6692702306$ $\mathrm{O}, 0,-1.9882857787,-0.08402954,0.7565279381$ $\mathrm{O}, 0,-0.9613585544,1.4836173444,-0.4901728622$ $\mathrm{H}, 0,-1.760437745,1.9930202011,-0.2834917949$ F, $0,0.5401407905,-0.4705511108,-1.4475819335$ F, $0,-0.0500538834,-1.8146679177,0.1864527528$ $\mathrm{F}, 0,1.8349180316,1.1153169742,0.4414625031$

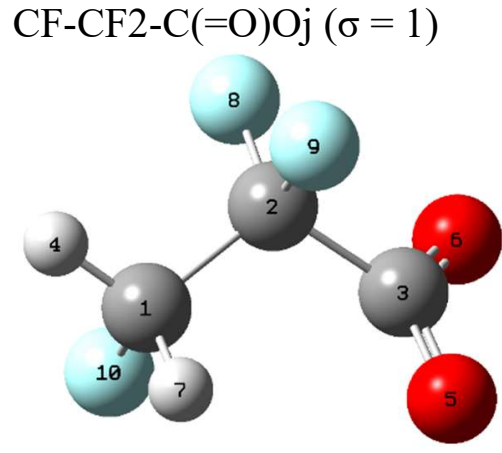

C, $0,-1.4778676308,-0.3730175072,0.3477457856$ C, $0,-0.2821743536,0.5264050438,0.1200457672$ C, $0,1.019042165,-0.3089800476,0.1282128537$ $\mathrm{H}, 0,-2.3794021461,0.2387861669,0.320563885$ $\mathrm{O}, 0,1.3006642108,-1.0613948024,1.0221684537$ $\mathrm{O}, 0,1.7857829051,-0.1265671204,-0.9144226597$ $\mathrm{H}, 0,-1.3660123227,-0.8672043133,1.3115949603$ F, $0,-0.3951723958,1.1995402295,-1.0374947027$ F, $0,-0.2104992034,1.4156260023,1.1280963076$ F, $0,-1.5064582286,-1.3006746515,-0.6548546506$

\section{CF3-CF-C $(=0) \mathrm{OH}(\sigma=3)$}

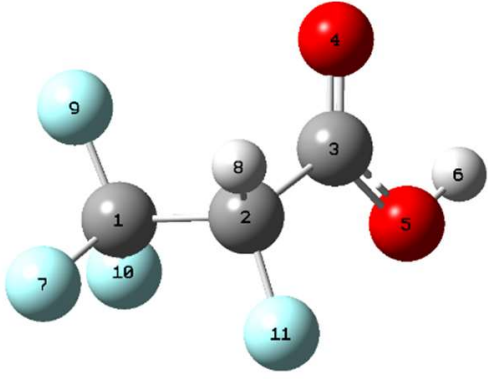

C, $0,-2.9839162769,1.4292480847,0.5794894062$ C, $0,-2.4524200089,0.6708490648,1.7983673892$ C, $0,-0.9573397625,0.4261804612,1.6393166868$ $\mathrm{O}, 0,-0.1455446651,1.2876676145,1.8299481261$ $\mathrm{O}, 0,-0.684506971,-0.817863921,1.2588347467$ H, $0,0.2778592334,-0.8937712228,1.161642526$ F, $0,-4.2990888845,1.5878884161,0.6828007251$ $\mathrm{H}, 0,-2.6073431748,1.2956147022,2.6775144059$ F, $0,-2.4169983014,2.6267616945,0.4919032398$ F, $0,-2.7314707967,0.7569064639,-0.5415844272$ F, $0,-3.1548354418,-0.4848237581,1.9038977754$

\section{$\mathrm{CF} 3-\mathrm{CjF}-\mathrm{C}(=\mathrm{O}) \mathrm{OH}(\sigma=3)$}

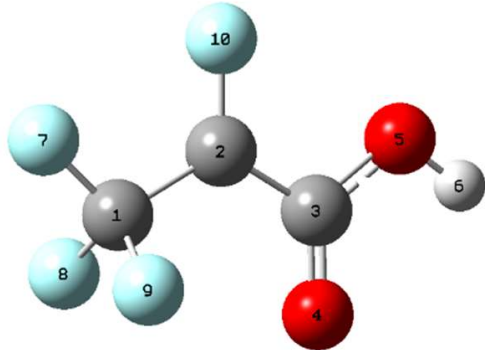

C, $0,-1.1493491999,-0.1694529594,-0.0407111868$ C, $0,0.1979630719,0.5055107913,-0.0625485327$ C, $0,1.4178973083,-0.2054670004,0.2575716187$ $\mathrm{O}, 0,1.4144369474,-1.3768605913,0.5581768145$ $\mathrm{O}, 0,2.5099075703,0.5649087475,0.182620905$ $\mathrm{H}, 0,3.2721005799,0.0113307552,0.4105455971$ $\mathrm{F}, 0,-2.0914353331,0.7073320651,-0.3721868201$ F, $0,-1.1951943822,-1.182252376,-0.902952963$

F $0,-1.4285373423,-0.649870283,1.1686562832$ F, $0,0.2226837798,1.7708408511,-0.3847747159$
$\mathrm{CF} 3-\mathrm{CF}-\mathrm{C}(=\mathrm{O}) \mathrm{Oj}(\sigma=3)$

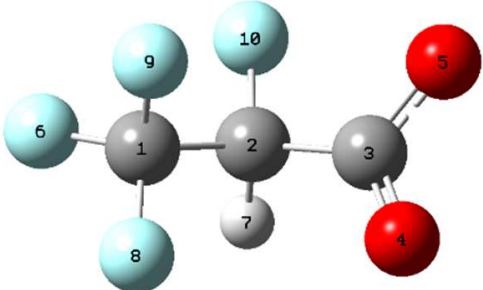

C, $0,-1.0762683851,-0.2043316552,0.0616326166$ C, $0,0.1223958723,0.4214519665,-0.6544028745$ C, $0,1.4162679652,-0.1914776923,-0.1524722772$ $\mathrm{O}, 0,1.750891614,-1.3530312412,-0.2788502197$ $\mathrm{O}, 0,2.2602114462,0.5663376117,0.4551159099$ F, $0,-2.1928441311,0.4109599882,-0.3068905503$ $\mathrm{H}, 0,0.0219306885,0.2268177637,-1.7228343345$ F, $0,-1.1705738032,-1.4904710735,-0.2519197544$ F, $0,-0.9475332525,-0.0906159687,1.3796327855$ F, $0,0.1278859858,1.754961311,-0.4057492914$

\section{CF2-CF2-C $(=0) \mathrm{OH}(\sigma=1)$}

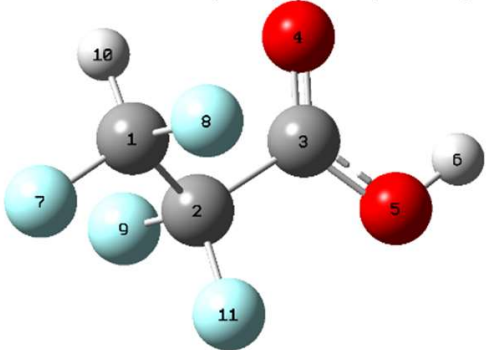

C, $0,-3.2123387545,1.4941328216,0.9298337287$ C, $0,-2.3560149336,0.5309438914,1.7570550672$ C, $0,-1.1832577485,0.0145009416,0.911746749$ $\mathrm{O}, 0,-1.3248617112,-0.2326865206,-0.253389473$ $\mathrm{O}, 0,-0.0696010316,-0.1419126459,1.6115907759$ $\mathrm{H}, 0,0.6138657871,-0.4964763221,1.0207200088$ F, $0,-4.2253067331,1.9256890106,1.6951228447$ F, $0,-2.4397783452,2.5459002552,0.5960917687$ F,0,-3.1195288523,-0.5273959003,2.0987811323 $\mathrm{H}, 0,-3.5931834633,1.0116104566,0.0313225054$ F, $0,-1.9413707338,1.137957382,2.8714141222$

\section{$\mathrm{CjF} 2-\mathrm{CF} 2-\mathrm{C}(=\mathrm{O}) \mathrm{OH}(\sigma=1)$}

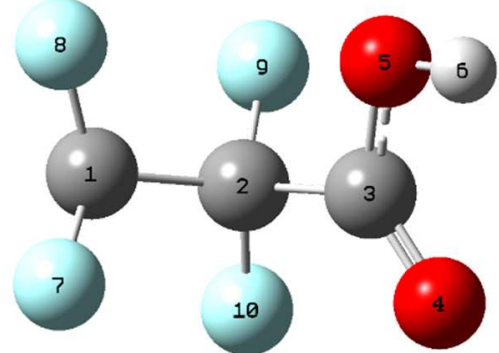

C, $0,1.3137102441,-0.0781749585,-0.1437851016$ C, $0,-0.0282399291,-0.5016570855,0.4112771318$ C, $0,-1.1988016566,0.2837742889,-0.2207741932$ $\mathrm{O}, 0,-1.9676062722,-0.1881422701,-1.0021696693$ $\mathrm{O}, 0,-1.1773597291,1.5479678625,0.1975592618$ $\mathrm{H}, 0,-1.8997438642,2.0284151997,-0.236365181$ F, $0,1.4303090801,-0.2342431318,-1.4435779949$ $\mathrm{F}, 0,1.7427103821,1.0947237487,0.2534395621$ $\mathrm{F}, 0,-0.0166026313,-0.2998434806,1.7388467726$ F, $0,-0.2024566237,-1.7991871733,0.1507784117$ 
CF2-CF2-C $(=\mathrm{O}) \mathrm{Oj}(\sigma=1)$

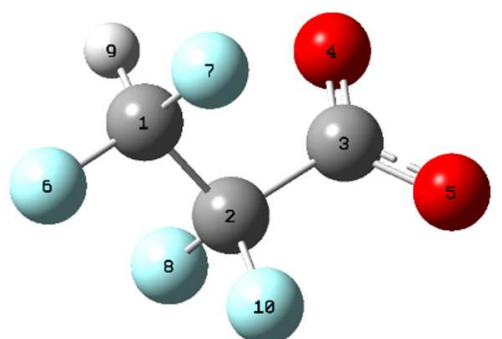

C, $0,-1.1615978343,0.5187194237,-0.236965541$ C, $0,-0.0189232728,-0.49493859,-0.0923392503$ C, $0,1.3273259665,0.268656513,-0.1637219502$ $\mathrm{O}, 0,1.5728376125,1.0317120951,-1.0576314763$ $\mathrm{O}, 0,2.13117517,-0.0025378596,0.8302597444$ $\mathrm{F}, 0,-2.3225507859,-0.1399791165,-0.137128441$ F, $0,-1.0599179444,1.3906992857,0.7828915268$ F, $0,-0.0673205502,-1.3516794936,-1.121310758$ $\mathrm{H}, 0,-1.0993769864,1.0444495568,-1.1889050392$ $\mathrm{F}, 0,-0.145695375,-1.1741028145,1.0513641848$

\section{CF3-CF2-C $(=0) O H(\sigma=3)$}

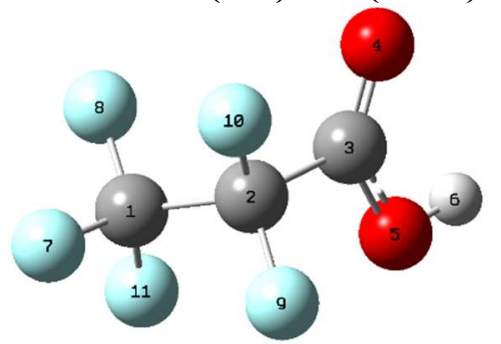

C, $0,1.0684070191,0.2789838201,-0.2122779842$ C, $0,-0.1423319616,-0.505171674,0.3410222139$ C, $0,-1.4807390017,0.0698970187,-0.1658076428$ $\mathrm{O}, 0,-2.0790783911,-0.4017525939,-1.0844308159$ $\mathrm{O}, 0,-1.8334482743,1.1385211745,0.5410912148$ $\mathrm{H}, 0,-2.6646917263,1.4849669097,0.1799792884$ F, $0,2.1919504799,-0.2994106118,0.1791188537$ $\mathrm{F}, 0,1.0210832567,0.2897373431,-1.5386613412$ F, $0,-0.0826075415,-0.4638640707,1.6787296415$ F, $0,-0.0295481263,-1.768192782,-0.0701663742$ F, $0,1.047297267,1.5288384664,0.229985946$

\section{$\mathrm{CF} 3-\mathrm{CF} 2-\mathrm{C}(=\mathrm{O}) \mathrm{Oj}(\sigma=3)$}

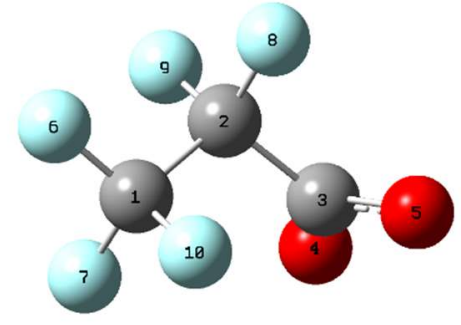

C, $0,-1.0774566159,-0.2665703656,-0.2301565466$ $\mathrm{C}, 0,0.1239384715,0.512608671,0.3481500372$ C, $0,1.4641298184,-0.0808997994,-0.141747388$ $\mathrm{O}, 0,2.0206295478,0.249408074,-1.1518221921$ $\mathrm{O}, 0,1.9509724174,-1.0144695556,0.6331094222$ F, $0,-2.2055450426,0.2509918495,0.2229452089$ F, $0,-1.0607622513,-0.2018286932,-1.5514936988$ F, $0,0.0570712732,0.4471163055,1.6843316429$ F, $0,0.0440678561,1.7778153835,-0.0523436439$ F, $0,-0.9938224745,-1.5370558698,0.1465701584$ 


\section{Vibrational Frequencies}

In this article, explicit hydrogens are not included in species but are implied unless otherwise stated.And $\mathrm{j}$ represents a radical site on the preceding carbon or oxygen atom.

From MN15/cc-pVTZ level of theory

Table S1: Vibrational Frequencies

\begin{tabular}{|c|c|c|c|c|c|c|c|c|c|c|}
\hline \multirow{2}{*}{$\begin{array}{r}\text { Species } \\
\text { CF-C }(=\mathbf{O}) \mathrm{OH}\end{array}$} & \multicolumn{10}{|c|}{ Frequencies $\left(\mathrm{cm}^{-1}\right)$} \\
\hline & 100.04 & 249.88 & 456.94 & 494.53 & 637.08 & 642.49 & 878.70 & 1031.26 & 1153.26 & 1187.83 \\
\hline \multirow{3}{*}{$\mathrm{CjF}-\mathrm{C}(=\mathrm{O}) \mathrm{OH}$} & 1261.79 & 1316.54 & 1446.44 & 1471.44 & 1902.40 & 3090.30 & 3136.44 & 3817.70 & & \\
\hline & 183.05 & 243.16 & 508.04 & 510.81 & 550.70 & 611.30 & 727.14 & 936.61 & 1168.10 & 1241.59 \\
\hline & 1350.79 & 1517.68 & 1768.09 & 3292.41 & 3828.88 & & & & & \\
\hline \multirow[t]{2}{*}{$\mathrm{Cj}-\mathrm{C}(=\mathrm{O}) \mathrm{OH}$} & 324.01 & 407.78 & 540.51 & 574.65 & 646.71 & 782.38 & 936.09 & 980.84 & 1192.57 & 1367.02 \\
\hline & 1465.12 & 1757.86 & 3223.98 & 3345.24 & 3825.98 & & & & & \\
\hline \multirow[t]{2}{*}{$\mathrm{CF}-\mathrm{C}(=\mathrm{O}) \mathrm{Oj}$} & 86.46 & 174.05 & 328.97 & 506.53 & 509.22 & 808.76 & 1013.25 & 1153.19 & 1170.15 & 1251.37 \\
\hline & 1377.54 & 1464.71 & 1644.38 & 3099.77 & 3150.25 & & & & & \\
\hline \multirow[t]{2}{*}{$\mathrm{CF} 2-\mathrm{C}(=\mathrm{O}) \mathrm{OH}$} & 34.17 & 232.66 & 251.49 & 411.05 & 573.40 & 582.17 & 665.34 & 791.49 & 914.07 & 1164.97 \\
\hline & 1173.92 & 1186.74 & 1359.66 & 1379.35 & 1444.92 & 1889.88 & 3162.48 & 3810.74 & & \\
\hline \multirow[t]{2}{*}{$\mathrm{CjF} 2-\mathrm{C}(=\mathrm{O}) \mathrm{OH}$} & 124.04 & 172.45 & 222.69 & 408.48 & 493.62 & 524.63 & 593.40 & 685.94 & 798.29 & 1142.57 \\
\hline & 1292.90 & 1445.26 & 1581.26 & 1787.65 & 3832.34 & & & & & \\
\hline \multirow[t]{2}{*}{$\mathrm{CF} 2-\mathrm{C}(=\mathrm{O}) \mathrm{Oj}$} & 34.97 & 169.83 & 226.63 & 339.04 & 479.30 & 531.15 & 664.77 & 907.97 & 1173.46 & 1206.39 \\
\hline & 1276.98 & 1363.08 & 1391.57 & 1688.90 & 3124.04 & & & & & \\
\hline \multirow[t]{2}{*}{$\mathrm{CF3}-\mathrm{C}(=\mathrm{O}) \mathrm{OH}$} & 40.07 & 231.42 & 237.66 & 387.90 & 424.22 & 505.19 & 590.11 & 590.95 & 664.96 & 794.65 \\
\hline & 817.86 & 1165.86 & 1227.66 & 1244.88 & 1307.19 & 1452.12 & 1915.05 & 3811.68 & & \\
\hline \multirow[t]{2}{*}{$\mathrm{CF} 3-\mathrm{C}(=\mathrm{O}) \mathrm{Oj}$} & 15.92 & 186.29 & 226.39 & 252.28 & 417.17 & 493.32 & 513.81 & 598.96 & 743.48 & 802.58 \\
\hline & 1204.13 & 1251.82 & 1282.73 & 1341.34 & 1744.19 & & & & & \\
\hline \multirow[t]{3}{*}{$\mathrm{CF}-\mathrm{C}-\mathrm{C}(=\mathrm{O}) \mathrm{OH}$} & 67.71 & 105.93 & 178.30 & 366.25 & 446.79 & 521.77 & 632.07 & 658.36 & 794.68 & 909.42 \\
\hline & 1082.42 & 1089.57 & 1101.90 & 1178.01 & 1214.67 & 1269.56 & 1293.57 & 1394.46 & 1447.78 & 1453.77 \\
\hline & 1507.33 & 1856.33 & 3094.98 & 3106.47 & 3129.94 & 3158.82 & 3810.87 & & & \\
\hline \multirow[t]{3}{*}{$\mathrm{CjF}-\mathrm{C}-\mathrm{C}(=\mathrm{O}) \mathrm{OH}$} & 65.71 & 85.64 & 187.09 & 341.64 & 398.76 & 454.84 & 515.98 & 633.28 & 658.23 & 907.00 \\
\hline & 928.33 & 1113.99 & 1158.52 & 1162.27 & 1219.85 & 1253.70 & 1378.17 & 1434.24 & 1455.17 & 1865.35 \\
\hline & 3009.10 & 3068.70 & 3282.24 & 3812.59 & & & & & & \\
\hline \multirow[t]{3}{*}{$\mathrm{Cj}-\mathrm{C}-\mathrm{C}(=\mathrm{O}) \mathrm{OH}$} & 71.63 & 157.97 & 271.55 & 460.56 & 472.09 & 527.10 & 605.95 & 649.54 & 819.41 & 905.92 \\
\hline & 998.96 & 1122.07 & 1172.40 & 1175.13 & 1291.57 & 1409.34 & 1426.08 & 1444.26 & 1868.33 & 3025.45 \\
\hline & 3038.26 & 3213.42 & 3323.33 & 3814.96 & & & & & & \\
\hline \multirow[t]{3}{*}{$\mathrm{CF}-\mathrm{Cj}-\mathrm{C}(=\mathrm{O}) \mathrm{OH}$} & 44.00 & 148.07 & 168.42 & 371.89 & 456.74 & 478.15 & 570.86 & 635.86 & 738.78 & 950.49 \\
\hline & 982.12 & 1094.22 & 1102.72 & 1170.34 & 1224.72 & 1263.18 & 1392.24 & 1453.82 & 1494.70 & 1760.53 \\
\hline & 3044.12 & 3073.36 & 3268.74 & 3823.44 & & & & & & \\
\hline \multirow[t]{3}{*}{$\mathrm{CF}-\mathrm{C}-\mathrm{C}(=\mathrm{O}) \mathrm{Oj}$} & 43.46 & 97.37 & 213.42 & 278.86 & 400.81 & 517.39 & 584.52 & 751.12 & 881.42 & 980.41 \\
\hline & 1039.12 & 1076.11 & 1123.69 & 1216.56 & 1269.77 & 1349.23 & 1414.22 & 1423.92 & 1497.40 & 1629.88 \\
\hline & 3091.03 & 3100.95 & 3143.31 & 3154.24 & & & & & & \\
\hline \multirow[t]{3}{*}{$\mathrm{C}-\mathrm{CF}-\mathrm{C}(=\mathrm{O}) \mathrm{OH}$} & 64.23 & 224.64 & 254.50 & 324.33 & 398.53 & 511.51 & 583.49 & 625.91 & 735.60 & 827.60 \\
\hline & 919.44 & 1060.60 & 1129.38 & 1134.36 & 1217.76 & 1317.56 & 1341.75 & 1393.53 & 1396.50 & 1471.02 \\
\hline & 1482.48 & 1899.99 & 3087.03 & 3118.41 & 3168.63 & 3182.66 & 3799.58 & & & \\
\hline \multirow[t]{3}{*}{$\mathrm{Cj}-\mathrm{CF}-\mathrm{C}(=\mathrm{O}) \mathrm{OH}$} & 64.92 & 179.89 & 263.61 & 322.26 & 403.19 & 501.06 & 545.93 & 579.10 & 623.35 & 747.72 \\
\hline & 853.54 & 942.73 & 1078.24 & 1145.40 & 1216.84 & 1275.89 & 1310.68 & 1391.02 & 1419.54 & 1904.92 \\
\hline & 3026.50 & 3218.34 & 3338.51 & 3794.27 & & & & & & \\
\hline \multirow[t]{3}{*}{$\mathrm{C}-\mathrm{CjF}-\mathrm{C}(=\mathrm{O}) \mathrm{OH}$} & 115.82 & 133.56 & 239.18 & 280.49 & 355.77 & 531.80 & 532.93 & 542.21 & 715.94 & 766.74 \\
\hline & 974.17 & 997.17 & 1144.81 & 1250.04 & 1344.12 & 1415.40 & 1442.40 & 1449.34 & 1530.30 & 1758.81 \\
\hline & 3051.13 & 3101.95 & 3191.60 & 3830.79 & & & & & & \\
\hline
\end{tabular}




\begin{tabular}{|c|c|c|c|c|c|c|c|c|c|c|}
\hline \multirow[t]{2}{*}{$\mathrm{C}-\mathrm{Cj}-\mathrm{C}(=\mathrm{O}) \mathrm{OH}$} & 100.85 & 150.70 & 258.85 & 476.34 & 518.87 & 604.64 & 616.31 & 748.44 & 841.77 & 979.57 \\
\hline & 1049.93 & 1136.25 & 1172.84 & 1290.27 & 1385.32 & 1438.14 & 1456.47 & 1505.54 & 1759.94 & 3039.79 \\
\hline \multirow{4}{*}{$\mathrm{C}-\mathrm{CF}-\mathrm{C}(=\mathrm{O}) \mathrm{Oj}$} & 3082.51 & 3168.94 & 3243.50 & 3827.53 & & & & & & \\
\hline & 54.53 & 172.19 & 219.42 & 243.70 & 332.06 & 393.66 & 511.98 & 659.51 & 782.18 & 918.46 \\
\hline & 1056.20 & 1128.31 & 1175.37 & 1259.82 & 1330.58 & 1345.10 & 1394.24 & 1466.67 & 1480.34 & 1644.16 \\
\hline & 3086.98 & 3093.27 & 3171.39 & 3177.26 & & & & & & \\
\hline \multirow[t]{3}{*}{$\mathrm{CF} 2-\mathrm{C}-\mathrm{C}(=\mathrm{O}) \mathrm{OH}$} & 45.46 & 88.51 & 167.36 & 355.97 & 362.25 & 509.08 & 517.04 & 562.68 & 641.51 & 671.98 \\
\hline & 905.37 & 916.01 & 1073.17 & 1128.97 & 1158.62 & 1190.66 & 1225.60 & 1275.80 & 1377.16 & 1404.42 \\
\hline & 1435.59 & 1452.41 & 1866.25 & 3106.97 & 3150.66 & 3165.46 & 3807.66 & & & \\
\hline \multirow[t]{3}{*}{$\mathrm{CjF} 2-\mathrm{C}-\mathrm{C}(=\mathrm{O}) \mathrm{OH}$} & 47.43 & 81.76 & 155.77 & 338.80 & 363.21 & 458.01 & 520.34 & 559.40 & 647.86 & 657.42 \\
\hline & 900.20 & 919.64 & 991.50 & 1154.56 & 1180.60 & 1233.20 & 1327.53 & 1360.83 & 1423.43 & 1438.76 \\
\hline & 1880.06 & 3049.02 & 3141.90 & 3812.29 & & & & & & \\
\hline \multirow[t]{3}{*}{$\mathrm{CF} 2-\mathrm{Cj}-\mathrm{C}(=\mathrm{O}) \mathrm{OH}$} & 36.73 & 85.35 & 190.94 & 348.43 & 364.79 & 494.37 & 518.01 & 559.49 & 621.15 & 648.29 \\
\hline & 766.79 & 982.35 & 1102.00 & 1115.75 & 1144.52 & 1180.31 & 1255.91 & 1369.85 & 1395.72 & 1491.92 \\
\hline & 1755.23 & 3169.96 & 3267.29 & 3821.59 & & & & & & \\
\hline \multirow[t]{3}{*}{$\mathrm{CF} 2-\mathrm{C}-\mathrm{C}(=\mathrm{O}) \mathrm{Oj}$} & 29.30 & 77.58 & 127.17 & 161.36 & 356.13 & 426.90 & 503.77 & 555.19 & 613.48 & 877.74 \\
\hline & 899.48 & 1042.33 & 1141.91 & 1151.41 & 1207.32 & 1250.49 & 1348.66 & 1394.14 & 1424.37 & 1430.88 \\
\hline & 1689.67 & 3105.65 & 3152.25 & 3165.67 & & & & & & \\
\hline \multirow[t]{3}{*}{$\mathrm{CF}-\mathrm{CF}-\mathrm{C}(=\mathrm{O}) \mathrm{OH}$} & 59.29 & 106.27 & 189.13 & 270.73 & 340.18 & 495.13 & 549.65 & 589.11 & 615.52 & 784.89 \\
\hline & 878.37 & 915.41 & 1031.25 & 1119.85 & 1160.29 & 1216.57 & 1263.83 & 1312.53 & 1343.55 & 1389.98 \\
\hline & 1417.62 & 1475.31 & 1904.58 & 3105.59 & 3132.33 & 3165.52 & 3805.01 & & & \\
\hline \multirow[t]{3}{*}{$\mathrm{CjF}-\mathrm{CF}-\mathrm{C}(=\mathrm{O}) \mathrm{OH}$} & 61.62 & 88.62 & 191.43 & 232.64 & 299.64 & 373.48 & 465.87 & 521.24 & 604.93 & 641.19 \\
\hline & 733.76 & 930.82 & 1004.85 & 1128.45 & 1202.30 & 1229.54 & 1254.09 & 1308.84 & 1395.70 & 1455.40 \\
\hline & 1903.04 & 3080.74 & 3295.46 & 3787.68 & & & & & & \\
\hline \multirow[t]{3}{*}{$\mathrm{CF}-\mathrm{CjF}-\mathrm{C}(=\mathrm{O}) \mathrm{OH}$} & 56.15 & 94.16 & 191.22 & 229.36 & 341.15 & 415.07 & 527.23 & 541.84 & 613.88 & 724.08 \\
\hline & 794.70 & 969.52 & 1073.33 & 1150.27 & 1207.91 & 1278.69 & 1360.15 & 1423.32 & 1471.22 & 1513.70 \\
\hline & 1766.45 & 3084.54 & 3174.22 & 3826.26 & & & & & & \\
\hline \multirow[t]{3}{*}{$\mathrm{CF}-\mathrm{CF}-\mathrm{C}(=\mathrm{O}) \mathrm{Oj}$} & 57.85 & 102.90 & 178.52 & 201.38 & 266.29 & 348.85 & 497.05 & 539.28 & 710.69 & 858.44 \\
\hline & 909.50 & 1029.54 & 1138.60 & 1185.42 & 1265.76 & 1299.56 & 1336.54 & 1350.89 & 1404.93 & 1472.76 \\
\hline & 1677.66 & 3096.00 & 3105.75 & 3157.95 & & & & & & \\
\hline \multirow[t]{3}{*}{$\mathrm{C}-\mathrm{CF} 2-\mathrm{C}(=\mathrm{O}) \mathrm{OH}$} & 36.43 & 219.80 & 229.78 & 245.22 & 354.20 & 375.10 & 418.19 & 570.55 & 584.64 & 607.51 \\
\hline & 762.88 & 796.50 & 943.74 & 967.44 & 1165.68 & 1210.81 & 1220.98 & 1271.61 & 1411.53 & 1431.77 \\
\hline & 1458.07 & 1471.65 & 1882.13 & 3102.44 & 3193.35 & 3195.74 & 3812.60 & & & \\
\hline \multirow[t]{3}{*}{$\mathrm{Cj}-\mathrm{CF} 2-\mathrm{C}(=\mathrm{O}) \mathrm{OH}$} & 24.68 & 135.14 & 238.61 & 245.87 & 363.66 & 366.15 & 419.75 & 572.19 & 579.83 & 600.35 \\
\hline & 603.38 & 770.16 & 794.65 & 920.70 & 1105.10 & 1168.90 & 1218.64 & 1273.53 & 1422.50 & 1448.94 \\
\hline & 1880.81 & 3227.65 & 3354.80 & 3813.55 & & & & & & \\
\hline \multirow[t]{3}{*}{$\mathrm{C}-\mathrm{CF} 2-\mathrm{C}(=\mathrm{O}) \mathrm{Oj}$} & 19.94 & 198.47 & 204.32 & 218.00 & 246.22 & 364.70 & 424.65 & 433.40 & 563.74 & 718.54 \\
\hline & 744.46 & 932.78 & 970.77 & 1184.53 & 1218.49 & 1234.84 & 1294.67 & 1404.17 & 1455.01 & 1463.18 \\
\hline & 1736.35 & 3097.86 & 3187.02 & 3197.13 & & & & & & \\
\hline \multirow[t]{3}{*}{ CF3-C-C(=O)OH } & 44.26 & 105.11 & 155.95 & 321.10 & 338.01 & 358.17 & 499.45 & 530.34 & 549.79 & 611.36 \\
\hline & 657.80 & 708.24 & 878.49 & 921.59 & 935.43 & 1139.44 & 1147.56 & 1210.59 & 1285.80 & 1321.28 \\
\hline & 1351.65 & 1429.83 & 1445.89 & 1886.36 & 3121.33 & 3168.22 & 3810.79 & & & \\
\hline \multirow[t]{3}{*}{$\mathrm{CF} 3-\mathrm{Cj}-\mathrm{C}(=\mathrm{O}) \mathrm{OH}$} & 65.98 & 82.96 & 153.63 & 316.76 & 346.77 & 376.66 & 490.75 & 522.99 & 537.34 & 612.75 \\
\hline & 615.92 & 705.10 & 760.62 & 890.06 & 970.82 & 1169.50 & 1189.71 & 1210.39 & 1274.37 & 1389.60 \\
\hline & 1441.09 & 1768.62 & 3294.35 & 3824.35 & & & & & & \\
\hline \multirow[t]{3}{*}{$\mathrm{CF} 3-\mathrm{C}-\mathrm{C}(=\mathrm{O}) \mathrm{Oj}$} & 17.25 & 92.93 & 139.58 & 308.58 & 334.11 & 364.24 & 499.78 & 528.21 & 551.56 & 613.47 \\
\hline & 657.47 & 714.26 & 878.71 & 918.24 & 933.15 & 1143.56 & 1192.90 & 1291.50 & 1316.13 & 1383.04 \\
\hline & 1424.81 & 1639.37 & 3119.85 & 3169.69 & & & & & & \\
\hline \multirow[t]{3}{*}{$\mathrm{CF} 2-\mathrm{CF}-\mathrm{C}(=\mathrm{O}) \mathrm{OH}$} & 43.86 & 70.09 & 144.48 & 248.88 & 269.03 & 376.02 & 403.34 & 497.19 & 582.21 & 597.23 \\
\hline & 673.39 & 763.03 & 939.95 & 1109.30 & 1149.58 & 1171.52 & 1178.32 & 1192.24 & 1260.81 & 1310.40 \\
\hline & 1372.29 & 1417.91 & 1442.71 & 1890.39 & 3130.67 & 3144.44 & 3805.90 & & & \\
\hline
\end{tabular}




\begin{tabular}{|c|c|c|c|c|c|c|c|c|c|c|}
\hline \multirow[t]{3}{*}{$\mathrm{CjF} 2-\mathrm{CF}-\mathrm{C}(=\mathrm{O}) \mathrm{OH}$} & 49.28 & 65.45 & 138.18 & 216.93 & 277.20 & 381.05 & 469.69 & 484.88 & 588.62 & 602.13 \\
\hline & 640.76 & 751.71 & 888.97 & 945.95 & 1152.02 & 1187.00 & 1225.50 & 1300.89 & 1342.03 & 1377.99 \\
\hline & 1420.01 & 1907.51 & 3119.68 & 3815.02 & & & & & & \\
\hline \multirow[t]{3}{*}{$\mathrm{CF} 2-\mathrm{CjF}-\mathrm{C}(=\mathrm{O}) \mathrm{OH}$} & 42.58 & 85.38 & 174.55 & 179.23 & 259.30 & 351.99 & 468.52 & 516.73 & 563.11 & 564.98 \\
\hline & 676.54 & 727.25 & 867.65 & 1096.25 & 1128.78 & 1167.71 & 1197.32 & 1362.59 & 1372.85 & 1439.62 \\
\hline & 1526.80 & 1766.39 & 3176.87 & 3824.46 & & & & & & \\
\hline \multirow[t]{3}{*}{$\mathrm{CF} 2-\mathrm{CF}-\mathrm{C}(=\mathrm{O}) \mathrm{Oj}$} & 55.91 & 71.49 & 148.88 & 210.73 & 237.68 & 259.47 & 416.85 & 492.44 & 513.66 & 626.99 \\
\hline & 794.05 & 898.47 & 934.39 & 1154.96 & 1171.45 & 1224.61 & 1268.89 & 1325.57 & 1355.48 & 1395.55 \\
\hline & 1405.98 & 1699.68 & 3115.30 & 3142.82 & & & & & & \\
\hline \multirow[t]{3}{*}{$\mathrm{CF}-\mathrm{CF} 2-\mathrm{C}(=\mathrm{O}) \mathrm{OH}$} & 27.47 & 98.33 & 176.38 & 242.25 & 266.97 & 361.88 & 411.60 & 483.09 & 548.28 & 603.21 \\
\hline & 630.41 & 761.14 & 798.05 & 955.47 & 1141.36 & 1168.11 & 1188.54 & 1202.54 & 1256.44 & 1308.24 \\
\hline & 1414.53 & 1452.65 & 1475.70 & 1884.99 & 3114.76 & 3182.19 & 3805.71 & & & \\
\hline \multirow[t]{3}{*}{$\mathrm{CjF}-\mathrm{CF} 2-\mathrm{C}(=\mathrm{O}) \mathrm{OH}$} & 42.45 & 81.86 & 161.11 & 242.23 & 265.23 & 337.86 & 389.00 & 419.78 & 464.42 & 569.82 \\
\hline & 611.57 & 655.91 & 775.36 & 797.67 & 1095.28 & 1165.79 & 1203.33 & 1227.23 & 1269.38 & 1389.89 \\
\hline & 1454.10 & 1893.13 & 3280.60 & 3808.85 & & & & & & \\
\hline \multirow[t]{3}{*}{$\mathrm{CF}-\mathrm{CF} 2-\mathrm{C}(=\mathrm{O}) \mathrm{Oj}$} & 34.41 & 94.91 & 165.53 & 211.22 & 230.12 & 275.47 & 405.04 & 418.85 & 496.07 & 565.36 \\
\hline & 710.81 & 760.62 & 944.46 & 1146.74 & 1174.70 & 1206.75 & 1219.78 & 1285.21 & 1303.37 & 1410.06 \\
\hline & 1473.13 & 1753.60 & 3109.74 & 3176.34 & & & & & & \\
\hline \multirow[t]{3}{*}{$\mathrm{CF} 3-\mathrm{CF}-\mathrm{C}(=\mathrm{O}) \mathrm{OH}$} & 35.88 & 67.78 & 149.16 & 217.20 & 261.61 & 323.05 & 367.98 & 485.42 & 524.17 & 536.05 \\
\hline & 599.07 & 632.62 & 684.08 & 769.88 & 894.75 & 939.81 & 1182.37 & 1186.99 & 1199.12 & 1274.32 \\
\hline & 1307.57 & 1327.86 & 1380.24 & 1429.19 & 1882.31 & 3142.66 & 3807.18 & & & \\
\hline \multirow[t]{3}{*}{$\mathrm{CF} 3-\mathrm{CjF}-\mathrm{C}(=\mathrm{O}) \mathrm{OH}$} & 40.31 & 83.14 & 149.92 & 175.30 & 283.09 & 354.50 & 371.08 & 392.97 & 511.48 & 533.62 \\
\hline & 579.16 & 612.70 & 652.18 & 726.03 & 783.73 & 1064.07 & 1174.44 & 1229.38 & 1251.63 & 1299.39 \\
\hline & 1425.30 & 1518.71 & 1787.18 & 3821.63 & & & & & & \\
\hline \multirow[t]{3}{*}{$\mathrm{CF} 3-\mathrm{CF}-\mathrm{C}(=\mathrm{O}) \mathrm{Oj}$} & 38.50 & 61.49 & 133.31 & 172.97 & 223.91 & 262.64 & 363.17 & 381.27 & 512.57 & 527.04 \\
\hline & 542.38 & 613.92 & 731.25 & 880.42 & 914.57 & 1178.93 & 1190.50 & 1224.48 & 1275.49 & 1313.03 \\
\hline & 1325.10 & 1382.20 & 1648.87 & 3131.43 & & & & & & \\
\hline \multirow[t]{3}{*}{$\mathrm{CF} 2-\mathrm{CF} 2-\mathrm{C}(=\mathrm{O}) \mathrm{OH}$} & 30.09 & 82.17 & 148.04 & 223.39 & 239.32 & 278.26 & 361.68 & 413.48 & 449.47 & 565.32 \\
\hline & 589.05 & 626.30 & 685.53 & 791.75 & 851.93 & 1088.84 & 1175.80 & 1185.94 & 1198.88 & 1214.99 \\
\hline & 1316.18 & 1374.35 & 1420.62 & 1453.74 & 1882.95 & 3167.53 & 3802.77 & & & \\
\hline \multirow[t]{3}{*}{$\mathrm{CjF} 2-\mathrm{CF} 2-\mathrm{C}(=\mathrm{O}) \mathrm{OH}$} & 23.47 & 65.22 & 132.46 & 200.28 & 242.25 & 346.81 & 364.90 & 404.48 & 467.49 & 549.14 \\
\hline & 576.82 & 613.65 & 641.17 & 751.42 & 801.41 & 1055.12 & 1164.32 & 1212.51 & 1269.22 & 1348.13 \\
\hline & 1373.61 & 1420.39 & 1904.89 & 3809.03 & & & & & & \\
\hline \multirow[t]{3}{*}{$\mathrm{CF} 2-\mathrm{CF} 2-\mathrm{C}(=\mathrm{O}) \mathrm{Oj}$} & 32.18 & 74.25 & 126.52 & 188.16 & 215.59 & 243.68 & 273.19 & 393.93 & 449.81 & 500.12 \\
\hline & 560.45 & 625.39 & 745.69 & 840.34 & 1070.67 & 1174.57 & 1186.73 & 1214.80 & 1238.80 & 1309.19 \\
\hline & 1376.94 & 1408.87 & 1776.46 & 3162.60 & & & & & & \\
\hline \multirow[t]{3}{*}{$\mathrm{CF} 3-\mathrm{CF} 2-\mathrm{C}(=\mathrm{O}) \mathrm{OH}$} & 21.76 & 67.06 & 141.05 & 206.79 & 245.39 & 278.52 & 352.90 & 372.79 & 377.07 & 501.93 \\
\hline & 534.02 & 545.05 & 608.54 & 628.83 & 675.51 & 780.45 & 789.21 & 1051.98 & 1169.84 & 1221.17 \\
\hline & 1271.67 & 1288.41 & 1293.60 & 1367.01 & 1431.02 & 1908.37 & 3807.29 & & & \\
\hline \multirow[t]{3}{*}{$\mathrm{CF} 3-\mathrm{CF} 2-\mathrm{C}(=\mathrm{O}) \mathrm{Oj}$} & 28.08 & 63.85 & 132.85 & 194.33 & 216.54 & 242.03 & 277.11 & 357.73 & 373.56 & 441.45 \\
\hline & 519.72 & 551.18 & 577.44 & 612.43 & 741.30 & 780.78 & 1045.32 & 1210.48 & 1253.94 & 1289.12 \\
\hline & 1297.96 & 1310.20 & 1375.68 & 1736.51 & & & & & & \\
\hline
\end{tabular}




\section{Moments of Inertia}

In this article, explicit hydrogens are not included in species but are implied unless otherwise stated. And $\mathrm{j}$ represents a radical site on the preceding carbon or oxygen atom.

From MN15/cc-pVTZ level of theory

Table S2 Moments of Inertia (AMU Bohr²)

\begin{tabular}{|c|c|c|c|}
\hline Species & $I_{a}$ & $I_{b}$ & $I_{c}$ \\
\hline $\mathrm{CF}-\mathrm{C}(=\mathrm{O}) \mathrm{OH}$ & 167.21239 & 458.46387 & 614.39358 \\
\hline $\mathrm{CjF}-\mathrm{C}(=\mathrm{O}) \mathrm{OH}$ & 163.07771 & 432.01827 & 595.09597 \\
\hline $\mathrm{Cj}-\mathrm{C}(=\mathrm{O}) \mathrm{OH}$ & 153.19793 & 169.77103 & 322.96896 \\
\hline $\mathrm{CF}-\mathrm{C}(=\mathrm{O}) \mathrm{Oj}$ & 152.56567 & 443.38011 & 584.59041 \\
\hline $\mathrm{CF} 2-\mathrm{C}(=\mathrm{O}) \mathrm{OH}$ & 320.60320 & 657.28497 & 659.84916 \\
\hline $\mathrm{CjF} 2-\mathrm{C}(=\mathrm{O}) \mathrm{OH}$ & 311.87728 & 527.00517 & 838.88245 \\
\hline $\mathrm{CF} 2-\mathrm{C}(=\mathrm{O}) \mathrm{Oj}$ & 313.53771 & 516.07909 & 791.67755 \\
\hline $\mathrm{CF} 3-\mathrm{C}(=\mathrm{O}) \mathrm{OH}$ & 464.44561 & 717.75739 & 866.24536 \\
\hline $\mathrm{CF} 3-\mathrm{C}(=\mathrm{O}) \mathrm{Oj}$ & 453.01586 & 710.93638 & 848.48525 \\
\hline $\mathrm{CF}-\mathrm{C}-\mathrm{C}(=\mathrm{O}) \mathrm{OH}$ & 186.23650 & 981.74588 & 1145.72972 \\
\hline $\mathrm{CjF}-\mathrm{C}-\mathrm{C}(=\mathrm{O}) \mathrm{OH}$ & 177.46369 & 970.23658 & 1135.82711 \\
\hline $\mathrm{Cj}-\mathrm{C}-\mathrm{C}(=\mathrm{O}) \mathrm{OH}$ & 171.09689 & 441.89515 & 602.24539 \\
\hline $\mathrm{CF}-\mathrm{Cj}-\mathrm{C}(=\mathrm{O}) \mathrm{OH}$ & 179.00931 & 975.76978 & 1143.67207 \\
\hline $\mathrm{CF}-\mathrm{C}-\mathrm{C}(=\mathrm{O}) \mathrm{Oj}$ & 250.70101 & 773.90049 & 829.08471 \\
\hline $\mathrm{C}-\mathrm{CF}-\mathrm{C}(=\mathrm{O}) \mathrm{OH}$ & 355.53687 & 530.48540 & 803.52970 \\
\hline $\mathrm{Cj}-\mathrm{CF}-\mathrm{C}(=\mathrm{O}) \mathrm{OH}$ & 341.03796 & 514.13060 & 791.55458 \\
\hline $\mathrm{C}-\mathrm{CjF}-\mathrm{C}(=\mathrm{O}) \mathrm{OH}$ & 333.70997 & 532.42342 & 854.97742 \\
\hline $\mathrm{C}-\mathrm{Cj}-\mathrm{C}(=\mathrm{O}) \mathrm{OH}$ & 165.04013 & 466.20080 & 620.24178 \\
\hline $\mathrm{C}-\mathrm{CF}-\mathrm{C}(=\mathrm{O}) \mathrm{Oj}$ & 331.32656 & 534.28111 & 797.47805 \\
\hline $\mathrm{CF} 2-\mathrm{C}-\mathrm{C}(=\mathrm{O}) \mathrm{OH}$ & 323.04033 & 1171.81552 & 1290.14236 \\
\hline $\mathrm{CjF} 2-\mathrm{C}-\mathrm{C}(=\mathrm{O}) \mathrm{OH}$ & 310.82566 & 1149.32652 & 1327.46676 \\
\hline $\mathrm{CF} 2-\mathrm{Cj}-\mathrm{C}(=\mathrm{O}) \mathrm{OH}$ & 324.59525 & 1292.39784 & 1294.16376 \\
\hline $\mathrm{CF} 2-\mathrm{C}-\mathrm{C}(=\mathrm{O}) \mathrm{Oj}$ & 322.29463 & 1156.28852 & 1212.47842 \\
\hline $\mathrm{CF}-\mathrm{CF}-\mathrm{C}(=\mathrm{O}) \mathrm{OH}$ & 521.93447 & 765.24764 & 953.44111 \\
\hline $\mathrm{CjF}-\mathrm{CF}-\mathrm{C}(=\mathrm{O}) \mathrm{OH}$ & 391.79681 & 952.06945 & 1288.48917 \\
\hline $\mathrm{CF}-\mathrm{CjF}-\mathrm{C}(=\mathrm{O}) \mathrm{OH}$ & 401.45065 & 949.24432 & 1260.04479 \\
\hline $\mathrm{CF}-\mathrm{CF}-\mathrm{C}(=\mathrm{O}) \mathrm{Oj}$ & 502.54359 & 752.65780 & 968.61260 \\
\hline $\mathrm{C}-\mathrm{CF} 2-\mathrm{C}(=\mathrm{O}) \mathrm{OH}$ & 491.36266 & 726.72413 & 884.78977 \\
\hline $\mathrm{Cj}-\mathrm{CF} 2-\mathrm{C}(=\mathrm{O}) \mathrm{OH}$ & 476.37138 & 714.26211 & 870.60541 \\
\hline $\mathrm{C}-\mathrm{CF} 2-\mathrm{C}(=\mathrm{O}) \mathrm{Oj}$ & 482.99850 & 717.45190 & 862.51421 \\
\hline $\mathrm{CF} 3-\mathrm{C}-\mathrm{C}(=\mathrm{O}) \mathrm{OH}$ & 450.95307 & 1329.62411 & 1453.38562 \\
\hline $\mathrm{CF} 3-\mathrm{Cj}-\mathrm{C}(=\mathrm{O}) \mathrm{OH}$ & 448.36836 & 1310.03902 & 1443.32072 \\
\hline $\mathrm{CF} 3-\mathrm{C}-\mathrm{C}(=\mathrm{O}) \mathrm{Oj}$ & 432.17229 & 1301.15113 & 1406.31577 \\
\hline $\mathrm{CF} 2-\mathrm{CF}-\mathrm{C}(=\mathrm{O}) \mathrm{OH}$ & 599.32299 & 1103.90774 & 1446.96663 \\
\hline $\mathrm{CjF} 2-\mathrm{CF}-\mathrm{C}(=\mathrm{O}) \mathrm{OH}$ & 650.34869 & 1018.39699 & 1272.88909 \\
\hline $\mathrm{CF} 2-\mathrm{CjF}-\mathrm{C}(=\mathrm{O}) \mathrm{OH}$ & 515.75354 & 1305.04413 & 1499.33221 \\
\hline
\end{tabular}




\begin{tabular}{cccc} 
CF2-CF-C $(=\mathrm{O}) \mathrm{Oj}$ & 527.61185 & 1176.94979 & 1346.00289 \\
CF-CF2-C(=O)OH & 740.14884 & 946.33811 & 1100.85554 \\
CjF-CF2-C $(=\mathrm{O}) \mathrm{OH}$ & 715.48346 & 913.41183 & 1156.23948 \\
\hline CF-CF2-C $(=\mathrm{O}) \mathrm{Oj}$ & 743.25808 & 921.12538 & 1059.02983 \\
CF3-CF-C $(=\mathbf{O}) \mathbf{O H}$ & 729.65801 & 1295.73124 & 1563.82764 \\
CF3-CjF-C $=\mathrm{O}) \mathrm{OH}$ & 687.41721 & 1358.48033 & 1731.81505 \\
CF3-CF-C $=\mathrm{O}) \mathrm{Oj}$ & 702.57868 & 1279.11300 & 1588.69204 \\
CF2-CF2-C(=O)OH & 742.05896 & 1322.33692 & 1493.97523 \\
CjF2-CF2-C $(=\mathrm{O}) \mathrm{OH}$ & 899.91042 & 1218.94685 & 1275.17134 \\
CF2-CF2-C $(=\mathrm{O}) \mathrm{Oj}$ & 753.48746 & 1292.48990 & 1425.33122 \\
CF3-CF2-C(=O)OH & 952.17724 & 1529.02918 & 1581.74326 \\
CF3-CF2-C $(=\mathrm{O}) \mathrm{Oj}$ & 938.95327 & 1522.94048 & 1546.96767 \\
\hline
\end{tabular}

${ }^{a}$ Units in AMU Bohr ${ }^{2}$ 


\section{Hindered Internal Rotor Potential Energy Diagrams}

In this article, explicit hydrogens are not included in species but are implied unless otherwise stated. And $\mathrm{j}$ represents a radical site on the preceding carbon or oxygen atom.

Calculated from the MN15/cc-pVTZ level of theory. Dihedral angle of $0^{\circ}$ and Internal Rotor (IR) notation corresponds to the figures from the Optimized Structures section. For the ethanoic acid fluorinated species $\left(\mathrm{C}_{2} \mathrm{C}_{1}(=\mathrm{O}) \mathrm{OH}\right)$ IR 1 corresponds to rotation around the $\mathrm{C}_{2}--\mathrm{C}_{1}(=\mathrm{O}) \mathrm{OH}$ bond and IR 2 for the $\mathrm{C}_{2} \mathrm{C}_{1}(=\mathrm{O})--\mathrm{OH}$ bond. For the propanoic acid fluorinated species $\left(\mathrm{C}_{3} \mathrm{C}_{2} \mathrm{C}_{1}(=\mathrm{O}) \mathrm{OH}\right)$ IR 1 corresponds to rotation around the $\mathrm{C}_{3}--\mathrm{C}_{2} \mathrm{C}_{1}(=\mathrm{O}) \mathrm{OH}$ bond, IR 2 for the $\mathrm{C}_{3} \mathrm{C}_{2}--\mathrm{C}_{1}(=\mathrm{O}) \mathrm{OH}$ bond, and IR 3 for the $\mathrm{C}_{3} \mathrm{C}_{2} \mathrm{C}_{1}(=\mathrm{O})--\mathrm{OH}$ bond.
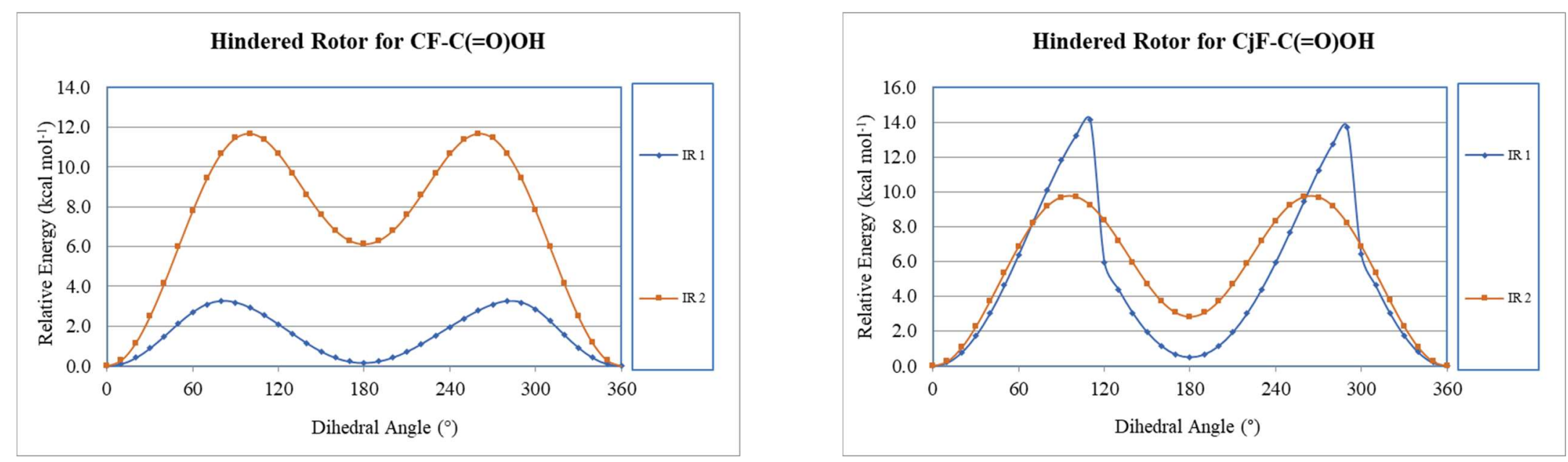

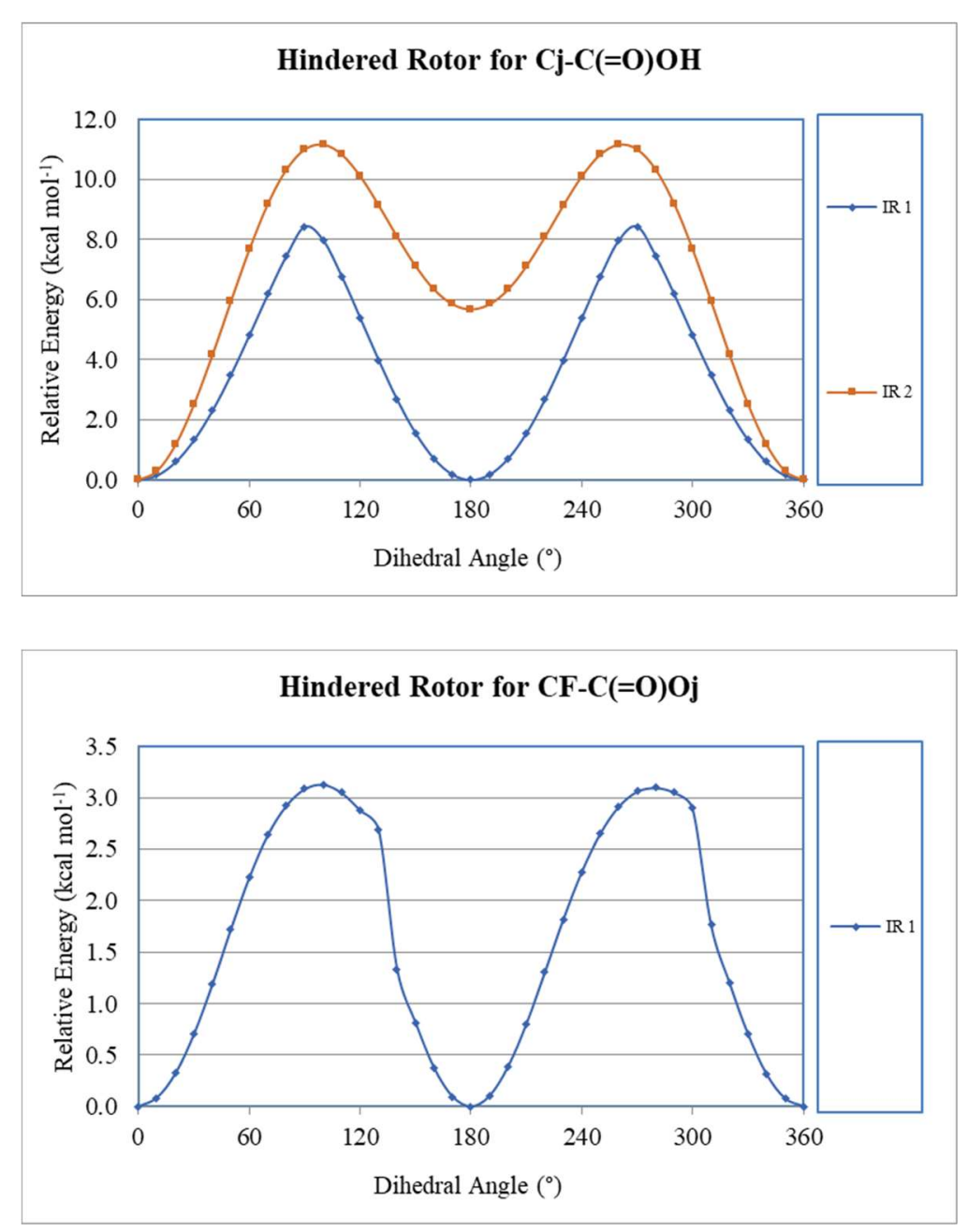
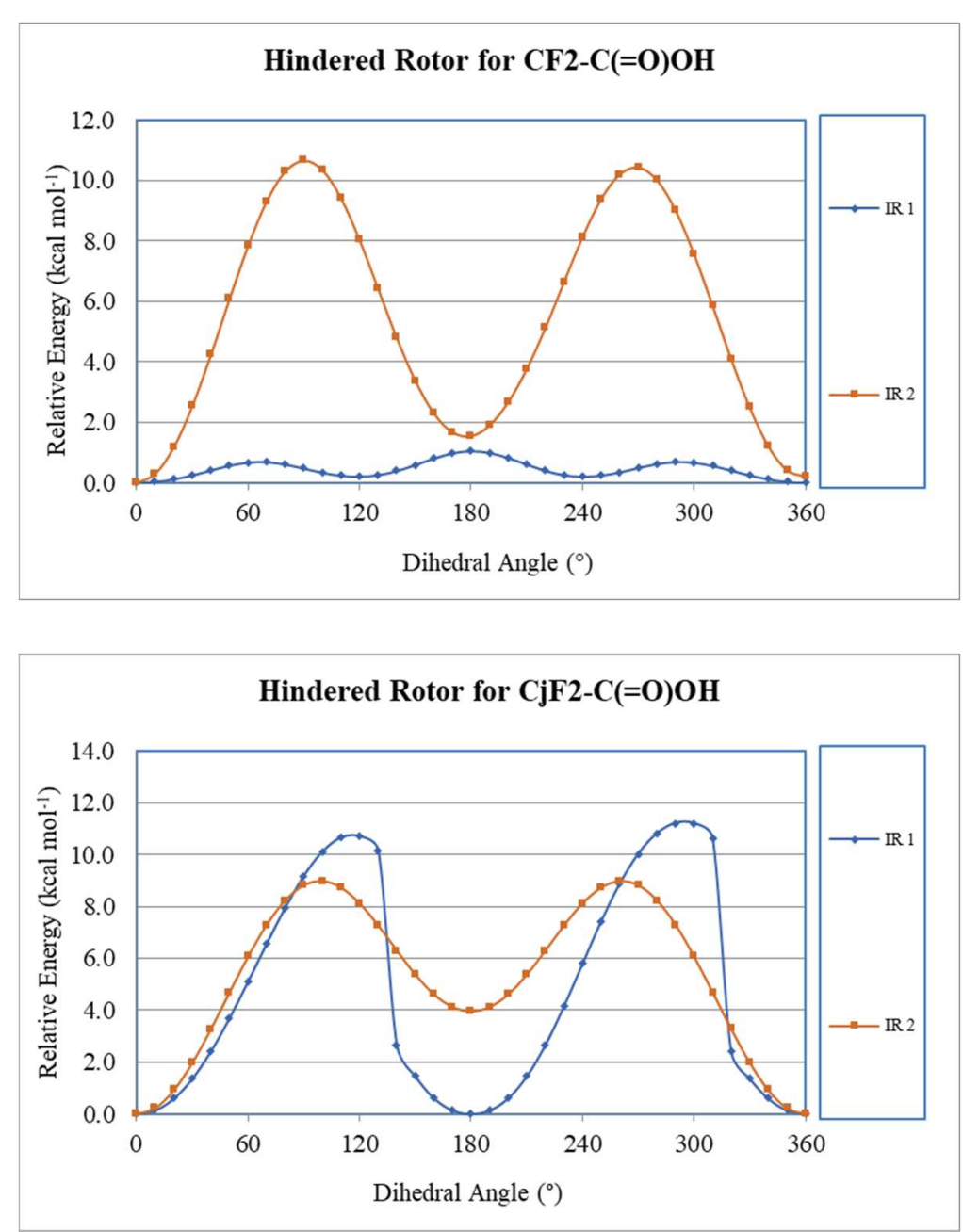

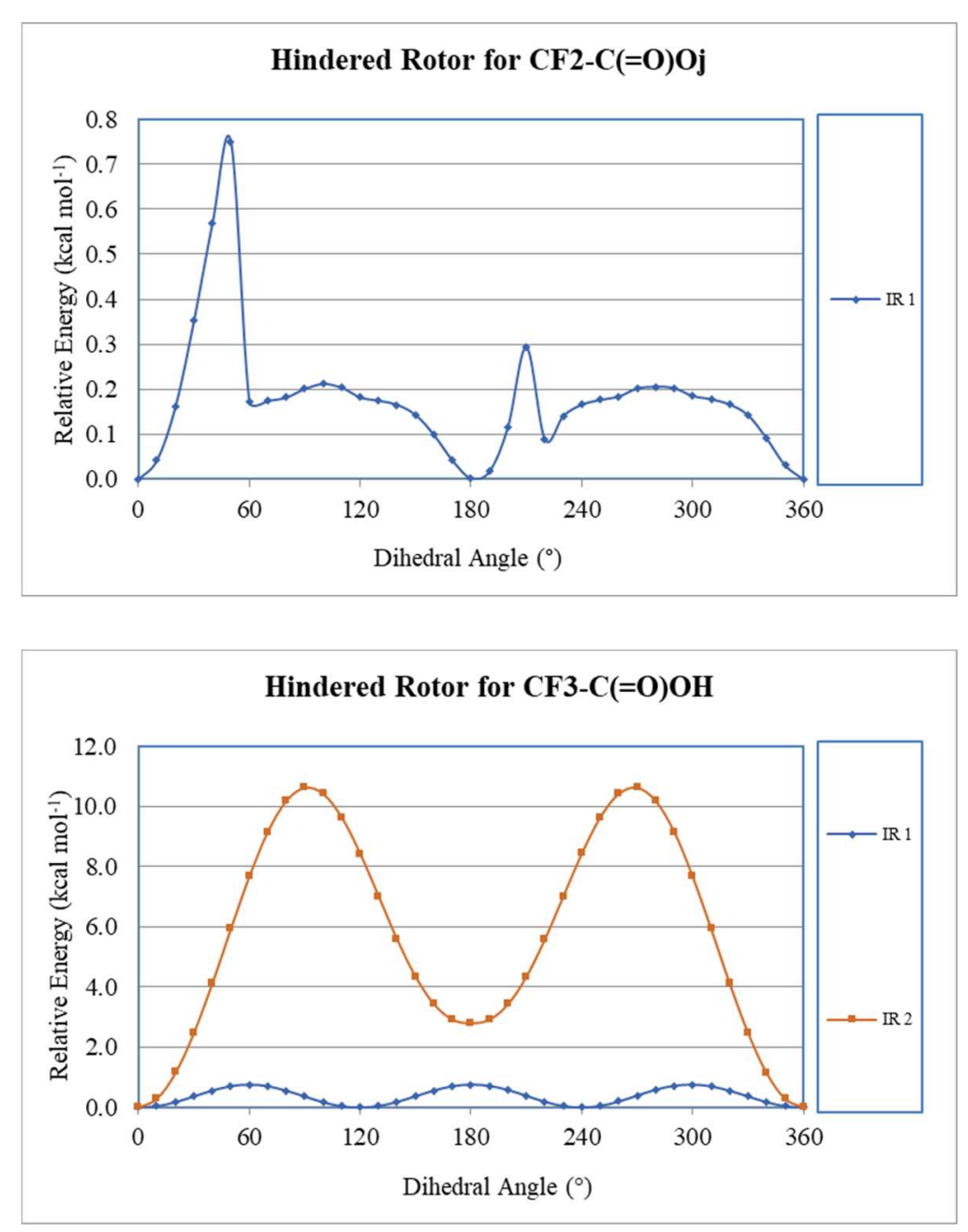
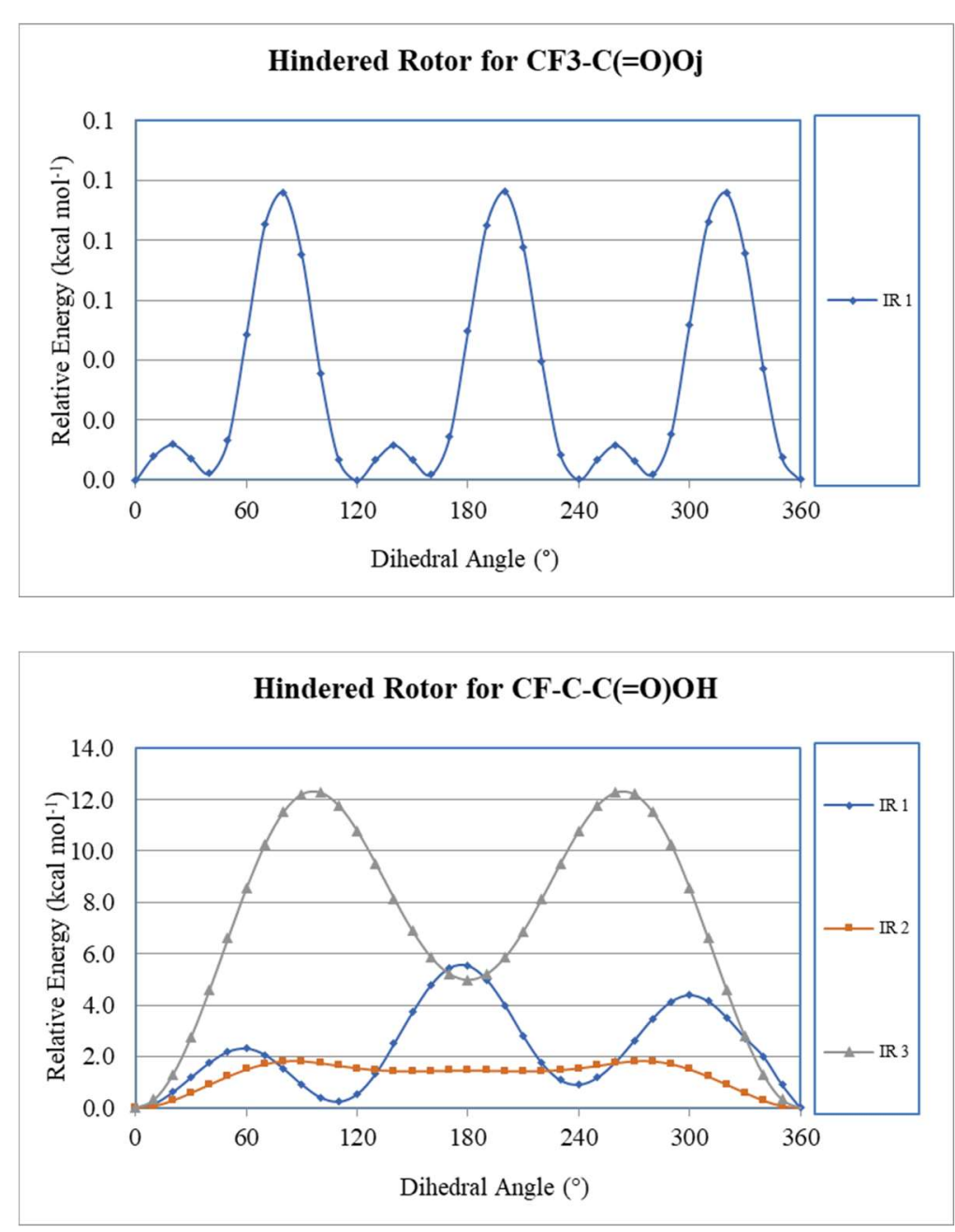

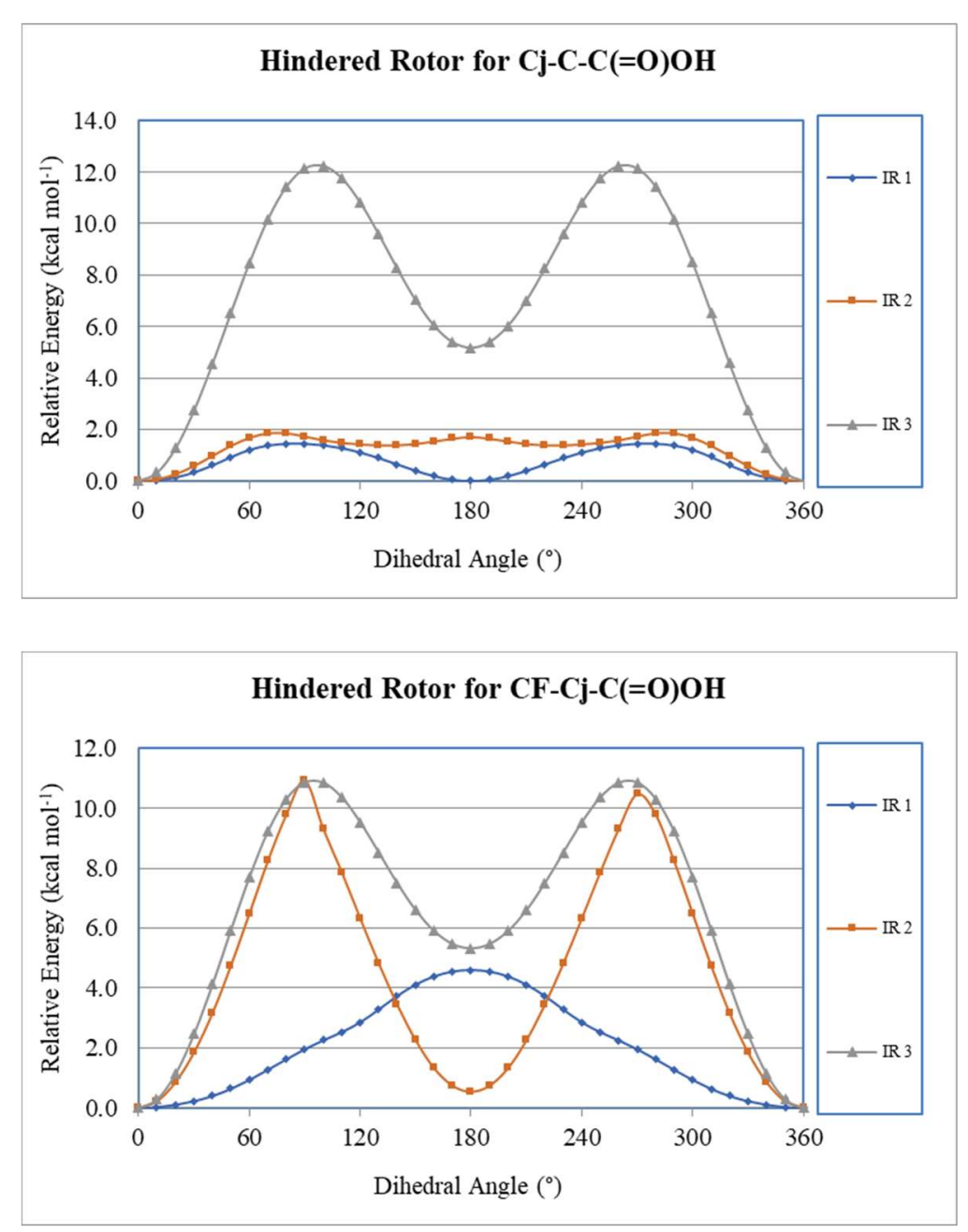
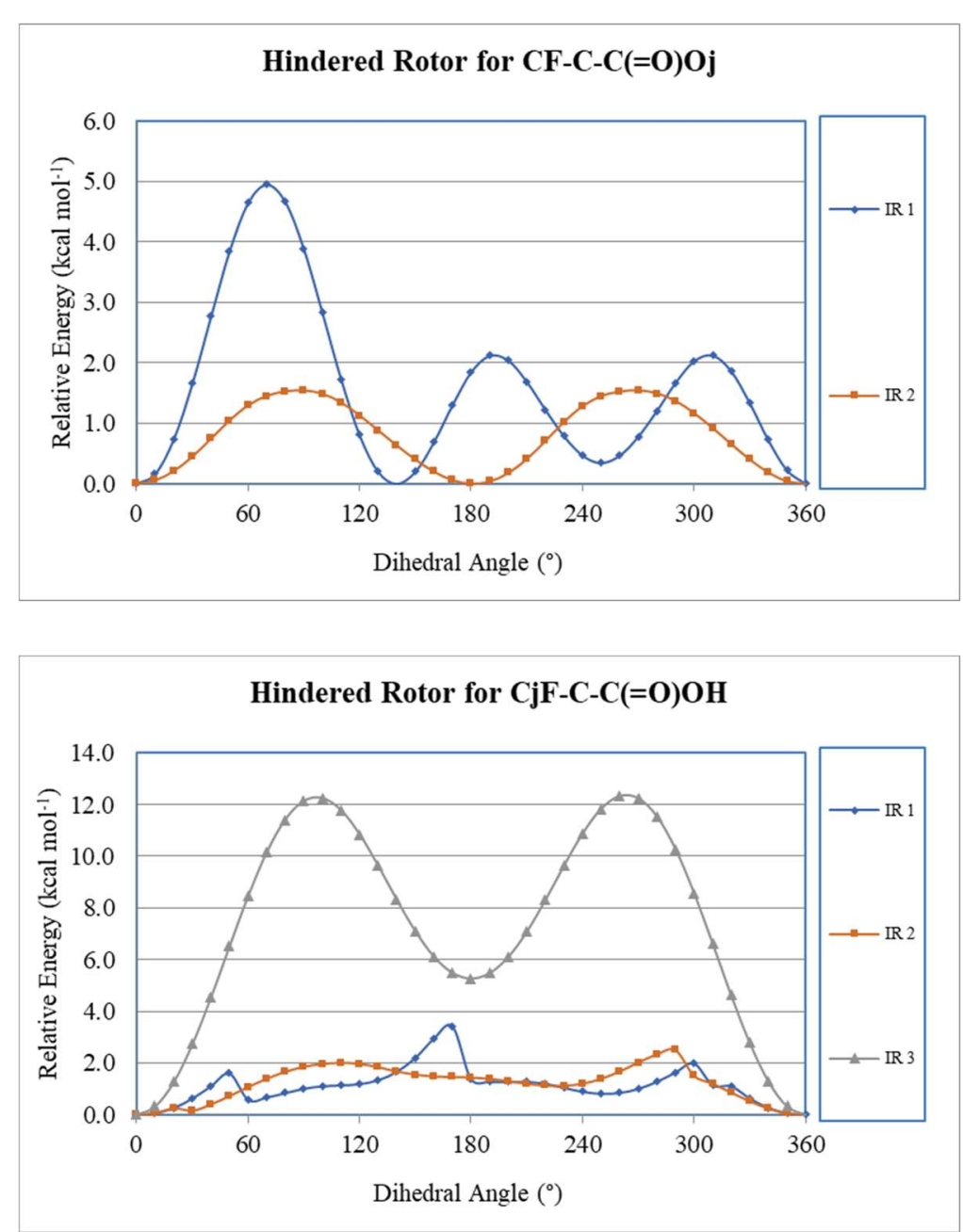

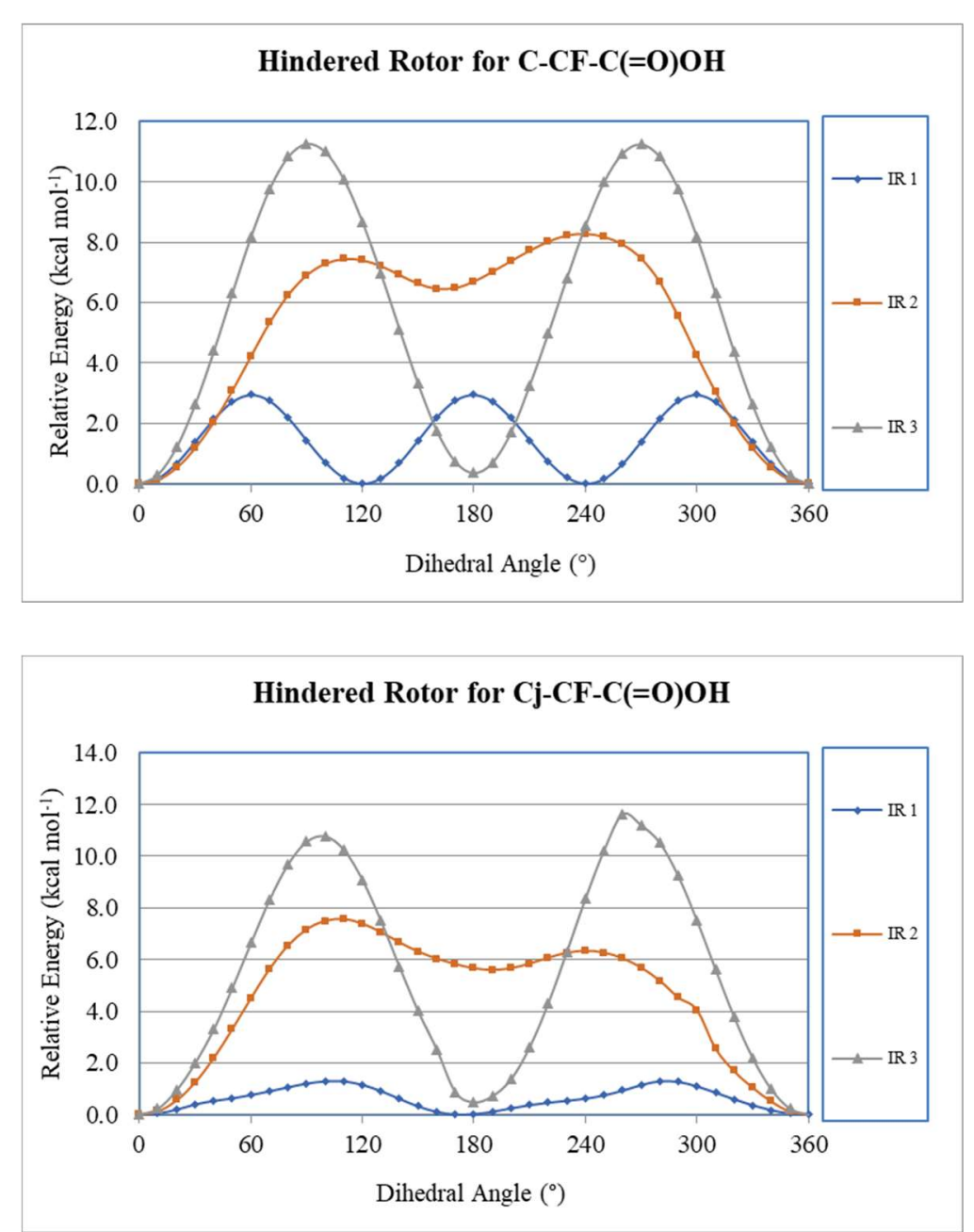
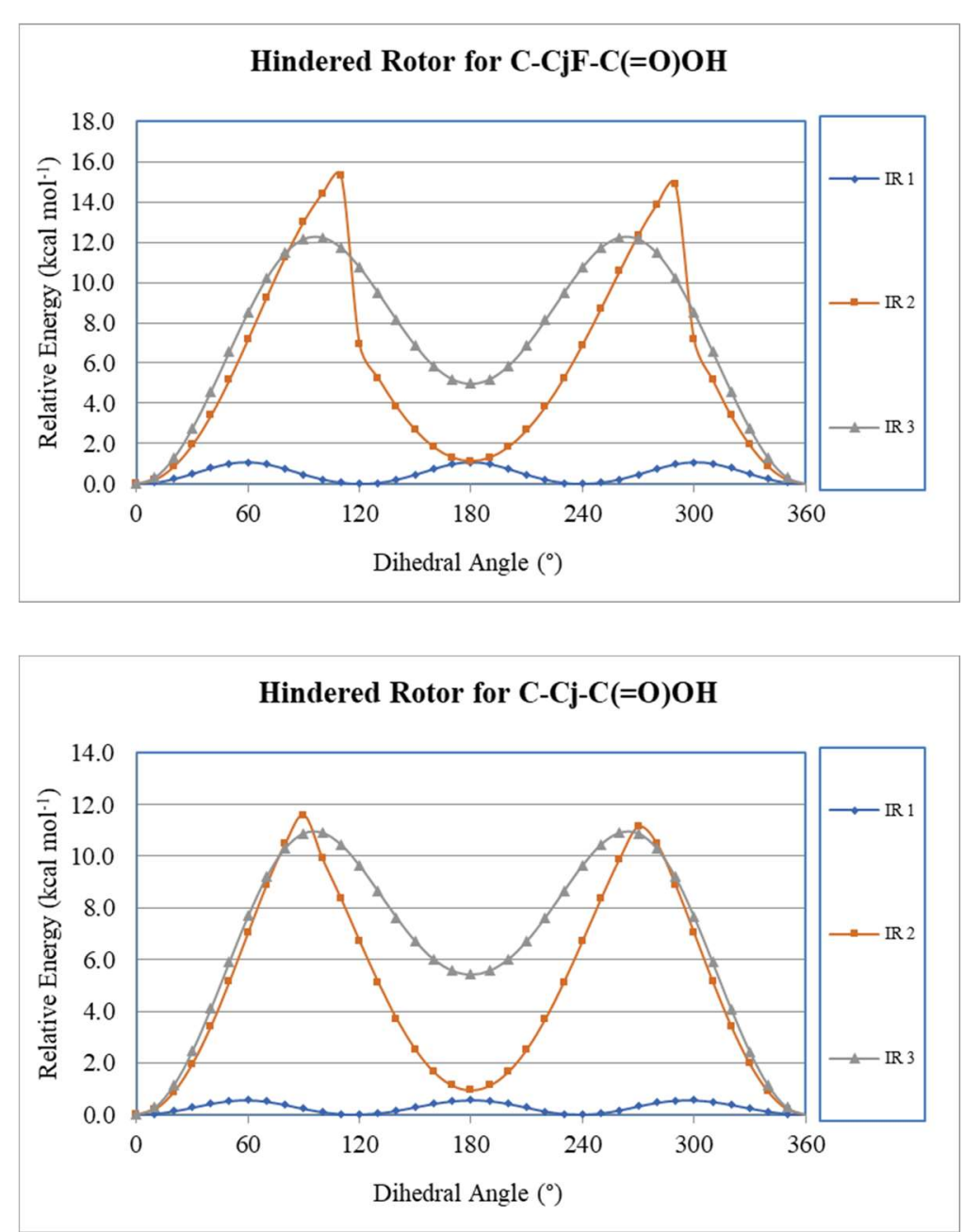

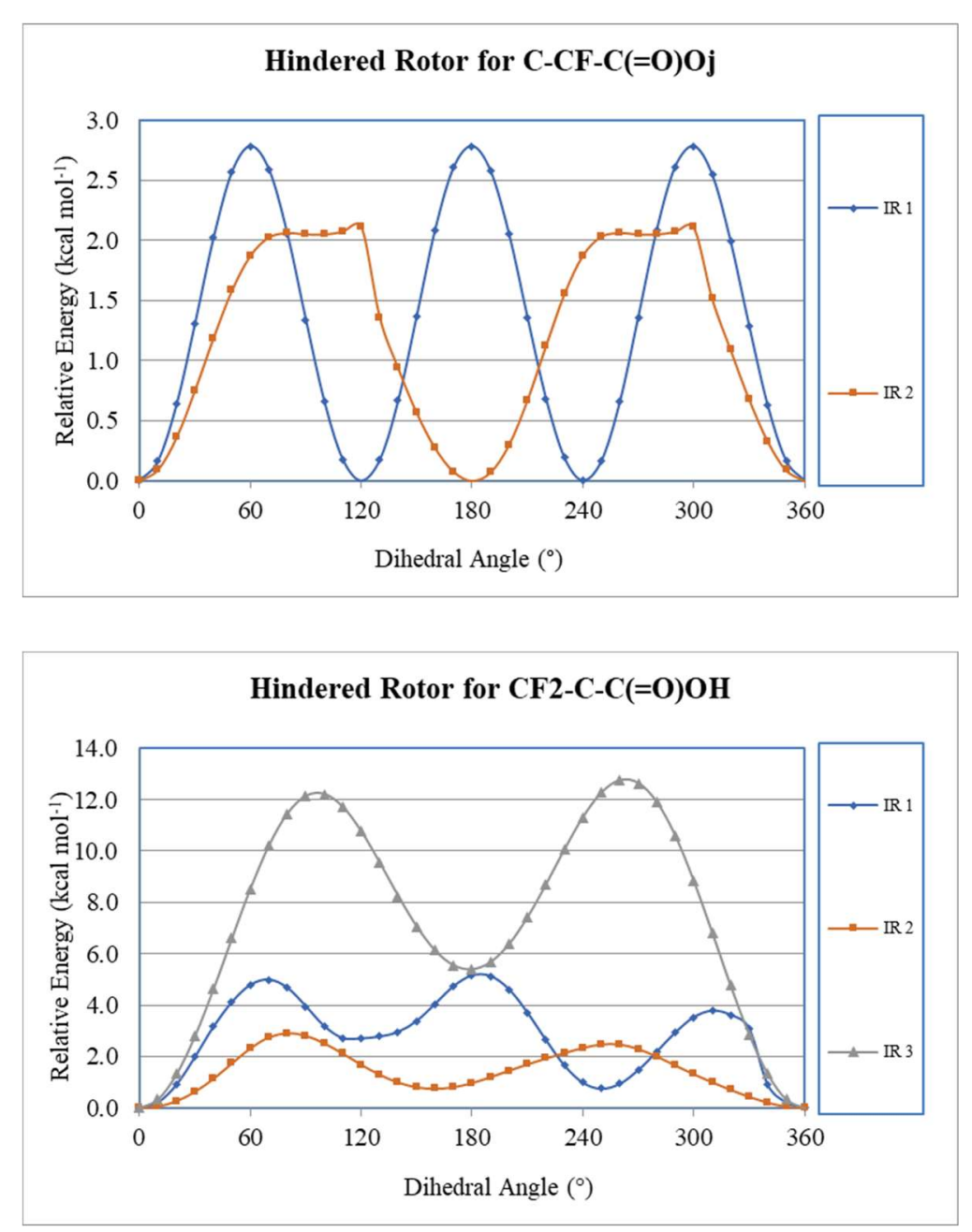
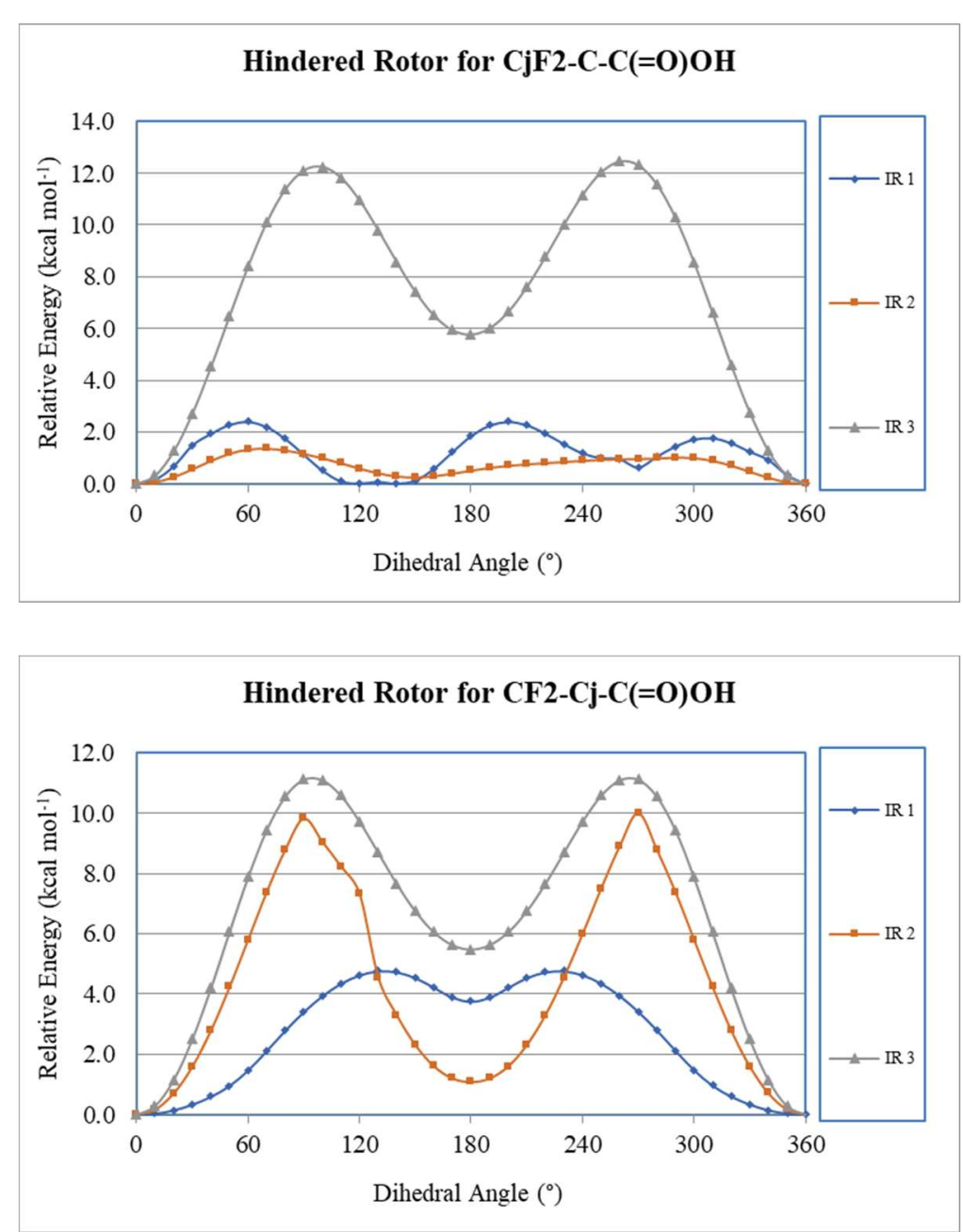

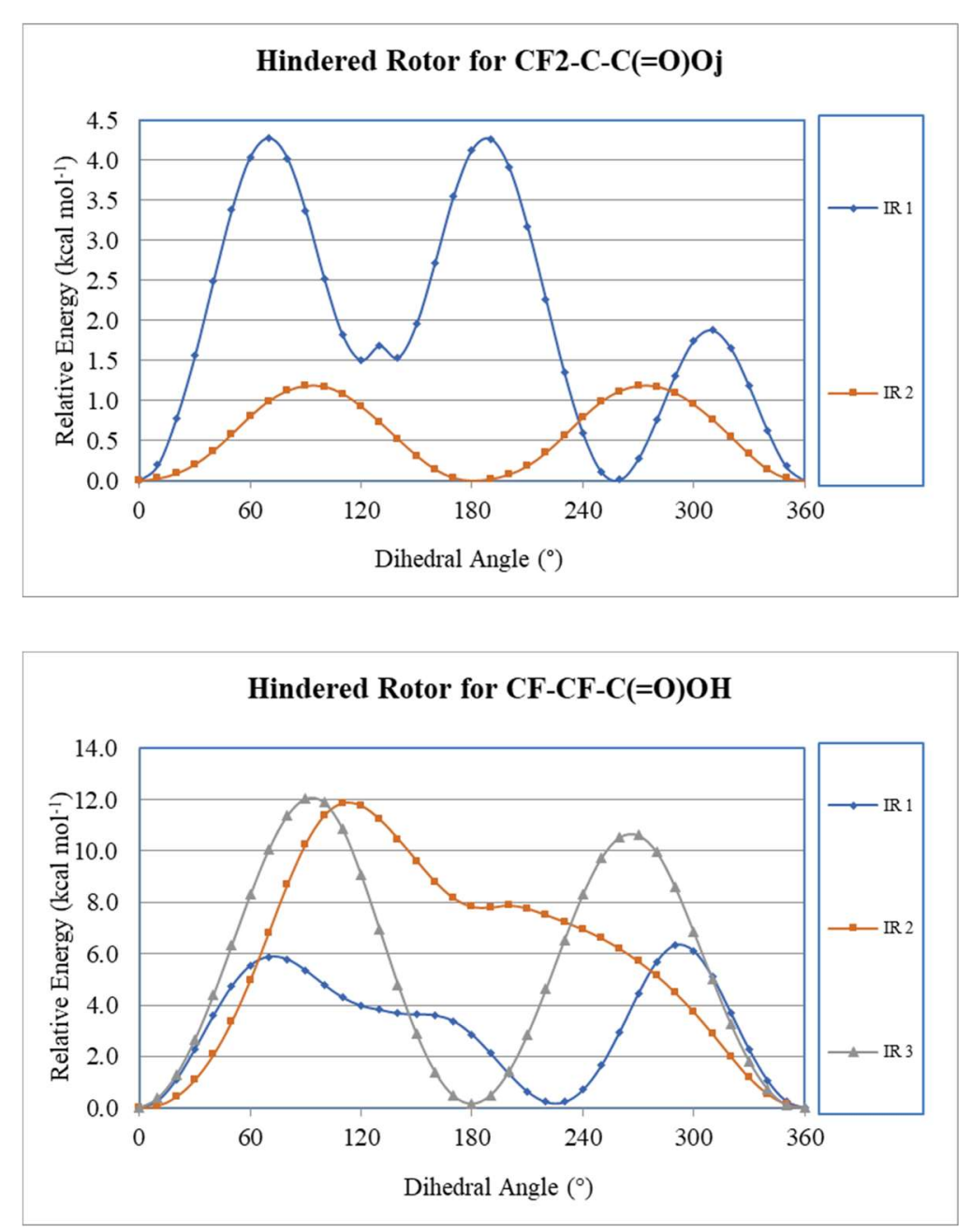
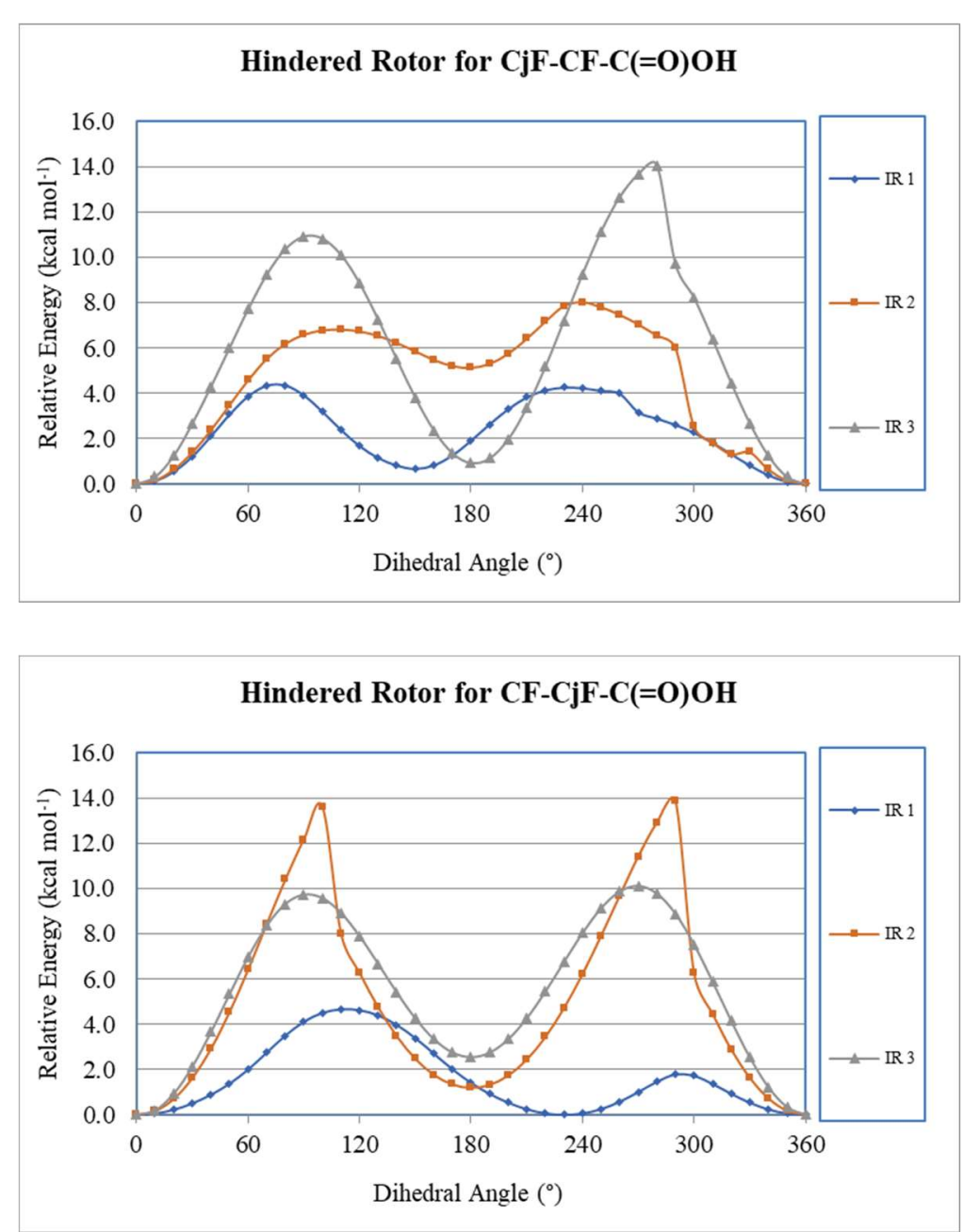

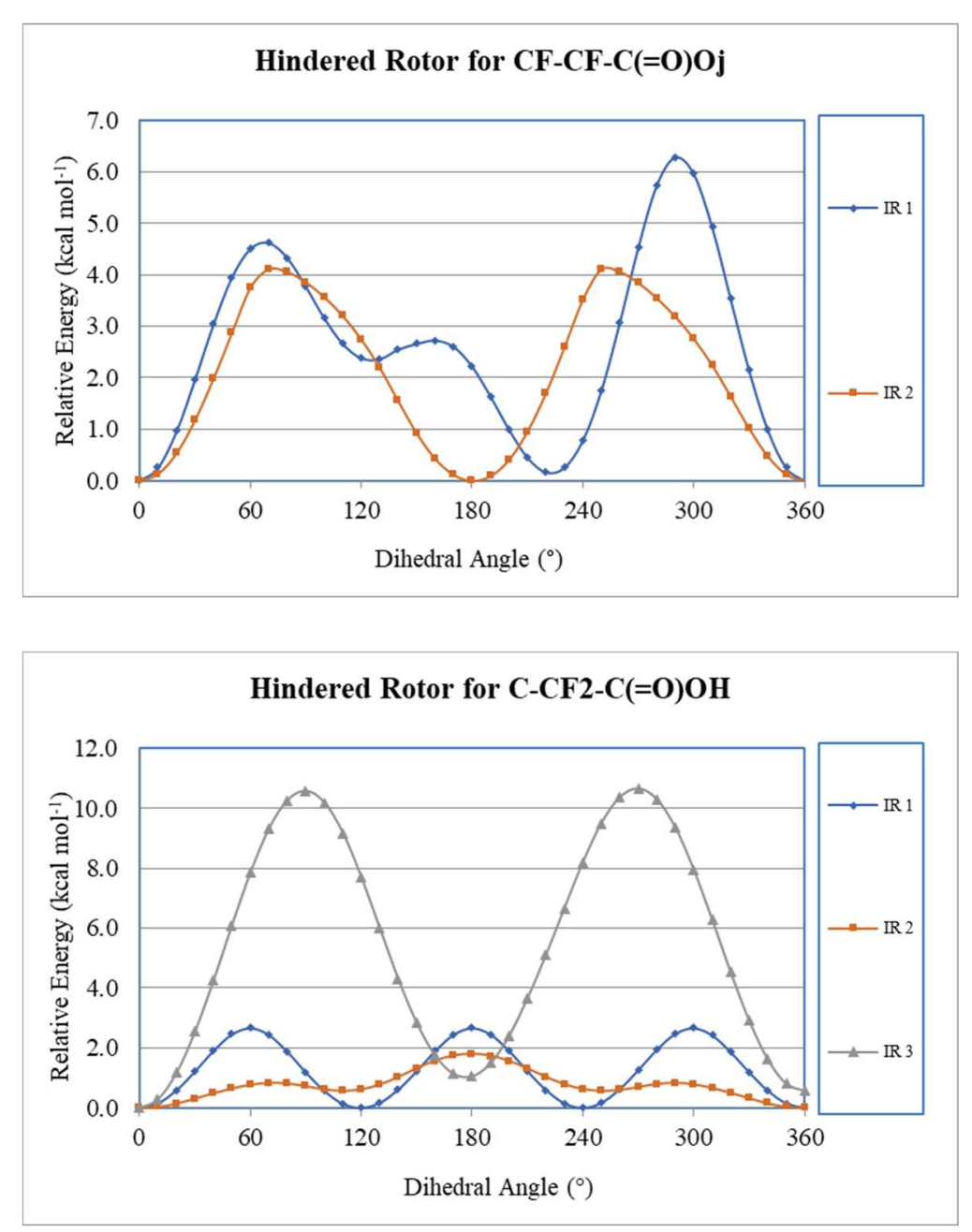
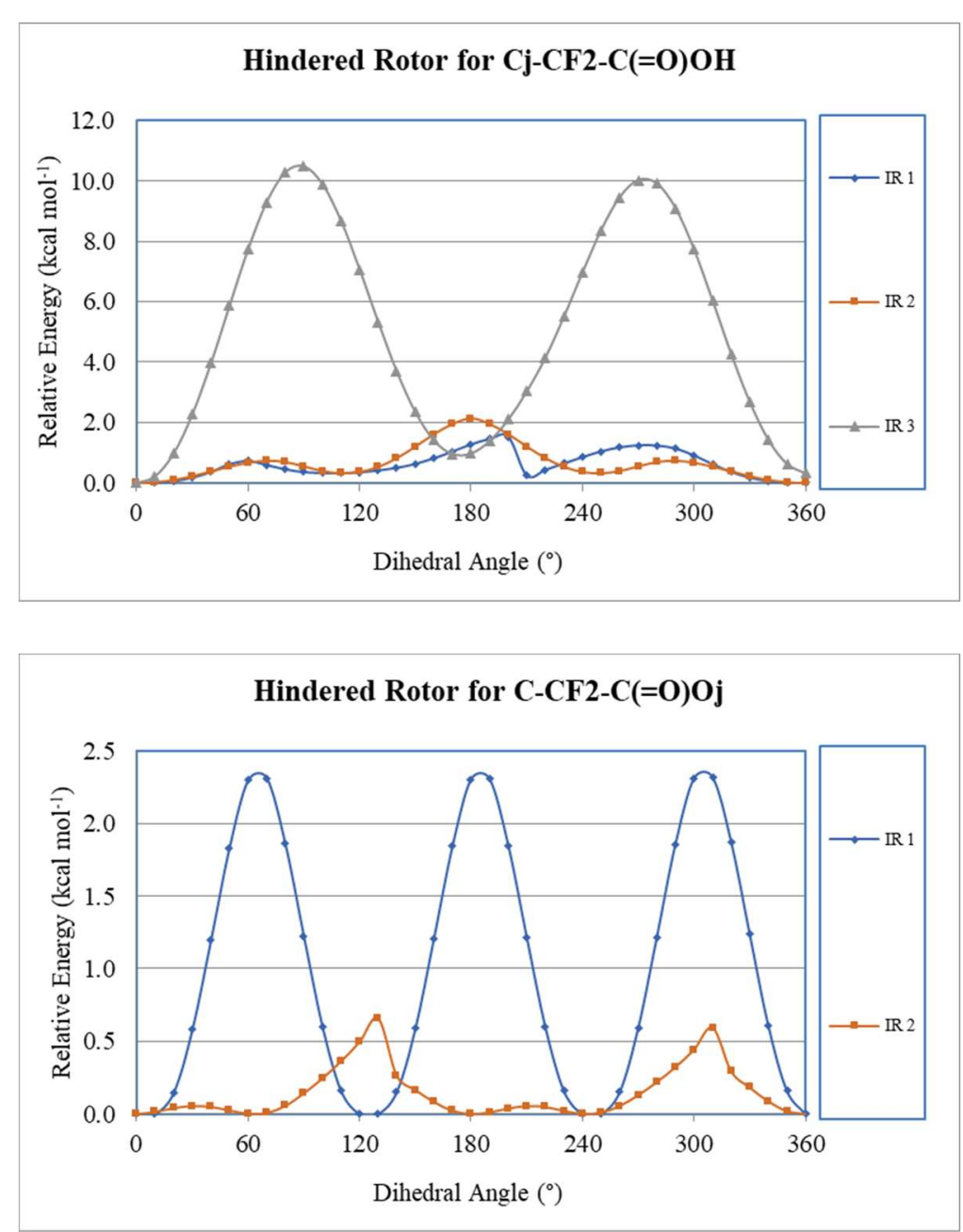

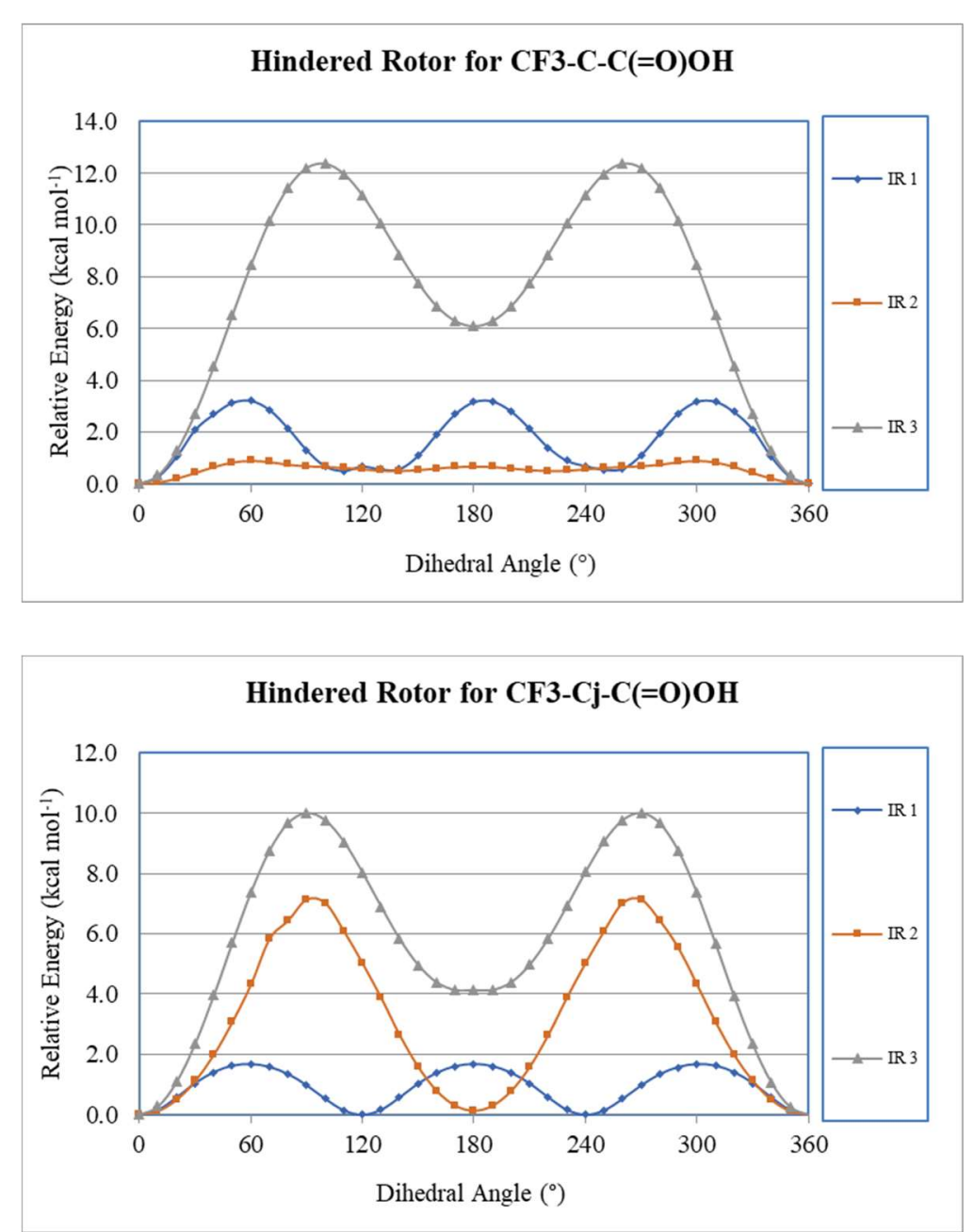
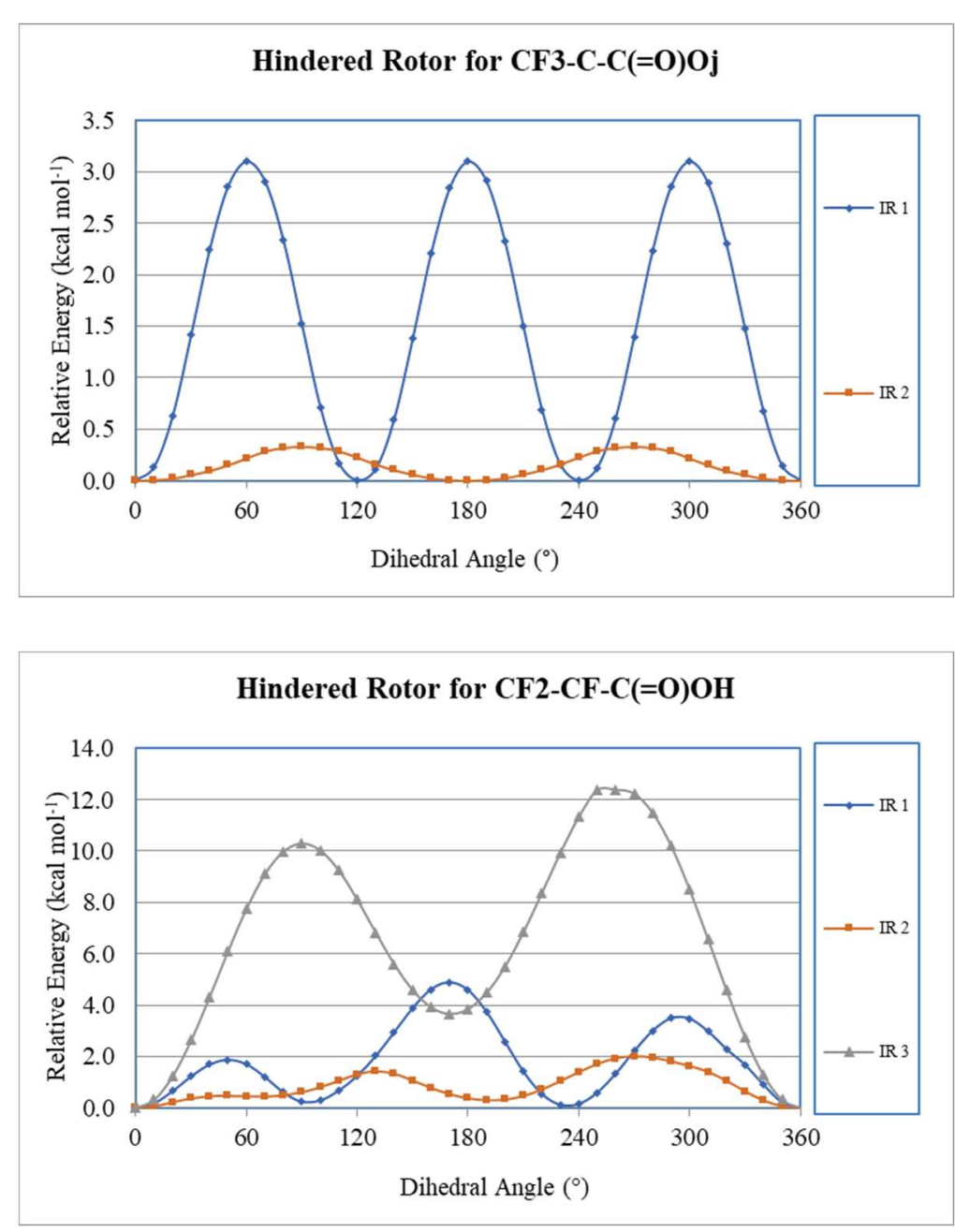

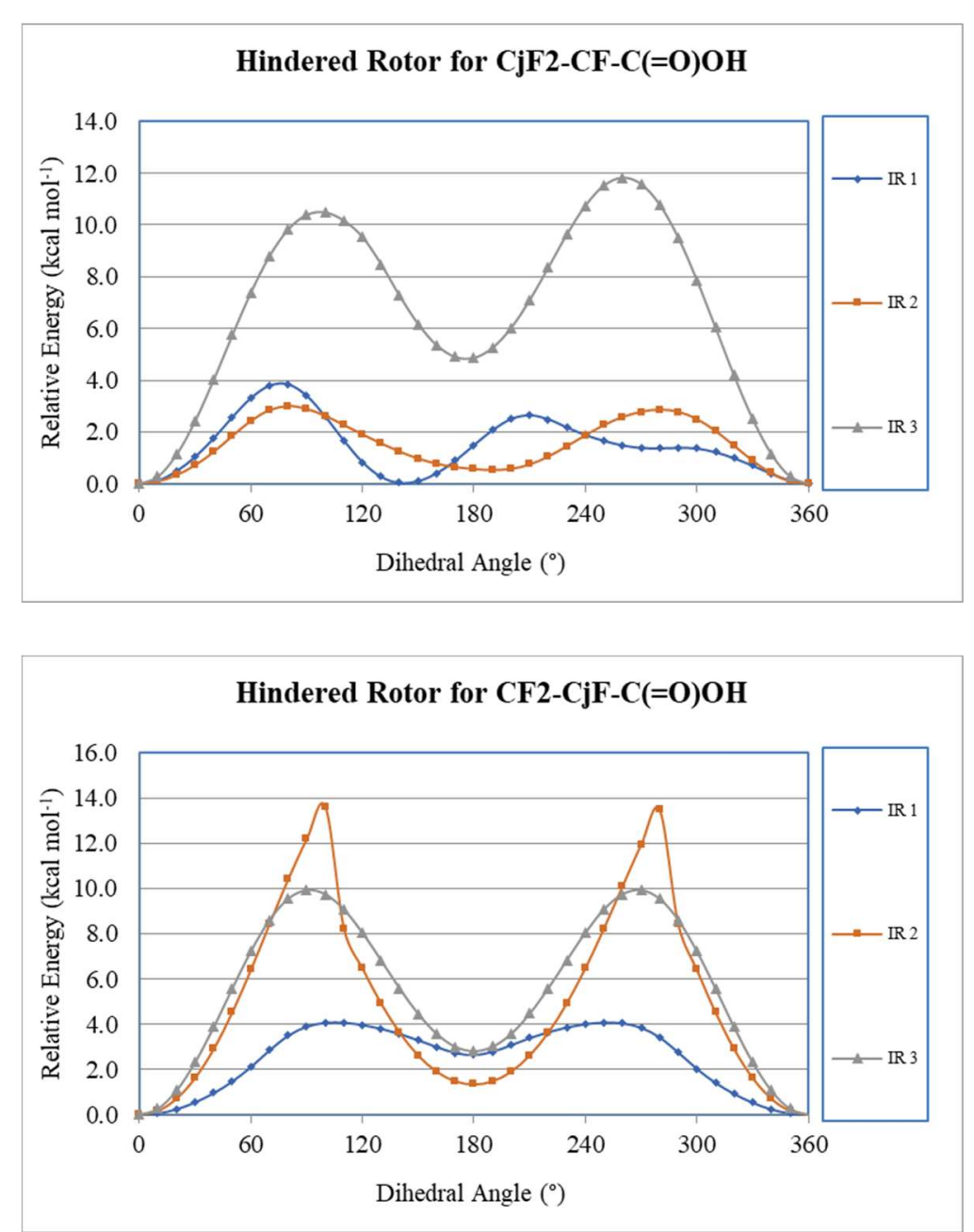
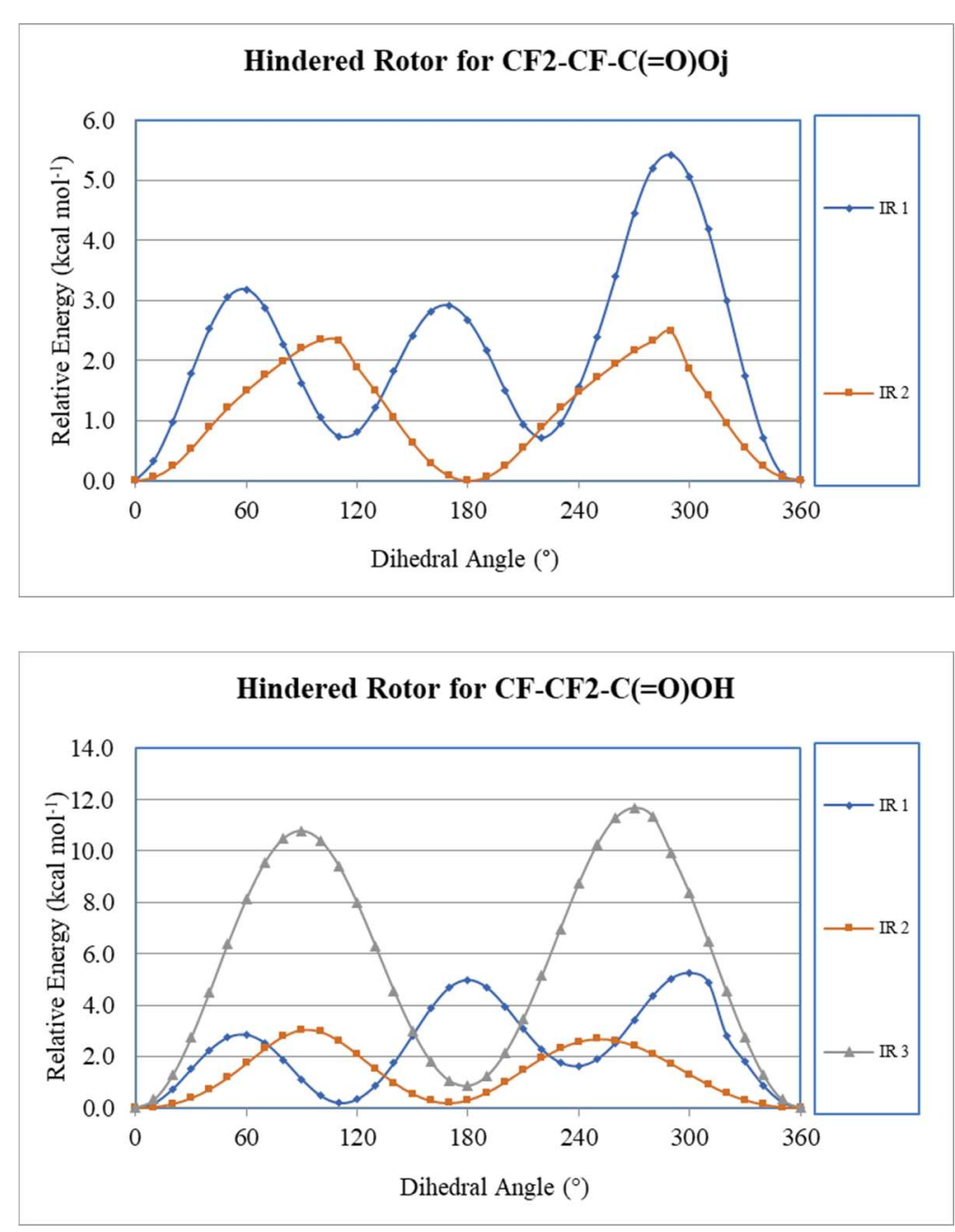

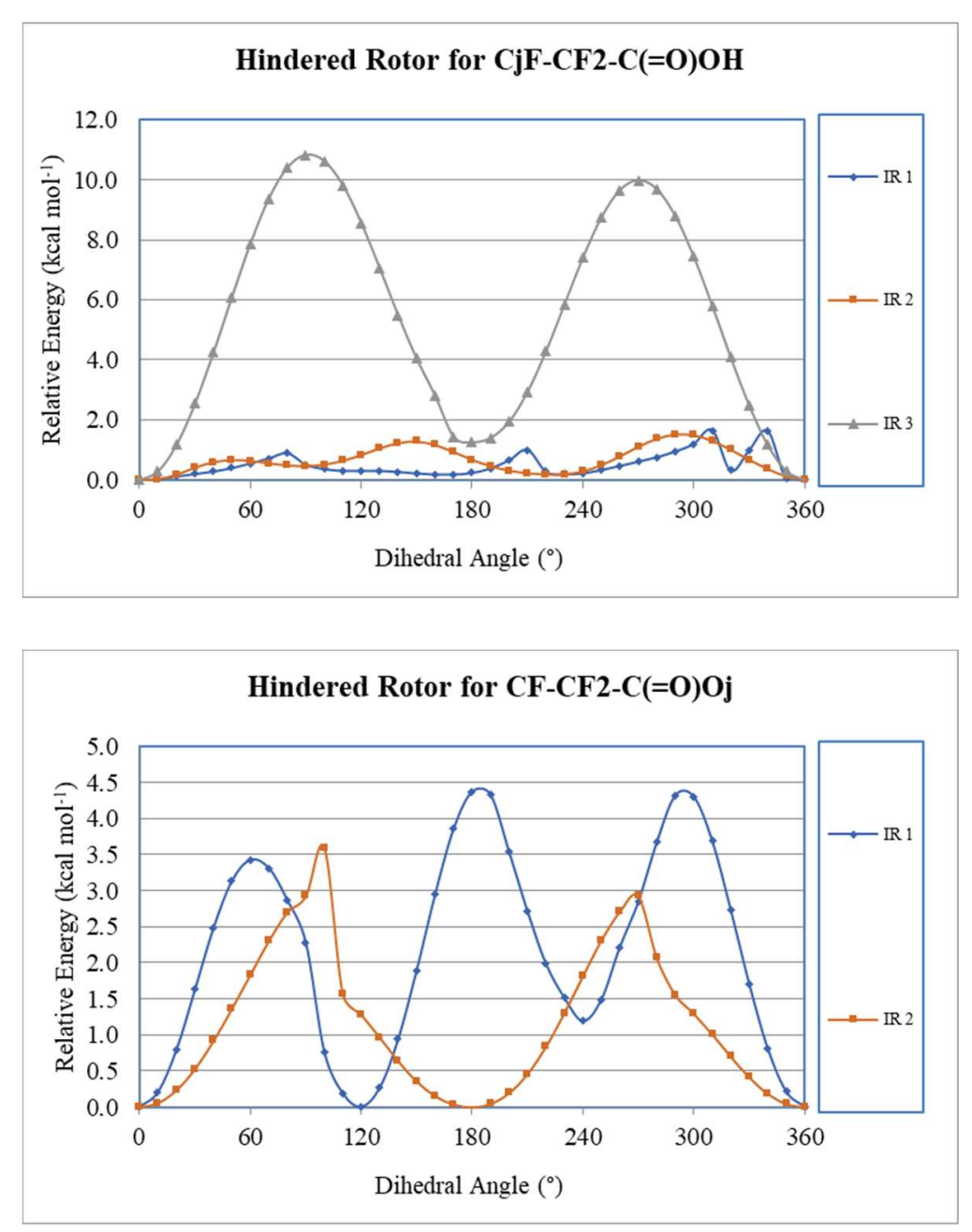
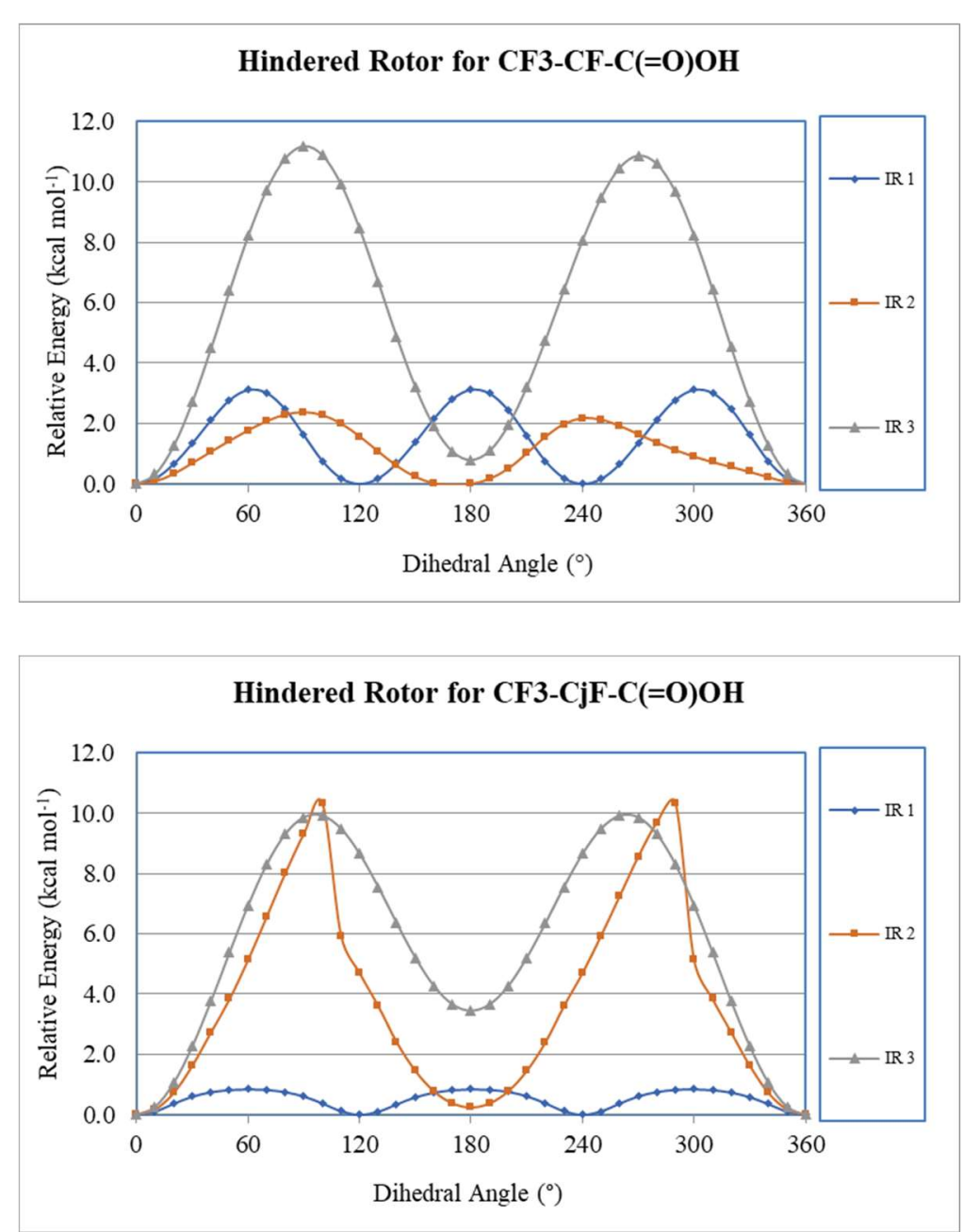

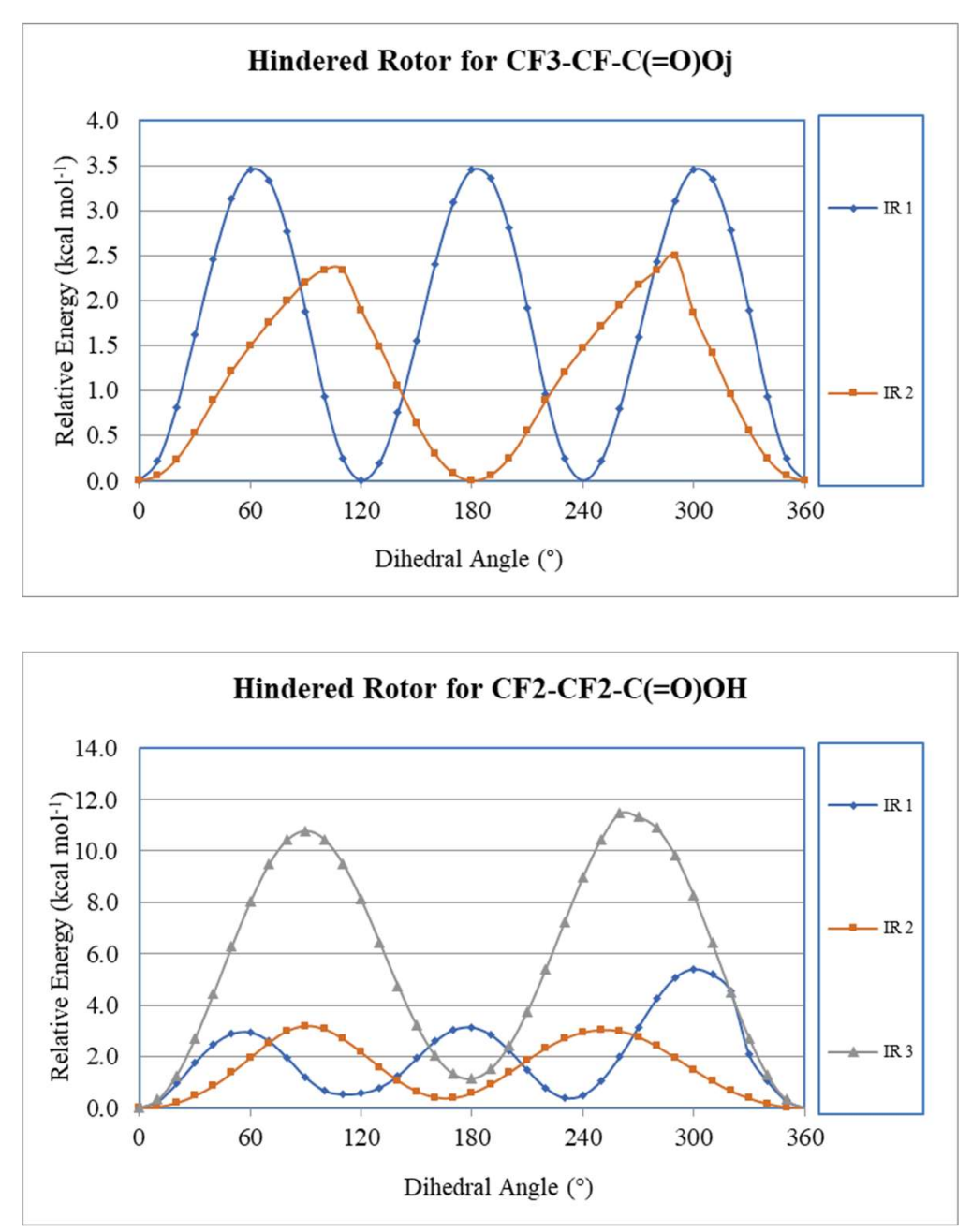
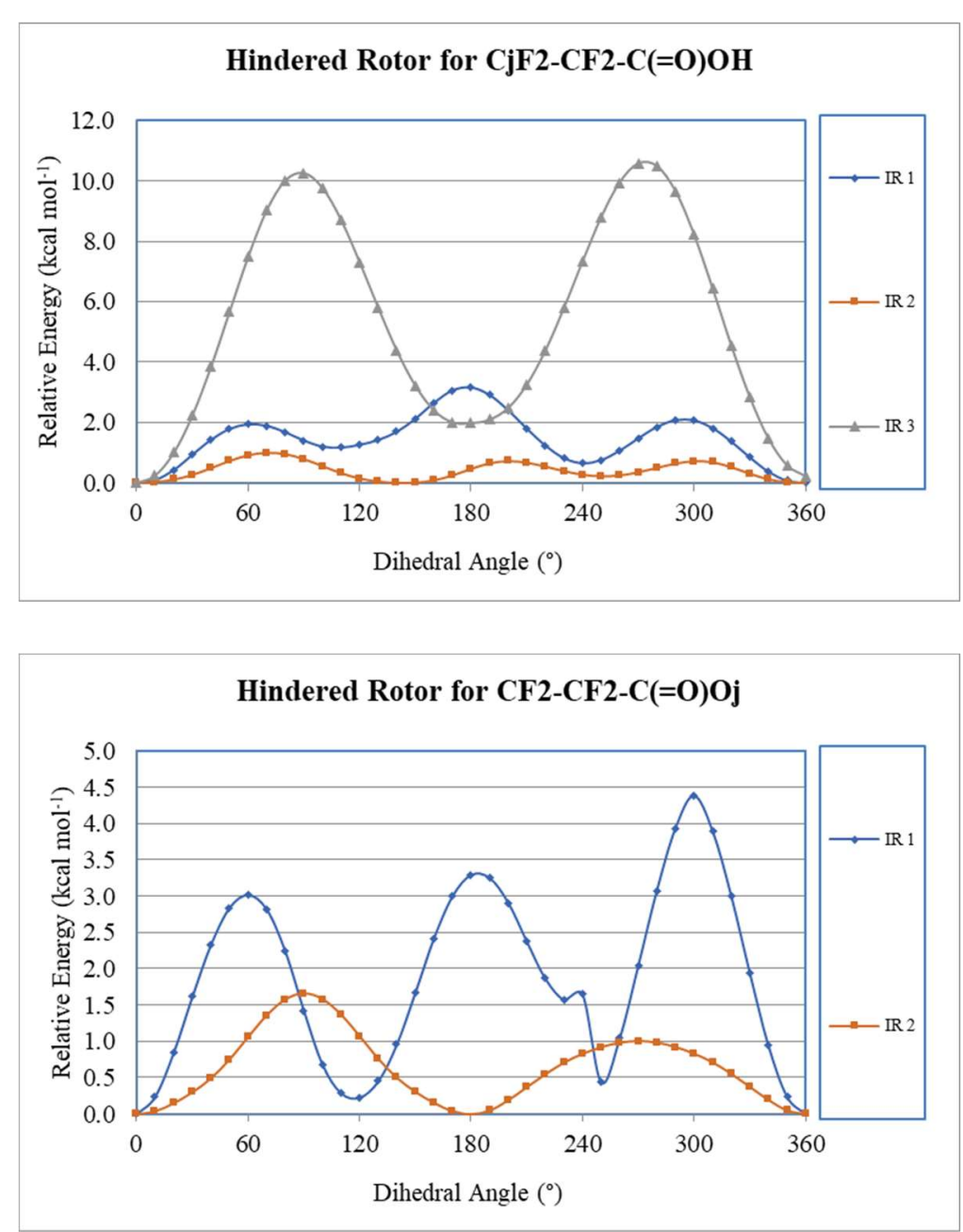

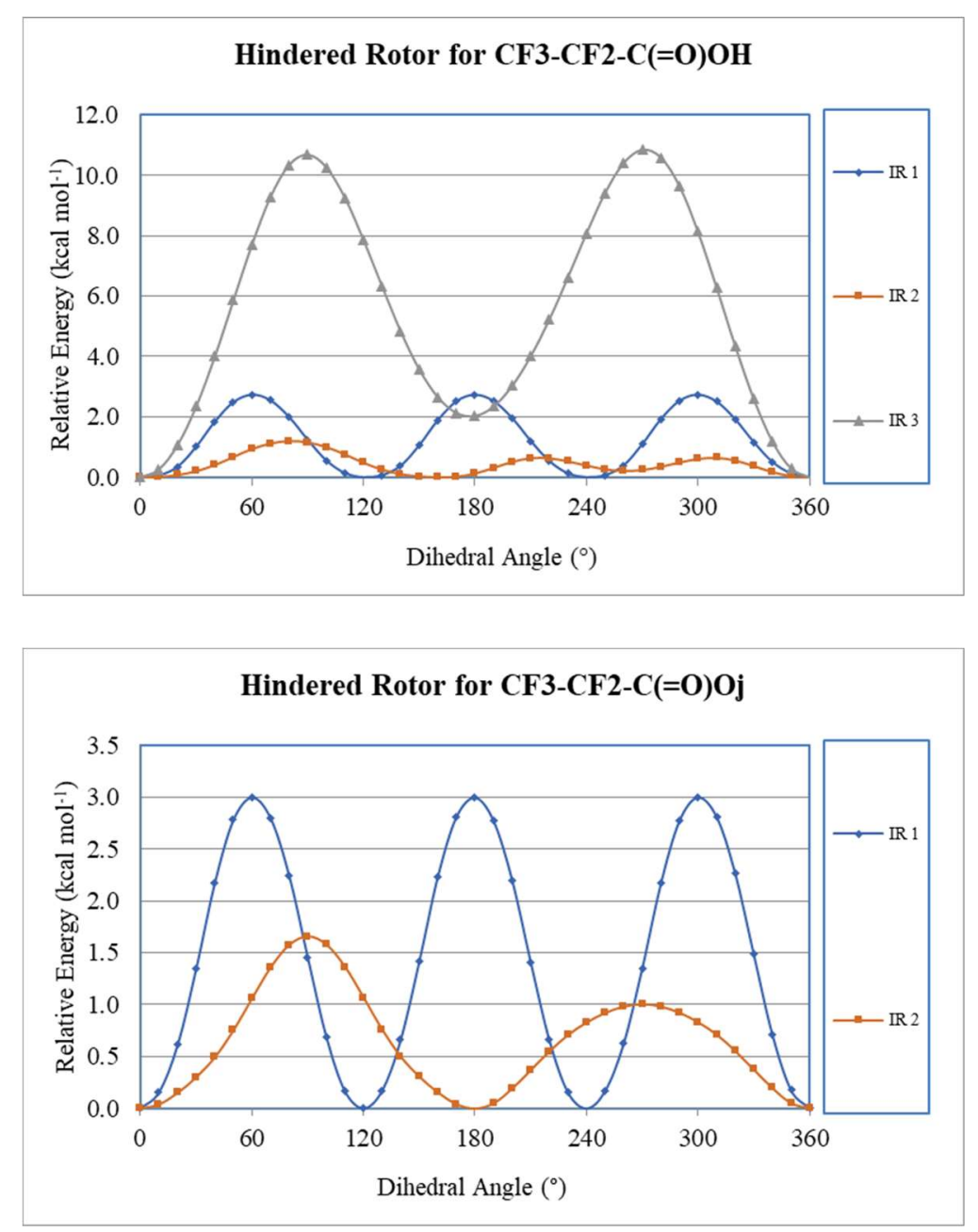
NAME (name of molecule)

$\underline{\text { SMCPs input file for } \mathrm{CH}_{3} \mathrm{CF}_{2} \mathrm{C}(=\mathrm{O}) \mathrm{OH}}$

$\mathrm{C}-\mathrm{CF} 2-\mathrm{COOH}$

COMMENTS:

MN15/cc-pVTZ

\section{TEMPERATURE}

8 (Number of temperature to be read in)

298.1530040050060080010001500

ROTOR

2 number of internal rotors

\section{MOLECULAR WT}

110.01794

\section{OPTICAL ISOMER}

1

Multiplicity

HF298

STOICHIOMETRY (in form of "atom xnumber of atom x")

C 3 H 4 F 2 O 2

rem RSCALING FACTOR (Uses Scott \& Radom's scaling factors) (int) rem 18

USCALING FACTOR (User define scaling factors: ZPE, Hvib, Svib, Cpvib) $0.979,0.979,0.979,0.979$ (include decimal input)

MOMENT (1)=10 e-40 $\mathrm{g}^{*} \mathrm{~cm}^{\wedge} 2(2)=\mathrm{GHz}(3)=\mathrm{amu}-\mathrm{Bohr}^{\wedge} 2(4)=\mathrm{amu}-\mathrm{Angstrom}^{\wedge} 2$

3 choice of moment of inertia units 491.36266726 .72413884 .78977 
SYMMETRY

NON-LINEAR

FREQ (The format for the frequencies is not important. Units are cm-1)

27

$\begin{array}{lll}36.4331 & 219.8028 & 229.7845\end{array}$

$245.2157 \quad 354.1954 \quad 375.1021$

$\begin{array}{lll}418.1880 & 570.5518 & 584.6431\end{array}$

$\begin{array}{lll}607.5127 & 762.8824 & 796.5020\end{array}$

$\begin{array}{lll}943.7380 & 967.4357 & 1165.6830\end{array}$

$\begin{array}{lll}1210.8146 & 1220.9837 & 1271.6067\end{array}$

$\begin{array}{lll}1411.5257 & 1431.7715 & 1458.0666\end{array}$

$\begin{array}{lll}1471.6536 & 1882.1341 & 3102.4413\end{array}$

$\begin{array}{lll}3193.3502 & 3195.7386 & 3812.5974\end{array}$

NAME (name of molecule)

$\mathrm{C}-\mathrm{CjF}-\mathrm{COOH}$

$\underline{\text { SMCPs input file for } \mathrm{CH}_{3} \mathrm{CF} \cdot \mathrm{C}(=\mathrm{O}) \mathrm{OH}}$

COMMENTS:

MN15/cc-pVTZ

TEMPERATURE

8 (Number of temperature to be read in)

298.1530040050060080010001500

\section{ROTOR}

1 number of internal rotors

MOLECULAR WT

91.01953

OPTICAL ISOMER

1 
Multiplicity

2

HF298

STOICHIOMETRY (in form of "atom xnumber of atom x")

C $3 \mathrm{H} 4 \mathrm{~F} 1 \mathrm{O} 2$

rem RSCALING FACTOR (Uses Scott \& Radom's scaling factors) (int) rem 18

USCALING FACTOR (User define scaling factors: ZPE, Hvib, Svib, Cpvib)

$0.979,0.979,0.979,0.979$ (include decimal input)

MOMENT (1)=10 e-40 $\mathrm{g}^{*} \mathrm{~cm}^{\wedge} 2(2)=\mathrm{GHz}(3)=\operatorname{amu}^{-B o h r}{ }^{\wedge} 2(4)=$ amu-Angstrom ${ }^{\wedge} 2$

3 choice of moment of inertia units

$333.70997532 .42342 \quad 854.97742$

\section{SYMMETRY}

NON-LINEAR

FREQ (The format for the frequencies is not important. Units are $\mathrm{cm}-1$ )

24

$\begin{array}{cll}133.5648 & 115.8233 & 239.1755 \\ 280.4935 & 355.7736 & 531.7959 \\ 532.9312 & 542.2074 & 715.9370 \\ 766.7363 & 974.1651 & 997.1683 \\ 1144.8086 & 1250.0407 & 1344.1180 \\ 1415.3999 & 1442.4001 & 1449.3442 \\ 1530.3043 & 1758.8069 & 3051.1297 \\ 3101.9488 & 3191.5961 & 3830.7896\end{array}$


$\underline{\text { List of available literature data }}$

\begin{tabular}{|c|c|c|c|c|c|c|c|c|}
\hline species & $\Delta_{f} \mathbf{H}^{\circ}{ }_{298}\left(\mathrm{kcal} \mathrm{mol}^{-1}\right)$ & Reference & species & $\Delta_{f} \mathbf{H}^{\circ}{ }_{298}\left(\mathrm{kcal} \mathrm{mol}^{-1}\right)$ & Reference & species & $\Delta_{f} \mathbf{H}^{\circ}{ }_{298}\left(\mathrm{kcal} \mathrm{mol}^{-1}\right)$ & Reference \\
\hline \multirow[t]{9}{*}{$\mathrm{CH}_{4}$} & $-17.89 \pm 0.07$ & 1 & \multirow[t]{4}{*}{$\mathrm{CHF}_{3}$} & $-166.60 \pm 0.08$ & 1 & \multirow[t]{4}{*}{$\mathrm{CHF}_{2} \mathrm{CH}_{3}$} & $-120.15 \pm 0.13$ & 2 \\
\hline & $-17.8 \pm 0.07$ & 3 & & $-166.34 \pm 0.10$ & 2 & & $-120.87 \pm 0.30$ & 4 \\
\hline & $-17.8 \pm 0.1$ & 5 & & $-166.71 \pm 0.73$ & 4 & & $-179 \pm 0.79$ & 6 \\
\hline & $-17.89 \pm 0.08$ & 7 & & $-166.09 \pm 0.48$ & 8 & & $-119.7 \pm 1.5$ & 9 \\
\hline & $-17.6 \pm 0.3$ & 10 & \multirow[t]{3}{*}{$\mathrm{CF}_{4}$} & $-223.0 \pm 0.3$ & 1 & \multirow[t]{2}{*}{$\mathrm{CHF}_{2} \mathrm{CHF}_{2}$} & $-209.8 \pm 4.2$ & 11 \\
\hline & $-17.81 \pm 0.01$ & 2 & & $-233.10 \pm 0.06$ & 2 & & $-209.94 \pm 0.76$ & 6 \\
\hline & $-17.90 \pm 0.08$ & 12 & & $-223.15 \pm 0.94$ & 4 & \multirow[t]{3}{*}{$\mathrm{CH}_{2} \mathrm{FCH}_{2} \mathrm{~F}$} & $-107.03 \pm 0.20$ & 2 \\
\hline & $-17.78 \pm 0.10$ & 13 & $\mathrm{CH}_{3} \mathrm{CH}_{3}$ & $-20.0 \pm 0.1$ & 1 & & $-103.7 \pm 2.8$ & 11 \\
\hline & -17.88 & 14 & & $-20.04 \pm 0.07$ & 5 & & $-106.00 \pm 0.76$ & 6 \\
\hline \multirow[t]{7}{*}{$\mathrm{CH}_{3} \mathrm{~F}$} & -56.3 & 1 & & $-20.24 \pm 0.12$ & 7 & \multirow[t]{2}{*}{$\mathrm{CH}_{2} \mathrm{FCHF}_{2}$} & $-159.27 \pm 0.76$ & 6 \\
\hline & $-56.28 \pm 0.06$ & 2 & & $-20.07 \pm 0.03$ & 2 & & $-158.9 \pm 1.0$ & 15 \\
\hline & $-56.30 \pm 0.30$ & 4 & & $-20.03 \pm 0.10$ & 13 & \multirow[t]{2}{*}{$\mathrm{CH}_{2} \mathrm{FCF}_{3}$} & $-219.59 \pm 0.76$ & 6 \\
\hline & -56.9 & 16 & & $-20.1 \pm 1.0$ & 11 & & $-214.1 \pm 2.0$ & 9 \\
\hline & $-56.62 \pm 0.48$ & 8 & & $-20.1 \pm 1.0$ & 17 & \multirow[t]{3}{*}{$\mathrm{CHF}_{2} \mathrm{CF}_{3}$} & -267.79 & 4 \\
\hline & $-57.1 \pm 0.2$ & 18 & & $-20.24 \pm 0.12$ & 19 & & $-265.44 \pm 0.76$ & 6 \\
\hline & $-57.4 \pm 1.2$ & 20 & $\mathrm{CH}_{2} \mathrm{FCH}_{3}$ & $-65.03 \pm 0.09$ & 2 & & $-264.0 \pm 1.1$ & 9 \\
\hline \multirow[t]{4}{*}{$\mathrm{CH}_{2} \mathrm{~F}_{2}$} & $-107.70 \pm 0.04$ & 1 & & $-65.42 \pm 0.25$ & 4 & $\mathrm{CF}_{3} \mathrm{CH}(=\mathrm{O})$ & $-185.4 \pm 0.5$ & 21 \\
\hline & $-107.72 \pm 0.08$ & 2 & & -62.5 & 19 & \multirow[t]{3}{*}{$\mathrm{CH}_{3} \mathrm{CH}_{2} \mathrm{CH}_{3}$} & $-25.2 \pm 0.3$ & 10 \\
\hline & $-108.07 \pm 0.52$ & 4 & & $-66.1 \pm 1.0$ & 22 & & $-25.10 \pm 0.04$ & 2 \\
\hline & $-107.67 \pm 0.48$ & 8 & & $-65.13 \pm 0.76$ & 6 & & $-25.02 \pm 0.13$ & 13 \\
\hline $\mathrm{CF}_{3} \mathrm{OH}$ & $-218.11 \pm 0.55$ & 21 & & -62.9 & 9 & $\mathrm{CH}_{3} \mathrm{OH}$ & $-48.2 \pm 0.3$ & 10 \\
\hline $\mathrm{CH}_{2} \mathrm{FCH}_{2} \mathrm{O} \bullet$ & -45 & 16 & $\mathrm{CF}_{3} \mathrm{CH}_{3}$ & $-180.51 \pm 0.51$ & 4 & & $-48.02 \pm 0.04$ & 2 \\
\hline \multirow[t]{2}{*}{$\mathrm{CH}_{2} \cdot \mathrm{CH}_{2} \mathrm{OH}$} & $-5.60 \pm 0.20$ & 2 & & $-179 \pm 0.79$ & 6 & & $-48.16 \pm 0.07$ & 4 \\
\hline & $-5.7 \pm 0.85$ & 23 & & $-178.2 \pm 0.4$ & 9 & & $-48.08 \pm 0.05$ & 19 \\
\hline
\end{tabular}

S29 


\begin{tabular}{|c|c|c|c|c|c|c|c|c|}
\hline species & $\Delta_{f} \mathrm{H}^{\circ}{ }_{298}\left(\mathrm{kcal} \mathrm{mol}^{-1}\right)$ & Reference & species & $\Delta_{f} \mathrm{H}^{\circ}{ }_{298}\left(\mathrm{kcal} \mathrm{mol}^{-1}\right)$ & Reference & species & $\Delta_{f} \mathrm{H}^{\circ}{ }_{298}\left(\mathrm{kcal} \mathrm{mol}^{-1}\right)$ & Reference \\
\hline \multirow[t]{4}{*}{$\mathrm{CF}_{3} \mathrm{CF}_{3}$} & $-320.86 \pm 0.24$ & 2 & $\mathrm{CH}_{3} \mathrm{O} \bullet$ & $4.9 \pm 0.4$ & 10 & $\mathrm{CH}_{3} \mathrm{CH}_{2} \mathrm{O} \bullet$ & $-2.9 \pm 0.1$ & 10 \\
\hline & $-322.90 \pm 0.96$ & 6 & & $5.13 \pm 0.07$ & 2 & & $-3.01 \pm 0.11$ & 2 \\
\hline & $-320.9 \pm 1.5$ & 9 & & $5.14 \pm 0.08$ & 24 & $\mathrm{CHF}_{2} \mathrm{CH}_{2} \mathrm{O} \cdot$ & -98.1 & 16 \\
\hline & $-320.9 \pm 1.5$ & 25 & $\mathrm{CH}_{3} \mathrm{CH}_{2} \mathrm{CH}_{2} \mathrm{CH}_{3}$ & $-30.0 \pm 0.1$ & 10 & $\mathrm{H} \bullet$ & $52.10 \pm 0.00$ & 2 \\
\hline \multirow[t]{2}{*}{$\mathrm{CF}_{3} \mathrm{CHF} \bullet$} & -166.1 & 16 & & $-30.06 \pm 0.06$ & 2 & & $52.1 \pm 0.0$ & 26 \\
\hline & $-166.1 \pm 0.9$ & 27 & $\mathrm{CH}_{3} \mathrm{C}(=\mathrm{O}) \mathrm{CH}_{3}$ & $-51.9 \pm 0.2$ & 10 & $\mathrm{~F} \bullet$ & $18.97 \pm 0.01$ & 2 \\
\hline \multirow[t]{2}{*}{$\mathrm{CH}_{2} \mathrm{FCH}_{2} \mathrm{CH}_{3}$} & $-70.24 \pm 0.21$ & 4 & & $-51.81 \pm 0.09$ & 2 & & $18.90 \pm 0.07$ & 26 \\
\hline & $-70.24 \pm 1.30$ & 28 & & $-51.9 \pm 0.2$ & 13 & $\mathrm{CH}_{3} \bullet$ & $35.00 \pm 0.01$ & 2 \\
\hline $\mathrm{CH}_{2} \mathrm{FOH}$ & $-101.94 \pm 0.29$ & 29 & $\mathrm{CH}_{3} \mathrm{CH}_{2} \mathrm{CH}_{2} \mathrm{CH}_{2} \mathrm{~F}$ & $-75.17 \pm 0.21$ & 4 & & $35.20 \pm 0.20$ & 21 \\
\hline $\mathrm{CHF}_{2} \mathrm{OH}$ & $-161.59 \pm 0.45$ & 29 & $\mathrm{CH}_{3} \mathrm{CH}_{2} \mathrm{CHF}_{2}$ & $-125.82 \pm 0.47$ & 4 & $\mathrm{CH}_{2} \mathrm{~F} \bullet$ & -7.7 & 16 \\
\hline \multirow[t]{4}{*}{$\mathrm{CH}_{3} \mathrm{CH}(=\mathrm{O})$} & $-39.60 \pm 0.06$ & 2 & $\mathrm{CH}_{3} \mathrm{CF}_{2} \mathrm{CH}_{3}$ & $-133.25 \pm 0.34$ & 4 & & $-7.46 \pm 0.38$ & 8 \\
\hline & $-39.70 \pm 0.12$ & 13 & $\mathrm{CHF}_{2} \mathrm{C}(=\mathrm{O}) \mathrm{CH}_{3}$ & $-144.5 \pm 0.4$ & 10 & $\mathrm{CF}_{3} \mathrm{CH}_{2} \bullet$ & $-125.7 \pm 0.7$ & 28 \\
\hline & $-39.7 \pm 0.1$ & 21 & $\mathrm{CHF}_{2} \mathrm{CHFCH}(=\mathrm{O})$ & $-181.8 \pm 0.8$ & 1 & $\mathrm{CHF}_{2} \bullet$ & -58.6 & 16 \\
\hline & $-40.80 \pm 0.35$ & 30 & $\mathrm{CHF}_{2} \mathrm{CF}(=\mathrm{O})$ & $-190.1 \pm 1.0$ & 21 & & $-58.08 \pm 0.86$ & 8 \\
\hline $\mathrm{CH}_{2} \mathrm{FCH}(=\mathrm{O})$ & $-80.4 \pm 0.7$ & 21 & $\mathrm{CH}_{2} \mathrm{FC}(=\mathrm{O}) \mathrm{CH}_{3}$ & $-94.3 \pm 1.4$ & 2 & $\mathrm{CF}_{3} \bullet$ & $-111.80 \pm 0.11$ & 2 \\
\hline $\mathrm{CHF}_{2} \mathrm{CH}(=\mathrm{O})$ & $-130.0 \pm 0.6$ & 21 & $\mathrm{CF}_{3} \mathrm{CF}(=\mathrm{O})$ & $-245.8 \pm 1.4$ & 21 & & -112.2 & 16 \\
\hline \multirow[t]{3}{*}{$\mathrm{CH}_{3} \mathrm{CH}_{2} \mathrm{OH}$} & $-56.17 \pm 0.05$ & 2 & $\mathrm{CF}_{3} \mathrm{C}(=\mathrm{O}) \mathrm{CH}_{2} \mathrm{~F}$ & $-237.2 \pm 1.6$ & 10 & $\mathrm{CH}_{3} \mathrm{CH}_{2} \bullet$ & $28.9 \pm 0.4$ & 1 \\
\hline & $-56.12 \pm 0.2$ & 19 & $\mathrm{CHF}_{2} \mathrm{C}(=\mathrm{O}) \mathrm{CHF}_{2}$ & $-230.0 \pm 0.8$ & 10 & & $28.68 \pm 0.06$ & 2 \\
\hline & $-56.07 \pm 0.05$ & 25 & $\mathrm{CHF}_{2} \mathrm{CF}_{2} \mathrm{CF}(=\mathrm{O})$ & $-289.6 \pm 1.1$ & 21 & & $28.9 \pm 0.4$ & 21 \\
\hline $\mathrm{CH}_{2} \cdot \mathrm{CH}(=\mathrm{O})$ & $4.40 \pm 0.90$ & 10 & $\mathrm{CH}_{2} \mathrm{FC}(=\mathrm{O}) \mathrm{CH}_{2} \mathrm{~F}$ & $-133.2 \pm 1.5$ & 10 & & $28.80 \pm 0.50$ & 31 \\
\hline \multirow[t]{4}{*}{$\mathrm{CH}_{3} \mathrm{CH}_{2} \mathrm{CH}(=\mathrm{O})$} & $-44.36 \pm 0.19$ & 10 & $\mathrm{CF}_{3} \mathrm{CF}_{2} \mathrm{CH}(=\mathrm{O})$ & $-285.2 \pm 2.6$ & 21 & $\mathrm{CH}_{3} \mathrm{CHF} \bullet$ & $-17.8 \pm 0.7$ & 27 \\
\hline & $-44.64 \pm 0.06$ & 2 & $\mathrm{CF}_{3} \mathrm{C}(=\mathrm{O}) \mathrm{CHF}_{2}$ & $-285.5 \pm 1.0$ & 10 & $\mathrm{CH}_{3} \mathrm{CF}_{2} \bullet$ & $-71.1 \pm 0.7$ & 27 \\
\hline & $-44.36 \pm 0.19$ & 13 & $\mathrm{CF}_{3} \mathrm{CHFCF}(=\mathrm{O})$ & $-298.6 \pm 0.6$ & 21 & $\mathrm{CH}_{2} \mathrm{FCH}_{2} \bullet$ & $-14.3 \pm 0.7$ & 27 \\
\hline & $-44.4 \pm 0.2$ & 27 & $\mathrm{CH}_{2} \mathrm{FO}$ & $-49.11 \pm 0.37$ & 29 & $\mathrm{CH}_{2} \mathrm{FCHF} \bullet$ & $-59.3 \pm 0.7$ & 27 \\
\hline $\mathrm{CH}_{2} \mathrm{FCH}_{2} \mathrm{OH}$ & -101.7 & 16 & $\mathrm{CH}_{3} \mathrm{C} \cdot(=\mathrm{O})$ & $-2.4 \pm 0.1$ & 10 & $\mathrm{CH}_{2} \mathrm{FCF}_{2} \bullet$ & -110.3 & 16 \\
\hline $\mathrm{CHF} \bullet \mathrm{CH}_{2} \mathrm{OH}$ & -50.7 & 16 & & $-2.39 \pm 0.08$ & 2 & & $-109.97 \pm 1.39$ & 11 \\
\hline \multirow[t]{2}{*}{$\mathrm{CH}_{2} \bullet \mathrm{OH}$} & $-4.00 \pm 0.07$ & 2 & $\mathrm{CF}_{2} \bullet \mathrm{CH}(=\mathrm{O})$ & $-92.6 \pm 2.5$ & 10 & $\mathrm{CHF}_{2} \mathrm{CHF} \bullet$ & -110.8 & 16 \\
\hline & $-3.96 \pm 0.08$ & 28 & $\mathrm{CHF}_{2} \mathrm{O} \bullet$ & $-97.82 \pm 0.48$ & 29 & & $-109.89 \pm 1.48$ & 11 \\
\hline
\end{tabular}




\begin{tabular}{|c|c|c|c|c|c|c|c|c|}
\hline species & $\Delta_{f} \mathbf{H}^{\circ}{ }_{298}\left(\mathrm{kcal} \mathrm{mol}^{-1}\right)$ & Reference & species & $\Delta_{f} \mathbf{H}^{\circ}{ }_{298}\left(\mathrm{kcal} \mathrm{mol}^{-1}\right)$ & Reference & species & $\Delta_{f} \mathbf{H}^{\circ}{ }_{298}\left(\mathrm{kcal} \mathrm{mol}^{-1}\right)$ & Reference \\
\hline $\mathrm{CHF}_{2} \mathrm{CH}_{2} \bullet$ & $-67.4 \pm 0.7$ & 27 & $\mathrm{CHF} \cdot \mathrm{CH}(=\mathrm{O})$ & $-45.3 \pm 1.6$ & 21 & $\mathrm{CH}_{2} \mathrm{FCH}_{2} \mathrm{CH}(=\mathrm{O})$ & $-87.4 \pm 1.2$ & 21 \\
\hline \multirow[t]{2}{*}{$\mathrm{CHF}_{2} \mathrm{CF}_{2} \bullet$} & -160.2 & 1 & $\mathrm{CHF}_{2} \mathrm{CH}_{2} \mathrm{OH}$ & -154.1 & 16 & $\mathrm{CF}_{3} \mathrm{CHFCH}(=\mathrm{O})$ & $-237.5 \pm 0.8$ & 21 \\
\hline & $-160.40 \pm 1.53$ & 11 & $\mathrm{CH}_{3} \mathrm{CF}(=\mathrm{O})$ & $-105.8 \pm 0.7$ & 21 & $\mathrm{CF}_{3} \mathrm{CH}_{2} \mathrm{CF}(=\mathrm{O})$ & $-262.5 \pm 0.6$ & 21 \\
\hline \multirow[t]{3}{*}{$\mathrm{CF}_{3} \mathrm{CF}_{2} \bullet$} & -213.8 & 16 & $\mathrm{CH}_{2} \mathrm{FCF}(=\mathrm{O})$ & $-143.6 \pm 0.7$ & 21 & $\mathrm{CHF}_{2} \mathrm{C}(=\mathrm{O}) \mathrm{CH}_{2} \mathrm{~F}$ & $-182.0 \pm 0.5$ & 10 \\
\hline & $-213.77 \pm 0.67$ & 8 & $\mathrm{CHF}_{2} \mathrm{CH}_{2} \mathrm{CH}(=\mathrm{O})$ & $-141.1 \pm 0.6$ & 21 & $\mathrm{CH}_{2} \bullet \mathrm{CF}(=\mathrm{O})$ & $-60.1 \pm 0.7$ & 21 \\
\hline & $-213.8 \pm 0.7$ & 27 & $\mathrm{CF}_{3} \mathrm{C}(=\mathrm{O}) \mathrm{CH}_{3}$ & $-200.0 \pm 0.4$ & 10 & $\mathrm{CH}_{2} \mathrm{FC} \bullet(=\mathrm{O})$ & $-40.9 \pm 2.2$ & 21 \\
\hline \multirow[t]{2}{*}{$\mathrm{CH}_{2} \mathrm{FC}(=\mathrm{O}) \mathrm{CH}_{2} \bullet$} & $-50.3 \pm 1.9$ & 10 & $\mathrm{CF}_{3} \mathrm{CH}_{2} \mathrm{CH}(=\mathrm{O})$ & $-198.9 \pm 0.5$ & 21 & $\mathrm{CH}_{2} \mathrm{FC}(=\mathrm{O}) \mathrm{CHF} \bullet$ & $-97.2 \pm 1.6$ & 10 \\
\hline & & & $\mathrm{CH}_{3} \mathrm{CH}_{2} \mathrm{CF}_{3}$ & $-185.48 \pm 0.56$ & 4 & $\mathrm{CHF}_{2} \mathrm{C}(=\mathrm{O}) \mathrm{CH}_{2} \bullet$ & $-100.5 \pm 2.8$ & 10 \\
\hline
\end{tabular}


Uncertainty at $95 \%$ confidence limit

The enthalpy of reaction from calculations compared with experimental enthalpy of reaction for reference species

\begin{tabular}{|c|c|c|c|c|c|c|c|c|c|c|c|c|c|}
\hline \multirow{2}{*}{\multicolumn{7}{|c|}{ Reaction }} & \multicolumn{7}{|c|}{$\Delta \mathbf{H}_{\mathrm{rxn}}\left(\mathrm{kcal} \mathrm{mol}^{-1}\right)$} \\
\hline & & & & & & & \multirow{2}{*}{$\begin{array}{r}\text { experimental } \\
-104.92\end{array}$} & \multirow{2}{*}{$\begin{array}{r}\text { M062X } \\
-104.57\end{array}$} & \multirow{2}{*}{$\begin{array}{l}\text { B3LYP } \\
-104.24\end{array}$} & \multirow{2}{*}{$\frac{\text { MN15 }}{-104.23}$} & \multirow{2}{*}{$\begin{array}{r}\text { CBS-QB3 } \\
-105.39\end{array}$} & \multirow{2}{*}{$\begin{array}{r}\text { CBS-APNO } \\
-105.43\end{array}$} & \multirow{2}{*}{$\frac{\text { G4 }}{-104.49}$} \\
\hline $\mathrm{CH}_{3} \bullet$ & + & $\mathrm{H} \bullet$ & $\rightarrow$ & $\mathrm{CH}_{4}$ & & & & & & & & & \\
\hline $\mathrm{CH}_{3} \mathrm{CH}_{2} \bullet$ & + & $\mathrm{H} \bullet$ & $\rightarrow$ & $\mathrm{CH}_{3} \mathrm{CH}_{3}$ & & & -100.87 & -100.88 & -99.63 & -99.65 & -101.69 & -101.79 & -100.68 \\
\hline $\mathrm{CH}_{3} \bullet$ & + & $\mathrm{CH}_{3} \mathrm{CH}_{2} \bullet$ & $\rightarrow$ & $\mathrm{CH}_{3} \mathrm{CH}_{2} \mathrm{CH}_{3}$ & & & -88.79 & -89.39 & -82.18 & -88.50 & -89.71 & -89.73 & -87.98 \\
\hline $\mathrm{CH}_{3} \bullet$ & + & $\mathrm{F} \bullet$ & $\rightarrow$ & $\mathrm{CH}_{3} \mathrm{~F}$ & & & -110.25 & -109.59 & -106.88 & -113.21 & -111.98 & -110.84 & -109.85 \\
\hline $\mathrm{CH}_{3} \bullet$ & + & $\mathrm{CH}_{4}$ & $\rightarrow$ & $\mathrm{CH}_{3} \mathrm{CH}_{3}$ & + & $\mathrm{H} \bullet$ & 14.83 & 13.56 & 19.16 & 13.64 & 14.67 & 14.77 & 15.48 \\
\hline $\mathrm{CH}_{3} \bullet$ & + & $\mathrm{CH}_{3} \mathrm{CH}_{2} \mathrm{CH}_{3}$ & $\rightarrow$ & $\mathrm{CH}_{3} \mathrm{CH}_{2} \mathrm{CH}_{2} \mathrm{CH}_{3}$ & + & $\mathrm{H} \bullet$ & 12.16 & 11.05 & 17.50 & 11.13 & 11.85 & 11.98 & 12.52 \\
\hline $\mathrm{CH}_{3} \cdot$ & + & $\mathrm{CH}_{3} \mathrm{CH}_{2} \mathrm{CH}_{3}$ & $\rightarrow$ & $\mathrm{CH}_{3} \mathrm{CH}_{2} \bullet$ & + & $\mathrm{CH}_{3} \mathrm{CH}_{3}$ & -1.30 & -1.62 & -2.90 & -2.09 & -1.01 & -0.93 & -1.04 \\
\hline $\mathrm{CH}_{3} \cdot$ & + & $\mathrm{CH}_{2} \mathrm{FCHF}_{2}$ & $\rightarrow$ & $\mathrm{CHF}_{2} \mathrm{CH}_{3}$ & + & $\mathrm{CH}_{2} \mathrm{~F} \bullet$ & -3.35 & -3.47 & -4.25 & -4.77 & -3.17 & -3.95 & -3.26 \\
\hline $\mathrm{CH}_{3} \cdot$ & + & $\mathrm{CH}_{2} \mathrm{FCHF}_{2}$ & $\rightarrow$ & $\mathrm{CH}_{2} \mathrm{FCF}_{2} \bullet$ & + & $\mathrm{CH}_{4}$ & -3.52 & -3.15 & -4.77 & -5.48 & -2.74 & -3.51 & -3.87 \\
\hline $\mathrm{CH}_{3} \bullet$ & + & $\mathrm{CH}_{2} \mathrm{FCHF}_{2}$ & $\rightarrow$ & $\mathrm{CHF}_{2} \mathrm{CHF} \cdot$ & + & $\mathrm{CH}_{4}$ & -3.44 & -3.59 & -4.95 & -5.45 & -2.99 & -3.43 & -4.09 \\
\hline $\mathrm{CH}_{3} \cdot$ & + & $\mathrm{CH}_{2} \mathrm{FCH}_{3}$ & $\rightarrow$ & $\mathrm{CH}_{3} \mathrm{CH}_{3}$ & + & $\mathrm{CH}_{2} \mathrm{~F} \bullet$ & 2.49 & 2.25 & 1.85 & 0.47 & 2.40 & 1.64 & 2.23 \\
\hline $\mathrm{CH}_{3} \cdot$ & + & $\mathrm{CH}_{2} \mathrm{FCH}_{3}$ & $\rightarrow$ & $\mathrm{CH}_{3} \mathrm{CH}_{2} \mathrm{CH}_{3}$ & + & $\mathrm{F} \cdot$ & 23.89 & 22.82 & 26.56 & 26.83 & 25.04 & 23.89 & 24.45 \\
\hline $\mathrm{CH}_{3} \cdot$ & + & $\mathrm{CH}_{2} \mathrm{FCH}_{3}$ & $\rightarrow$ & $\mathrm{CH}_{2} \mathrm{FCH}_{2} \mathrm{CH}_{3}$ & + & $\mathrm{H} \bullet$ & 11.90 & 11.34 & 17.63 & 10.95 & 11.93 & 12.15 & 12.76 \\
\hline $\mathrm{CH}_{3} \bullet$ & + & $\mathrm{CF}_{3} \mathrm{CF}_{3}$ & $\rightarrow$ & $\mathrm{CH}_{2} \mathrm{~F}_{2}$ & + & $\mathrm{CHF}_{2} \mathrm{CF}_{2} \bullet$ & 17.71 & 17.98 & 12.46 & 18.41 & 19.40 & 20.00 & 18.56 \\
\hline $\mathrm{CH}_{3} \cdot$ & & $\mathrm{CF}_{3} \mathrm{CF}_{3}$ & $\rightarrow$ & $\mathrm{CHF}_{2} \mathrm{CF}_{3}$ & + & $\mathrm{CH}_{2} \mathrm{~F} \bullet$ & 12.95 & 11.61 & 8.54 & 11.45 & 12.13 & 12.40 & 12.46 \\
\hline $\mathrm{CH}_{3} \bullet$ & + & $\mathrm{CH}_{3} \mathrm{OH}$ & $\rightarrow$ & $\mathrm{CH}_{2} \cdot \mathrm{OH}$ & + & $\mathrm{CH}_{4}$ & -8.80 & -8.56 & -9.49 & -10.36 & -8.85 & -8.16 & -8.52 \\
\hline $\mathrm{CH}_{3} \bullet$ & + & $\mathrm{CH}_{3} \mathrm{OH}$ & $\rightarrow$ & $\mathrm{CH}_{3} \mathrm{CH}_{2} \mathrm{OH}$ & + & $\mathrm{H} \cdot$ & 8.94 & 8.01 & 13.85 & 7.94 & 8.52 & 9.66 & 9.66 \\
\hline $\mathrm{CH}_{3} \cdot$ & + & $\mathrm{CH}_{2} \mathrm{~F} \bullet$ & $\rightarrow$ & $\mathrm{CH}_{2} \mathrm{FCH}_{3}$ & & & -92.58 & -93.26 & -86.93 & -91.06 & -93.12 & -92.29 & -91.24 \\
\hline $\mathrm{CH}_{3} \bullet$ & + & $\mathrm{CHF}_{2} \cdot$ & $\rightarrow$ & $\mathrm{CHF}_{2} \mathrm{CH}_{3}$ & & & -97.08 & -103.99 & -96.86 & -99.40 & -104.01 & -96.01 & -101.85 \\
\hline $\mathrm{CH}_{3} \cdot$ & + & $\mathrm{CH}_{2} \cdot \mathrm{CH}(=\mathrm{O})$ & $\rightarrow$ & $\mathrm{CH}_{3} \mathrm{CH}_{2} \mathrm{CH}(=\mathrm{O})$ & & & -84.04 & -83.71 & -75.47 & -82.94 & -83.68 & -83.78 & -82.45 \\
\hline $\mathrm{CH}_{3} \cdot$ & + & $\mathrm{CH}_{3} \mathrm{CH}(=\mathrm{O})$ & $\rightarrow$ & $\mathrm{CH}_{3} \mathrm{CH}_{2} \mathrm{CH}(=\mathrm{O})$ & + & $\mathrm{H} \bullet$ & 12.03 & 10.76 & 17.20 & 10.41 & 11.57 & 11.74 & 12.44 \\
\hline
\end{tabular}




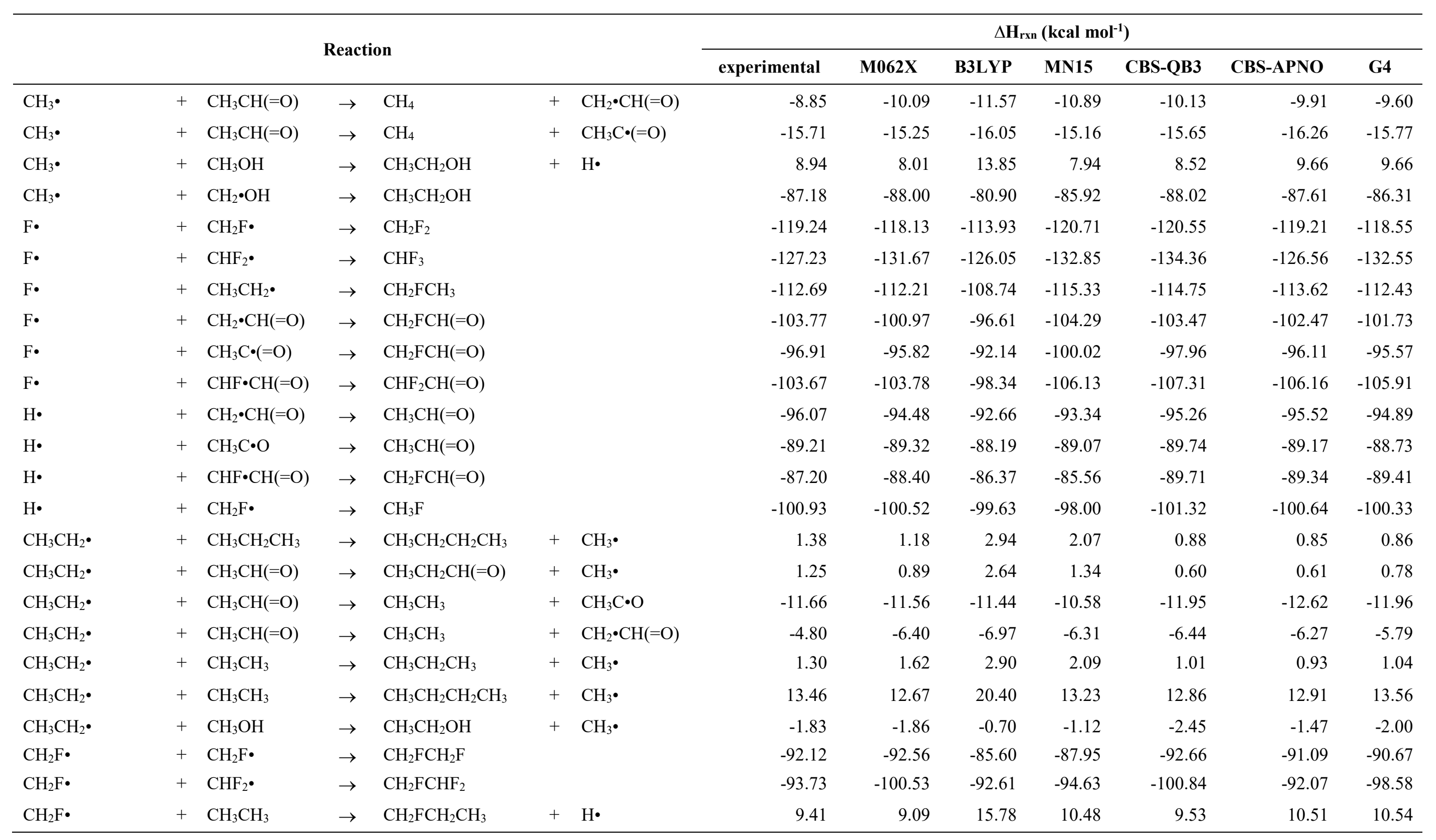




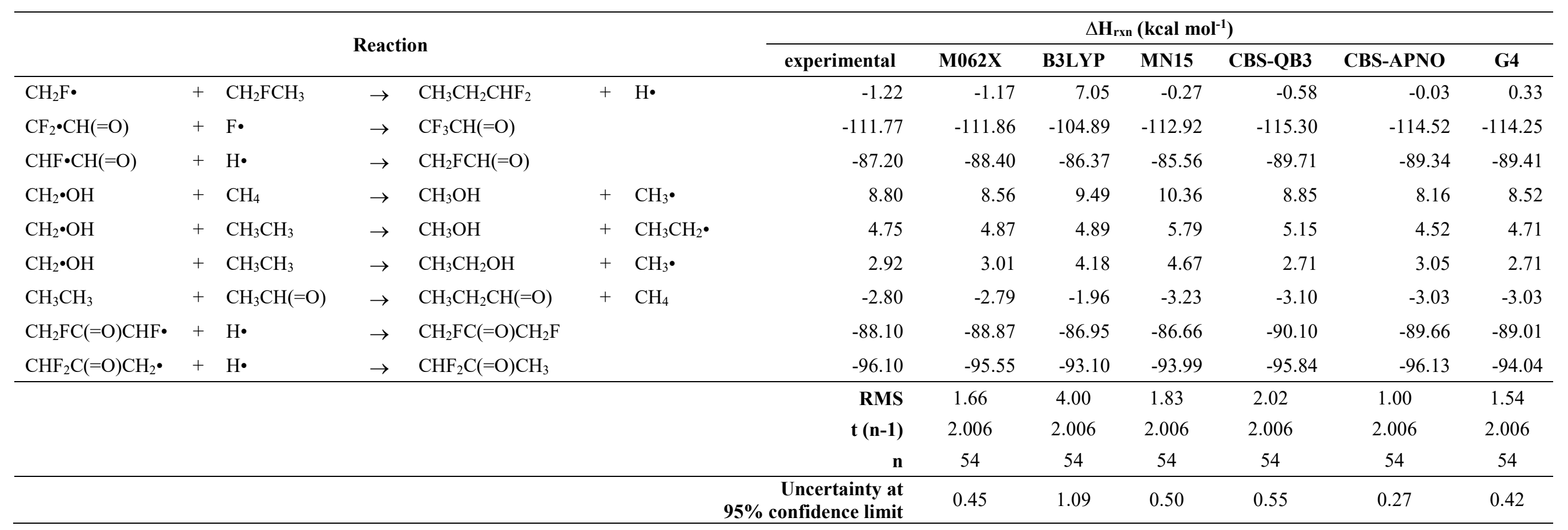




\section{Work reactions for florinated carboxylic acids}

In this work, there are 16 target molecules; 4 species for fluorinated acetic acid and 12 species for fluorinated propionic acid.

The uncertainty is determined from the square root of the sum of squares of the uncertainty in the reference species and the uncertainty of each calculation method

\begin{tabular}{|c|c|c|c|c|c|c|c|c|c|c|}
\hline \multirow{2}{*}{\multicolumn{5}{|c|}{ Isodesmic reactions }} & \multicolumn{6}{|c|}{$\Delta_{f} \mathrm{H}^{\circ}{ }_{298}\left(\mathrm{kcal} \mathrm{mol}^{-1}\right)$} \\
\hline & & & & & \multirow[t]{2}{*}{ M06-2X } & \multirow[t]{2}{*}{ B3LYP } & \multirow[t]{2}{*}{ MN15 } & \multirow[t]{2}{*}{ CBS-QB3 } & \multirow[t]{2}{*}{ CBS-APNO } & \multirow[t]{2}{*}{ G4 } \\
\hline & & $\mathrm{CH}_{3} \mathrm{C}(=\mathrm{O}) \mathrm{OH}$ & & & & & & & & \\
\hline $\mathrm{CH}_{3} \mathrm{C}(=\mathrm{O}) \mathrm{OH}$ & + & $\mathrm{CH}_{3} \mathrm{CH}_{3}$ & $\mathrm{CH}_{3} \mathrm{CH}(=\mathrm{O})$ & $\mathrm{CH}_{3} \mathrm{CH}_{2} \mathrm{OH}$ & -103.81 & -102.91 & -104.46 & -102.93 & -103.51 & -102.71 \\
\hline $\mathrm{CH}_{3} \mathrm{C}(=\mathrm{O}) \mathrm{OH}$ & + & $\mathrm{CH}_{3} \mathrm{CH}_{2} \mathrm{OH}$ & $\mathrm{CH}_{3} \mathrm{CH}_{2} \mathrm{C}(=\mathrm{O}) \mathrm{OH}$ & $\mathrm{CH}_{3} \mathrm{OH}$ & -103.29 & -103.91 & -103.07 & -103.47 & -102.49 & -103.21 \\
\hline $\mathrm{CH}_{3} \mathrm{C}(=\mathrm{O}) \mathrm{OH}$ & + & $\mathrm{CH}_{3} \mathrm{CH}_{2} \mathrm{~F}$ & $\mathrm{CH}_{3} \mathrm{CH}_{2} \mathrm{C}(=\mathrm{O}) \mathrm{OH}$ & $\mathrm{CH}_{3} \mathrm{~F}$ & -103.44 & -104.47 & -103.47 & -103.20 & -103.20 & -103.18 \\
\hline $\mathrm{CH}_{3} \mathrm{C}(=\mathrm{O}) \mathrm{OH}$ & + & $\mathrm{CH}_{3} \mathrm{CH}_{2} \mathrm{CH}_{3}$ & $\mathrm{CH}_{3} \mathrm{CH}_{2} \mathrm{C}(=\mathrm{O}) \mathrm{OH}$ & $\mathrm{CH}_{3} \mathrm{CH}_{3}$ & -102.95 & -103.45 & -102.99 & -103.15 & -103.24 & -103.31 \\
\hline $\mathrm{CH}_{3} \mathrm{C}(=\mathrm{O}) \mathrm{OH}$ & + & $\mathrm{CH}_{3} \mathrm{CH}_{2} \mathrm{CH}(=\mathrm{O})$ & $\mathrm{CH}_{3} \mathrm{CH}_{2} \mathrm{C}(=\mathrm{O}) \mathrm{OH}$ & $\mathrm{CH}_{3} \mathrm{CH}(=\mathrm{O})$ & -103.62 & -103.65 & -103.69 & -103.50 & -103.49 & -103.51 \\
\hline $\mathrm{CH}_{3} \mathrm{C}(=\mathrm{O}) \mathrm{OH}$ & + & $\mathrm{CH}_{3} \mathrm{CH}_{2} \mathrm{CH}_{2} \mathrm{CH}_{2} \mathrm{~F}$ & $\mathrm{CH}_{3} \mathrm{CH}_{2} \mathrm{C}(=\mathrm{O}) \mathrm{OH}$ & $\mathrm{CH}_{3} \mathrm{CH}_{2} \mathrm{CH}_{2} \mathrm{~F}$ & -103.17 & -103.40 & -103.00 & -103.24 & -103.33 & -103.45 \\
\hline $\mathrm{CH}_{3} \mathrm{C}(=\mathrm{O}) \mathrm{OH}$ & + & $\mathrm{CH}_{3} \mathrm{CHF}_{2}$ & $\mathrm{CH}_{3} \mathrm{CH}_{2} \mathrm{C}(=\mathrm{O}) \mathrm{OH}$ & $\mathrm{CH}_{2} \mathrm{~F}_{2}$ & -103.81 & -104.40 & -103.59 & -103.54 & -103.42 & -103.29 \\
\hline $\mathrm{CH}_{3} \mathrm{C}(=\mathrm{O}) \mathrm{OH}$ & + & $\mathrm{CH}_{3} \mathrm{CF}_{3}$ & $\mathrm{CH}_{3} \mathrm{CH}_{2} \mathrm{C}(=\mathrm{O}) \mathrm{OH}$ & $\mathrm{CHF}_{3}$ & -105.58 & -105.61 & -104.98 & -105.33 & -104.89 & -104.84 \\
\hline $\mathrm{CH}_{3} \mathrm{C}(=\mathrm{O}) \mathrm{OH}$ & + & $\mathrm{CHF}_{2} \mathrm{CH}_{2} \mathrm{~F}$ & $\mathrm{CH}_{3} \mathrm{CH}_{2} \mathrm{C}(=\mathrm{O}) \mathrm{OH}$ & $\mathrm{CHF}_{3}$ & -104.46 & -106.80 & -103.22 & -104.36 & -103.08 & -103.65 \\
\hline \multirow[t]{3}{*}{$\mathrm{CH}_{3} \mathrm{C}(=\mathrm{O}) \mathrm{OH}$} & + & $\mathrm{CH}_{3} \mathrm{CH}_{2} \mathrm{CH}_{2} \mathrm{CH}_{3}$ & $\mathrm{CH}_{3} \mathrm{CH}_{2} \mathrm{C}(=\mathrm{O}) \mathrm{OH}$ & $\mathrm{CH}_{3} \mathrm{CH}_{2} \mathrm{CH}_{3}$ & -103.45 & -103.47 & -103.08 & -103.34 & -103.38 & -103.55 \\
\hline & & & & Average & -103.76 & -104.21 & -103.55 & -103.61 & -103.40 & -103.47 \\
\hline & & & & Uncertainty & 0.63 & 1.20 & 0.67 & 0.71 & 0.49 & 0.60 \\
\hline & & $\mathrm{CH}_{2} \mathrm{FC}(=\mathrm{O}) \mathrm{OH}$ & & & & & & & & \\
\hline $\mathrm{CH}_{2} \mathrm{FC}(=\mathrm{O}) \mathrm{OH}$ & + & $\mathrm{CH}_{3} \mathrm{CH}_{3}$ & $\mathrm{CH}_{3} \mathrm{C}(=\mathrm{O}) \mathrm{OH}$ & $\mathrm{CH}_{3} \mathrm{CH}_{2} \mathrm{~F}$ & -141.72 & -141.22 & -142.28 & -141.77 & -142.34 & -141.79 \\
\hline $\mathrm{CH}_{2} \mathrm{FC}(=\mathrm{O}) \mathrm{OH}$ & + & $\mathrm{CH}_{3} \mathrm{CH}(=\mathrm{O})$ & $\rightarrow \quad \mathrm{CH}_{3} \mathrm{C}(=\mathrm{O}) \mathrm{OH}$ & $\mathrm{CH}_{2} \mathrm{FCH}(=\mathrm{O})$ & -142.44 & -142.25 & -142.88 & -142.49 & -142.51 & -142.58 \\
\hline $\mathrm{CH}_{2} \mathrm{FC}(=\mathrm{O}) \mathrm{OH}$ & + & $\mathrm{CH}_{3} \mathrm{OH}$ & $\rightarrow \quad \mathrm{CH}_{3} \mathrm{C}(=\mathrm{O}) \mathrm{OH}$ & $\mathrm{CH}_{2} \mathrm{FOH}$ & -141.97 & -142.07 & -142.30 & -142.08 & -142.74 & -142.24 \\
\hline $\mathrm{CH}_{2} \mathrm{FC}(=\mathrm{O}) \mathrm{OH}$ & + & $\mathrm{CH}_{4}$ & $\rightarrow \quad \mathrm{CH}_{3} \mathrm{C}(=\mathrm{O}) \mathrm{OH}$ & $\mathrm{CH}_{3} \mathrm{~F}$ & -141.54 & -141.20 & -142.49 & -141.76 & -141.75 & -141.70 \\
\hline $\mathrm{CH}_{2} \mathrm{FC}(=\mathrm{O}) \mathrm{OH}$ & + & $\mathrm{CH}_{3} \mathrm{CH}_{2} \mathrm{CH}_{3}$ & $\mathrm{CH}_{3} \mathrm{C}(=\mathrm{O}) \mathrm{OH}$ & $\mathrm{CH}_{3} \mathrm{CH}_{2} \mathrm{CH}_{2} \mathrm{~F}$ & -141.76 & -141.58 & -142.26 & -141.90 & -142.08 & -142.04 \\
\hline $\mathrm{CH}_{2} \mathrm{FC}(=\mathrm{O}) \mathrm{OH}$ & + & $\mathrm{CH}_{3} \mathrm{CH}_{2} \mathrm{CH}_{2} \mathrm{CH}_{3}$ & $\rightarrow \quad \mathrm{CH}_{3} \mathrm{C}(=\mathrm{O}) \mathrm{OH}$ & $\mathrm{CH}_{3} \mathrm{CH}_{2} \mathrm{CH}_{2} \mathrm{CH}_{2} \mathrm{~F}$ & -142.04 & -141.65 & -142.34 & -142.00 & -142.13 & -142.14 \\
\hline $\mathrm{CH}_{2} \mathrm{FC}(=\mathrm{O}) \mathrm{OH}$ & + & $\mathrm{CH}_{3} \mathrm{CH}(=\mathrm{O})$ & $\rightarrow \quad \mathrm{CH}_{3} \mathrm{C}(=\mathrm{O}) \mathrm{OH}$ & $\mathrm{CH}_{3} \mathrm{CF}(=\mathrm{O})$ & -142.95 & -143.56 & -142.16 & -142.80 & -142.06 & -142.72 \\
\hline $\mathrm{CH}_{2} \mathrm{FC}(=\mathrm{O}) \mathrm{OH}$ & + & $\mathrm{CH}_{3} \mathrm{CH}_{2} \mathrm{CH}(=\mathrm{O})$ & $\mathrm{CH}_{3} \mathrm{C}(=\mathrm{O}) \mathrm{OH}$ & $\mathrm{CH}_{2} \mathrm{FCH}_{2} \mathrm{CH}(=\mathrm{O})$ & -141.98 & -141.64 & -141.72 & -141.76 & -141.48 & -141.83 \\
\hline \multirow[t]{3}{*}{$\mathrm{CH}_{2} \mathrm{FC}(=\mathrm{O}) \mathrm{OH}$} & + & $\mathrm{CH}_{3} \mathrm{C}(=\mathrm{O}) \mathrm{CH}_{3}$ & $\mathrm{CH}_{3} \mathrm{C}(=\mathrm{O}) \mathrm{OH}$ & $\mathrm{CH}_{2} \mathrm{FC}(=\mathrm{O}) \mathrm{CH}_{3}$ & -143.14 & -143.04 & -142.74 & -143.38 & -142.97 & -142.97 \\
\hline & & & & Average & -142.17 & -142.03 & -142.36 & -142.22 & -142.23 & -142.22 \\
\hline & & & & Uncertainty & 0.87 & 1.35 & 0.90 & 0.93 & 0.77 & 0.85 \\
\hline
\end{tabular}




\begin{tabular}{|c|c|c|c|c|c|c|c|c|c|c|c|c|}
\hline \multirow{2}{*}{\multicolumn{7}{|c|}{ Isodesmic reactions }} & \multicolumn{6}{|c|}{$\Delta_{f} \mathbf{H}^{\circ}{ }_{298}\left(\mathrm{kcal} \mathrm{mol}^{-1}\right)$} \\
\hline & & & & & & & \multirow[t]{2}{*}{ M06-2X } & \multirow[t]{2}{*}{ B3LYP } & \multirow[t]{2}{*}{ MN15 } & \multirow[t]{2}{*}{ CBS-QB3 } & \multirow[t]{2}{*}{ CBS-APNO } & \multirow[t]{2}{*}{ G4 } \\
\hline & & $\mathrm{CHF}_{2} \mathrm{C}(=\mathrm{O}) \mathrm{OH}$ & & & & & & & & & & \\
\hline $\mathrm{CHF}_{2} \mathrm{C}(=\mathrm{O}) \mathrm{OH}$ & + & $\mathrm{CH}_{3} \mathrm{CH}_{3}$ & $\rightarrow$ & $\mathrm{CH}_{3} \mathrm{C}(=\mathrm{O}) \mathrm{OH}$ & + & $\mathrm{CH}_{3} \mathrm{CHF}_{2}$ & -189.53 & -189.48 & -190.30 & -190.09 & -190.08 & -190.21 \\
\hline $\mathrm{CHF}_{2} \mathrm{C}(=\mathrm{O}) \mathrm{OH}$ & + & $\mathrm{CH}_{3} \mathrm{CH}(=\mathrm{O})$ & $\rightarrow$ & $\mathrm{CH}_{3} \mathrm{C}(=\mathrm{O}) \mathrm{OH}$ & + & $\mathrm{CHF}_{2} \mathrm{CH}(=\mathrm{O})$ & -190.99 & -190.93 & -191.30 & -190.94 & -190.97 & -190.98 \\
\hline $\mathrm{CHF}_{2} \mathrm{C}(=\mathrm{O}) \mathrm{OH}$ & + & $\mathrm{CH}_{4}$ & $\rightarrow$ & $\mathrm{CH}_{3} \mathrm{C}(=\mathrm{O}) \mathrm{OH}$ & + & $\mathrm{CH}_{2} \mathrm{~F}_{2}$ & -189.71 & -189.39 & -190.62 & -190.43 & -190.24 & -190.22 \\
\hline $\mathrm{CHF}_{2} \mathrm{C}(=\mathrm{O}) \mathrm{OH}$ & + & $\mathrm{CH}_{3} \mathrm{CH}_{2} \mathrm{CH}_{3}$ & $\rightarrow$ & $\mathrm{CH}_{3} \mathrm{C}(=\mathrm{O}) \mathrm{OH}$ & + & $\mathrm{CH}_{3} \mathrm{CH}_{2} \mathrm{CHF}_{2}$ & -190.08 & -190.37 & -190.81 & -190.76 & -190.89 & -190.96 \\
\hline $\mathrm{CHF}_{2} \mathrm{C}(=\mathrm{O}) \mathrm{OH}$ & + & $\mathrm{CH}_{3} \mathrm{CH}_{2} \mathrm{CH}_{3}$ & $\rightarrow$ & $\mathrm{CH}_{3} \mathrm{C}(=\mathrm{O}) \mathrm{OH}$ & + & $\mathrm{CH}_{3} \mathrm{CF}_{2} \mathrm{CH}_{3}$ & -189.97 & -190.76 & -191.17 & -190.65 & -190.90 & -191.01 \\
\hline $\mathrm{CHF}_{2} \mathrm{C}(=\mathrm{O}) \mathrm{OH}$ & + & $\mathrm{CH}_{3} \mathrm{CH}(=\mathrm{O})$ & $\rightarrow$ & $\mathrm{CH}_{3} \mathrm{C}(=\mathrm{O}) \mathrm{OH}$ & + & $\mathrm{CH}_{2} \mathrm{FCF}(=\mathrm{O})$ & -191.59 & -191.46 & -190.71 & -191.45 & -191.08 & -191.48 \\
\hline $\mathrm{CHF}_{2} \mathrm{C}(=\mathrm{O}) \mathrm{OH}$ & + & $\mathrm{CH}_{3} \mathrm{CH}_{2} \mathrm{CH}(=\mathrm{O})$ & $\rightarrow$ & $\mathrm{CH}_{3} \mathrm{C}(=\mathrm{O}) \mathrm{OH}$ & + & $\mathrm{CHF}_{2} \mathrm{CH}_{2} \mathrm{CH}(=\mathrm{O})$ & -189.73 & -189.90 & -190.20 & -190.06 & -190.17 & -190.20 \\
\hline \multirow[t]{4}{*}{$\mathrm{CHF}_{2} \mathrm{C}(=\mathrm{O}) \mathrm{OH}$} & + & $\mathrm{CH}_{3} \mathrm{C}(=\mathrm{O}) \mathrm{CH}_{3}$ & $\rightarrow$ & $\mathrm{CH}_{3} \mathrm{C}(=\mathrm{O}) \mathrm{OH}$ & + & $\mathrm{CHF}_{2} \mathrm{C}(=\mathrm{O}) \mathrm{CH}_{3}$ & -190.98 & -191.73 & -191.21 & -191.75 & -191.39 & -191.36 \\
\hline & & & & & & Average & -190.32 & -190.50 & -190.79 & -190.77 & -190.71 & -190.80 \\
\hline & & & & & & Uncertainty & 0.64 & 1.19 & 0.68 & 0.72 & 0.53 & 0.62 \\
\hline & & $\mathrm{CF}_{3} \mathrm{C}(=\mathrm{O}) \mathrm{OH}$ & & & & & & & & & & \\
\hline $\mathrm{CF}_{3} \mathrm{C}(=\mathrm{O}) \mathrm{OH}$ & + & $\mathrm{CH}_{3} \mathrm{CH}_{3}$ & $\rightarrow$ & $\mathrm{CH}_{3} \mathrm{C}(=\mathrm{O}) \mathrm{OH}$ & + & $\mathrm{CH}_{3} \mathrm{CF}_{3}$ & -243.34 & -243.28 & -244.61 & -243.99 & -244.12 & -244.31 \\
\hline $\mathrm{CF}_{3} \mathrm{C}(=\mathrm{O}) \mathrm{OH}$ & + & $\mathrm{CH}_{4}$ & $\rightarrow$ & $\mathrm{CH}_{3} \mathrm{C}(=\mathrm{O}) \mathrm{OH}$ & + & $\mathrm{CHF}_{3}$ & -245.30 & -244.40 & -246.33 & -246.11 & -245.75 & -245.87 \\
\hline $\mathrm{CF}_{3} \mathrm{C}(=\mathrm{O}) \mathrm{OH}$ & + & $\mathrm{CH}_{3} \mathrm{CH}_{2} \mathrm{CH}_{3}$ & $\rightarrow$ & $\mathrm{CH}_{3} \mathrm{C}(=\mathrm{O}) \mathrm{OH}$ & + & $\mathrm{CH}_{3} \mathrm{CH}_{2} \mathrm{CF}_{3}$ & -245.44 & -245.77 & -246.52 & -246.01 & -246.43 & -246.47 \\
\hline $\mathrm{CF}_{3} \mathrm{C}(=\mathrm{O}) \mathrm{OH}$ & + & $\mathrm{CH}_{3} \mathrm{CH}(=\mathrm{O})$ & $\rightarrow$ & $\mathrm{CH}_{3} \mathrm{C}(=\mathrm{O}) \mathrm{OH}$ & + & $\mathrm{CF}_{3} \mathrm{CH}(=\mathrm{O})$ & -246.35 & -246.23 & -246.71 & -246.19 & -246.21 & -246.23 \\
\hline $\mathrm{CF}_{3} \mathrm{C}(=\mathrm{O}) \mathrm{OH}$ & + & $\mathrm{CH}_{3} \mathrm{CH}(=\mathrm{O})$ & $\rightarrow$ & $\mathrm{CH}_{3} \mathrm{C}(=\mathrm{O}) \mathrm{OH}$ & + & $\mathrm{CHF}_{2} \mathrm{CF}(=\mathrm{O})$ & -246.90 & -245.38 & -246.77 & -246.44 & -246.56 & -246.74 \\
\hline $\mathrm{CF}_{3} \mathrm{C}(=\mathrm{O}) \mathrm{OH}$ & + & $\mathrm{CH}_{3} \mathrm{CH}_{2} \mathrm{CH}(=\mathrm{O})$ & $\rightarrow$ & $\mathrm{CH}_{3} \mathrm{C}(=\mathrm{O}) \mathrm{OH}$ & + & $\mathrm{CF}_{3} \mathrm{CH}_{2} \mathrm{CH}(=\mathrm{O})$ & -245.17 & -245.13 & -246.22 & -245.33 & -245.62 & -245.70 \\
\hline $\mathrm{CF}_{3} \mathrm{C}(=\mathrm{O}) \mathrm{OH}$ & + & $\mathrm{CH}_{3} \mathrm{CH}_{2} \mathrm{CH}(=\mathrm{O})$ & $\rightarrow$ & $\mathrm{CH}_{3} \mathrm{C}(=\mathrm{O}) \mathrm{OH}$ & + & $\mathrm{CHF}_{2} \mathrm{CHFCH}(=\mathrm{O})$ & -245.69 & -243.90 & -247.23 & -245.63 & -246.76 & -246.28 \\
\hline \multirow[t]{3}{*}{$\mathrm{CF}_{3} \mathrm{C}(=\mathrm{O}) \mathrm{OH}$} & + & $\mathrm{CH}_{3} \mathrm{C}(=\mathrm{O}) \mathrm{CH}_{3}$ & $\rightarrow$ & $\mathrm{CH}_{3} \mathrm{C}(=\mathrm{O}) \mathrm{OH}$ & + & $\mathrm{CF}_{3} \mathrm{C}(=\mathrm{O}) \mathrm{CH}_{3}$ & -246.76 & -246.90 & -246.52 & -246.83 & -246.59 & -246.49 \\
\hline & & & & & & Average & -245.62 & -245.13 & -246.36 & -245.82 & -246.01 & -246.01 \\
\hline & & & & & & Uncertainty & 0.73 & 1.24 & 0.76 & 0.80 & 0.62 & 0.71 \\
\hline
\end{tabular}




\begin{tabular}{|c|c|c|c|c|c|c|c|c|c|c|c|}
\hline \multirow{2}{*}{\multicolumn{6}{|c|}{ Isodesmic reactions }} & \multicolumn{6}{|c|}{$\Delta_{f} \mathbf{H}^{\circ}{ }_{298}\left(\mathrm{kcal} \mathrm{mol}^{-1}\right)$} \\
\hline & & & & & & \multirow[t]{2}{*}{ M06-2X } & \multirow[t]{2}{*}{ B3LYP } & \multirow[t]{2}{*}{ MN15 } & \multirow[t]{2}{*}{ CBS-QB3 } & \multirow[t]{2}{*}{ CBS-APNO } & \multirow[t]{2}{*}{ G4 } \\
\hline & & $\mathrm{CH}_{3} \mathrm{CH}_{2} \mathrm{C}(=\mathrm{O}) \mathrm{OH}$ & & & & & & & & & \\
\hline $\mathrm{CH}_{3} \mathrm{CH}_{2} \mathrm{C}(=\mathrm{O}) \mathrm{OH}$ & + & $\mathrm{CH}_{3} \mathrm{OH}$ & $\rightarrow$ & $\mathrm{CH}_{3} \mathrm{C}(=\mathrm{O}) \mathrm{OH}$ & $\mathrm{CH}_{3} \mathrm{CH}_{2} \mathrm{OH}$ & -108.72 & -108.09 & -108.93 & -108.54 & -109.52 & -108.80 \\
\hline $\mathrm{CH}_{3} \mathrm{CH}_{2} \mathrm{C}(=\mathrm{O}) \mathrm{OH}$ & + & $\mathrm{CH}_{3} \mathrm{CH}(=\mathrm{O})$ & $\rightarrow$ & $\mathrm{CH}_{3} \mathrm{C}(=\mathrm{O}) \mathrm{OH}$ & $+\mathrm{CH}_{3} \mathrm{CH}_{2} \mathrm{CH}(=\mathrm{O})$ & -108.39 & -108.35 & -108.31 & -108.50 & -108.51 & -108.50 \\
\hline $\mathrm{CH}_{3} \mathrm{CH}_{2} \mathrm{C}(=\mathrm{O}) \mathrm{OH}$ & + & $\mathrm{CH}_{3} \mathrm{CH}_{2} \mathrm{~F}$ & $\rightarrow$ & $\mathrm{CH}_{3} \mathrm{C}(=\mathrm{O}) \mathrm{OH}$ & $+\mathrm{CH}_{3} \mathrm{CH}_{2} \mathrm{CH}_{2} \mathrm{~F}$ & -109.08 & -108.90 & -108.97 & -108.97 & -109.03 & -108.93 \\
\hline $\mathrm{CH}_{3} \mathrm{CH}_{2} \mathrm{C}(=\mathrm{O}) \mathrm{OH}$ & + & $\mathrm{CH}_{4}$ & $\rightarrow$ & $\mathrm{CH}_{3} \mathrm{C}(=\mathrm{O}) \mathrm{OH}$ & $+\mathrm{CH}_{3} \mathrm{CH}_{3}$ & -108.37 & -107.50 & -108.75 & -108.79 & -108.73 & -108.72 \\
\hline $\mathrm{CH}_{3} \mathrm{CH}_{2} \mathrm{C}(=\mathrm{O}) \mathrm{OH}$ & + & $\mathrm{CH}_{3} \mathrm{CH}_{3}$ & $\rightarrow$ & $\mathrm{CH}_{3} \mathrm{C}(=\mathrm{O}) \mathrm{OH}$ & $+\mathrm{CH}_{3} \mathrm{CH}_{2} \mathrm{CH}_{3}$ & -109.05 & -108.54 & -109.02 & -108.84 & -108.76 & -108.69 \\
\hline $\mathrm{CH}_{3} \mathrm{CH}_{2} \mathrm{C}(=\mathrm{O}) \mathrm{OH}$ & + & $\mathrm{CH}_{3} \mathrm{~F}$ & $\rightarrow$ & $\mathrm{CH}_{3} \mathrm{C}(=\mathrm{O}) \mathrm{OH}$ & $+\mathrm{CH}_{3} \mathrm{CH}_{2} \mathrm{~F}$ & -108.55 & -107.53 & -108.52 & -108.80 & -108.79 & -108.81 \\
\hline $\mathrm{CH}_{3} \mathrm{CH}_{2} \mathrm{C}(=\mathrm{O}) \mathrm{OH}$ & + & $\mathrm{CH}_{2} \mathrm{~F}_{2}$ & $\rightarrow$ & $\mathrm{CH}_{3} \mathrm{C}(=\mathrm{O}) \mathrm{OH}$ & $+\mathrm{CH}_{3} \mathrm{CHF}_{2}$ & -108.18 & -107.60 & -108.41 & -108.45 & -108.57 & -108.70 \\
\hline $\mathrm{CH}_{3} \mathrm{CH}_{2} \mathrm{C}(=\mathrm{O}) \mathrm{OH}$ & + & $\mathrm{CHF}_{3}$ & $\rightarrow$ & $\mathrm{CH}_{3} \mathrm{C}(=\mathrm{O}) \mathrm{OH}$ & $+\mathrm{CH}_{3} \mathrm{CF}_{3}$ & -106.42 & -106.38 & -107.01 & -106.67 & -107.10 & -107.15 \\
\hline $\mathrm{CH}_{3} \mathrm{CH}_{2} \mathrm{C}(=\mathrm{O}) \mathrm{OH}$ & + & $\mathrm{CH}_{3} \mathrm{CHF}_{2}$ & $\rightarrow$ & $\mathrm{CH}_{3} \mathrm{C}(=\mathrm{O}) \mathrm{OH}$ & $+\mathrm{CH}_{3} \mathrm{CH}_{2} \mathrm{CHF}_{2}$ & -109.60 & -109.43 & -109.51 & -109.51 & -109.57 & -109.44 \\
\hline \multirow[t]{4}{*}{$\mathrm{CH}_{3} \mathrm{CH}_{2} \mathrm{C}(=\mathrm{O}) \mathrm{OH}$} & + & $\mathrm{CH}_{3} \mathrm{CH}_{2} \mathrm{CH}_{3}$ & $\rightarrow$ & $\mathrm{CH}_{3} \mathrm{C}(=\mathrm{O}) \mathrm{OH}$ & $+\mathrm{CH}_{3} \mathrm{CH}_{2} \mathrm{CH}_{2} \mathrm{CH}_{3}$ & -108.54 & -108.52 & -108.91 & -108.65 & -108.62 & -108.45 \\
\hline & & & & & Average & -108.49 & -108.08 & -108.63 & -108.57 & -108.72 & -108.62 \\
\hline & & & & & Uncertainty & 0.60 & 1.18 & 0.64 & 0.68 & 0.45 & 0.57 \\
\hline & & $\mathrm{CH}_{3} \mathrm{CHFC}(=\mathrm{O}) \mathrm{OH}$ & & & & & & & & & \\
\hline $\mathrm{CH}_{3} \mathrm{CHFC}(=\mathrm{O}) \mathrm{OH}$ & + & $\mathrm{CH}_{3} \mathrm{CH}_{3}$ & $\rightarrow$ & $\mathrm{CH}_{3} \mathrm{CH}_{2} \mathrm{C}(=\mathrm{O}) \mathrm{OH}$ & $+\mathrm{CH}_{3} \mathrm{CH}_{2} \mathrm{~F}$ & -151.23 & -150.79 & -151.78 & -151.32 & -151.36 & -151.32 \\
\hline $\mathrm{CH}_{3} \mathrm{CHFC}(=\mathrm{O}) \mathrm{OH}$ & + & $\mathrm{CH}_{3} \mathrm{CHF}_{2}$ & $\rightarrow$ & $\mathrm{CH}_{3} \mathrm{CH}_{2} \mathrm{C}(=\mathrm{O}) \mathrm{OH}$ & $+\mathrm{CH}_{2} \mathrm{FCHF}_{2}$ & -151.10 & -151.05 & -151.19 & -151.05 & -151.10 & -150.97 \\
\hline $\mathrm{CH}_{3} \mathrm{CHFC}(=\mathrm{O}) \mathrm{OH}$ & + & $\mathrm{CH}_{3} \mathrm{CH}_{2} \mathrm{~F}$ & $\rightarrow$ & $\mathrm{CH}_{3} \mathrm{CH}_{2} \mathrm{C}(=\mathrm{O}) \mathrm{OH}$ & $+\mathrm{CH}_{2} \mathrm{FCH}_{2} \mathrm{~F}$ & -151.23 & -151.01 & -152.41 & -151.22 & -151.24 & -151.16 \\
\hline $\mathrm{CH}_{3} \mathrm{CHFC}(=\mathrm{O}) \mathrm{OH}$ & + & $\mathrm{CH}_{4}$ & $\rightarrow$ & $\mathrm{CH}_{3} \mathrm{CH}_{2} \mathrm{C}(=\mathrm{O}) \mathrm{OH}$ & $+\mathrm{CH}_{3} \mathrm{~F}$ & -151.05 & -150.77 & -152.00 & -151.31 & -151.30 & -151.23 \\
\hline $\mathrm{CH}_{3} \mathrm{CHFC}(=\mathrm{O}) \mathrm{OH}$ & + & $\mathrm{CH}_{3} \mathrm{CH}_{2} \mathrm{CH}_{3}$ & $\rightarrow$ & $\mathrm{CH}_{3} \mathrm{CH}_{2} \mathrm{C}(=\mathrm{O}) \mathrm{OH}$ & $+\mathrm{CH}_{3} \mathrm{CH}_{2} \mathrm{CH}_{2} \mathrm{~F}$ & -151.26 & -151.15 & -151.76 & -151.45 & -151.63 & -151.57 \\
\hline $\mathrm{CH}_{3} \mathrm{CHFC}(=\mathrm{O}) \mathrm{OH}$ & + & $\mathrm{CH}_{3} \mathrm{CH}_{2} \mathrm{CH}_{2} \mathrm{CH}_{3}$ & $\rightarrow$ & $\mathrm{CH}_{3} \mathrm{CH}_{2} \mathrm{C}(=\mathrm{O}) \mathrm{OH}$ & $+\mathrm{CH}_{3} \mathrm{CH}_{2} \mathrm{CH}_{2} \mathrm{CH}_{2} \mathrm{~F}$ & -151.55 & -151.22 & -151.84 & -151.55 & -151.68 & -151.67 \\
\hline $\mathrm{CH}_{3} \mathrm{CHFC}(=\mathrm{O}) \mathrm{OH}$ & + & $\mathrm{CH}_{3} \mathrm{CH}(=\mathrm{O})$ & $\rightarrow$ & $\mathrm{CH}_{3} \mathrm{CH}_{2} \mathrm{C}(=\mathrm{O}) \mathrm{OH}$ & $+\mathrm{CH}_{2} \mathrm{FCH}(=\mathrm{O})$ & -151.94 & -151.82 & -152.39 & -152.04 & -152.13 & -152.11 \\
\hline $\mathrm{CH}_{3} \mathrm{CHFC}(=\mathrm{O}) \mathrm{OH}$ & + & $\mathrm{CH}_{3} \mathrm{CH}(=\mathrm{O})$ & $\rightarrow$ & $\mathrm{CH}_{3} \mathrm{CH}_{2} \mathrm{C}(=\mathrm{O}) \mathrm{OH}$ & $+\mathrm{CH}_{3} \mathrm{CF}(=\mathrm{O})$ & -152.46 & -153.13 & -151.66 & -152.35 & -151.61 & -152.25 \\
\hline $\mathrm{CH}_{3} \mathrm{CHFC}(=\mathrm{O}) \mathrm{OH}$ & + & $\mathrm{CH}_{3} \mathrm{CH}_{2} \mathrm{CH}(=\mathrm{O})$ & $\rightarrow$ & $\mathrm{CH}_{3} \mathrm{CH}_{2} \mathrm{C}(=\mathrm{O}) \mathrm{OH}$ & $+\mathrm{CH}_{2} \mathrm{FCH}_{2} \mathrm{CH}(=\mathrm{O})$ & -151.48 & -151.21 & -151.23 & -151.31 & -151.03 & -151.36 \\
\hline \multirow[t]{3}{*}{$\mathrm{CH}_{3} \mathrm{CHFC}(=\mathrm{O}) \mathrm{OH}$} & + & $\mathrm{CH}_{3} \mathrm{C}(=\mathrm{O}) \mathrm{CH}_{3}$ & $\rightarrow$ & $\mathrm{CH}_{3} \mathrm{CH}_{2} \mathrm{C}(=\mathrm{O}) \mathrm{OH}$ & $+\mathrm{CH}_{2} \mathrm{FC}(=\mathrm{O}) \mathrm{CH}_{3}$ & -152.64 & -152.61 & -152.25 & -152.93 & -152.52 & -152.50 \\
\hline & & & & & Average & -151.59 & -151.48 & -151.85 & -151.65 & -151.56 & -151.61 \\
\hline & & & & & Uncertainty & 0.88 & 1.32 & 0.90 & 0.94 & 0.78 & 0.86 \\
\hline
\end{tabular}




\begin{tabular}{|c|c|c|c|c|c|c|c|c|c|c|c|c|}
\hline \multirow{2}{*}{\multicolumn{7}{|c|}{ Isodesmic reactions }} & \multicolumn{6}{|c|}{$\Delta_{f} \mathbf{H}^{\circ}{ }_{298}\left(\mathrm{kcal} \mathrm{mol}^{-1}\right)$} \\
\hline & & & & & & & \multirow[t]{2}{*}{ M06-2X } & \multirow[t]{2}{*}{ B3LYP } & \multirow[t]{2}{*}{ MN15 } & \multirow[t]{2}{*}{ CBS-QB3 } & \multirow[t]{2}{*}{ CBS-APNO } & \multirow[t]{2}{*}{ G4 } \\
\hline & & $\mathrm{CH}_{3} \mathrm{CF}_{2} \mathrm{C}(=\mathrm{O}) \mathrm{OH}$ & & & & & & & & & & \\
\hline $\mathrm{CH}_{3} \mathrm{CF}_{2} \mathrm{C}(=\mathrm{O}) \mathrm{OH}$ & + & $\mathrm{CH}_{3} \mathrm{CHF}_{2}$ & $\rightarrow$ & $\mathrm{CH}_{3} \mathrm{CH}_{2} \mathrm{C}(=\mathrm{O}) \mathrm{OH}$ & + & $\mathrm{CHF}_{2} \mathrm{CHF}_{2}$ & -203.17 & -203.07 & -202.71 & -203.35 & -203.06 & -203.06 \\
\hline $\mathrm{CH}_{3} \mathrm{CF}_{2} \mathrm{C}(=\mathrm{O}) \mathrm{OH}$ & + & $\mathrm{CH}_{3} \mathrm{CH}_{3}$ & $\rightarrow$ & $\mathrm{CH}_{3} \mathrm{CH}_{2} \mathrm{C}(=\mathrm{O}) \mathrm{OH}$ & + & $\mathrm{CH}_{3} \mathrm{CHF}_{2}$ & -203.35 & -202.62 & -203.77 & -203.85 & -203.55 & -203.72 \\
\hline $\mathrm{CH}_{3} \mathrm{CF}_{2} \mathrm{C}(=\mathrm{O}) \mathrm{OH}$ & + & $\mathrm{CH}_{3} \mathrm{CH}_{2} \mathrm{~F}$ & $\rightarrow$ & $\mathrm{CH}_{3} \mathrm{CH}_{2} \mathrm{C}(=\mathrm{O}) \mathrm{OH}$ & + & $\mathrm{CH}_{3} \mathrm{CF}_{3}$ & -202.11 & -204.08 & -201.41 & -202.61 & -201.48 & -202.17 \\
\hline $\mathrm{CH}_{3} \mathrm{CF}_{2} \mathrm{C}(=\mathrm{O}) \mathrm{OH}$ & + & $\mathrm{CH}_{4}$ & $\rightarrow$ & $\mathrm{CH}_{3} \mathrm{CH}_{2} \mathrm{C}(=\mathrm{O}) \mathrm{OH}$ & + & $\mathrm{CH}_{2} \mathrm{~F}_{2}$ & -203.54 & -202.53 & -204.10 & -204.19 & -203.71 & -203.73 \\
\hline $\mathrm{CH}_{3} \mathrm{CF}_{2} \mathrm{C}(=\mathrm{O}) \mathrm{OH}$ & + & $\mathrm{CH}_{3} \mathrm{CH}_{2} \mathrm{CH}_{3}$ & $\rightarrow$ & $\mathrm{CH}_{3} \mathrm{CH}_{2} \mathrm{C}(=\mathrm{O}) \mathrm{OH}$ & + & $\mathrm{CH}_{3} \mathrm{CF}_{2} \mathrm{CH}_{3}$ & -203.79 & -203.91 & -204.65 & -204.41 & -204.38 & -204.52 \\
\hline $\mathrm{CH}_{3} \mathrm{CF}_{2} \mathrm{C}(=\mathrm{O}) \mathrm{OH}$ & + & $\mathrm{CH}_{3} \mathrm{CH}(=\mathrm{O})$ & $\rightarrow$ & $\mathrm{CH}_{3} \mathrm{CH}_{2} \mathrm{C}(=\mathrm{O}) \mathrm{OH}$ & + & $\mathrm{CHF}_{2} \mathrm{CH}(=\mathrm{O})$ & -204.82 & -204.07 & -204.77 & -204.70 & -204.44 & -204.49 \\
\hline $\mathrm{CH}_{3} \mathrm{CF}_{2} \mathrm{C}(=\mathrm{O}) \mathrm{OH}$ & + & $\mathrm{CH}_{3} \mathrm{CH}(=\mathrm{O})$ & $\rightarrow$ & $\mathrm{CH}_{3} \mathrm{CH}_{2} \mathrm{C}(=\mathrm{O}) \mathrm{OH}$ & + & $\mathrm{CH}_{2} \mathrm{FCF}(=\mathrm{O})$ & -205.41 & -204.60 & -204.19 & -205.20 & -204.56 & -204.99 \\
\hline $\mathrm{CH}_{3} \mathrm{CF}_{2} \mathrm{C}(=\mathrm{O}) \mathrm{OH}$ & + & $\mathrm{CH}_{3} \mathrm{CH}_{2} \mathrm{CH}(=\mathrm{O})$ & $\rightarrow$ & $\mathrm{CH}_{3} \mathrm{CH}_{2} \mathrm{C}(=\mathrm{O}) \mathrm{OH}$ & + & $\mathrm{CHF}_{2} \mathrm{CH}_{2} \mathrm{CH}(=\mathrm{O})$ & -203.56 & -203.04 & -203.67 & -203.82 & -203.64 & -203.71 \\
\hline \multirow[t]{3}{*}{$\mathrm{CH}_{3} \mathrm{CF}_{2} \mathrm{C}(=\mathrm{O}) \mathrm{OH}$} & + & $\mathrm{CH}_{3} \mathrm{C}(=\mathrm{O}) \mathrm{CH}_{3}$ & $\rightarrow$ & $\mathrm{CH}_{3} \mathrm{CH}_{2} \mathrm{C}(=\mathrm{O}) \mathrm{OH}$ & + & $\mathrm{CHF}_{2} \mathrm{C}(=\mathrm{O}) \mathrm{CH}_{3}$ & -204.80 & -204.87 & -204.69 & -205.50 & -204.87 & -204.87 \\
\hline & & & & & & Average & -203.85 & -203.63 & -203.83 & -204.21 & -203.81 & -203.97 \\
\hline & & $\mathrm{CH}_{2} \mathrm{FCH}_{2} \mathrm{C}(=\mathrm{O}) \mathrm{OI}$ & & & & & & & & & & \\
\hline $\mathrm{CH}_{2} \mathrm{FCH}_{2} \mathrm{C}(=\mathrm{O}) \mathrm{OH}$ & + & $\mathrm{CH}_{3} \mathrm{CH}_{3}$ & $\rightarrow$ & $\mathrm{CH}_{3} \mathrm{CH}_{2} \mathrm{C}(=\mathrm{O}) \mathrm{OH}$ & + & $\mathrm{CH}_{3} \mathrm{CH}_{2} \mathrm{~F}$ & -151.58 & -151.45 & -152.01 & -151.73 & -151.70 & -151.69 \\
\hline $\mathrm{CH}_{2} \mathrm{FCH}_{2} \mathrm{C}(=\mathrm{O}) \mathrm{OH}$ & + & $\mathrm{CH}_{3} \mathrm{CH}_{2} \mathrm{~F}$ & $\rightarrow$ & $\mathrm{CH}_{3} \mathrm{CH}_{2} \mathrm{C}(=\mathrm{O}) \mathrm{OH}$ & + & $\mathrm{CH}_{2} \mathrm{FCH}_{2} \mathrm{~F}$ & -151.58 & -151.67 & -152.64 & -151.63 & -151.57 & -151.52 \\
\hline $\mathrm{CH}_{2} \mathrm{FCH}_{2} \mathrm{C}(=\mathrm{O}) \mathrm{OH}$ & + & $\mathrm{CH}_{2} \mathrm{FCH}_{2} \mathrm{~F}$ & $\rightarrow$ & $\mathrm{CH}_{3} \mathrm{CH}_{2} \mathrm{C}(=\mathrm{O}) \mathrm{OH}$ & + & $\mathrm{CH}_{2} \mathrm{FCHF}_{2}$ & -151.63 & -153.18 & -150.46 & -151.87 & -151.43 & -151.75 \\
\hline $\mathrm{CH}_{2} \mathrm{FCH}_{2} \mathrm{C}(=\mathrm{O}) \mathrm{OH}$ & + & $\mathrm{CH}_{4}$ & $\rightarrow$ & $\mathrm{CH}_{3} \mathrm{CH}_{2} \mathrm{C}(=\mathrm{O}) \mathrm{OH}$ & + & $\mathrm{CH}_{3} \mathrm{~F}$ & -151.40 & -151.42 & -152.22 & -151.72 & -151.64 & -151.59 \\
\hline $\mathrm{CH}_{2} \mathrm{FCH}_{2} \mathrm{C}(=\mathrm{O}) \mathrm{OH}$ & + & $\mathrm{CH}_{3} \mathrm{CH}_{2} \mathrm{CH}_{3}$ & $\rightarrow$ & $\mathrm{CH}_{3} \mathrm{CH}_{2} \mathrm{C}(=\mathrm{O}) \mathrm{OH}$ & + & $\mathrm{CH}_{3} \mathrm{CH}_{2} \mathrm{CH}_{2} \mathrm{~F}$ & -151.61 & -151.81 & -151.98 & -151.86 & -151.97 & -151.93 \\
\hline $\mathrm{CH}_{2} \mathrm{FCH}_{2} \mathrm{C}(=\mathrm{O}) \mathrm{OH}$ & + & $\mathrm{CH}_{3} \mathrm{CH}_{2} \mathrm{CH}_{2} \mathrm{CH}_{3}$ & $\rightarrow$ & $\mathrm{CH}_{3} \mathrm{CH}_{2} \mathrm{C}(=\mathrm{O}) \mathrm{OH}$ & + & $\mathrm{CH}_{3} \mathrm{CH}_{2} \mathrm{CH}_{2} \mathrm{CH}_{2} \mathrm{~F}$ & -151.90 & -151.88 & -152.07 & -151.96 & -152.47 & -152.02 \\
\hline $\mathrm{CH}_{2} \mathrm{FCH}_{2} \mathrm{C}(=\mathrm{O}) \mathrm{OH}$ & + & $\mathrm{CH}_{3} \mathrm{CH}(=\mathrm{O})$ & $\rightarrow$ & $\mathrm{CH}_{3} \mathrm{CH}_{2} \mathrm{C}(=\mathrm{O}) \mathrm{OH}$ & + & $\mathrm{CH}_{2} \mathrm{FCH}(=\mathrm{O})$ & -152.29 & -152.48 & -152.61 & -152.46 & -152.46 & -152.47 \\
\hline $\mathrm{CH}_{2} \mathrm{FCH}_{2} \mathrm{C}(=\mathrm{O}) \mathrm{OH}$ & + & $\mathrm{CH}_{3} \mathrm{CH}(=\mathrm{O})$ & $\rightarrow$ & $\mathrm{CH}_{3} \mathrm{CH}_{2} \mathrm{C}(=\mathrm{O}) \mathrm{OH}$ & + & $\mathrm{CH}_{3} \mathrm{CF}(=\mathrm{O})$ & -152.81 & -153.79 & -151.89 & -152.76 & -151.95 & -152.61 \\
\hline $\mathrm{CH}_{2} \mathrm{FCH}_{2} \mathrm{C}(=\mathrm{O}) \mathrm{OH}$ & + & $\mathrm{CH}_{3} \mathrm{CH}_{2} \mathrm{CH}(=\mathrm{O})$ & $\rightarrow$ & $\mathrm{CH}_{3} \mathrm{CH}_{2} \mathrm{C}(=\mathrm{O}) \mathrm{OH}$ & + & $\mathrm{CH}_{2} \mathrm{FCH}_{2} \mathrm{CH}(=\mathrm{O})$ & -151.84 & -151.87 & -151.45 & -151.72 & -151.37 & -151.72 \\
\hline \multirow[t]{3}{*}{$\mathrm{CH}_{2} \mathrm{FCH}_{2} \mathrm{C}(=\mathrm{O}) \mathrm{OH}$} & + & $\mathrm{CH}_{3} \mathrm{C}(=\mathrm{O}) \mathrm{CH}_{3}$ & $\rightarrow$ & $\mathrm{CH}_{3} \mathrm{CH}_{2} \mathrm{C}(=\mathrm{O}) \mathrm{OH}$ & + & $\mathrm{CH}_{2} \mathrm{FC}(=\mathrm{O}) \mathrm{CH}_{3}$ & -152.99 & -153.27 & -152.47 & -153.35 & -152.86 & -152.86 \\
\hline & & & & & & Average & -151.96 & -152.28 & -151.98 & -152.11 & -151.94 & -152.02 \\
\hline & & & & & & Uncertainty & 0.88 & 1.35 & 0.91 & 0.94 & 0.78 & 0.86 \\
\hline
\end{tabular}




\begin{tabular}{|c|c|c|c|c|c|c|c|c|c|c|c|c|}
\hline \multirow{2}{*}{\multicolumn{7}{|c|}{ Isodesmic reactions }} & \multicolumn{6}{|c|}{$\Delta_{f} H^{\circ}{ }_{298}\left(\mathrm{kcal} \mathrm{mol}^{-1}\right)$} \\
\hline & & & & & & & \multirow[t]{2}{*}{ M06-2X } & \multirow[t]{2}{*}{ B3LYP } & \multirow[t]{2}{*}{ MN15 } & \multirow[t]{2}{*}{ CBS-QB3 } & \multirow[t]{2}{*}{ CBS-APNO } & \multirow[t]{2}{*}{ G4 } \\
\hline & & $\mathrm{H}_{2} \mathrm{FCHFC}(=0) \mathrm{O}$ & & & & & & & & & & \\
\hline $\mathrm{CH}_{2} \mathrm{FCHFC}(=\mathrm{O}) \mathrm{OH}$ & + & $\mathrm{CH}_{3} \mathrm{CHF}_{2}$ & $\rightarrow$ & $\mathrm{CH}_{3} \mathrm{CH}_{2} \mathrm{C}(=\mathrm{O}) \mathrm{OH}$ & + & $\mathrm{CH}_{2} \mathrm{FCF}_{3}$ & -194.93 & -196.93 & -194.33 & -194.97 & -193.97 & -194.56 \\
\hline $\mathrm{CH}_{2} \mathrm{FCHFC}(=\mathrm{O}) \mathrm{OH}$ & + & $\mathrm{CH}_{3} \mathrm{CH}_{3}$ & $\rightarrow$ & $\mathrm{CH}_{3} \mathrm{CH}_{2} \mathrm{C}(=\mathrm{O}) \mathrm{OH}$ & + & $\mathrm{CH}_{3} \mathrm{CHF}_{2}$ & -191.86 & -192.22 & -192.58 & -192.36 & -191.78 & -192.34 \\
\hline $\mathrm{CH}_{2} \mathrm{FCHFC}(=\mathrm{O}) \mathrm{OH}$ & + & $\mathrm{CH}_{3} \mathrm{CH}_{2} \mathrm{~F}$ & $\rightarrow$ & $\mathrm{CH}_{3} \mathrm{CH}_{2} \mathrm{C}(=\mathrm{O}) \mathrm{OH}$ & + & $\mathrm{CH}_{2} \mathrm{FCHF}_{2}$ & -191.74 & -192.48 & -191.99 & -192.09 & -191.52 & -191.98 \\
\hline $\mathrm{CH}_{2} \mathrm{FCHFC}(=\mathrm{O}) \mathrm{OH}$ & + & $\mathrm{CH}_{4}$ & $\rightarrow$ & $\mathrm{CH}_{3} \mathrm{CH}_{2} \mathrm{C}(=\mathrm{O}) \mathrm{OH}$ & + & $\mathrm{CH}_{2} \mathrm{~F}_{2}$ & -192.05 & -192.12 & -192.91 & -192.70 & -191.94 & -192.36 \\
\hline $\mathrm{CH}_{2} \mathrm{FCHFC}(=\mathrm{O}) \mathrm{OH}$ & + & $\mathrm{CH}_{3} \mathrm{CH}_{2} \mathrm{CH}_{3}$ & $\rightarrow$ & $\mathrm{CH}_{3} \mathrm{CH}_{2} \mathrm{C}(=\mathrm{O}) \mathrm{OH}$ & + & $\mathrm{CH}_{3} \mathrm{CH}_{2} \mathrm{CHF}_{2}$ & -192.41 & -193.11 & -193.10 & -193.03 & -192.60 & -193.08 \\
\hline $\mathrm{CH}_{2} \mathrm{FCHFC}(=\mathrm{O}) \mathrm{OH}$ & + & $\mathrm{CH}_{3} \mathrm{CH}_{2} \mathrm{CH}_{3}$ & $\rightarrow$ & $\mathrm{CH}_{3} \mathrm{CH}_{2} \mathrm{C}(=\mathrm{O}) \mathrm{OH}$ & + & $\mathrm{CH}_{3} \mathrm{CF}_{2} \mathrm{CH}_{3}$ & -192.30 & -193.50 & -193.46 & -192.92 & -192.61 & -193.14 \\
\hline $\mathrm{CH}_{2} \mathrm{FCHFC}(=\mathrm{O}) \mathrm{OH}$ & + & $\mathrm{CH}_{3} \mathrm{CH}(=\mathrm{O})$ & $\rightarrow$ & $\mathrm{CH}_{3} \mathrm{CH}_{2} \mathrm{C}(=\mathrm{O}) \mathrm{OH}$ & + & $\mathrm{CHF}_{2} \mathrm{CH}(=\mathrm{O})$ & -193.32 & -193.67 & -193.58 & -193.21 & -192.67 & -193.11 \\
\hline $\mathrm{CH}_{2} \mathrm{FCHFC}(=\mathrm{O}) \mathrm{OH}$ & + & $\mathrm{CH}_{3} \mathrm{CH}(=\mathrm{O})$ & $\rightarrow$ & $\mathrm{CH}_{3} \mathrm{CH}_{2} \mathrm{C}(=\mathrm{O}) \mathrm{OH}$ & + & $\mathrm{CH}_{2} \mathrm{FCF}(=\mathrm{O})$ & -193.92 & -194.20 & -193.00 & -193.71 & -192.79 & -193.61 \\
\hline $\mathrm{CH}_{2} \mathrm{FCHFC}(=\mathrm{O}) \mathrm{OH}$ & + & $\mathrm{CH}_{3} \mathrm{CH}_{2} \mathrm{CH}(=\mathrm{O})$ & $\rightarrow$ & $\mathrm{CH}_{3} \mathrm{CH}_{2} \mathrm{C}(=\mathrm{O}) \mathrm{OH}$ & + & $\mathrm{CHF}_{2} \mathrm{CH}_{2} \mathrm{CH}(=\mathrm{O})$ & -192.06 & -192.63 & -192.48 & -192.33 & -191.87 & -192.33 \\
\hline \multirow[t]{4}{*}{$\mathrm{CH}_{2} \mathrm{FCHFC}(=\mathrm{O}) \mathrm{OH}$} & + & $\mathrm{CH}_{3} \mathrm{C}(=\mathrm{O}) \mathrm{CH}_{3}$ & $\rightarrow$ & $\mathrm{CH}_{3} \mathrm{CH}_{2} \mathrm{C}(=\mathrm{O}) \mathrm{OH}$ & + & $\mathrm{CHF}_{2} \mathrm{C}(=\mathrm{O}) \mathrm{CH}_{3}$ & -193.31 & -194.47 & -193.50 & -194.01 & -193.10 & -193.49 \\
\hline & & & & & & Average & -192.79 & -193.53 & -193.09 & -193.13 & -192.48 & -193.00 \\
\hline & & & & & & Uncertainty & 0.70 & 1.22 & 0.74 & 0.77 & 0.60 & 0.68 \\
\hline & & $\mathrm{H}_{2} \mathrm{FCF}_{2} \mathrm{C}(=\mathrm{O}) \mathrm{OH}$ & & & & & & & & & & \\
\hline $\mathrm{CH}_{2} \mathrm{FCF}_{2} \mathrm{C}(=\mathrm{O}) \mathrm{OH}$ & + & $\mathrm{CH}_{3} \mathrm{CH}_{3}$ & $\rightarrow$ & $\mathrm{CH}_{3} \mathrm{CH}_{2} \mathrm{C}(=\mathrm{O}) \mathrm{OH}$ & + & $\mathrm{CH}_{2} \mathrm{FCHF}_{2}$ & -241.51 & -240.71 & -242.05 & -242.07 & -241.80 & -241.93 \\
\hline $\mathrm{CH}_{2} \mathrm{FCF}_{2} \mathrm{C}(=\mathrm{O}) \mathrm{OH}$ & + & $\mathrm{CH}_{3} \mathrm{CH}_{2} \mathrm{~F}$ & $\rightarrow$ & $\mathrm{CH}_{3} \mathrm{CH}_{2} \mathrm{C}(=\mathrm{O}) \mathrm{OH}$ & + & $\mathrm{CH}_{2} \mathrm{FCF}_{3}$ & -244.55 & -246.53 & -243.74 & -244.92 & -243.78 & -244.42 \\
\hline $\mathrm{CH}_{2} \mathrm{FCF}_{2} \mathrm{C}(=\mathrm{O}) \mathrm{OH}$ & + & $\mathrm{CH}_{3} \mathrm{CHF}_{2}$ & $\rightarrow$ & $\mathrm{CH}_{3} \mathrm{CH}_{2} \mathrm{C}(=\mathrm{O}) \mathrm{OH}$ & + & $\mathrm{CHF}_{2} \mathrm{CF}_{3}$ & -242.18 & -244.37 & -240.82 & -242.27 & -241.04 & -241.57 \\
\hline $\mathrm{CH}_{2} \mathrm{FCF}_{2} \mathrm{C}(=\mathrm{O}) \mathrm{OH}$ & + & $\mathrm{CH}_{4}$ & $\rightarrow$ & $\mathrm{CH}_{3} \mathrm{CH}_{2} \mathrm{C}(=\mathrm{O}) \mathrm{OH}$ & + & $\mathrm{CHF}_{3}$ & -242.35 & -243.02 & -242.01 & -243.22 & -241.62 & -242.31 \\
\hline $\mathrm{CH}_{2} \mathrm{FCF}_{2} \mathrm{C}(=\mathrm{O}) \mathrm{OH}$ & + & $\mathrm{CH}_{3} \mathrm{CH}_{3}$ & $\rightarrow$ & $\mathrm{CH}_{3} \mathrm{CH}_{2} \mathrm{C}(=\mathrm{O}) \mathrm{OH}$ & + & $\mathrm{CH}_{3} \mathrm{CF}_{3}$ & -240.39 & -241.90 & -240.29 & -241.10 & -239.99 & -240.74 \\
\hline $\mathrm{CH}_{2} \mathrm{FCF}_{2} \mathrm{C}(=\mathrm{O}) \mathrm{OH}$ & + & $\mathrm{CH}_{3} \mathrm{CH}_{2} \mathrm{CH}_{3}$ & $\rightarrow$ & $\mathrm{CH}_{3} \mathrm{CH}_{2} \mathrm{C}(=\mathrm{O}) \mathrm{OH}$ & + & $\mathrm{CH}_{3} \mathrm{CH}_{2} \mathrm{CF}_{3}$ & -242.50 & -244.39 & -242.20 & -243.11 & -242.30 & -242.90 \\
\hline $\mathrm{CH}_{2} \mathrm{FCF}_{2} \mathrm{C}(=\mathrm{O}) \mathrm{OH}$ & + & $\mathrm{CH}_{3} \mathrm{CH}(=\mathrm{O})$ & $\rightarrow$ & $\mathrm{CH}_{3} \mathrm{CH}_{2} \mathrm{C}(=\mathrm{O}) \mathrm{OH}$ & + & $\mathrm{CF}_{3} \mathrm{CH}(=\mathrm{O})$ & -243.40 & -244.85 & -242.38 & -243.29 & -242.08 & -242.66 \\
\hline $\mathrm{CH}_{2} \mathrm{FCF}_{2} \mathrm{C}(=\mathrm{O}) \mathrm{OH}$ & + & $\mathrm{CH}_{3} \mathrm{CH}(=\mathrm{O})$ & $\rightarrow$ & $\mathrm{CH}_{3} \mathrm{CH}_{2} \mathrm{C}(=\mathrm{O}) \mathrm{OH}$ & + & $\mathrm{CHF}_{2} \mathrm{CF}(=\mathrm{O})$ & -243.95 & -244.00 & -242.44 & -243.54 & -242.43 & -243.18 \\
\hline $\mathrm{CH}_{2} \mathrm{FCF}_{2} \mathrm{C}(=\mathrm{O}) \mathrm{OH}$ & + & $\mathrm{CH}_{3} \mathrm{CH}_{2} \mathrm{CH}(=\mathrm{O})$ & $\rightarrow$ & $\mathrm{CH}_{3} \mathrm{CH}_{2} \mathrm{C}(=\mathrm{O}) \mathrm{OH}$ & + & $\mathrm{CF}_{3} \mathrm{CH}_{2} \mathrm{CH}(=\mathrm{O})$ & -242.22 & -243.76 & -241.90 & -242.43 & -241.49 & -242.14 \\
\hline $\mathrm{CH}_{2} \mathrm{FCF}_{2} \mathrm{C}(=\mathrm{O}) \mathrm{OH}$ & + & $\mathrm{CH}_{3} \mathrm{CH}_{2} \mathrm{CH}(=\mathrm{O})$ & $\rightarrow$ & $\mathrm{CH}_{3} \mathrm{CH}_{2} \mathrm{C}(=\mathrm{O}) \mathrm{OH}$ & + & $\mathrm{CHF}_{2} \mathrm{CHFCH}(=\mathrm{O})$ & -242.74 & -242.52 & -242.91 & -242.73 & -242.63 & -242.72 \\
\hline \multirow[t]{3}{*}{$\mathrm{CH}_{2} \mathrm{FCF}_{2} \mathrm{C}(=\mathrm{O}) \mathrm{OH}$} & + & $\mathrm{CH}_{3} \mathrm{C}(=\mathrm{O}) \mathrm{CH}_{3}$ & $\rightarrow$ & $\mathrm{CH}_{3} \mathrm{CH}_{2} \mathrm{C}(=\mathrm{O}) \mathrm{OH}$ & + & $\mathrm{CF}_{3} \mathrm{C}(=\mathrm{O}) \mathrm{CH}_{3}$ & -243.81 & -245.53 & -242.20 & -243.93 & -242.46 & -242.93 \\
\hline & & & & & & Average & -242.69 & -243.78 & -242.08 & -242.96 & -241.97 & -242.50 \\
\hline & & & & & & Uncertainty & 0.78 & 1.27 & 0.81 & 0.84 & 0.69 & 0.76 \\
\hline
\end{tabular}




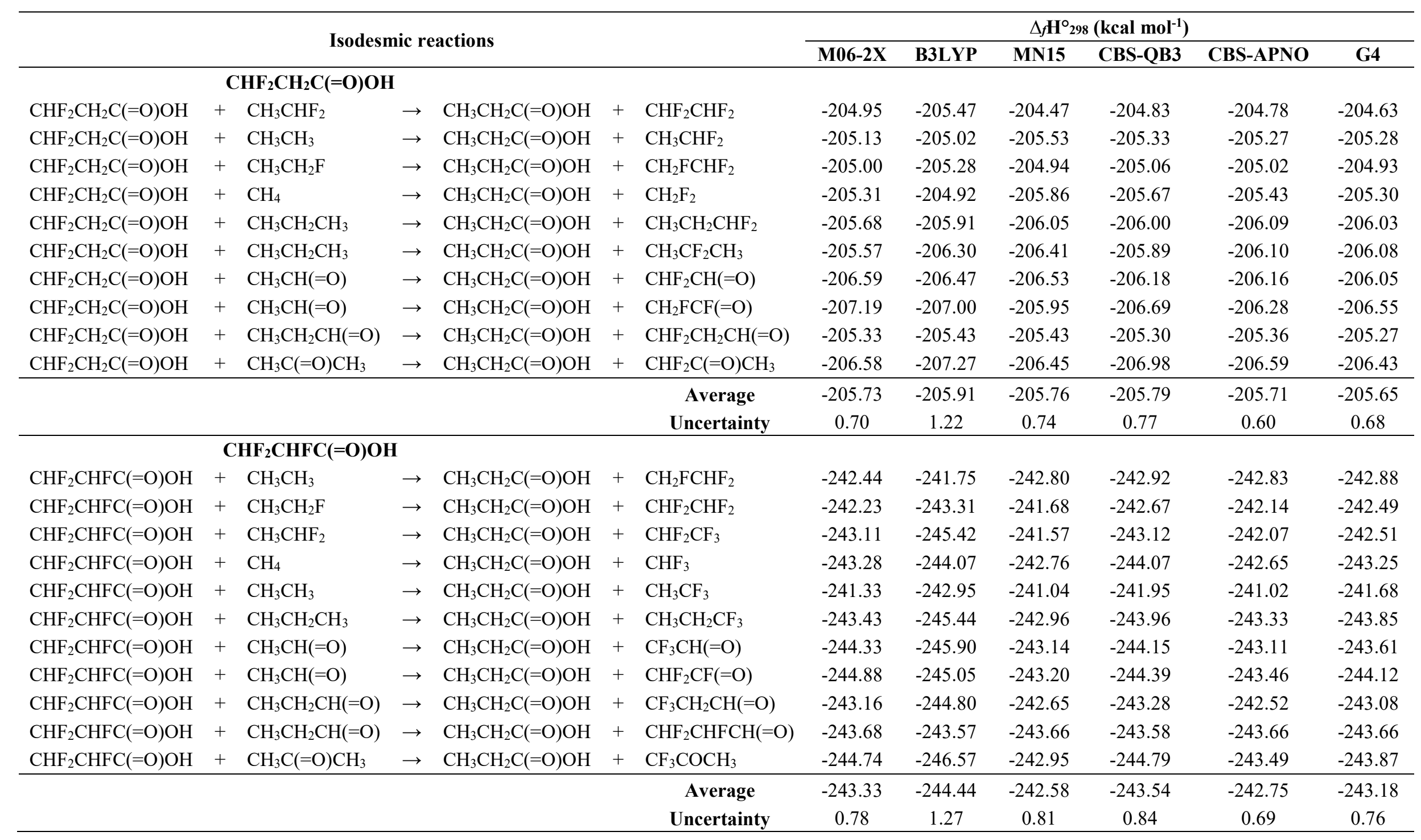




\begin{tabular}{|c|c|c|c|c|c|c|c|c|c|c|c|c|}
\hline \multirow{2}{*}{\multicolumn{7}{|c|}{ Isodesmic reactions }} & \multicolumn{6}{|c|}{$\Delta_{f} \mathbf{H}^{\circ}{ }_{298}\left(\mathrm{kcal} \mathrm{mol}^{-1}\right)$} \\
\hline & & & & & & & \multirow[t]{2}{*}{ M06-2X } & \multirow[t]{2}{*}{ B3LYP } & \multirow[t]{2}{*}{ MN15 } & \multirow[t]{2}{*}{ CBS-QB3 } & \multirow[t]{2}{*}{ CBS-APNO } & \multirow[t]{2}{*}{ G4 } \\
\hline & & $\mathrm{CHF}_{2} \mathrm{CF}_{2} \mathrm{C}(=\mathrm{O}) \mathrm{OH}$ & & & & & & & & & & \\
\hline $\mathrm{CHF}_{2} \mathrm{CF}_{2} \mathrm{C}(=\mathrm{O}) \mathrm{OH}$ & + & $\mathrm{CH}_{2} \mathrm{FCH}_{2} \mathrm{~F}$ & $\rightarrow$ & $\mathrm{CH}_{3} \mathrm{CH}_{2} \mathrm{C}(=\mathrm{O}) \mathrm{OH}$ & + & $\mathrm{CF}_{3} \mathrm{CF}_{3}$ & -292.08 & -296.48 & -289.10 & -292.25 & -289.99 & -291.18 \\
\hline $\mathrm{CHF}_{2} \mathrm{CF}_{2} \mathrm{C}(=\mathrm{O}) \mathrm{OH}$ & + & $\mathrm{CH}_{3} \mathrm{CH}_{3}$ & $\rightarrow$ & $\mathrm{CH}_{3} \mathrm{CH}_{2} \mathrm{C}(=\mathrm{O}) \mathrm{OH}$ & + & $\mathrm{CHF}_{2} \mathrm{CHF}_{2}$ & -290.25 & -289.46 & -291.01 & -290.99 & -290.70 & -290.85 \\
\hline $\mathrm{CHF}_{2} \mathrm{CF}_{2} \mathrm{C}(=\mathrm{O}) \mathrm{OH}$ & + & $\mathrm{CH}_{3} \mathrm{CH}_{2} \mathrm{~F}$ & $\rightarrow$ & $\mathrm{CH}_{3} \mathrm{CH}_{2} \mathrm{C}(=\mathrm{O}) \mathrm{OH}$ & + & $\mathrm{CHF}_{2} \mathrm{CF}_{3}$ & -290.98 & -292.94 & -290.25 & -291.43 & -290.18 & -290.79 \\
\hline $\mathrm{CHF}_{2} \mathrm{CF}_{2} \mathrm{C}(=\mathrm{O}) \mathrm{OH}$ & + & $\mathrm{CH}_{4}$ & $\rightarrow$ & $\mathrm{CH}_{3} \mathrm{CH}_{2} \mathrm{C}(=\mathrm{O}) \mathrm{OH}$ & + & $\mathrm{CF}_{4}$ & -292.18 & -294.27 & -291.02 & -293.14 & -290.66 & -291.76 \\
\hline $\mathrm{CHF}_{2} \mathrm{CF}_{2} \mathrm{C}(=\mathrm{O}) \mathrm{OH}$ & + & $\mathrm{CH}_{3} \mathrm{CH}_{3}$ & $\rightarrow$ & $\mathrm{CH}_{3} \mathrm{CH}_{2} \mathrm{C}(=\mathrm{O}) \mathrm{OH}$ & + & $\mathrm{CH}_{2} \mathrm{FCF}_{3}$ & -293.51 & -293.73 & -293.82 & -294.10 & -293.38 & -293.73 \\
\hline $\mathrm{CHF}_{2} \mathrm{CF}_{2} \mathrm{C}(=\mathrm{O}) \mathrm{OH}$ & + & $\mathrm{CH}_{3} \mathrm{CH}(=\mathrm{O})$ & $\rightarrow$ & $\mathrm{CH}_{3} \mathrm{CH}_{2} \mathrm{C}(=\mathrm{O}) \mathrm{OH}$ & + & $\mathrm{CF}_{3} \mathrm{CF}(=\mathrm{O})$ & -294.39 & -295.31 & -292.34 & -293.97 & -292.31 & -293.25 \\
\hline $\mathrm{CHF}_{2} \mathrm{CF}_{2} \mathrm{C}(=\mathrm{O}) \mathrm{OH}$ & + & $\mathrm{CH}_{3} \mathrm{CH}_{2} \mathrm{CH}(=\mathrm{O})$ & $\rightarrow$ & $\mathrm{CH}_{3} \mathrm{CH}_{2} \mathrm{C}(=\mathrm{O}) \mathrm{OH}$ & + & $\mathrm{CF}_{3} \mathrm{CHFCH}(=\mathrm{O})$ & -291.95 & -292.68 & -291.93 & -291.77 & -291.35 & -291.65 \\
\hline $\mathrm{CHF}_{2} \mathrm{CF}_{2} \mathrm{C}(=\mathrm{O}) \mathrm{OH}$ & + & $\mathrm{CH}_{3} \mathrm{CH}_{2} \mathrm{CH}(=\mathrm{O})$ & $\rightarrow$ & $\mathrm{CH}_{3} \mathrm{CH}_{2} \mathrm{C}(=\mathrm{O}) \mathrm{OH}$ & + & $\mathrm{CF}_{3} \mathrm{CH}_{2} \mathrm{CF}(=\mathrm{O})$ & -292.43 & -293.49 & -291.33 & -292.35 & -291.11 & -292.02 \\
\hline $\mathrm{CHF}_{2} \mathrm{CF}_{2} \mathrm{C}(=\mathrm{O}) \mathrm{OH}$ & + & $\mathrm{CH}_{3} \mathrm{C}(=\mathrm{O}) \mathrm{CH}_{3}$ & $\rightarrow$ & $\mathrm{CH}_{3} \mathrm{CH}_{2} \mathrm{C}(=\mathrm{O}) \mathrm{OH}$ & + & $\mathrm{CF}_{3} \mathrm{C}(=\mathrm{O}) \mathrm{CH}_{2} \mathrm{~F}$ & -292.92 & -293.73 & -292.17 & -293.44 & -292.33 & -292.53 \\
\hline \multirow[t]{4}{*}{$\mathrm{CHF}_{2} \mathrm{CF}_{2} \mathrm{C}(=\mathrm{O}) \mathrm{OH}$} & + & $\mathrm{CH}_{3} \mathrm{C}(=\mathrm{O}) \mathrm{CH}_{3}$ & $\rightarrow$ & $\mathrm{CH}_{3} \mathrm{CH}_{2} \mathrm{C}(=\mathrm{O}) \mathrm{OH}$ & + & $\mathrm{CHF}_{2} \mathrm{C}(=\mathrm{O}) \mathrm{CHF}_{2}$ & -293.28 & -292.94 & -293.31 & -293.60 & -293.17 & -292.85 \\
\hline & & & & & & Average & -292.40 & -293.50 & -291.63 & -292.70 & -291.52 & -292.06 \\
\hline & & & & & & Uncertainty & 0.95 & 1.40 & 0.98 & 1.01 & 0.87 & 0.93 \\
\hline & & $\mathrm{CF}_{3} \mathrm{CH}_{2} \mathrm{C}(=\mathrm{O}) \mathrm{OH}$ & & & & & & & & & & \\
\hline $\mathrm{CF}_{3} \mathrm{CH}_{2} \mathrm{C}(=\mathrm{O}) \mathrm{OH}$ & + & $\mathrm{CH}_{3} \mathrm{CH}_{3}$ & $\rightarrow$ & $\mathrm{CH}_{3} \mathrm{CH}_{2} \mathrm{C}(=\mathrm{O}) \mathrm{OH}$ & + & $\mathrm{CH}_{3} \mathrm{CF}_{3}$ & -260.07 & -259.71 & -260.67 & -260.51 & -260.30 & -260.50 \\
\hline $\mathrm{CF}_{3} \mathrm{CH}_{2} \mathrm{C}(=\mathrm{O}) \mathrm{OH}$ & + & $\mathrm{CH}_{3} \mathrm{CH}_{2} \mathrm{~F}$ & $\rightarrow$ & $\mathrm{CH}_{3} \mathrm{CH}_{2} \mathrm{C}(=\mathrm{O}) \mathrm{OH}$ & + & $\mathrm{CH}_{2} \mathrm{FCF}_{3}$ & -264.23 & -264.34 & -264.12 & -264.34 & -264.09 & -264.18 \\
\hline $\mathrm{CF}_{3} \mathrm{CH}_{2} \mathrm{C}(=\mathrm{O}) \mathrm{OH}$ & + & $\mathrm{CH}_{3} \mathrm{CHF}_{2}$ & $\rightarrow$ & $\mathrm{CH}_{3} \mathrm{CH}_{2} \mathrm{C}(=\mathrm{O}) \mathrm{OH}$ & + & $\mathrm{CHF}_{2} \mathrm{CF}_{3}$ & -261.85 & -262.19 & -261.20 & -261.68 & -261.35 & -261.33 \\
\hline $\mathrm{CF}_{3} \mathrm{CH}_{2} \mathrm{C}(=\mathrm{O}) \mathrm{OH}$ & + & $\mathrm{CH}_{4}$ & $\rightarrow$ & $\mathrm{CH}_{3} \mathrm{CH}_{2} \mathrm{C}(=\mathrm{O}) \mathrm{OH}$ & + & $\mathrm{CHF}_{3}$ & -262.02 & -260.83 & -262.39 & -262.63 & -261.93 & -262.06 \\
\hline $\mathrm{CF}_{3} \mathrm{CH}_{2} \mathrm{C}(=\mathrm{O}) \mathrm{OH}$ & + & $\mathrm{CH}_{3} \mathrm{CH}_{2} \mathrm{CH}_{3}$ & $\rightarrow$ & $\mathrm{CH}_{3} \mathrm{CH}_{2} \mathrm{C}(=\mathrm{O}) \mathrm{OH}$ & + & $\mathrm{CH}_{3} \mathrm{CH}_{2} \mathrm{CF}_{3}$ & -262.17 & -262.20 & -262.58 & -262.52 & -262.61 & -262.66 \\
\hline $\mathrm{CF}_{3} \mathrm{CH}_{2} \mathrm{C}(=\mathrm{O}) \mathrm{OH}$ & + & $\mathrm{CH}_{3} \mathrm{CH}(=\mathrm{O})$ & $\rightarrow$ & $\mathrm{CH}_{3} \mathrm{CH}_{2} \mathrm{C}(=\mathrm{O}) \mathrm{OH}$ & + & $\mathrm{CF}_{3} \mathrm{CH}(=\mathrm{O})$ & -263.08 & -262.67 & -262.77 & -262.71 & -262.39 & -262.42 \\
\hline $\mathrm{CF}_{3} \mathrm{CH}_{2} \mathrm{C}(=\mathrm{O}) \mathrm{OH}$ & + & $\mathrm{CH}_{3} \mathrm{CH}(=\mathrm{O})$ & $\rightarrow$ & $\mathrm{CH}_{3} \mathrm{CH}_{2} \mathrm{C}(=\mathrm{O}) \mathrm{OH}$ & + & $\mathrm{CHF}_{2} \mathrm{CF}(=\mathrm{O})$ & -263.63 & -261.81 & -262.83 & -262.95 & -262.74 & -262.93 \\
\hline $\mathrm{CF}_{3} \mathrm{CH}_{2} \mathrm{C}(=\mathrm{O}) \mathrm{OH}$ & + & $\mathrm{CH}_{3} \mathrm{CH}_{2} \mathrm{CH}(=\mathrm{O})$ & $\rightarrow$ & $\mathrm{CH}_{3} \mathrm{CH}_{2} \mathrm{C}(=\mathrm{O}) \mathrm{OH}$ & + & $\mathrm{CF}_{3} \mathrm{CH}_{2} \mathrm{CH}(=\mathrm{O})$ & -261.90 & -261.57 & -262.28 & -261.84 & -261.80 & -261.89 \\
\hline $\mathrm{CF}_{3} \mathrm{CH}_{2} \mathrm{C}(=\mathrm{O}) \mathrm{OH}$ & + & $\mathrm{CH}_{3} \mathrm{CH}_{2} \mathrm{CH}(=\mathrm{O})$ & $\rightarrow$ & $\mathrm{CH}_{3} \mathrm{CH}_{2} \mathrm{C}(=\mathrm{O}) \mathrm{OH}$ & + & $\mathrm{CHF}_{2} \mathrm{CHFCH}(=\mathrm{O})$ & -262.42 & -260.34 & -263.29 & -262.14 & -262.94 & -262.47 \\
\hline \multirow[t]{3}{*}{$\mathrm{CF}_{3} \mathrm{CH}_{2} \mathrm{C}(=\mathrm{O}) \mathrm{OH}$} & + & $\mathrm{CH}_{3} \mathrm{C}(=\mathrm{O}) \mathrm{CH}_{3}$ & $\rightarrow$ & $\mathrm{CH}_{3} \mathrm{CH}_{2} \mathrm{C}(=\mathrm{O}) \mathrm{OH}$ & + & $\mathrm{CF}_{3} \mathrm{C}(=\mathrm{O}) \mathrm{CH}_{3}$ & -263.49 & -263.34 & -262.58 & -263.35 & -262.77 & -262.68 \\
\hline & & & & & & Average & -262.49 & -261.90 & -262.47 & -262.47 & -262.29 & -262.31 \\
\hline & & & & & & Uncertainty & 0.77 & 1.27 & 0.80 & 0.83 & 0.67 & 0.75 \\
\hline
\end{tabular}




\begin{tabular}{|c|c|c|c|c|c|c|c|c|c|c|c|c|}
\hline \multirow{2}{*}{\multicolumn{7}{|c|}{ Isodesmic reactions }} & \multicolumn{6}{|c|}{$\Delta_{f} \mathbf{H}^{\circ}{ }_{298}\left(\mathrm{kcal} \mathrm{mol}^{-1}\right)$} \\
\hline & & & & & & & \multirow[t]{2}{*}{ M06-2X } & \multirow[t]{2}{*}{ B3LYP } & \multirow[t]{2}{*}{ MN15 } & \multirow[t]{2}{*}{ CBS-QB3 } & \multirow[t]{2}{*}{$\begin{array}{c}\text { CBS- } \\
\text { APNO }\end{array}$} & \multirow[t]{2}{*}{ G4 } \\
\hline & & $\mathrm{CF}_{3} \mathrm{CHFC}(=\mathrm{O}) \mathrm{O}$ & & & & & & & & & & \\
\hline $\mathrm{CF}_{3} \mathrm{CHFC}(=\mathrm{O}) \mathrm{OH}$ & + & $\mathrm{CH}_{3} \mathrm{CHF}_{2}$ & $\rightarrow$ & $\mathrm{CH}_{3} \mathrm{CH}_{2} \mathrm{C}(=\mathrm{O}) \mathrm{OH}$ & + & $\mathrm{CF}_{3} \mathrm{CF}_{3}$ & -299.34 & -301.49 & -297.89 & -299.29 & -297.95 & -298.55 \\
\hline $\mathrm{CF}_{3} \mathrm{CHFC}(=\mathrm{O}) \mathrm{OH}$ & + & $\mathrm{CH}_{3} \mathrm{CH}_{3}$ & $\rightarrow$ & $\mathrm{CH}_{3} \mathrm{CH}_{2} \mathrm{C}(=\mathrm{O}) \mathrm{OH}$ & + & $\mathrm{CH}_{2} \mathrm{FCF}_{3}$ & -300.61 & -299.87 & -301.33 & -301.21 & -301.00 & -301.18 \\
\hline $\mathrm{CF}_{3} \mathrm{CHFC}(=\mathrm{O}) \mathrm{OH}$ & + & $\mathrm{CH}_{3} \mathrm{CH}_{2} \mathrm{~F}$ & $\rightarrow$ & $\mathrm{CH}_{3} \mathrm{CH}_{2} \mathrm{C}(=\mathrm{O}) \mathrm{OH}$ & + & $\mathrm{CHF}_{2} \mathrm{CF}_{3}$ & -298.08 & -299.09 & -297.75 & -298.54 & -297.79 & -298.24 \\
\hline $\mathrm{CF}_{3} \mathrm{CHFC}(=\mathrm{O}) \mathrm{OH}$ & + & $\mathrm{CH}_{4}$ & $\rightarrow$ & $\mathrm{CH}_{3} \mathrm{CH}_{2} \mathrm{C}(=\mathrm{O}) \mathrm{OH}$ & + & $\mathrm{CF}_{4}$ & -299.28 & -300.41 & -298.52 & -300.25 & -298.27 & -299.21 \\
\hline $\mathrm{CF}_{3} \mathrm{CHFC}(=\mathrm{O}) \mathrm{OH}$ & + & $\mathrm{CH}_{3} \mathrm{CH}_{3}$ & $\rightarrow$ & $\mathrm{CH}_{3} \mathrm{CH}_{2} \mathrm{C}(=\mathrm{O}) \mathrm{OH}$ & + & $\mathrm{CHF}_{2} \mathrm{CHF}_{2}$ & -297.35 & -295.61 & -298.51 & -298.11 & -298.32 & -298.30 \\
\hline $\mathrm{CF}_{3} \mathrm{CHFC}(=\mathrm{O}) \mathrm{OH}$ & + & $\mathrm{CH}_{3} \mathrm{CH}(=\mathrm{O})$ & $\rightarrow$ & $\mathrm{CH}_{3} \mathrm{CH}_{2} \mathrm{C}(=\mathrm{O}) \mathrm{OH}$ & + & $\mathrm{CF}_{3} \mathrm{CF}(=\mathrm{O})$ & -301.49 & -301.46 & -299.85 & -301.08 & -299.92 & -300.70 \\
\hline $\mathrm{CF}_{3} \mathrm{CHFC}(=\mathrm{O}) \mathrm{OH}$ & + & $\mathrm{CH}_{3} \mathrm{CH}_{2} \mathrm{CH}(=\mathrm{O})$ & $\rightarrow$ & $\mathrm{CH}_{3} \mathrm{CH}_{2} \mathrm{C}(=\mathrm{O}) \mathrm{OH}$ & + & $\mathrm{CF}_{3} \mathrm{CHFCH}(=\mathrm{O})$ & -299.05 & -298.82 & -299.44 & -298.88 & -298.96 & -299.10 \\
\hline $\mathrm{CF}_{3} \mathrm{CHFC}(=\mathrm{O}) \mathrm{OH}$ & + & $\mathrm{CH}_{3} \mathrm{CH}_{2} \mathrm{CH}(=\mathrm{O})$ & $\rightarrow$ & $\mathrm{CH}_{3} \mathrm{CH}_{2} \mathrm{C}(=\mathrm{O}) \mathrm{OH}$ & + & $\mathrm{CF}_{3} \mathrm{CH}_{2} \mathrm{CF}(=\mathrm{O})$ & -299.53 & -299.64 & -298.84 & -299.46 & -298.72 & -299.47 \\
\hline $\mathrm{CF}_{3} \mathrm{CHFC}(=\mathrm{O}) \mathrm{OH}$ & + & $\mathrm{CH}_{3} \mathrm{C}(=\mathrm{O}) \mathrm{CH}_{3}$ & $\rightarrow$ & $\mathrm{CH}_{3} \mathrm{CH}_{2} \mathrm{C}(=\mathrm{O}) \mathrm{OH}$ & + & $\mathrm{CF}_{3} \mathrm{C}(=\mathrm{O}) \mathrm{CH}_{2} \mathrm{~F}$ & -300.03 & -299.87 & -299.67 & -300.55 & -299.94 & -299.97 \\
\hline \multirow[t]{4}{*}{$\mathrm{CF}_{3} \mathrm{CHFC}(=\mathrm{O}) \mathrm{OH}$} & + & $\mathrm{CH}_{3} \mathrm{C}(=\mathrm{O}) \mathrm{CH}_{3}$ & $\rightarrow$ & $\mathrm{CH}_{3} \mathrm{CH}_{2} \mathrm{C}(=\mathrm{O}) \mathrm{OH}$ & + & $\mathrm{CHF}_{2} \mathrm{C}(=\mathrm{O}) \mathrm{CHF}_{2}$ & -300.38 & -299.09 & -300.82 & -300.71 & -300.79 & -300.30 \\
\hline & & & & & & Average & -299.51 & -299.54 & -299.26 & -299.81 & -299.17 & -299.50 \\
\hline & & & & & & Uncertainty & 0.96 & 1.40 & 0.99 & 1.02 & 0.88 & 0.94 \\
\hline & & $\mathrm{CF}_{3} \mathrm{CF}_{2} \mathrm{C}(=\mathrm{O}) \mathrm{OH}$ & & & & & & & & & & \\
\hline $\mathrm{CF}_{3} \mathrm{CF}_{2} \mathrm{C}(=\mathrm{O}) \mathrm{OH}$ & + & $\mathrm{CH}_{3} \mathrm{CH}_{3}$ & $\rightarrow$ & $\mathrm{CH}_{3} \mathrm{CH}_{2} \mathrm{C}(=\mathrm{O}) \mathrm{OH}$ & + & $\mathrm{CHF}_{2} \mathrm{CF}_{3}$ & -344.66 & -343.78 & -345.58 & -345.59 & -345.13 & -345.35 \\
\hline $\mathrm{CF}_{3} \mathrm{CF}_{2} \mathrm{C}(=\mathrm{O}) \mathrm{OH}$ & + & $\mathrm{CH}_{3} \mathrm{CH}_{2} \mathrm{~F}$ & $\rightarrow$ & $\mathrm{CH}_{3} \mathrm{CH}_{2} \mathrm{C}(=\mathrm{O}) \mathrm{OH}$ & + & $\mathrm{CF}_{3} \mathrm{CF}_{3}$ & -345.76 & -347.55 & -345.06 & -346.32 & -344.82 & -345.57 \\
\hline $\mathrm{CF}_{3} \mathrm{CF}_{2} \mathrm{C}(=\mathrm{O}) \mathrm{OH}$ & + & $\mathrm{CH}_{3} \mathrm{~F}$ & $\rightarrow$ & $\mathrm{CH}_{3} \mathrm{C}(=\mathrm{O}) \mathrm{OH}$ & + & $\mathrm{CF}_{3} \mathrm{CF}_{3}$ & -345.74 & -346.51 & -345.02 & -346.55 & -345.05 & -345.81 \\
\hline $\mathrm{CF}_{3} \mathrm{CF}_{2} \mathrm{C}(=\mathrm{O}) \mathrm{OH}$ & + & $\mathrm{CH}_{3} \mathrm{CH}_{2} \mathrm{CH}(=\mathrm{O})$ & $\rightarrow$ & $\mathrm{CH}_{3} \mathrm{CH}_{2} \mathrm{C}(=\mathrm{O}) \mathrm{OH}$ & + & $\mathrm{CF}_{3} \mathrm{CF}_{2} \mathrm{CH}(=\mathrm{O})$ & -346.22 & -346.20 & -346.79 & -346.23 & -346.27 & -346.27 \\
\hline $\mathrm{CF}_{3} \mathrm{CF}_{2} \mathrm{C}(=\mathrm{O}) \mathrm{OH}$ & + & $\mathrm{CH}_{3} \mathrm{CH}_{2} \mathrm{CH}(=\mathrm{O})$ & $\rightarrow$ & $\mathrm{CH}_{3} \mathrm{CH}_{2} \mathrm{C}(=\mathrm{O}) \mathrm{OH}$ & + & $\mathrm{CF}_{3} \mathrm{CHFCF}(=\mathrm{O})$ & -347.10 & -346.15 & -346.52 & -346.77 & -346.32 & -346.79 \\
\hline $\mathrm{CF}_{3} \mathrm{CF}_{2} \mathrm{C}(=\mathrm{O}) \mathrm{OH}$ & + & $\mathrm{CH}_{3} \mathrm{CH}_{2} \mathrm{CH}(=\mathrm{O})$ & $\rightarrow$ & $\mathrm{CH}_{3} \mathrm{CH}_{2} \mathrm{C}(=\mathrm{O}) \mathrm{OH}$ & + & $\mathrm{CHF}_{2} \mathrm{CF}_{2} \mathrm{CF}(=\mathrm{O})$ & -346.15 & -344.53 & -346.26 & -345.80 & -346.08 & -346.21 \\
\hline \multirow[t]{3}{*}{$\mathrm{CF}_{3} \mathrm{CF}_{2} \mathrm{C}(=\mathrm{O}) \mathrm{OH}$} & + & $\mathrm{CH}_{3} \mathrm{C}(=\mathrm{O}) \mathrm{CH}_{3}$ & $\rightarrow$ & $\mathrm{CH}_{3} \mathrm{CH}_{2} \mathrm{C}(=\mathrm{O}) \mathrm{OH}$ & + & $\mathrm{CF}_{3} \mathrm{C}(=\mathrm{O}) \mathrm{CHF}_{2}$ & -348.44 & -347.20 & -347.72 & -348.16 & -347.44 & -347.55 \\
\hline & & & & & & Average & -346.30 & -345.99 & -346.14 & -346.49 & -345.87 & -346.22 \\
\hline & & & & & & Uncertainty & 1.10 & 1.53 & 1.12 & 1.15 & 1.01 & 1.08 \\
\hline
\end{tabular}




\section{Work reactions for florinated carboxylic acid radicals}

In this work, there are 36 species; 7 species for fluorinated acetic acid radical and 29 species for fluorinated propionic acid radical. The uncertainty is determined from the square root of the sum of squares of the uncertainty in the reference species and the uncertainty of each calculation method

\begin{tabular}{|c|c|c|c|c|c|c|c|c|c|c|c|c|}
\hline \multirow{2}{*}{\multicolumn{7}{|c|}{ Isodesmic reactions }} & \multicolumn{6}{|c|}{$\Delta_{f} \mathbf{H}^{\circ}{ }_{298}\left(\mathrm{kcal} \mathrm{mol}^{-1}\right)$} \\
\hline & & & & & & & \multirow[t]{2}{*}{ M06-2X } & \multirow[t]{2}{*}{ B3LYP } & \multirow[t]{2}{*}{ MN15 } & \multirow[t]{2}{*}{ CBS-QB3 } & \multirow[t]{2}{*}{ CBS-APNO } & \multirow[t]{2}{*}{ G4 } \\
\hline & & $\mathrm{CH}_{2} \cdot \mathrm{C}(=\mathrm{O}) \mathrm{O}$ & & & & & & & & & & \\
\hline $\mathrm{CH}_{2} \cdot \mathrm{C}(=\mathrm{O}) \mathrm{OH}$ & + & $\mathrm{CH}_{3} \mathrm{OH}$ & $\rightarrow$ & $\mathrm{CH}_{3} \mathrm{C}(=\mathrm{O}) \mathrm{OH}$ & + & $\mathrm{CH}_{2} \bullet \mathrm{OH}$ & -57.41 & -57.71 & -56.29 & -56.90 & -57.61 & -57.53 \\
\hline $\mathrm{CH}_{2} \cdot \mathrm{C}(=\mathrm{O}) \mathrm{OH}$ & + & $\mathrm{CH}_{3} \mathrm{CH}_{2} \mathrm{OH}$ & $\rightarrow$ & $\mathrm{CH}_{3} \mathrm{C}(=\mathrm{O}) \mathrm{OH}$ & + & $\mathrm{CH}_{2} \cdot \mathrm{CH}_{2} \mathrm{OH}$ & -57.14 & -57.40 & -56.90 & -57.18 & -57.24 & -57.10 \\
\hline $\mathrm{CH}_{2} \cdot \mathrm{C}(=\mathrm{O}) \mathrm{OH}$ & + & $\mathrm{CH}_{3} \mathrm{CH}(=\mathrm{O})$ & $\rightarrow$ & $\mathrm{CH}_{3} \mathrm{C}(=\mathrm{O}) \mathrm{OH}$ & + & $\mathrm{CH}_{2} \cdot \mathrm{CH}(=\mathrm{O})$ & -55.92 & -55.68 & -55.82 & -55.67 & -55.92 & -56.51 \\
\hline $\mathrm{CH}_{2} \cdot \mathrm{C}(=\mathrm{O}) \mathrm{OH}$ & + & $\mathrm{CH}_{3} \mathrm{CH}_{2} \mathrm{~F}$ & $\rightarrow$ & $\mathrm{CH}_{2} \mathrm{FC}(=\mathrm{O}) \mathrm{OH}$ & + & $\mathrm{CH}_{3} \mathrm{CH}_{2} \bullet$ & -58.03 & -58.86 & -57.28 & -57.76 & -57.80 & -57.93 \\
\hline $\mathrm{CH}_{2} \cdot \mathrm{C}(=\mathrm{O}) \mathrm{OH}$ & + & $\mathrm{CH}_{3} \mathrm{CHF}_{2}$ & $\rightarrow$ & $\mathrm{CH}_{2} \mathrm{FC}(=\mathrm{O}) \mathrm{OH}$ & + & $\mathrm{CH}_{3} \mathrm{CHF} \bullet$ & -57.59 & -56.86 & -56.18 & -57.44 & -57.63 & -57.56 \\
\hline $\mathrm{CH}_{2} \cdot \mathrm{C}(=\mathrm{O}) \mathrm{OH}$ & + & $\mathrm{CH}_{2} \mathrm{FCH}_{2} \mathrm{~F}$ & $\rightarrow$ & $\mathrm{CH}_{2} \mathrm{FC}(=\mathrm{O}) \mathrm{OH}$ & + & $\mathrm{CH}_{2} \mathrm{FCH}_{2} \bullet$ & -57.42 & -58.11 & -56.53 & -57.73 & -58.34 & -58.34 \\
\hline \multirow[t]{4}{*}{$\mathrm{CH}_{2} \cdot \mathrm{C}(=\mathrm{O}) \mathrm{OH}$} & + & $\mathrm{CH}_{3} \mathrm{CF}_{3}$ & $\rightarrow$ & $\mathrm{CH}_{2} \mathrm{FC}(=\mathrm{O}) \mathrm{OH}$ & + & $\mathrm{CH}_{3} \mathrm{CF}_{2} \bullet$ & -58.59 & -56.47 & -57.62 & -58.74 & -58.91 & -58.91 \\
\hline & & & & & & Average & -57.44 & -57.30 & -56.66 & -57.35 & -57.64 & -57.70 \\
\hline & & & & & & Uncertainty & 0.96 & 1.40 & 0.99 & 1.02 & 0.87 & 0.94 \\
\hline & & $\mathrm{CHF} \cdot \mathrm{C}(=\mathrm{O}) \mathrm{C}$ & & & & & & & & & & \\
\hline $\mathrm{CHF} \cdot \mathrm{C}(=\mathrm{O}) \mathrm{OH}$ & + & $\mathrm{CH}_{3} \mathrm{C}(=\mathrm{O}) \mathrm{OH}$ & $\rightarrow$ & $\mathrm{CH}_{2} \mathrm{FC}(=\mathrm{O}) \mathrm{OH}$ & + & $\mathrm{CH}_{2} \cdot \mathrm{C}(=\mathrm{O}) \mathrm{OH}$ & -101.93 & -102.49 & -103.60 & -101.84 & -102.41 & -101.70 \\
\hline $\mathrm{CHF} \cdot \mathrm{C}(=\mathrm{O}) \mathrm{OH}$ & + & $\mathrm{CH}_{3} \mathrm{OH}$ & $\rightarrow$ & $\mathrm{CH}_{2} \mathrm{FC}(=\mathrm{O}) \mathrm{OH}$ & + & $\mathrm{CH}_{2} \cdot \mathrm{OH}$ & -101.77 & -102.63 & -102.33 & -101.17 & -102.45 & -101.66 \\
\hline $\mathrm{CHF} \cdot \mathrm{C}(=\mathrm{O}) \mathrm{OH}$ & + & $\mathrm{CH}_{3} \mathrm{CH}_{2} \mathrm{~F}$ & $\rightarrow$ & $\mathrm{CH}_{2} \mathrm{FC}(=\mathrm{O}) \mathrm{OH}$ & + & $\mathrm{CH}_{2} \mathrm{FCH}_{2} \bullet$ & -101.28 & -102.24 & -103.24 & -101.45 & -102.63 & -100.92 \\
\hline $\mathrm{CHF} \cdot \mathrm{C}(=\mathrm{O}) \mathrm{OH}$ & + & $\mathrm{CH}_{3} \mathrm{CHF}_{2}$ & $\rightarrow$ & $\mathrm{CH}_{2} \mathrm{FC}(=\mathrm{O}) \mathrm{OH}$ & + & $\mathrm{CHF}_{2} \mathrm{CH}_{2} \bullet$ & -101.49 & -102.32 & -103.47 & -101.66 & -102.19 & -100.78 \\
\hline $\mathrm{CHF} \cdot \mathrm{C}(=\mathrm{O}) \mathrm{OH}$ & + & $\mathrm{CH}_{2} \mathrm{FCH}_{2} \mathrm{~F}$ & $\rightarrow$ & $\mathrm{CH}_{2} \mathrm{FC}(=\mathrm{O}) \mathrm{OH}$ & + & $\mathrm{CH}_{2} \mathrm{FCHF} \bullet$ & -100.89 & -101.34 & -100.58 & -101.11 & -101.45 & -100.60 \\
\hline \multirow[t]{3}{*}{$\mathrm{CHF} \cdot \mathrm{C}(=\mathrm{O}) \mathrm{OH}$} & + & $\mathrm{CH}_{3} \mathrm{CF}_{3}$ & $\rightarrow$ & $\mathrm{CH}_{2} \mathrm{FC}(=\mathrm{O}) \mathrm{OH}$ & + & $\mathrm{CF}_{3} \mathrm{CH}_{2} \bullet$ & -102.90 & -103.70 & -104.78 & -103.17 & -103.89 & -101.93 \\
\hline & & & & & & Average & -101.71 & -102.45 & -103.00 & -101.73 & -102.50 & -101.27 \\
\hline & & & & & & Uncertainty & 1.13 & 1.51 & 1.15 & 1.17 & 1.07 & 1.11 \\
\hline
\end{tabular}




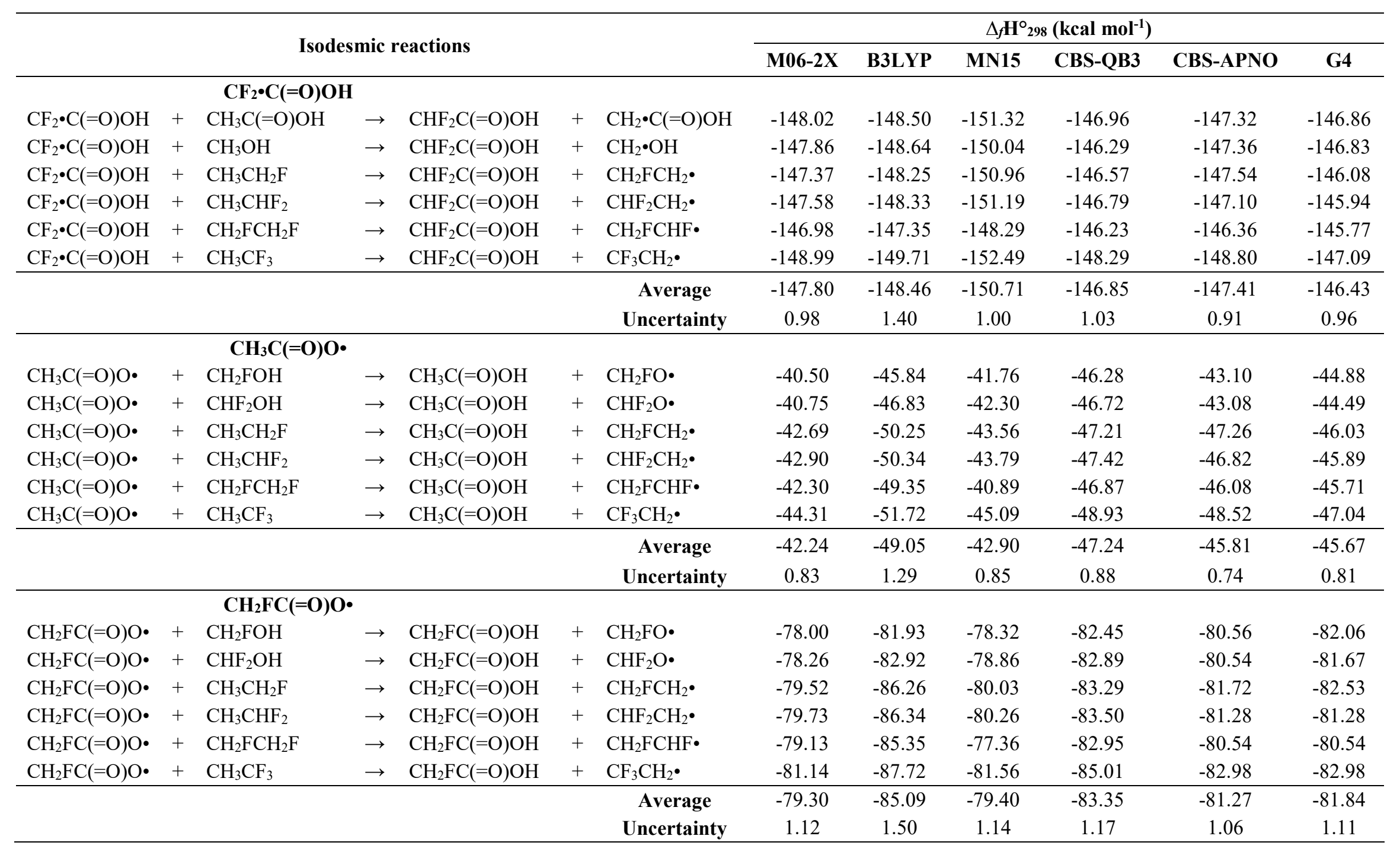




\begin{tabular}{|c|c|c|c|c|c|c|c|c|c|c|c|}
\hline \multirow{2}{*}{\multicolumn{6}{|c|}{ Isodesmic reactions }} & \multicolumn{6}{|c|}{$\Delta_{f} \mathbf{H}^{\circ}{ }_{298}\left(\mathrm{kcal} \mathrm{mol}^{-1}\right)$} \\
\hline & & & & & & \multirow[t]{2}{*}{ M06-2X } & \multirow[t]{2}{*}{ B3LYP } & \multirow[t]{2}{*}{ MN15 } & \multirow[t]{2}{*}{ CBS-QB3 } & \multirow[t]{2}{*}{ CBS-APNO } & \multirow[t]{2}{*}{ G4 } \\
\hline & & $\mathrm{CHF}_{2} \mathrm{C}(=$ & & & & & & & & & \\
\hline $\mathrm{CHF}_{2} \mathrm{C}(=\mathrm{O}) \mathrm{O} \bullet$ & + & $\mathrm{CHF}_{2} \mathrm{OH}$ & $\rightarrow$ & $\mathrm{CHF}_{2} \mathrm{C}(=\mathrm{O}) \mathrm{OH}$ & $\mathrm{CHF}_{2} \mathrm{O} \bullet$ & -125.35 & -129.95 & -125.79 & -130.12 & -127.29 & -129.18 \\
\hline $\mathrm{CHF}_{2} \mathrm{C}(=\mathrm{O}) \mathrm{O} \bullet$ & + & $\mathrm{CH}_{2} \mathrm{FOH}$ & $\rightarrow$ & $\mathrm{CHF}_{2} \mathrm{C}(=\mathrm{O}) \mathrm{OH}$ & $\mathrm{CH}_{2} \mathrm{FO} \bullet$ & -125.09 & -128.97 & -125.25 & -129.68 & -127.32 & -129.57 \\
\hline $\mathrm{CHF}_{2} \mathrm{C}(=\mathrm{O}) \mathrm{O} \bullet$ & + & $\mathrm{CH}_{3} \mathrm{CH}_{2} \mathrm{~F}$ & $\rightarrow$ & $\mathrm{CHF}_{2} \mathrm{C}(=\mathrm{O}) \mathrm{OH}$ & $\mathrm{CH}_{2} \mathrm{FCH}_{2} \bullet$ & -126.65 & -133.33 & -127.00 & -130.56 & -128.52 & -130.08 \\
\hline $\mathrm{CHF}_{2} \mathrm{C}(=\mathrm{O}) \mathrm{O} \bullet$ & + & $\mathrm{CH}_{3} \mathrm{CHF}_{2}$ & $\rightarrow$ & $\mathrm{CHF}_{2} \mathrm{C}(=\mathrm{O}) \mathrm{OH}$ & $\mathrm{CHF}_{2} \mathrm{CH}_{2} \bullet$ & -126.87 & -133.42 & -127.23 & -130.78 & -128.08 & -129.95 \\
\hline $\mathrm{CHF}_{2} \mathrm{C}(=\mathrm{O}) \mathrm{O} \bullet$ & + & $\mathrm{CH}_{2} \mathrm{FCH}_{2} \mathrm{~F}$ & $\rightarrow$ & $\mathrm{CHF}_{2} \mathrm{C}(=\mathrm{O}) \mathrm{OH}$ & $\mathrm{CH}_{2} \mathrm{FCHF} \bullet$ & -126.26 & -132.43 & -124.33 & -130.23 & -127.34 & -129.77 \\
\hline \multirow[t]{3}{*}{$\mathrm{CHF}_{2} \mathrm{C}(=\mathrm{O}) \mathrm{O} \bullet$} & + & $\mathrm{CH}_{3} \mathrm{CF}_{3}$ & $\rightarrow$ & $\mathrm{CHF}_{2} \mathrm{C}(=\mathrm{O}) \mathrm{OH}$ & $\mathrm{CF}_{3} \mathrm{CH}_{2} \bullet$ & -128.28 & -134.80 & -128.53 & -132.28 & -129.78 & -131.10 \\
\hline & & & & & Average & -126.42 & -132.15 & -126.35 & -130.61 & -128.05 & -129.94 \\
\hline & & & & & Uncertainty & 0.97 & 1.39 & 1.00 & 1.02 & 0.91 & 0.96 \\
\hline & & $\mathrm{CF}_{3} \mathrm{C}(=\mathrm{C}$ & & & & & & & & & \\
\hline $\mathrm{CF}_{3} \mathrm{C}(=\mathrm{O}) \mathrm{O} \bullet$ & + & $\mathrm{CHF}_{2} \mathrm{OH}$ & $\rightarrow$ & $\mathrm{CF}_{3} \mathrm{C}(=\mathrm{O}) \mathrm{OH}$ & $\mathrm{CHF}_{2} \mathrm{O} \bullet$ & -179.35 & -184.53 & -179.76 & -184.56 & -182.02 & -184.02 \\
\hline $\mathrm{CF}_{3} \mathrm{C}(=\mathrm{O}) \mathrm{O} \bullet$ & + & $\mathrm{CH}_{2} \mathrm{FOH}$ & $\rightarrow$ & $\mathrm{CF}_{3} \mathrm{C}(=\mathrm{O}) \mathrm{OH}$ & $\mathrm{CH}_{2} \mathrm{FO} \bullet$ & -179.10 & -183.55 & -179.22 & -184.13 & -182.04 & -184.40 \\
\hline $\mathrm{CF}_{3} \mathrm{C}(=\mathrm{O}) \mathrm{O} \bullet$ & + & $\mathrm{CH}_{3} \mathrm{CH}_{2} \mathrm{~F}$ & $\rightarrow$ & $\mathrm{CF}_{3} \mathrm{C}(=\mathrm{O}) \mathrm{OH}$ & $\mathrm{CH}_{2} \mathrm{FCH}_{2} \bullet$ & -180.66 & -187.91 & -180.98 & -185.00 & -183.25 & -184.92 \\
\hline $\mathrm{CF}_{3} \mathrm{C}(=\mathrm{O}) \mathrm{O} \bullet$ & + & $\mathrm{CH}_{3} \mathrm{CHF}_{2}$ & $\rightarrow$ & $\mathrm{CF}_{3} \mathrm{C}(=\mathrm{O}) \mathrm{OH}$ & $+\mathrm{CHF}_{2} \mathrm{CH}_{2} \bullet$ & -180.87 & -188.00 & -181.20 & -185.22 & -182.81 & -184.78 \\
\hline $\mathrm{CF}_{3} \mathrm{C}(=\mathrm{O}) \mathrm{O} \bullet$ & + & $\mathrm{CH}_{2} \mathrm{FCH}_{2} \mathrm{~F}$ & $\rightarrow$ & $\mathrm{CF}_{3} \mathrm{C}(=\mathrm{O}) \mathrm{OH}$ & $\mathrm{CH}_{2} \mathrm{FCHF} \bullet$ & -180.27 & -187.01 & -178.31 & -184.67 & -182.07 & -184.60 \\
\hline \multirow[t]{3}{*}{$\mathrm{CF}_{3} \mathrm{C}(=\mathrm{O}) \mathrm{O} \bullet$} & + & $\mathrm{CH}_{3} \mathrm{CF}_{3}$ & $\rightarrow$ & $\mathrm{CF}_{3} \mathrm{C}(=\mathrm{O}) \mathrm{OH}$ & $\mathrm{CF}_{3} \mathrm{CH}_{2} \bullet$ & -182.28 & -189.38 & -182.51 & -186.73 & -184.51 & -185.93 \\
\hline & & & & & Average & -180.42 & -186.73 & -180.33 & -185.05 & -182.78 & -184.78 \\
\hline & & & & & Uncertainty & 1.03 & 1.43 & 1.05 & 1.08 & 0.97 & 1.02 \\
\hline
\end{tabular}




\begin{tabular}{|c|c|c|c|c|c|c|c|c|c|c|c|}
\hline \multirow{2}{*}{\multicolumn{6}{|c|}{ Isodesmic reactions }} & \multicolumn{6}{|c|}{$\Delta_{f} \mathbf{H}^{\circ}{ }_{298}\left(\mathrm{kcal} \mathrm{mol}^{-1}\right)$} \\
\hline & & & & & & \multirow{2}{*}{ M06-2X } & \multirow[t]{2}{*}{ B3LYP } & \multirow[t]{2}{*}{ MN15 } & \multirow[t]{2}{*}{ CBS-QB3 } & \multirow[t]{2}{*}{ CBS-APNO } & \multirow[t]{2}{*}{ G4 } \\
\hline & & $\mathrm{CH}_{2} \cdot \mathrm{CH}_{2} \mathrm{C}(=\mathrm{O}$ & & & & & & & & & \\
\hline $\mathrm{CH}_{2} \cdot \mathrm{CH}_{2} \mathrm{C}(=\mathrm{O}) \mathrm{OH}$ & + & $\mathrm{CH}_{3} \mathrm{C}(=\mathrm{O}) \mathrm{OH}$ & $\rightarrow$ & $\mathrm{CH}_{3} \mathrm{CH}_{2} \mathrm{C}(=\mathrm{O}) \mathrm{OH}$ & $\mathrm{CH}_{2} \bullet \mathrm{C}(=\mathrm{O}) \mathrm{OH}$ & -59.11 & -58.75 & -59.33 & -59.14 & -59.14 & -59.79 \\
\hline $\mathrm{CH}_{2} \cdot \mathrm{CH}_{2} \mathrm{C}(=\mathrm{O}) \mathrm{OH}$ & + & $\mathrm{CH}_{3} \mathrm{CHF}_{2}$ & $\rightarrow$ & $\mathrm{CH}_{3} \mathrm{CH}_{2} \mathrm{C}(=\mathrm{O}) \mathrm{OH}$ & $\mathrm{CH}_{2} \mathrm{FCHF} \bullet$ & -58.22 & -56.45 & -57.59 & -58.34 & -58.52 & -58.61 \\
\hline $\mathrm{CH}_{2} \cdot \mathrm{CH}_{2} \mathrm{C}(=\mathrm{O}) \mathrm{OH}$ & + & $\mathrm{CH}_{3} \mathrm{CH}_{2} \mathrm{~F}$ & $\rightarrow$ & $\mathrm{CH}_{3} \mathrm{CH}_{2} \mathrm{C}(=\mathrm{O}) \mathrm{OH}$ & $\mathrm{CH}_{2} \mathrm{FCH}_{2} \bullet$ & -58.46 & -58.50 & -58.97 & -58.75 & -59.37 & -59.00 \\
\hline $\mathrm{CH}_{2} \cdot \mathrm{CH}_{2} \mathrm{C}(=\mathrm{O}) \mathrm{OH}$ & + & $\mathrm{CH}_{3} \mathrm{CH}_{2} \mathrm{~F}$ & $\rightarrow$ & $\mathrm{CH}_{3} \mathrm{CH}_{2} \mathrm{C}(=\mathrm{O}) \mathrm{OH}$ & $+\mathrm{CH}_{3} \mathrm{CHF} \bullet$ & -58.47 & -58.40 & -57.33 & -58.53 & -58.32 & -59.26 \\
\hline $\mathrm{CH}_{2} \cdot \mathrm{CH}_{2} \mathrm{C}(=\mathrm{O}) \mathrm{OH}$ & + & $\mathrm{CH}_{3} \mathrm{CHF}_{2}$ & $\rightarrow$ & $\mathrm{CH}_{3} \mathrm{CH}_{2} \mathrm{C}(=\mathrm{O}) \mathrm{OH}$ & $\mathrm{CHF}_{2} \mathrm{CH}_{2} \bullet$ & -58.67 & -58.58 & -59.20 & -58.97 & -58.92 & -58.86 \\
\hline $\mathrm{CH}_{2} \cdot \mathrm{CH}_{2} \mathrm{C}(=\mathrm{O}) \mathrm{OH}$ & + & $\mathrm{CH}_{2} \mathrm{FCH}_{2} \mathrm{~F}$ & $\rightarrow$ & $\mathrm{CH}_{3} \mathrm{CH}_{2} \mathrm{C}(=\mathrm{O}) \mathrm{OH}$ & $+\mathrm{CH}_{2} \mathrm{FCHF} \bullet$ & -58.07 & -57.60 & -56.30 & -58.41 & -58.18 & -58.69 \\
\hline $\mathrm{CH}_{2} \cdot \mathrm{CH}_{2} \mathrm{C}(=\mathrm{O}) \mathrm{OH}$ & + & $\mathrm{CH}_{3} \mathrm{CF}_{3}$ & $\rightarrow$ & $\mathrm{CH}_{3} \mathrm{CH}_{2} \mathrm{C}(=\mathrm{O}) \mathrm{OH}$ & $+\mathrm{CF}_{3} \mathrm{CH}_{2} \bullet$ & -60.08 & -59.96 & -60.51 & -60.47 & -60.62 & -60.02 \\
\hline \multirow[t]{4}{*}{$\mathrm{CH}_{2} \cdot \mathrm{CH}_{2} \mathrm{C}(=\mathrm{O}) \mathrm{OH}$} & + & $\mathrm{CH}_{3} \mathrm{CH}_{3}$ & $\rightarrow$ & $\mathrm{CH}_{3} \mathrm{CH}_{2} \mathrm{C}(=\mathrm{O}) \mathrm{OH}$ & $+\mathrm{CH}_{3} \mathrm{CH}_{2} \bullet$ & -59.07 & -59.02 & -59.09 & -58.87 & -58.95 & -59.71 \\
\hline & & & & & Average & -58.77 & -58.41 & -58.54 & -58.94 & -59.00 & -59.24 \\
\hline & & & & & Uncertainty & 0.84 & 1.30 & 0.86 & 0.90 & 0.75 & 0.82 \\
\hline & & $\mathrm{CH}_{2} \cdot \mathrm{CHFC}(=\mathrm{O}$ & & & & & & & & & \\
\hline $\mathrm{CH}_{2} \cdot \mathrm{CHFC}(=\mathrm{O}) \mathrm{OH}$ & + & $\mathrm{CH}_{3} \mathrm{CH}_{3}$ & $\rightarrow$ & $\mathrm{CH}_{3} \mathrm{CHFC}(=\mathrm{O}) \mathrm{OH}$ & $+\mathrm{CH}_{3} \mathrm{CH}_{2} \bullet$ & -100.49 & -100.68 & -100.56 & -100.58 & -100.61 & -101.77 \\
\hline $\mathrm{CH}_{2} \cdot \mathrm{CHFC}(=\mathrm{O}) \mathrm{OH}$ & + & $\mathrm{CH}_{3} \mathrm{CH}_{2} \mathrm{~F}$ & $\rightarrow$ & $\mathrm{CH}_{3} \mathrm{CHFC}(=\mathrm{O}) \mathrm{OH}$ & $+\mathrm{CH}_{3} \mathrm{CHF} \bullet$ & -99.89 & -100.05 & -98.80 & -100.23 & -99.98 & -101.31 \\
\hline $\mathrm{CH}_{2} \cdot \mathrm{CHFC}(=\mathrm{O}) \mathrm{OH}$ & + & $\mathrm{CH}_{3} \mathrm{CHF}_{2}$ & $\rightarrow$ & $\mathrm{CH}_{3} \mathrm{CHFC}(=\mathrm{O}) \mathrm{OH}$ & $+\mathrm{CH}_{3} \mathrm{CF}_{2} \bullet$ & -99.80 & -99.75 & -98.54 & -100.32 & -99.66 & -100.97 \\
\hline $\mathrm{CH}_{2} \cdot \mathrm{CHFC}(=\mathrm{O}) \mathrm{OH}$ & + & $\mathrm{CH}_{3} \mathrm{CH}_{2} \mathrm{~F}$ & $\rightarrow$ & $\mathrm{CH}_{3} \mathrm{CHFC}(=\mathrm{O}) \mathrm{OH}$ & $+\mathrm{CH}_{2} \mathrm{FCH}_{2} \bullet$ & -99.88 & -100.16 & -100.44 & -100.46 & -101.03 & -101.05 \\
\hline $\mathrm{CH}_{2} \cdot \mathrm{CHFC}(=\mathrm{O}) \mathrm{OH}$ & + & $\mathrm{CH}_{3} \mathrm{CHF}_{2}$ & $\rightarrow$ & $\mathrm{CH}_{3} \mathrm{CHFC}(=\mathrm{O}) \mathrm{OH}$ & $+\mathrm{CHF}_{2} \mathrm{CH}_{2} \bullet$ & -100.09 & -100.24 & -100.67 & -100.67 & -100.58 & -100.92 \\
\hline $\mathrm{CH}_{2} \cdot \mathrm{CHFC}(=\mathrm{O}) \mathrm{OH}$ & + & $\mathrm{CH}_{2} \mathrm{FCH}_{2} \mathrm{~F}$ & $\rightarrow$ & $\mathrm{CH}_{3} \mathrm{CHFC}(=\mathrm{O}) \mathrm{OH}$ & $+\mathrm{CH}_{2} \mathrm{FCHF} \bullet$ & -99.49 & -99.25 & -97.77 & -100.12 & -99.84 & -100.74 \\
\hline \multirow[t]{3}{*}{$\mathrm{CH}_{2} \cdot \mathrm{CHFC}(=\mathrm{O}) \mathrm{OH}$} & + & $\mathrm{CH}_{3} \mathrm{CF}_{3}$ & $\rightarrow$ & $\mathrm{CH}_{3} \mathrm{CHFC}(=\mathrm{O}) \mathrm{OH}$ & $+\mathrm{CF}_{3} \mathrm{CH}_{2} \bullet$ & -101.50 & -101.62 & -101.97 & -102.18 & -102.28 & -102.07 \\
\hline & & & & & Average & -100.16 & -100.25 & -99.82 & -100.65 & -100.57 & -101.26 \\
\hline & & & & & Uncertainty & 1.12 & 1.50 & 1.14 & 1.17 & 1.06 & 1.11 \\
\hline
\end{tabular}




\begin{tabular}{|c|c|c|c|c|c|c|c|c|c|c|c|}
\hline \multirow{2}{*}{\multicolumn{6}{|c|}{ Isodesmic reactions }} & \multicolumn{6}{|c|}{$\Delta_{f} \mathrm{H}^{\circ}{ }_{298}\left(\mathrm{kcal} \mathrm{mol}^{-1}\right)$} \\
\hline & & & & & & \multirow[t]{2}{*}{ M06-2X } & \multirow[t]{2}{*}{ B3LYP } & \multirow[t]{2}{*}{ MN15 } & \multirow[t]{2}{*}{ CBS-QB3 } & \multirow[t]{2}{*}{ CBS-APNO } & \multirow[t]{2}{*}{ G4 } \\
\hline & & $\mathrm{CH}_{2} \cdot \mathrm{CF}_{2} \mathrm{C}($ & & & & & & & & & \\
\hline $\mathrm{CH}_{2} \cdot \mathrm{CF}_{2} \mathrm{C}(=\mathrm{O}) \mathrm{OH}$ & + & $\mathrm{CH}_{3} \mathrm{CHF}_{2}$ & $\rightarrow$ & $\mathrm{CH}_{3} \mathrm{CF}_{2} \mathrm{C}(=\mathrm{O}) \mathrm{OH}$ & $+\mathrm{CH}_{3} \mathrm{CF}_{2} \bullet$ & -150.84 & -150.71 & -149.05 & -150.41 & -149.88 & -151.57 \\
\hline $\mathrm{CH}_{2} \cdot \mathrm{CF}_{2} \mathrm{C}(=\mathrm{O}) \mathrm{OH}$ & + & $\mathrm{CH}_{3} \mathrm{CH}_{3}$ & $\rightarrow$ & $\mathrm{CH}_{3} \mathrm{CF}_{2} \mathrm{C}(=\mathrm{O}) \mathrm{OH}$ & $+\mathrm{CH}_{3} \mathrm{CH}_{2} \bullet$ & -151.53 & -151.64 & -151.06 & -150.67 & -150.83 & -152.37 \\
\hline $\mathrm{CH}_{2} \cdot \mathrm{CF}_{2} \mathrm{C}(=\mathrm{O}) \mathrm{OH}$ & + & $\mathrm{CH}_{3} \mathrm{CH}_{2} \mathrm{~F}$ & $\rightarrow$ & $\mathrm{CH}_{3} \mathrm{CF}_{2} \mathrm{C}(=\mathrm{O}) \mathrm{OH}$ & $+\mathrm{CH}_{3} \mathrm{CHF} \bullet$ & -150.93 & -151.01 & -149.30 & -150.33 & -150.19 & -151.91 \\
\hline $\mathrm{CH}_{2} \cdot \mathrm{CF}_{2} \mathrm{C}(=\mathrm{O}) \mathrm{OH}$ & + & $\mathrm{CH}_{3} \mathrm{CH}_{2} \mathrm{~F}$ & $\rightarrow$ & $\mathrm{CH}_{3} \mathrm{CF}_{2} \mathrm{C}(=\mathrm{O}) \mathrm{OH}$ & $+\mathrm{CH}_{2} \mathrm{FCH}_{2} \cdot$ & -150.92 & -151.12 & -150.94 & -150.55 & -151.24 & -151.66 \\
\hline $\mathrm{CH}_{2} \cdot \mathrm{CF}_{2} \mathrm{C}(=\mathrm{O}) \mathrm{OH}$ & + & $\mathrm{CH}_{3} \mathrm{CHF}_{2}$ & $\rightarrow$ & $\mathrm{CH}_{3} \mathrm{CF}_{2} \mathrm{C}(=\mathrm{O}) \mathrm{OH}$ & $+\mathrm{CHF}_{2} \mathrm{CH}_{2} \bullet$ & -151.13 & -151.20 & -151.17 & -150.77 & -150.80 & -151.52 \\
\hline $\mathrm{CH}_{2} \cdot \mathrm{CF}_{2} \mathrm{C}(=\mathrm{O}) \mathrm{OH}$ & + & $\mathrm{CH}_{2} \mathrm{FCH}_{2} \mathrm{~F}$ & $\rightarrow$ & $\mathrm{CH}_{3} \mathrm{CF}_{2} \mathrm{C}(=\mathrm{O}) \mathrm{OH}$ & $+\mathrm{CH}_{2} \mathrm{FCHF} \bullet$ & -150.53 & -150.21 & -148.27 & -150.21 & -150.06 & -151.34 \\
\hline \multirow[t]{3}{*}{$\mathrm{CH}_{2} \cdot \mathrm{CF}_{2} \mathrm{C}(=\mathrm{O}) \mathrm{OH}$} & + & $\mathrm{CH}_{3} \mathrm{CF}_{3}$ & $\rightarrow$ & $\mathrm{CH}_{3} \mathrm{CF}_{2} \mathrm{C}(=\mathrm{O}) \mathrm{OH}$ & $+\mathrm{CF}_{3} \mathrm{CH}_{2} \bullet$ & -152.54 & -152.58 & -152.48 & -152.27 & -152.50 & -152.67 \\
\hline & & & & & Average & -151.20 & -151.21 & -150.33 & -150.74 & -150.79 & -151.86 \\
\hline & & & & & Uncertainty & 0.98 & 1.40 & 1.01 & 1.03 & 0.92 & 0.97 \\
\hline & & $\mathrm{CHF} \cdot \mathrm{CH}_{2} \mathrm{C}($ & & & & & & & & & \\
\hline $\mathrm{CHF} \cdot \mathrm{CH}_{2} \mathrm{C}(=\mathrm{O}) \mathrm{OH}$ & + & $\mathrm{CH}_{3} \mathrm{CH}_{3}$ & $\rightarrow$ & $\mathrm{CH}_{2} \mathrm{FCH}_{2} \mathrm{C}(=\mathrm{O}) \mathrm{OH}$ & $+\mathrm{CH}_{3} \mathrm{CH}_{2} \bullet$ & -104.10 & -104.19 & -105.13 & -103.67 & -103.90 & -104.58 \\
\hline $\mathrm{CHF} \cdot \mathrm{CH}_{2} \mathrm{C}(=\mathrm{O}) \mathrm{OH}$ & + & $\mathrm{CH}_{3} \mathrm{CH}_{2} \mathrm{~F}$ & $\rightarrow$ & $\mathrm{CH}_{2} \mathrm{FCH}_{2} \mathrm{C}(=\mathrm{O}) \mathrm{OH}$ & $+\mathrm{CH}_{3} \mathrm{CHF} \bullet$ & -103.50 & -103.56 & -103.37 & -103.33 & -103.26 & -104.12 \\
\hline $\mathrm{CHF} \cdot \mathrm{CH}_{2} \mathrm{C}(=\mathrm{O}) \mathrm{OH}$ & + & $\mathrm{CH}_{3} \mathrm{CHF}_{2}$ & $\rightarrow$ & $\mathrm{CH}_{2} \mathrm{FCH}_{2} \mathrm{C}(=\mathrm{O}) \mathrm{OH}$ & $+\mathrm{CH}_{3} \mathrm{CF}_{2} \bullet$ & -103.41 & -103.26 & -103.11 & -103.41 & -102.95 & -103.78 \\
\hline $\mathrm{CHF} \cdot \mathrm{CH}_{2} \mathrm{C}(=\mathrm{O}) \mathrm{OH}$ & + & $\mathrm{CH}_{3} \mathrm{CH}_{2} \mathrm{~F}$ & $\rightarrow$ & $\mathrm{CH}_{2} \mathrm{FCH}_{2} \mathrm{C}(=\mathrm{O}) \mathrm{OH}$ & $+\mathrm{CH}_{2} \mathrm{FCH}_{2} \bullet$ & -103.87 & -104.05 & -105.39 & -103.93 & -104.70 & -104.25 \\
\hline $\mathrm{CHF} \cdot \mathrm{CH}_{2} \mathrm{C}(=\mathrm{O}) \mathrm{OH}$ & + & $\mathrm{CH}_{3} \mathrm{CHF}_{2}$ & $\rightarrow$ & $\mathrm{CH}_{2} \mathrm{FCH}_{2} \mathrm{C}(=\mathrm{O}) \mathrm{OH}$ & $+\mathrm{CHF}_{2} \mathrm{CH}_{2} \bullet$ & -104.09 & -104.13 & -105.62 & -104.15 & -104.25 & -104.11 \\
\hline $\mathrm{CHF} \cdot \mathrm{CH}_{2} \mathrm{C}(=\mathrm{O}) \mathrm{OH}$ & + & $\mathrm{CH}_{2} \mathrm{FCH}_{2} \mathrm{~F}$ & $\rightarrow$ & $\mathrm{CH}_{2} \mathrm{FCH}_{2} \mathrm{C}(=\mathrm{O}) \mathrm{OH}$ & $+\mathrm{CH}_{2} \mathrm{FCHF} \bullet$ & -103.48 & -103.14 & -102.72 & -103.60 & -103.51 & -103.93 \\
\hline \multirow[t]{3}{*}{$\mathrm{CHF} \cdot \mathrm{CH}_{2} \mathrm{C}(=\mathrm{O}) \mathrm{OH}$} & + & $\mathrm{CH}_{3} \mathrm{CF}_{3}$ & $\rightarrow$ & $\mathrm{CH}_{2} \mathrm{FCH}_{2} \mathrm{C}(=\mathrm{O}) \mathrm{OH}$ & $+\mathrm{CF}_{3} \mathrm{CH}_{2} \bullet$ & -105.50 & -105.51 & -106.93 & -105.66 & -105.95 & -105.26 \\
\hline & & & & & Average & -103.99 & -103.98 & -104.61 & -103.96 & -104.07 & -104.29 \\
\hline & & & & & Uncertainty & 1.12 & 1.50 & 1.14 & 1.16 & 1.06 & 1.11 \\
\hline
\end{tabular}




\begin{tabular}{|c|c|c|c|c|c|c|c|c|c|c|c|}
\hline \multirow{2}{*}{\multicolumn{6}{|c|}{ Isodesmic reactions }} & \multicolumn{6}{|c|}{$\Delta_{f} \mathbf{H}^{\circ}{ }_{298}\left(\mathrm{kcal} \mathrm{mol}^{-1}\right)$} \\
\hline & & & & & & \multirow[t]{2}{*}{ M06-2X } & \multirow[t]{2}{*}{ B3LYP } & \multirow[t]{2}{*}{ MN15 } & \multirow[t]{2}{*}{ CBS-QB3 } & \multirow[t]{2}{*}{ CBS-APNO } & \multirow[t]{2}{*}{ G4 } \\
\hline & & $\mathrm{CHF} \cdot \mathrm{CHFC}($ & & & & & & & & & \\
\hline $\mathrm{CHF} \cdot \mathrm{CHFC}(=\mathrm{O}) \mathrm{OH}$ & + & $\mathrm{CH}_{3} \mathrm{CHF}_{2}$ & $\rightarrow$ & $\mathrm{CH}_{2} \mathrm{FCHFC}(=\mathrm{O}) \mathrm{OH}$ & $\mathrm{CH}_{3} \mathrm{CF}_{2} \bullet$ & -143.92 & -144.54 & -143.76 & -143.43 & -143.39 & -144.10 \\
\hline $\mathrm{CHF} \cdot \mathrm{CHFC}(=\mathrm{O}) \mathrm{OH}$ & + & $\mathrm{CH}_{3} \mathrm{CH}_{3}$ & $\rightarrow$ & $\mathrm{CH}_{2} \mathrm{FCHFC}(=\mathrm{O}) \mathrm{OH}$ & $+\mathrm{CH}_{3} \mathrm{CH}_{2} \bullet$ & -144.60 & -145.47 & -145.77 & -143.69 & -144.34 & -144.90 \\
\hline $\mathrm{CHF} \cdot \mathrm{CHFC}(=\mathrm{O}) \mathrm{OH}$ & + & $\mathrm{CH}_{3} \mathrm{CH}_{2} \mathrm{~F}$ & $\rightarrow$ & $\mathrm{CH}_{2} \mathrm{FCHFC}(=\mathrm{O}) \mathrm{OH}$ & $+\mathrm{CH}_{3} \mathrm{CHF} \bullet$ & -144.00 & -144.84 & -144.01 & -143.34 & -143.70 & -144.44 \\
\hline $\mathrm{CHF} \cdot \mathrm{CHFC}(=\mathrm{O}) \mathrm{OH}$ & + & $\mathrm{CH}_{3} \mathrm{CH}_{2} \mathrm{~F}$ & $\rightarrow$ & $\mathrm{CH}_{2} \mathrm{FCHFC}(=\mathrm{O}) \mathrm{OH}$ & $+\mathrm{CH}_{2} \mathrm{FCH}_{2} \bullet$ & -143.99 & -144.94 & -145.65 & -143.57 & -144.75 & -144.18 \\
\hline $\mathrm{CHF} \cdot \mathrm{CHFC}(=\mathrm{O}) \mathrm{OH}$ & + & $\mathrm{CH}_{3} \mathrm{CHF}_{2}$ & $\rightarrow$ & $\mathrm{CH}_{2} \mathrm{FCHFC}(=\mathrm{O}) \mathrm{OH}$ & $+\mathrm{CHF}_{2} \mathrm{CH}_{2} \cdot$ & -144.21 & -145.02 & -145.88 & -143.78 & -144.31 & -144.04 \\
\hline $\mathrm{CHF} \cdot \mathrm{CHFC}(=\mathrm{O}) \mathrm{OH}$ & + & $\mathrm{CH}_{2} \mathrm{FCH}_{2} \mathrm{~F}$ & $\rightarrow$ & $\mathrm{CH}_{2} \mathrm{FCHFC}(=\mathrm{O}) \mathrm{OH}$ & $+\mathrm{CH}_{2} \mathrm{FCHF} \bullet$ & -143.60 & -144.04 & -142.99 & -143.23 & -143.57 & -143.87 \\
\hline \multirow[t]{3}{*}{$\mathrm{CHF} \cdot \mathrm{CHFC}(=\mathrm{O}) \mathrm{OH}$} & + & $\mathrm{CH}_{3} \mathrm{CF}_{3}$ & $\rightarrow$ & $\mathrm{CH}_{2} \mathrm{FCHFC}(=\mathrm{O}) \mathrm{OH}$ & $+\mathrm{CF}_{3} \mathrm{CH}_{2} \bullet$ & -145.62 & -146.40 & -147.19 & -145.29 & -146.01 & -145.20 \\
\hline & & & & & Average & -144.28 & -145.04 & -145.04 & -143.76 & -144.30 & -144.39 \\
\hline & & & & & Uncertainty & 1.00 & 1.41 & 1.03 & 1.05 & 0.94 & 0.99 \\
\hline & & $\mathrm{CHF} \cdot \mathrm{CF}_{2} \mathrm{C}(=$ & & & & & & & & & \\
\hline $\mathrm{CHF} \cdot \mathrm{CF}_{2} \mathrm{C}(=\mathrm{O}) \mathrm{OH}$ & + & $\mathrm{CH}_{3} \mathrm{CH}_{3}$ & $\rightarrow$ & $\mathrm{CH}_{2} \mathrm{FCF}_{2} \mathrm{C}(=\mathrm{O}) \mathrm{OH}$ & $+\mathrm{CH}_{3} \mathrm{CH}_{2} \bullet$ & -192.75 & -193.40 & -193.37 & -192.02 & -191.72 & -193.77 \\
\hline $\mathrm{CHF} \cdot \mathrm{CF}_{2} \mathrm{C}(=\mathrm{O}) \mathrm{OH}$ & + & $\mathrm{CH}_{3} \mathrm{CH}_{2} \mathrm{~F}$ & $\rightarrow$ & $\mathrm{CH}_{2} \mathrm{FCF}_{2} \mathrm{C}(=\mathrm{O}) \mathrm{OH}$ & $+\mathrm{CH}_{3} \mathrm{CHF} \bullet$ & -192.15 & -192.78 & -191.61 & -191.68 & -191.08 & -193.31 \\
\hline $\mathrm{CHF} \cdot \mathrm{CF}_{2} \mathrm{C}(=\mathrm{O}) \mathrm{OH}$ & + & $\mathrm{CH}_{3} \mathrm{CHF}_{2}$ & $\rightarrow$ & $\mathrm{CH}_{2} \mathrm{FCF}_{2} \mathrm{C}(=\mathrm{O}) \mathrm{OH}$ & $+\mathrm{CH}_{3} \mathrm{CF}_{2} \bullet$ & -192.06 & -192.48 & -191.35 & -191.76 & -190.77 & -192.97 \\
\hline $\mathrm{CHF} \cdot \mathrm{CF}_{2} \mathrm{C}(=\mathrm{O}) \mathrm{OH}$ & + & $\mathrm{CH}_{3} \mathrm{CH}_{2} \mathrm{~F}$ & $\rightarrow$ & $\mathrm{CH}_{2} \mathrm{FCF}_{2} \mathrm{C}(=\mathrm{O}) \mathrm{OH}$ & $+\mathrm{CH}_{2} \mathrm{FCH}_{2} \cdot$ & -192.14 & -192.88 & -193.25 & -191.90 & -192.13 & -193.06 \\
\hline $\mathrm{CHF} \cdot \mathrm{CF}_{2} \mathrm{C}(=\mathrm{O}) \mathrm{OH}$ & + & $\mathrm{CH}_{3} \mathrm{CHF}_{2}$ & $\rightarrow$ & $\mathrm{CH}_{2} \mathrm{FCF}_{2} \mathrm{C}(=\mathrm{O}) \mathrm{OH}$ & $+\mathrm{CHF}_{2} \mathrm{CH}_{2} \cdot$ & -192.35 & -192.96 & -193.48 & -192.12 & -191.69 & -192.92 \\
\hline $\mathrm{CHF} \cdot \mathrm{CF}_{2} \mathrm{C}(=\mathrm{O}) \mathrm{OH}$ & + & $\mathrm{CH}_{2} \mathrm{FCH}_{2} \mathrm{~F}$ & $\rightarrow$ & $\mathrm{CH}_{2} \mathrm{FCF}_{2} \mathrm{C}(=\mathrm{O}) \mathrm{OH}$ & $+\mathrm{CH}_{2} \mathrm{FCHF} \bullet$ & -191.75 & -191.98 & -190.58 & -191.56 & -190.95 & -192.74 \\
\hline \multirow[t]{3}{*}{$\mathrm{CHF} \cdot \mathrm{CF}_{2} \mathrm{C}(=\mathrm{O}) \mathrm{OH}$} & + & $\mathrm{CH}_{3} \mathrm{CF}_{3}$ & $\rightarrow$ & $\mathrm{CH}_{2} \mathrm{FCF}_{2} \mathrm{C}(=\mathrm{O}) \mathrm{OH}$ & $+\mathrm{CF}_{3} \mathrm{CH}_{2} \bullet$ & -193.76 & -194.34 & -194.78 & -193.62 & -193.39 & -194.07 \\
\hline & & & & & Average & -192.42 & -192.97 & -192.63 & -192.09 & -191.68 & -193.26 \\
\hline & & & & & Uncertainty & 1.06 & 1.45 & 1.08 & 1.11 & 1.00 & 1.05 \\
\hline
\end{tabular}




\begin{tabular}{|c|c|c|c|c|c|c|c|c|c|c|c|}
\hline \multirow{2}{*}{\multicolumn{6}{|c|}{ Isodesmic reactions }} & \multicolumn{6}{|c|}{$\Delta_{f} \mathbf{H}^{\circ}{ }_{298}\left(\mathrm{kcal} \mathrm{mol}^{-1}\right)$} \\
\hline & & & & & & M06-2X & B3LYP & MN15 & CBS-QB3 & CBS-APNO & G4 \\
\hline \multicolumn{12}{|c|}{$\mathrm{CF}_{2} \cdot \mathrm{CH}_{2} \mathrm{C}(=\mathrm{O}) \mathrm{OH}$} \\
\hline $\mathrm{CF}_{2} \cdot \mathrm{CH}_{2} \mathrm{C}(=\mathrm{O}) \mathrm{OH}$ & + & $\mathrm{CH}_{3} \mathrm{CH}_{2} \mathrm{~F}$ & $\rightarrow$ & $\mathrm{CHF}_{2} \mathrm{CH}_{2} \mathrm{C}(=\mathrm{O}) \mathrm{OH}$ & $+\mathrm{CH}_{3} \mathrm{CHF} \bullet$ & -154.94 & -155.10 & -154.99 & -154.61 & -154.98 & -155.92 \\
\hline $\mathrm{CF}_{2} \cdot \mathrm{CH}_{2} \mathrm{C}(=\mathrm{O}) \mathrm{OH}$ & + & $\mathrm{CH}_{3} \mathrm{CH}_{3}$ & $\rightarrow$ & $\mathrm{CHF}_{2} \mathrm{CH}_{2} \mathrm{C}(=\mathrm{O}) \mathrm{OH}$ & $+\mathrm{CH}_{3} \mathrm{CH}_{2} \bullet$ & -155.54 & -155.73 & -156.75 & -154.96 & -155.62 & -156.38 \\
\hline $\mathrm{CF}_{2} \cdot \mathrm{CH}_{2} \mathrm{C}(=\mathrm{O}) \mathrm{OH}$ & + & $\mathrm{CH}_{3} \mathrm{CHF}_{2}$ & $\rightarrow$ & $\mathrm{CHF}_{2} \mathrm{CH}_{2} \mathrm{C}(=\mathrm{O}) \mathrm{OH}$ & $+\mathrm{CH}_{3} \mathrm{CF}_{2} \bullet$ & -154.86 & -154.80 & -154.74 & -154.70 & -154.67 & -155.58 \\
\hline $\mathrm{CF}_{2} \cdot \mathrm{CH}_{2} \mathrm{C}(=\mathrm{O}) \mathrm{OH}$ & + & $\mathrm{CH}_{3} \mathrm{CH}_{2} \mathrm{~F}$ & $\rightarrow$ & $\mathrm{CHF}_{2} \mathrm{CH}_{2} \mathrm{C}(=\mathrm{O}) \mathrm{OH}$ & $+\mathrm{CH}_{2} \mathrm{FCH}_{2} \bullet$ & -154.93 & -155.21 & -156.63 & -154.83 & -156.03 & -155.67 \\
\hline $\mathrm{CF}_{2} \cdot \mathrm{CH}_{2} \mathrm{C}(=\mathrm{O}) \mathrm{OH}$ & + & $\mathrm{CH}_{3} \mathrm{CHF}_{2}$ & $\rightarrow$ & $\mathrm{CHF}_{2} \mathrm{CH}_{2} \mathrm{C}(=\mathrm{O}) \mathrm{OH}$ & $+\mathrm{CHF}_{2} \mathrm{CH}_{2} \bullet$ & -155.14 & -155.29 & -156.86 & -155.05 & -155.59 & -155.53 \\
\hline $\mathrm{CF}_{2} \cdot \mathrm{CH}_{2} \mathrm{C}(=\mathrm{O}) \mathrm{OH}$ & + & $\mathrm{CH}_{2} \mathrm{FCH}_{2} \mathrm{~F}$ & $\rightarrow$ & $\mathrm{CHF}_{2} \mathrm{CH}_{2} \mathrm{C}(=\mathrm{O}) \mathrm{OH}$ & $+\mathrm{CH}_{2} \mathrm{FCHF} \bullet$ & -154.54 & -154.30 & -153.96 & -154.50 & -154.85 & -155.36 \\
\hline \multirow[t]{3}{*}{$\mathrm{CF}_{2} \cdot \mathrm{CH}_{2} \mathrm{C}(=\mathrm{O}) \mathrm{OH}$} & + & $\mathrm{CH}_{3} \mathrm{CF}_{3}$ & $\rightarrow$ & $\mathrm{CHF}_{2} \mathrm{CH}_{2} \mathrm{C}(=\mathrm{O}) \mathrm{OH}$ & $+\mathrm{CF}_{3} \mathrm{CH}_{2} \bullet$ & -156.55 & -156.67 & -158.17 & -156.56 & -157.29 & -156.68 \\
\hline & & & & & Average & -155.22 & -155.30 & -156.02 & -155.03 & -155.57 & -155.88 \\
\hline & & & & & Uncertainty & 1.00 & 1.41 & 1.03 & 1.05 & 0.94 & 0.99 \\
\hline & & $\mathrm{CF}_{2} \cdot \mathrm{CHFC}($ & & & & & & & & & \\
\hline $\mathrm{CF}_{2} \cdot \mathrm{CHFC}(=\mathrm{O}) \mathrm{OH}$ & + & $\mathrm{CH}_{3} \mathrm{CH}_{3}$ & $\rightarrow$ & $\mathrm{CHF}_{2} \mathrm{CHFC}(=\mathrm{O}) \mathrm{OH}$ & $+\mathrm{CH}_{3} \mathrm{CH}_{2} \bullet$ & -192.73 & -193.58 & -194.67 & -192.05 & -192.91 & -193.87 \\
\hline $\mathrm{CF}_{2} \cdot \mathrm{CHFC}(=\mathrm{O}) \mathrm{OH}$ & + & $\mathrm{CH}_{3} \mathrm{CH}_{2} \mathrm{~F}$ & $\rightarrow$ & $\mathrm{CHF}_{2} \mathrm{CHFC}(=\mathrm{O}) \mathrm{OH}$ & $+\mathrm{CH}_{3} \mathrm{CHF} \bullet$ & -192.13 & -192.96 & -192.91 & -191.70 & -192.28 & -193.41 \\
\hline $\mathrm{CF}_{2} \cdot \mathrm{CHFC}(=\mathrm{O}) \mathrm{OH}$ & + & $\mathrm{CH}_{3} \mathrm{CHF}_{2}$ & $\rightarrow$ & $\mathrm{CHF}_{2} \mathrm{CHFC}(=\mathrm{O}) \mathrm{OH}$ & $+\mathrm{CH}_{3} \mathrm{CF}_{2} \bullet$ & -192.05 & -192.66 & -192.65 & -191.79 & -191.96 & -193.07 \\
\hline $\mathrm{CF}_{2} \cdot \mathrm{CHFC}(=\mathrm{O}) \mathrm{OH}$ & + & $\mathrm{CH}_{3} \mathrm{CH}_{2} \mathrm{~F}$ & $\rightarrow$ & $\mathrm{CHF}_{2} \mathrm{CHFC}(=\mathrm{O}) \mathrm{OH}$ & $+\mathrm{CH}_{2} \mathrm{FCH}_{2} \bullet$ & -192.12 & -193.06 & -194.55 & -191.92 & -193.33 & -193.16 \\
\hline $\mathrm{CF}_{2} \cdot \mathrm{CHFC}(=\mathrm{O}) \mathrm{OH}$ & + & $\mathrm{CH}_{3} \mathrm{CHF}_{2}$ & $\rightarrow$ & $\mathrm{CHF}_{2} \mathrm{CHFC}(=\mathrm{O}) \mathrm{OH}$ & $+\mathrm{CHF}_{2} \mathrm{CH}_{2} \bullet$ & -192.34 & -193.14 & -194.78 & -192.14 & -192.88 & -193.02 \\
\hline $\mathrm{CF}_{2} \cdot \mathrm{CHFC}(=\mathrm{O}) \mathrm{OH}$ & + & $\mathrm{CH}_{2} \mathrm{FCH}_{2} \mathrm{~F}$ & $\rightarrow$ & $\mathrm{CHF}_{2} \mathrm{CHFC}(=\mathrm{O}) \mathrm{OH}$ & $+\mathrm{CH}_{2} \mathrm{FCHF} \bullet$ & -191.73 & -192.16 & -191.88 & -191.59 & -192.14 & -192.84 \\
\hline \multirow[t]{3}{*}{$\mathrm{CF}_{2} \cdot \mathrm{CHFC}(=\mathrm{O}) \mathrm{OH}$} & + & $\mathrm{CH}_{3} \mathrm{CF}_{3}$ & $\rightarrow$ & $\mathrm{CHF}_{2} \mathrm{CHFC}(=\mathrm{O}) \mathrm{OH}$ & $+\mathrm{CF}_{3} \mathrm{CH}_{2} \bullet$ & -193.75 & -194.52 & -196.08 & -193.65 & -194.58 & -194.17 \\
\hline & & & & & Average & -192.41 & -193.15 & -193.93 & -192.12 & -192.87 & -193.36 \\
\hline & & & & & Uncertainty & 1.07 & 1.46 & 1.09 & 1.12 & 1.01 & 1.06 \\
\hline
\end{tabular}




\begin{tabular}{|c|c|c|c|c|c|c|c|c|c|c|c|c|}
\hline \multirow{2}{*}{\multicolumn{7}{|c|}{ Isodesmic reactions }} & \multicolumn{6}{|c|}{$\Delta_{f} \mathrm{H}^{\circ} 298\left(\mathrm{kcal} \mathrm{mol}^{-1}\right)$} \\
\hline & & & & & & & \multirow[t]{2}{*}{ M06-2X } & \multirow[t]{2}{*}{ B3LYP } & \multirow[t]{2}{*}{ MN15 } & \multirow[t]{2}{*}{ CBS-QB3 } & \multirow[t]{2}{*}{ CBS-APNO } & \multirow[t]{2}{*}{ G4 } \\
\hline & & $\mathrm{CF}_{2} \cdot \mathrm{CF}_{2} \mathrm{C}(=$ & & & & & & & & & & \\
\hline $\mathrm{CF}_{2} \cdot \mathrm{CHFC}(=\mathrm{O}) \mathrm{OH}$ & + & $\mathrm{CH}_{3} \mathrm{CH}_{3}$ & $\rightarrow$ & $\mathrm{CHF}_{2} \mathrm{CF}_{2} \mathrm{C}(=\mathrm{O}) \mathrm{OH}$ & + & $\mathrm{CH}_{3} \mathrm{CH}_{2} \bullet$ & -240.56 & -241.23 & -242.14 & -240.24 & -240.93 & -242.27 \\
\hline $\mathrm{CF}_{2} \cdot \mathrm{CHFC}(=\mathrm{O}) \mathrm{OH}$ & + & $\mathrm{CH}_{3} \mathrm{CH}_{2} \mathrm{~F}$ & $\rightarrow$ & $\mathrm{CHF}_{2} \mathrm{CF}_{2} \mathrm{C}(=\mathrm{O}) \mathrm{OH}$ & + & $\mathrm{CH}_{3} \mathrm{CHF} \bullet$ & -239.96 & -240.60 & -240.38 & -239.90 & -240.29 & -241.81 \\
\hline $\mathrm{CF}_{2} \cdot \mathrm{CHFC}(=\mathrm{O}) \mathrm{OH}$ & + & $\mathrm{CH}_{3} \mathrm{CHF}_{2}$ & $\rightarrow$ & $\mathrm{CHF}_{2} \mathrm{CF}_{2} \mathrm{C}(=\mathrm{O}) \mathrm{OH}$ & + & $\mathrm{CH}_{3} \mathrm{CF}_{2} \bullet$ & -239.88 & -240.30 & -240.12 & -239.98 & -239.98 & -241.47 \\
\hline $\mathrm{CF}_{2} \cdot \mathrm{CF}_{2} \mathrm{C}(=\mathrm{O}) \mathrm{OH}$ & + & $\mathrm{CH}_{3} \mathrm{CH}_{2} \mathrm{~F}$ & $\rightarrow$ & $\mathrm{CHF}_{2} \mathrm{CF}_{2} \mathrm{C}(=\mathrm{O}) \mathrm{OH}$ & + & $\mathrm{CH}_{2} \mathrm{FCH}_{2} \bullet$ & -239.95 & -240.70 & -242.01 & -240.12 & -241.35 & -241.55 \\
\hline $\mathrm{CF}_{2} \cdot \mathrm{CF}_{2} \mathrm{C}(=\mathrm{O}) \mathrm{OH}$ & + & $\mathrm{CH}_{3} \mathrm{CHF}_{2}$ & $\rightarrow$ & $\mathrm{CHF}_{2} \mathrm{CF}_{2} \mathrm{C}(=\mathrm{O}) \mathrm{OH}$ & + & $\mathrm{CHF}_{2} \mathrm{CH}_{2} \bullet$ & -240.17 & -240.79 & -242.24 & -240.34 & -240.90 & -241.42 \\
\hline $\mathrm{CF}_{2} \cdot \mathrm{CF}_{2} \mathrm{C}(=\mathrm{O}) \mathrm{OH}$ & + & $\mathrm{CH}_{2} \mathrm{FCH}_{2} \mathrm{~F}$ & $\rightarrow$ & $\mathrm{CHF}_{2} \mathrm{CF}_{2} \mathrm{C}(=\mathrm{O}) \mathrm{OH}$ & + & $\mathrm{CH}_{2} \mathrm{FCHF} \bullet$ & -239.56 & -239.80 & -239.35 & -239.78 & -240.16 & -241.24 \\
\hline \multirow[t]{4}{*}{$\mathrm{CF}_{2} \cdot \mathrm{CF}_{2} \mathrm{C}(=\mathrm{O}) \mathrm{OH}$} & + & $\mathrm{CH}_{3} \mathrm{CF}_{3}$ & $\rightarrow$ & $\mathrm{CHF}_{2} \mathrm{CF}_{2} \mathrm{C}(=\mathrm{O}) \mathrm{OH}$ & + & $\mathrm{CF}_{3} \mathrm{CH}_{2} \bullet$ & -241.58 & -242.17 & -243.55 & -241.84 & -242.60 & -242.57 \\
\hline & & & & & & Average & -240.24 & -240.80 & -241.40 & -240.31 & -240.89 & -241.76 \\
\hline & & & & & & Uncertainty & 1.17 & 1.54 & 1.19 & 1.21 & 1.11 & 1.16 \\
\hline & & $\mathrm{CH}_{3} \mathrm{CH} \cdot \mathrm{C}(=$ & & & & & & & & & & \\
\hline $\mathrm{CH}_{3} \mathrm{CH} \cdot \mathrm{C}(=\mathrm{O}) \mathrm{OH}$ & + & $\mathrm{CHF}_{2} \mathrm{CHF}_{2}$ & $\rightarrow$ & $\mathrm{CH}_{3} \mathrm{CH}_{2} \mathrm{CO}_{2} \mathrm{H}$ & + & $\mathrm{CHF}_{2} \mathrm{CF}_{2} \bullet$ & -69.67 & -70.58 & -68.74 & -69.62 & -68.91 & -68.59 \\
\hline $\mathrm{CH}_{3} \mathrm{CH} \cdot \mathrm{C}(=\mathrm{O}) \mathrm{OH}$ & + & $\mathrm{CH}_{2} \mathrm{FCHF}_{2}$ & $\rightarrow$ & $\mathrm{CH}_{3} \mathrm{CH}_{2} \mathrm{CO}_{2} \mathrm{H}$ & + & $\mathrm{CH}_{2} \mathrm{FCF}_{2} \bullet$ & -67.97 & -68.61 & -67.03 & -67.87 & -67.16 & -67.15 \\
\hline $\mathrm{CH}_{3} \mathrm{CH} \cdot \mathrm{C}(=\mathrm{O}) \mathrm{OH}$ & + & $\mathrm{CH}_{2} \mathrm{FCHF}_{2}$ & $\rightarrow$ & $\mathrm{CH}_{3} \mathrm{CH}_{2} \mathrm{CO}_{2} \mathrm{H}$ & + & $\mathrm{CHF}_{2} \mathrm{CHF} \bullet$ & -67.46 & -68.35 & -66.97 & -67.54 & -67.16 & -66.85 \\
\hline $\mathrm{CH}_{3} \mathrm{CH} \bullet \mathrm{C}(=\mathrm{O}) \mathrm{OH}$ & + & $\mathrm{CH}_{3} \mathrm{CH}_{2} \mathrm{~F}$ & $\rightarrow$ & $\mathrm{CH}_{3} \mathrm{CH}_{2} \mathrm{CO}_{2} \mathrm{H}$ & + & $\mathrm{CH}_{3} \mathrm{CHF} \bullet$ & -67.38 & -68.69 & -66.71 & -67.10 & -66.93 & -67.29 \\
\hline $\mathrm{CH}_{3} \mathrm{CH} \cdot \mathrm{C}(=\mathrm{O}) \mathrm{OH}$ & + & $\mathrm{CH}_{3} \mathrm{CHF}_{2}$ & $\rightarrow$ & $\mathrm{CH}_{3} \mathrm{CH}_{2} \mathrm{CO}_{2} \mathrm{H}$ & + & $\mathrm{CH}_{3} \mathrm{CF}_{2} \bullet$ & -67.29 & -68.39 & -66.45 & -67.18 & -66.61 & -66.95 \\
\hline $\mathrm{CH}_{3} \mathrm{CH} \cdot \mathrm{C}(=\mathrm{O}) \mathrm{OH}$ & + & $\mathrm{CH}_{2} \mathrm{FCH}_{2} \mathrm{~F}$ & $\rightarrow$ & $\mathrm{CH}_{3} \mathrm{CH}_{2} \mathrm{CO}_{2} \mathrm{H}$ & + & $\mathrm{CH}_{2} \mathrm{FCHF} \bullet$ & -66.98 & -67.89 & -65.68 & -66.98 & -66.79 & -66.72 \\
\hline $\mathrm{CH}_{3} \mathrm{CH} \cdot \mathrm{C}(=\mathrm{O}) \mathrm{OH}$ & + & $\mathrm{CH}_{3} \mathrm{CF}_{3}$ & $\rightarrow$ & $\mathrm{CH}_{3} \mathrm{CH}_{2} \mathrm{CO}_{2} \mathrm{H}$ & + & $\mathrm{CF}_{3} \mathrm{CH}_{2} \bullet$ & -68.99 & -70.26 & -69.88 & -69.04 & -69.23 & -68.05 \\
\hline \multirow[t]{3}{*}{$\mathrm{CH}_{3} \mathrm{CH} \cdot \mathrm{C}(=\mathrm{O}) \mathrm{OH}$} & + & $\mathrm{CH}_{3} \mathrm{CH}_{2} \mathrm{~F}$ & $\rightarrow$ & $\mathrm{CH}_{3} \mathrm{CH}_{2} \mathrm{CO}_{2} \mathrm{H}$ & + & $\mathrm{CH}_{2} \mathrm{FCH}_{2} \bullet$ & -67.37 & -68.79 & -68.35 & -67.32 & -67.98 & -67.03 \\
\hline & & & & & & Average & -67.89 & -68.95 & -67.47 & -67.83 & -67.60 & -67.33 \\
\hline & & & & & & Uncertainty & 1.19 & 1.57 & 1.21 & 1.23 & 1.13 & 1.18 \\
\hline
\end{tabular}




\begin{tabular}{|c|c|c|c|c|c|c|c|c|c|c|c|c|}
\hline \multirow{2}{*}{\multicolumn{7}{|c|}{ Isodesmic reactions }} & \multicolumn{6}{|c|}{$\Delta_{f} \mathbf{H}^{\circ}{ }_{298}\left(\mathrm{kcal} \mathrm{mol}^{-1}\right)$} \\
\hline & & & & & & & \multirow[t]{2}{*}{ M06-2X } & \multirow[t]{2}{*}{ B3LYP } & \multirow[t]{2}{*}{ MN15 } & \multirow[t]{2}{*}{ CBS-QB3 } & \multirow[t]{2}{*}{ CBS-APNO } & \multirow[t]{2}{*}{ G4 } \\
\hline & & $\mathrm{CH}_{2} \mathrm{FCH} \cdot \mathrm{C}($ & & & & & & & & & & \\
\hline $\mathrm{CH}_{2} \mathrm{FCH} \cdot \mathrm{C}(=\mathrm{O}) \mathrm{OH}$ & + & $\mathrm{CH}_{3} \mathrm{CH}_{3}$ & $\rightarrow$ & $\mathrm{CH}_{2} \mathrm{FCH}_{2} \mathrm{C}(=\mathrm{O}) \mathrm{OH}$ & + & $\mathrm{CH}_{3} \mathrm{CH}_{2} \bullet$ & -108.96 & -110.80 & -109.11 & -107.84 & -108.72 & -109.59 \\
\hline $\mathrm{CH}_{2} \mathrm{FCH} \cdot \mathrm{C}(=\mathrm{O}) \mathrm{OH}$ & + & $\mathrm{CH}_{3} \mathrm{CH}_{2} \mathrm{~F}$ & $\rightarrow$ & $\mathrm{CH}_{2} \mathrm{FCH}_{2} \mathrm{C}(=\mathrm{O}) \mathrm{OH}$ & + & $\mathrm{CH}_{3} \mathrm{CHF} \bullet$ & -108.36 & -110.17 & -107.35 & -107.50 & -108.09 & -109.14 \\
\hline $\mathrm{CH}_{2} \mathrm{FCH} \cdot \mathrm{C}(=\mathrm{O}) \mathrm{OH}$ & + & $\mathrm{CH}_{3} \mathrm{CHF}_{2}$ & $\rightarrow$ & $\mathrm{CH}_{2} \mathrm{FCH}_{2} \mathrm{C}(=\mathrm{O}) \mathrm{OH}$ & + & $\mathrm{CH}_{3} \mathrm{CF}_{2} \bullet$ & -108.27 & -109.87 & -107.10 & -107.58 & -107.77 & -108.80 \\
\hline $\mathrm{CH}_{2} \mathrm{FCH} \cdot \mathrm{C}(=\mathrm{O}) \mathrm{OH}$ & + & $\mathrm{CH}_{3} \mathrm{CH}_{2} \mathrm{~F}$ & $\rightarrow$ & $\mathrm{CH}_{2} \mathrm{FCH}_{2} \mathrm{C}(=\mathrm{O}) \mathrm{OH}$ & + & $\mathrm{CH}_{2} \mathrm{FCH}_{2} \bullet$ & -108.73 & -110.66 & -109.38 & -108.10 & -109.52 & -109.27 \\
\hline $\mathrm{CH}_{2} \mathrm{FCH} \cdot \mathrm{C}(=\mathrm{O}) \mathrm{OH}$ & + & $\mathrm{CH}_{3} \mathrm{CHF}_{2}$ & $\rightarrow$ & $\mathrm{CH}_{2} \mathrm{FCH}_{2} \mathrm{C}(=\mathrm{O}) \mathrm{OH}$ & + & $\mathrm{CHF}_{2} \mathrm{CH}_{2} \bullet$ & -108.95 & -110.74 & -109.61 & -108.32 & -109.08 & -109.13 \\
\hline $\mathrm{CH}_{2} \mathrm{FCH} \cdot \mathrm{C}(=\mathrm{O}) \mathrm{OH}$ & + & $\mathrm{CH}_{2} \mathrm{FCH}_{2} \mathrm{~F}$ & $\rightarrow$ & $\mathrm{CH}_{2} \mathrm{FCH}_{2} \mathrm{C}(=\mathrm{O}) \mathrm{OH}$ & + & $\mathrm{CH}_{2} \mathrm{FCHF} \bullet$ & -108.34 & -109.76 & -106.71 & -107.77 & -108.34 & -108.95 \\
\hline \multirow[t]{3}{*}{$\mathrm{CH}_{2} \mathrm{FCH} \cdot \mathrm{C}(=\mathrm{O}) \mathrm{OH}$} & + & $\mathrm{CH}_{3} \mathrm{CF}_{3}$ & $\rightarrow$ & $\mathrm{CH}_{2} \mathrm{FCH}_{2} \mathrm{C}(=\mathrm{O}) \mathrm{OH}$ & + & $\mathrm{CF}_{3} \mathrm{CH}_{2} \bullet$ & -110.36 & -112.12 & -110.91 & -109.82 & -110.77 & -110.28 \\
\hline & & & & & & Average & -108.85 & -110.59 & -108.60 & -108.13 & -108.90 & -109.31 \\
\hline & & & & & & Uncertainty & 1.12 & 1.50 & 1.14 & 1.16 & 1.06 & 1.11 \\
\hline & & $\mathrm{CHF}_{2} \mathrm{CH} \cdot \mathrm{C}($ & & & & & & & & & & \\
\hline $\mathrm{CHF}_{2} \mathrm{CH} \cdot \mathrm{C}(=\mathrm{O}) \mathrm{OH}$ & + & $\mathrm{CH}_{3} \mathrm{CH}_{3}$ & $\rightarrow$ & $\mathrm{CHF}_{2} \mathrm{CH}_{2} \mathrm{C}(=\mathrm{O}) \mathrm{OH}$ & + & $\mathrm{CH}_{3} \mathrm{CH}_{2} \bullet$ & -160.12 & -161.71 & -160.26 & -159.74 & -159.88 & -160.85 \\
\hline $\mathrm{CHF}_{2} \mathrm{CH} \cdot \mathrm{C}(=\mathrm{O}) \mathrm{OH}$ & + & $\mathrm{CH}_{3} \mathrm{CH}_{2} \mathrm{~F}$ & $\rightarrow$ & $\mathrm{CHF}_{2} \mathrm{CH}_{2} \mathrm{C}(=\mathrm{O}) \mathrm{OH}$ & + & $\mathrm{CH}_{3} \mathrm{CHF} \bullet$ & -159.52 & -161.08 & -158.50 & -159.39 & -159.24 & -160.39 \\
\hline $\mathrm{CHF}_{2} \mathrm{CH} \cdot \mathrm{C}(=\mathrm{O}) \mathrm{OH}$ & + & $\mathrm{CH}_{3} \mathrm{CHF}_{2}$ & $\rightarrow$ & $\mathrm{CHF}_{2} \mathrm{CH}_{2} \mathrm{C}(=\mathrm{O}) \mathrm{OH}$ & + & $\mathrm{CH}_{3} \mathrm{CF}_{2} \bullet$ & -159.43 & -160.78 & -158.24 & -159.48 & -158.92 & -160.05 \\
\hline $\mathrm{CHF}_{2} \mathrm{CH} \cdot \mathrm{C}(=\mathrm{O}) \mathrm{OH}$ & + & $\mathrm{CH}_{3} \mathrm{CH}_{2} \mathrm{~F}$ & $\rightarrow$ & $\mathrm{CHF}_{2} \mathrm{CH}_{2} \mathrm{C}(=\mathrm{O}) \mathrm{OH}$ & + & $\mathrm{CH}_{2} \mathrm{FCH}_{2} \bullet$ & -159.51 & -161.19 & -160.14 & -159.61 & -160.29 & -160.13 \\
\hline $\mathrm{CHF}_{2} \mathrm{CH} \cdot \mathrm{C}(=\mathrm{O}) \mathrm{OH}$ & + & $\mathrm{CH}_{3} \mathrm{CHF}_{2}$ & $\rightarrow$ & $\mathrm{CHF}_{2} \mathrm{CH}_{2} \mathrm{C}(=\mathrm{O}) \mathrm{OH}$ & + & $\mathrm{CHF}_{2} \mathrm{CH}_{2} \bullet$ & -159.72 & -161.27 & -160.37 & -159.83 & -159.85 & -160.00 \\
\hline $\mathrm{CHF}_{2} \mathrm{CH} \cdot \mathrm{C}(=\mathrm{O}) \mathrm{OH}$ & + & $\mathrm{CH}_{2} \mathrm{FCH}_{2} \mathrm{~F}$ & $\rightarrow$ & $\mathrm{CHF}_{2} \mathrm{CH}_{2} \mathrm{C}(=\mathrm{O}) \mathrm{OH}$ & + & $\mathrm{CH}_{2} \mathrm{FCHF} \bullet$ & -159.12 & -160.28 & -157.47 & -159.28 & -159.11 & -159.82 \\
\hline \multirow[t]{3}{*}{$\mathrm{CHF}_{2} \mathrm{CH} \cdot \mathrm{C}(=\mathrm{O}) \mathrm{OH}$} & + & $\mathrm{CH}_{3} \mathrm{CF}_{3}$ & $\rightarrow$ & $\mathrm{CHF}_{2} \mathrm{CH}_{2} \mathrm{C}(=\mathrm{O}) \mathrm{OH}$ & + & $\mathrm{CF}_{3} \mathrm{CH}_{2} \bullet$ & -161.13 & -162.65 & -161.67 & -161.34 & -161.55 & -161.15 \\
\hline & & & & & & Average & -159.79 & -161.28 & -159.52 & -159.81 & -159.83 & -160.34 \\
\hline & & & & & & Uncertainty & 1.00 & 1.41 & 1.03 & 1.05 & 0.94 & 0.99 \\
\hline
\end{tabular}




\begin{tabular}{|c|c|c|c|c|c|c|c|c|c|c|c|c|}
\hline \multirow{2}{*}{\multicolumn{7}{|c|}{ Isodesmic reactions }} & \multicolumn{6}{|c|}{$\Delta f \mathbf{H}^{\circ}{ }_{298}\left(\mathrm{kcal} \mathrm{mol}^{-1}\right)$} \\
\hline & & & & & & & \multirow[t]{2}{*}{ M06-2X } & \multirow[t]{2}{*}{ B3LYP } & \multirow[t]{2}{*}{ MN15 } & \multirow[t]{2}{*}{ CBS-QB3 } & \multirow[t]{2}{*}{ CBS-APNO } & \multirow[t]{2}{*}{ G4 } \\
\hline & & $\mathrm{CF}_{3} \mathrm{CH} \cdot \mathrm{C}(=$ & & & & & & & & & & \\
\hline $\mathrm{CF}_{3} \mathrm{CH} \cdot \mathrm{C}(=\mathrm{O}) \mathrm{OH}$ & + & $\mathrm{CH}_{3} \mathrm{CH}_{3}$ & $\rightarrow$ & $\mathrm{CF}_{3} \mathrm{CH}_{2} \mathrm{C}(=\mathrm{O}) \mathrm{OH}$ & + & $\mathrm{CH}_{3} \mathrm{CH}_{2} \bullet$ & -214.78 & -216.25 & -214.99 & -214.37 & -214.57 & -215.91 \\
\hline $\mathrm{CF}_{3} \mathrm{CH} \cdot \mathrm{C}(=\mathrm{O}) \mathrm{OH}$ & + & $\mathrm{CH}_{3} \mathrm{CH}_{2} \mathrm{~F}$ & $\rightarrow$ & $\mathrm{CF}_{3} \mathrm{CH}_{2} \mathrm{C}(=\mathrm{O}) \mathrm{OH}$ & + & $\mathrm{CH}_{3} \mathrm{CHF} \bullet$ & -214.18 & -215.62 & -213.23 & -214.02 & -213.93 & -215.45 \\
\hline $\mathrm{CF}_{3} \mathrm{CH} \cdot \mathrm{C}(=\mathrm{O}) \mathrm{OH}$ & + & $\mathrm{CH}_{3} \mathrm{CHF}_{2}$ & $\rightarrow$ & $\mathrm{CF}_{3} \mathrm{CH}_{2} \mathrm{C}(=\mathrm{O}) \mathrm{OH}$ & + & $\mathrm{CH}_{3} \mathrm{CF}_{2} \bullet$ & -214.09 & -215.32 & -212.98 & -214.11 & -213.61 & -215.11 \\
\hline $\mathrm{CF}_{3} \mathrm{CH} \cdot \mathrm{C}(=\mathrm{O}) \mathrm{OH}$ & + & $\mathrm{CH}_{3} \mathrm{CH}_{2} \mathrm{~F}$ & $\rightarrow$ & $\mathrm{CF}_{3} \mathrm{CH}_{2} \mathrm{C}(=\mathrm{O}) \mathrm{OH}$ & + & $\mathrm{CH}_{2} \mathrm{FCH}_{2} \bullet$ & -214.17 & -215.72 & -214.87 & -214.24 & -214.98 & -215.20 \\
\hline $\mathrm{CF}_{3} \mathrm{CH} \cdot \mathrm{C}(=\mathrm{O}) \mathrm{OH}$ & + & $\mathrm{CH}_{3} \mathrm{CHF}_{2}$ & $\rightarrow$ & $\mathrm{CF}_{3} \mathrm{CH}_{2} \mathrm{C}(=\mathrm{O}) \mathrm{OH}$ & + & $\mathrm{CHF}_{2} \mathrm{CH}_{2} \bullet$ & -214.38 & -215.80 & -215.10 & -214.46 & -214.54 & -215.06 \\
\hline $\mathrm{CF}_{3} \mathrm{CH} \cdot \mathrm{C}(=\mathrm{O}) \mathrm{OH}$ & + & $\mathrm{CH}_{2} \mathrm{FCH}_{2} \mathrm{~F}$ & $\rightarrow$ & $\mathrm{CF}_{3} \mathrm{CH}_{2} \mathrm{C}(=\mathrm{O}) \mathrm{OH}$ & + & $\mathrm{CH}_{2} \mathrm{FCHF} \bullet$ & -213.78 & -214.82 & -212.20 & -213.91 & -213.80 & -214.88 \\
\hline \multirow[t]{4}{*}{$\mathrm{CF}_{3} \mathrm{CH} \cdot \mathrm{C}(=\mathrm{O}) \mathrm{OH}$} & + & $\mathrm{CH}_{3} \mathrm{CF}_{3}$ & $\rightarrow$ & $\mathrm{CF}_{3} \mathrm{CH}_{2} \mathrm{C}(=\mathrm{O}) \mathrm{OH}$ & + & $\mathrm{CF}_{3} \mathrm{CH}_{2} \bullet$ & -215.79 & -217.18 & -216.41 & -215.97 & -216.24 & -216.21 \\
\hline & & & & & & Average & -214.45 & -215.82 & -214.26 & -214.44 & -214.52 & -215.40 \\
\hline & & & & & & Uncertainty & 1.05 & 1.45 & 1.07 & 1.10 & 0.99 & 1.04 \\
\hline & & $\mathrm{CH}_{3} \mathrm{CF} \cdot \mathrm{C}(=$ & & & & & & & & & & \\
\hline $\mathrm{CH}_{3} \mathrm{CF} \cdot \mathrm{C}(=\mathrm{O}) \mathrm{OH}$ & + & $\mathrm{CH}_{3} \mathrm{CH}_{3}$ & $\rightarrow$ & $\mathrm{CH}_{3} \mathrm{CHFC}(=\mathrm{O}) \mathrm{OH}$ & + & $\mathrm{CH}_{3} \mathrm{CH}_{2} \cdot$ & -115.18 & -116.53 & -116.91 & -114.49 & -114.91 & -114.59 \\
\hline $\mathrm{CH}_{3} \mathrm{CF} \cdot \mathrm{C}(=\mathrm{O}) \mathrm{OH}$ & + & $\mathrm{CH}_{3} \mathrm{CH}_{2} \mathrm{~F}$ & $\rightarrow$ & $\mathrm{CH}_{3} \mathrm{CHFC}(=\mathrm{O}) \mathrm{OH}$ & + & $\mathrm{CH}_{3} \mathrm{CHF} \bullet$ & -114.58 & -115.90 & -115.15 & -114.15 & -114.27 & -114.13 \\
\hline $\mathrm{CH}_{3} \mathrm{CF} \cdot \mathrm{C}(=\mathrm{O}) \mathrm{OH}$ & + & $\mathrm{CH}_{3} \mathrm{CHF}_{2}$ & $\rightarrow$ & $\mathrm{CH}_{3} \mathrm{CHFC}(=\mathrm{O}) \mathrm{OH}$ & + & $\mathrm{CH}_{3} \mathrm{CF}_{2} \bullet$ & -114.49 & -115.60 & -114.89 & -114.23 & -113.96 & -113.79 \\
\hline $\mathrm{CH}_{3} \mathrm{CF} \cdot \mathrm{C}(=\mathrm{O}) \mathrm{OH}$ & + & $\mathrm{CH}_{3} \mathrm{CH}_{2} \mathrm{~F}$ & $\rightarrow$ & $\mathrm{CH}_{3} \mathrm{CHFC}(=\mathrm{O}) \mathrm{OH}$ & + & $\mathrm{CH}_{2} \mathrm{FCH}_{2} \bullet$ & -114.57 & -116.00 & -116.79 & -114.37 & -115.32 & -113.88 \\
\hline $\mathrm{CH}_{3} \mathrm{CF} \cdot \mathrm{C}(=\mathrm{O}) \mathrm{OH}$ & + & $\mathrm{CH}_{3} \mathrm{CHF}_{2}$ & $\rightarrow$ & $\mathrm{CH}_{3} \mathrm{CHFC}(=\mathrm{O}) \mathrm{OH}$ & + & $\mathrm{CHF}_{2} \mathrm{CH}_{2} \bullet$ & -114.78 & -116.08 & -117.02 & -114.59 & -114.88 & -113.74 \\
\hline $\mathrm{CH}_{3} \mathrm{CF} \cdot \mathrm{C}(=\mathrm{O}) \mathrm{OH}$ & + & $\mathrm{CH}_{2} \mathrm{FCH}_{2} \mathrm{~F}$ & $\rightarrow$ & $\mathrm{CH}_{3} \mathrm{CHFC}(=\mathrm{O}) \mathrm{OH}$ & + & $\mathrm{CH}_{2} \mathrm{FCHF} \bullet$ & -114.18 & -115.10 & -114.12 & -114.04 & -114.14 & -113.56 \\
\hline \multirow[t]{3}{*}{$\mathrm{CH}_{3} \mathrm{CF} \cdot \mathrm{C}(=\mathrm{O}) \mathrm{OH}$} & + & $\mathrm{CH}_{3} \mathrm{CF}_{3}$ & $\rightarrow$ & $\mathrm{CH}_{3} \mathrm{CHFC}(=\mathrm{O}) \mathrm{OH}$ & + & $\mathrm{CF}_{3} \mathrm{CH}_{2} \bullet$ & -116.19 & -117.46 & -118.33 & -116.09 & -116.58 & -114.89 \\
\hline & & & & & & Average & -114.85 & -116.10 & -116.17 & -114.57 & -114.87 & -114.08 \\
\hline & & & & & & Uncertainty & 1.12 & 1.50 & 1.14 & 1.17 & 1.06 & 1.11 \\
\hline
\end{tabular}




\begin{tabular}{|c|c|c|c|c|c|c|c|c|c|c|c|c|}
\hline \multirow{2}{*}{\multicolumn{7}{|c|}{ Isodesmic reactions }} & \multicolumn{6}{|c|}{$\Delta_{f} \mathrm{H}^{\circ}{ }_{298}\left(\mathrm{kcal} \mathrm{mol}^{-1}\right)$} \\
\hline & & & & & & & \multirow[t]{2}{*}{ M06-2X } & \multirow[t]{2}{*}{ B3LYP } & \multirow[t]{2}{*}{ MN15 } & \multirow[t]{2}{*}{ CBS-QB3 } & \multirow[t]{2}{*}{ CBS-APNO } & \multirow[t]{2}{*}{ G4 } \\
\hline & & $\mathrm{CH}_{2} \mathrm{FCF} \cdot \mathrm{C}($ & & & & & & & & & & \\
\hline $\mathrm{CH}_{2} \mathrm{FCF} \cdot \mathrm{C}(=\mathrm{O}) \mathrm{OH}$ & + & $\mathrm{CH}_{3} \mathrm{CH}_{3}$ & $\rightarrow$ & $\mathrm{CH}_{2} \mathrm{FCHFC}(=\mathrm{O}) \mathrm{OH}$ & + & $\mathrm{CH}_{3} \mathrm{CH}_{2} \bullet$ & -153.43 & -155.47 & -154.81 & -153.00 & -153.56 & -153.39 \\
\hline $\mathrm{CH}_{2} \mathrm{FCF} \cdot \mathrm{C}(=\mathrm{O}) \mathrm{OH}$ & + & $\mathrm{CH}_{3} \mathrm{CH}_{2} \mathrm{~F}$ & $\rightarrow$ & $\mathrm{CH}_{2} \mathrm{FCHFC}(=\mathrm{O}) \mathrm{OH}$ & + & $\mathrm{CH}_{3} \mathrm{CHF} \bullet$ & -152.83 & -154.84 & -153.05 & -152.65 & -152.92 & -152.93 \\
\hline $\mathrm{CH}_{2} \mathrm{FCF} \cdot \mathrm{C}(=\mathrm{O}) \mathrm{OH}$ & + & $\mathrm{CH}_{3} \mathrm{CHF}_{2}$ & $\rightarrow$ & $\mathrm{CH}_{2} \mathrm{FCHFC}(=\mathrm{O}) \mathrm{OH}$ & + & $\mathrm{CH}_{3} \mathrm{CF}_{2} \bullet$ & -152.74 & -154.54 & -152.79 & -152.74 & -152.60 & -152.59 \\
\hline $\mathrm{CH}_{2} \mathrm{FCF} \cdot \mathrm{C}(=\mathrm{O}) \mathrm{OH}$ & + & $\mathrm{CH}_{3} \mathrm{CH}_{2} \mathrm{~F}$ & $\rightarrow$ & $\mathrm{CH}_{2} \mathrm{FCHFC}(=\mathrm{O}) \mathrm{OH}$ & + & $\mathrm{CH}_{2} \mathrm{FCH}_{2} \bullet$ & -152.82 & -154.95 & -154.69 & -152.88 & -153.97 & -152.67 \\
\hline $\mathrm{CH}_{2} \mathrm{FCF} \cdot \mathrm{C}(=\mathrm{O}) \mathrm{OH}$ & + & $\mathrm{CH}_{3} \mathrm{CHF}_{2}$ & $\rightarrow$ & $\mathrm{CH}_{2} \mathrm{FCHFC}(=\mathrm{O}) \mathrm{OH}$ & + & $\mathrm{CHF}_{2} \mathrm{CH}_{2} \bullet$ & -153.03 & -155.03 & -154.92 & -153.09 & -153.53 & -152.54 \\
\hline $\mathrm{CH}_{2} \mathrm{FCF} \cdot \mathrm{C}(=\mathrm{O}) \mathrm{OH}$ & + & $\mathrm{CH}_{2} \mathrm{FCH}_{2} \mathrm{~F}$ & $\rightarrow$ & $\mathrm{CH}_{2} \mathrm{FCHFC}(=\mathrm{O}) \mathrm{OH}$ & + & $\mathrm{CH}_{2} \mathrm{FCHF} \bullet$ & -152.43 & -154.05 & -152.02 & -152.54 & -152.79 & -152.36 \\
\hline \multirow[t]{4}{*}{$\mathrm{CH}_{2} \mathrm{FCF} \cdot \mathrm{C}(=\mathrm{O}) \mathrm{OH}$} & + & $\mathrm{CH}_{3} \mathrm{CF}_{3}$ & $\rightarrow$ & $\mathrm{CH}_{2} \mathrm{FCHFC}(=\mathrm{O}) \mathrm{OH}$ & + & $\mathrm{CF}_{3} \mathrm{CH}_{2} \bullet$ & -154.44 & -156.41 & -156.22 & -154.60 & -155.22 & -153.69 \\
\hline & & & & & & Average & -153.10 & -155.04 & -154.07 & -153.07 & -153.51 & -152.88 \\
\hline & & & & & & Uncertainty & 1.00 & 1.41 & 1.03 & 1.05 & 0.94 & 0.99 \\
\hline & & $\mathrm{CHF}_{2} \mathrm{CF} \cdot \mathrm{C}($ & & & & & & & & & & \\
\hline $\mathrm{CHF}_{2} \mathrm{CF} \cdot \mathrm{C}(=\mathrm{O}) \mathrm{OH}$ & + & $\mathrm{CH}_{3} \mathrm{CH}_{3}$ & $\rightarrow$ & $\mathrm{CHF}_{2} \mathrm{CHFC}(=\mathrm{O}) \mathrm{OH}$ & + & $\mathrm{CH}_{3} \mathrm{CH}_{2} \bullet$ & -202.25 & -204.27 & -204.45 & -201.83 & -202.44 & -202.72 \\
\hline $\mathrm{CHF}_{2} \mathrm{CF} \cdot \mathrm{C}(=\mathrm{O}) \mathrm{OH}$ & + & $\mathrm{CH}_{3} \mathrm{CH}_{2} \mathrm{~F}$ & $\rightarrow$ & $\mathrm{CHF}_{2} \mathrm{CHFC}(=\mathrm{O}) \mathrm{OH}$ & + & $\mathrm{CH}_{3} \mathrm{CHF} \bullet$ & -201.65 & -203.64 & -202.69 & -201.48 & -201.81 & -202.26 \\
\hline $\mathrm{CHF}_{2} \mathrm{CF} \cdot \mathrm{C}(=\mathrm{O}) \mathrm{OH}$ & + & $\mathrm{CH}_{3} \mathrm{CHF}_{2}$ & $\rightarrow$ & $\mathrm{CHF}_{2} \mathrm{CHFC}(=\mathrm{O}) \mathrm{OH}$ & + & $\mathrm{CH}_{3} \mathrm{CF}_{2} \bullet$ & -201.56 & -203.34 & -202.44 & -201.57 & -201.49 & -201.92 \\
\hline $\mathrm{CHF}_{2} \mathrm{CF} \cdot \mathrm{C}(=\mathrm{O}) \mathrm{OH}$ & + & $\mathrm{CH}_{3} \mathrm{CH}_{2} \mathrm{~F}$ & $\rightarrow$ & $\mathrm{CHF}_{2} \mathrm{CHFC}(=\mathrm{O}) \mathrm{OH}$ & + & $\mathrm{CH}_{2} \mathrm{FCH}_{2} \bullet$ & -201.64 & -203.74 & -204.33 & -201.71 & -202.86 & -202.01 \\
\hline $\mathrm{CHF}_{2} \mathrm{CF} \cdot \mathrm{C}(=\mathrm{O}) \mathrm{OH}$ & + & $\mathrm{CH}_{3} \mathrm{CHF}_{2}$ & $\rightarrow$ & $\mathrm{CHF}_{2} \mathrm{CHFC}(=\mathrm{O}) \mathrm{OH}$ & + & $\mathrm{CHF}_{2} \mathrm{CH}_{2} \bullet$ & -201.85 & -203.83 & -204.56 & -201.92 & -202.41 & -201.87 \\
\hline $\mathrm{CHF}_{2} \mathrm{CF} \cdot \mathrm{C}(=\mathrm{O}) \mathrm{OH}$ & + & $\mathrm{CH}_{2} \mathrm{FCH}_{2} \mathrm{~F}$ & $\rightarrow$ & $\mathrm{CHF}_{2} \mathrm{CHFC}(=\mathrm{O}) \mathrm{OH}$ & + & $\mathrm{CH}_{2} \mathrm{FCHF} \bullet$ & -201.25 & -202.84 & -201.66 & -201.37 & -201.67 & -201.70 \\
\hline \multirow[t]{3}{*}{$\mathrm{CHF}_{2} \mathrm{CF} \cdot \mathrm{C}(=\mathrm{O}) \mathrm{OH}$} & + & $\mathrm{CH}_{3} \mathrm{CF}_{3}$ & $\rightarrow$ & $\mathrm{CHF}_{2} \mathrm{CHFC}(=\mathrm{O}) \mathrm{OH}$ & + & $\mathrm{CF}_{3} \mathrm{CH}_{2} \bullet$ & -203.26 & -205.20 & -205.87 & -203.43 & -204.11 & -203.02 \\
\hline & & & & & & Average & -201.92 & -203.84 & -203.72 & -201.90 & -202.40 & -202.22 \\
\hline & & & & & & Uncertainty & 0.94 & 1.38 & 0.97 & 1.00 & 0.86 & 0.93 \\
\hline
\end{tabular}




\begin{tabular}{|c|c|c|c|c|c|c|c|c|c|c|c|c|}
\hline \multirow{2}{*}{\multicolumn{7}{|c|}{ Isodesmic reactions }} & \multicolumn{6}{|c|}{$\Delta_{f} \mathrm{H}^{\circ}{ }_{298}\left(\mathrm{kcal} \mathrm{mol}^{-1}\right)$} \\
\hline & & & & & & & \multirow[t]{2}{*}{ M06-2X } & \multirow[t]{2}{*}{ B3LYP } & \multirow[t]{2}{*}{ MN15 } & \multirow[t]{2}{*}{ CBS-QB3 } & \multirow[t]{2}{*}{ CBS-APNO } & \multirow[t]{2}{*}{ G4 } \\
\hline & & $\mathrm{CF}_{3} \mathrm{CF} \cdot \mathrm{C}($ & & & & & & & & & & \\
\hline $\mathrm{CF}_{3} \mathrm{CF} \cdot \mathrm{C}(=\mathrm{O}) \mathrm{OH}$ & + & $\mathrm{CH}_{3} \mathrm{CH}_{3}$ & $\rightarrow$ & $\mathrm{CF}_{3} \mathrm{CHFC}(=\mathrm{O}) \mathrm{OH}$ & + & $\mathrm{CH}_{3} \mathrm{CH}_{2} \bullet$ & -256.66 & -258.00 & -258.16 & -255.45 & -256.62 & -256.72 \\
\hline $\mathrm{CF}_{3} \mathrm{CF} \cdot \mathrm{C}(=\mathrm{O}) \mathrm{OH}$ & + & $\mathrm{CH}_{3} \mathrm{CH}_{2} \mathrm{~F}$ & $\rightarrow$ & $\mathrm{CF}_{3} \mathrm{CHFC}(=\mathrm{O}) \mathrm{OH}$ & + & $\mathrm{CH}_{3} \mathrm{CHF} \bullet$ & -256.06 & -257.37 & -256.40 & -255.11 & -255.98 & -256.26 \\
\hline $\mathrm{CF}_{3} \mathrm{CF} \cdot \mathrm{C}(=\mathrm{O}) \mathrm{OH}$ & + & $\mathrm{CH}_{3} \mathrm{CHF}_{2}$ & $\rightarrow$ & $\mathrm{CF}_{3} \mathrm{CHFC}(=\mathrm{O}) \mathrm{OH}$ & + & $\mathrm{CH}_{3} \mathrm{CF}_{2} \bullet$ & -255.97 & -257.07 & -256.15 & -255.19 & -255.66 & -255.93 \\
\hline $\mathrm{CF}_{3} \mathrm{CF} \cdot \mathrm{C}(=\mathrm{O}) \mathrm{OH}$ & + & $\mathrm{CH}_{3} \mathrm{CH}_{2} \mathrm{~F}$ & $\rightarrow$ & $\mathrm{CF}_{3} \mathrm{CHFC}(=\mathrm{O}) \mathrm{OH}$ & + & $\mathrm{CH}_{2} \mathrm{FCH}_{2} \bullet$ & -256.05 & -257.48 & -258.04 & -255.33 & -257.03 & -256.01 \\
\hline $\mathrm{CF}_{3} \mathrm{CF} \cdot \mathrm{C}(=\mathrm{O}) \mathrm{OH}$ & + & $\mathrm{CH}_{3} \mathrm{CHF}_{2}$ & $\rightarrow$ & $\mathrm{CF}_{3} \mathrm{CHFC}(=\mathrm{O}) \mathrm{OH}$ & + & $\mathrm{CHF}_{2} \mathrm{CH}_{2} \bullet$ & -256.26 & -257.56 & -258.27 & -255.55 & -256.59 & -255.87 \\
\hline $\mathrm{CF}_{3} \mathrm{CF} \cdot \mathrm{C}(=\mathrm{O}) \mathrm{OH}$ & + & $\mathrm{CH}_{2} \mathrm{FCH}_{2} \mathrm{~F}$ & $\rightarrow$ & $\mathrm{CF}_{3} \mathrm{CHFC}(=\mathrm{O}) \mathrm{OH}$ & + & $\mathrm{CH}_{2} \mathrm{FCHF} \bullet$ & -255.66 & -256.57 & -255.37 & -254.99 & -255.85 & -255.70 \\
\hline \multirow[t]{3}{*}{$\mathrm{CF}_{3} \mathrm{CF} \cdot \mathrm{C}(=\mathrm{O}) \mathrm{OH}$} & + & $\mathrm{CH}_{3} \mathrm{CF}_{3}$ & $\rightarrow$ & $\mathrm{CF}_{3} \mathrm{CHFC}(=\mathrm{O}) \mathrm{OH}$ & + & $\mathrm{CF}_{3} \mathrm{CH}_{2} \bullet$ & -257.67 & -258.94 & -259.58 & -257.05 & -258.29 & -257.03 \\
\hline & & & & & & Average & -256.33 & -257.57 & -257.42 & -255.52 & -256.57 & -256.22 \\
\hline & & & & & & Uncertainty & 1.20 & 1.56 & 1.21 & 1.24 & 1.14 & 1.18 \\
\hline & & $\mathrm{CH}_{3} \mathrm{CH}_{2} \mathrm{C}$ & & & & & & & & & & \\
\hline $\mathrm{CH}_{3} \mathrm{CH}_{2} \mathrm{C}(=\mathrm{O}) \mathrm{O} \bullet$ & + & $\mathrm{CH}_{3} \mathrm{CH}_{3}$ & $\rightarrow$ & $\mathrm{CH}_{3} \mathrm{CH}_{2} \mathrm{C}(=\mathrm{O}) \mathrm{OH}$ & + & $\mathrm{CH}_{3} \mathrm{CH}_{2} \bullet$ & -47.71 & -54.93 & -48.24 & -51.42 & - & -51.23 \\
\hline $\mathrm{CH}_{3} \mathrm{CH}_{2} \mathrm{C}(=\mathrm{O}) \mathrm{O} \bullet$ & + & $\mathrm{CH}_{3} \mathrm{CH}_{2} \mathrm{~F}$ & $\rightarrow$ & $\mathrm{CH}_{3} \mathrm{CH}_{2} \mathrm{C}(=\mathrm{O}) \mathrm{OH}$ & + & $\mathrm{CH}_{3} \mathrm{CHF} \bullet$ & -47.11 & -54.30 & -46.48 & -51.08 & - & -50.77 \\
\hline $\mathrm{CH}_{3} \mathrm{CH}_{2} \mathrm{C}(=\mathrm{O}) \mathrm{O} \bullet$ & + & $\mathrm{CH}_{3} \mathrm{CHF}_{2}$ & $\rightarrow$ & $\mathrm{CH}_{3} \mathrm{CH}_{2} \mathrm{C}(=\mathrm{O}) \mathrm{OH}$ & + & $\mathrm{CH}_{3} \mathrm{CF}_{2} \bullet$ & -47.02 & -54.00 & -46.22 & -51.16 & - & -50.43 \\
\hline $\mathrm{CH}_{3} \mathrm{CH}_{2} \mathrm{C}(=\mathrm{O}) \mathrm{O} \bullet$ & + & $\mathrm{CH}_{3} \mathrm{CH}_{2} \mathrm{~F}$ & $\rightarrow$ & $\mathrm{CH}_{3} \mathrm{CH}_{2} \mathrm{C}(=\mathrm{O}) \mathrm{OH}$ & + & $\mathrm{CH}_{2} \mathrm{FCH}_{2} \bullet$ & -47.10 & -54.41 & -48.12 & -51.30 & - & -50.51 \\
\hline $\mathrm{CH}_{3} \mathrm{CH}_{2} \mathrm{C}(=\mathrm{O}) \mathrm{O} \bullet$ & + & $\mathrm{CH}_{3} \mathrm{CHF}_{2}$ & $\rightarrow$ & $\mathrm{CH}_{3} \mathrm{CH}_{2} \mathrm{C}(=\mathrm{O}) \mathrm{OH}$ & + & $\mathrm{CHF}_{2} \mathrm{CH}_{2} \bullet$ & -47.31 & -54.49 & -48.35 & -51.52 & - & -50.38 \\
\hline $\mathrm{CH}_{3} \mathrm{CH}_{2} \mathrm{C}(=\mathrm{O}) \mathrm{O} \bullet$ & + & $\mathrm{CH}_{2} \mathrm{FCH}_{2} \mathrm{~F}$ & $\rightarrow$ & $\mathrm{CH}_{3} \mathrm{CH}_{2} \mathrm{C}(=\mathrm{O}) \mathrm{OH}$ & + & $\mathrm{CH}_{2} \mathrm{FCHF} \bullet$ & -46.71 & -53.50 & -45.45 & -50.96 & - & -50.20 \\
\hline \multirow[t]{3}{*}{$\mathrm{CH}_{3} \mathrm{CH}_{2} \mathrm{C}(=\mathrm{O}) \mathrm{O} \bullet$} & + & $\mathrm{CH}_{3} \mathrm{CF}_{3}$ & $\rightarrow$ & $\mathrm{CH}_{3} \mathrm{CH}_{2} \mathrm{C}(=\mathrm{O}) \mathrm{OH}$ & + & $\mathrm{CF}_{3} \mathrm{CH}_{2} \bullet$ & -48.72 & -55.87 & -49.65 & -53.02 & - & -51.53 \\
\hline & & & & & & Average & -47.38 & -54.50 & -47.50 & -51.49 & - & -50.72 \\
\hline & & & & & & Uncertainty & 0.81 & 1.29 & 0.84 & 0.87 & - & 0.80 \\
\hline
\end{tabular}




\begin{tabular}{|c|c|c|c|c|c|c|c|c|c|c|c|}
\hline \multirow{2}{*}{\multicolumn{6}{|c|}{ Isodesmic reactions }} & \multicolumn{6}{|c|}{$\Delta_{f} \mathbf{H}^{\circ}{ }_{298}\left(\mathrm{kcal} \mathrm{mol}^{-1}\right)$} \\
\hline & & & & & & \multirow[t]{2}{*}{ M06-2X } & \multirow[t]{2}{*}{ B3LYP } & \multirow[t]{2}{*}{ MN15 } & \multirow[t]{2}{*}{ CBS-QB3 } & \multirow[t]{2}{*}{ CBS-APNO } & \multirow[t]{2}{*}{ G4 } \\
\hline & & $\mathrm{CH}_{3} \mathrm{CHFC}$ & & & & & & & & & \\
\hline $\mathrm{CH}_{3} \mathrm{CHFC}(=\mathrm{O}) \mathrm{O} \bullet$ & + & $\mathrm{CH}_{3} \mathrm{CH}_{3}$ & $\rightarrow$ & $\mathrm{CH}_{3} \mathrm{CHFC}(=\mathrm{O}) \mathrm{OH}$ & $\mathrm{CH}_{3} \mathrm{CH}_{2} \bullet$ & -90.57 & -97.06 & -90.04 & -93.73 & -91.73 & -93.85 \\
\hline $\mathrm{CH}_{3} \mathrm{CHFC}(=\mathrm{O}) \mathrm{O} \bullet$ & + & $\mathrm{CH}_{3} \mathrm{CH}_{2} \mathrm{~F}$ & $\rightarrow$ & $\mathrm{CH}_{3} \mathrm{CHFC}(=\mathrm{O}) \mathrm{OH}$ & $\mathrm{CH}_{3} \mathrm{CHF} \bullet$ & -89.97 & -96.43 & -88.28 & -93.38 & -91.10 & -93.39 \\
\hline $\mathrm{CH}_{3} \mathrm{CHFC}(=\mathrm{O}) \mathrm{O} \bullet$ & + & $\mathrm{CH}_{3} \mathrm{CHF}_{2}$ & $\rightarrow$ & $\mathrm{CH}_{3} \mathrm{CHFC}(=\mathrm{O}) \mathrm{OH}$ & $\mathrm{CH}_{3} \mathrm{CF}_{2} \bullet$ & -89.88 & -96.13 & -88.02 & -93.47 & -90.78 & -88.02 \\
\hline $\mathrm{CH}_{3} \mathrm{CHFC}(=\mathrm{O}) \mathrm{O} \bullet$ & + & $\mathrm{CH}_{3} \mathrm{CH}_{2} \mathrm{~F}$ & $\rightarrow$ & $\mathrm{CH}_{3} \mathrm{CHFC}(=\mathrm{O}) \mathrm{OH}$ & $\mathrm{CH}_{2} \mathrm{FCH}_{2} \bullet$ & -89.96 & -96.53 & -89.92 & -93.60 & -92.15 & -93.13 \\
\hline $\mathrm{CH}_{3} \mathrm{CHFC}(=\mathrm{O}) \mathrm{O} \bullet$ & + & $\mathrm{CH}_{3} \mathrm{CHF}_{2}$ & $\rightarrow$ & $\mathrm{CH}_{3} \mathrm{CHFC}(=\mathrm{O}) \mathrm{OH}$ & $\mathrm{CHF}_{2} \mathrm{CH}_{2} \bullet$ & -90.17 & -96.62 & -90.15 & -93.82 & -91.70 & -93.00 \\
\hline $\mathrm{CH}_{3} \mathrm{CHFC}(=\mathrm{O}) \mathrm{O} \bullet$ & + & $\mathrm{CH}_{2} \mathrm{FCH}_{2} \mathrm{~F}$ & $\rightarrow$ & $\mathrm{CH}_{3} \mathrm{CHFC}(=\mathrm{O}) \mathrm{OH}$ & $\mathrm{CH}_{2} \mathrm{FCHF} \bullet$ & -89.57 & -95.63 & -87.25 & -93.27 & -90.96 & -92.82 \\
\hline \multirow[t]{3}{*}{$\mathrm{CH}_{3} \mathrm{CHFC}(=\mathrm{O}) \mathrm{O} \bullet$} & + & $\mathrm{CH}_{3} \mathrm{CF}_{3}$ & $\rightarrow$ & $\mathrm{CH}_{3} \mathrm{CHFC}(=\mathrm{O}) \mathrm{OH}$ & $+\mathrm{CF}_{3} \mathrm{CH}_{2} \bullet$ & -91.58 & -98.00 & -91.45 & -95.33 & -93.40 & -94.15 \\
\hline & & & & & Average & -90.24 & -96.63 & -89.30 & -93.80 & -91.69 & -92.62 \\
\hline & & & & & Uncertainty & 1.12 & 1.50 & 1.14 & 1.17 & 1.06 & 1.11 \\
\hline & & $\mathrm{CH}_{3} \mathrm{CF}_{2} \mathrm{C}($ & & & & & & & & & \\
\hline $\mathrm{CH}_{3} \mathrm{CF}_{2} \mathrm{C}(=\mathrm{O}) \mathrm{O} \bullet$ & + & $\mathrm{CH}_{3} \mathrm{CH}_{3}$ & $\rightarrow$ & $\mathrm{CH}_{3} \mathrm{CF}_{2} \mathrm{C}(=\mathrm{O}) \mathrm{OH}$ & $\mathrm{CH}_{3} \mathrm{CH}_{2} \bullet$ & -141.30 & -147.88 & -140.90 & -144.42 & -142.29 & -145.05 \\
\hline $\mathrm{CH}_{3} \mathrm{CF}_{2} \mathrm{C}(=\mathrm{O}) \mathrm{O} \bullet$ & + & $\mathrm{CH}_{3} \mathrm{CH}_{2} \mathrm{~F}$ & $\rightarrow$ & $\mathrm{CH}_{3} \mathrm{CF}_{2} \mathrm{C}(=\mathrm{O}) \mathrm{OH}$ & $+\mathrm{CH}_{3} \mathrm{CHF} \bullet$ & -140.70 & -147.26 & -139.14 & -144.07 & -141.65 & -144.59 \\
\hline $\mathrm{CH}_{3} \mathrm{CF}_{2} \mathrm{C}(=\mathrm{O}) \mathrm{O} \bullet$ & + & $\mathrm{CH}_{3} \mathrm{CHF}_{2}$ & $\rightarrow$ & $\mathrm{CH}_{3} \mathrm{CF}_{2} \mathrm{C}(=\mathrm{O}) \mathrm{OH}$ & $\mathrm{CH}_{3} \mathrm{CF}_{2} \bullet$ & -140.62 & -146.96 & -138.88 & -144.16 & -141.34 & -144.25 \\
\hline $\mathrm{CH}_{3} \mathrm{CF}_{2} \mathrm{C}(=\mathrm{O}) \mathrm{O} \cdot$ & + & $\mathrm{CH}_{3} \mathrm{CH}_{2} \mathrm{~F}$ & $\rightarrow$ & $\mathrm{CH}_{3} \mathrm{CF}_{2} \mathrm{C}(=\mathrm{O}) \mathrm{OH}$ & $\mathrm{CH}_{2} \mathrm{FCH}_{2} \bullet$ & -140.69 & -147.36 & -140.78 & -144.29 & -142.70 & -144.33 \\
\hline $\mathrm{CH}_{3} \mathrm{CF}_{2} \mathrm{C}(=\mathrm{O}) \mathrm{O} \bullet$ & + & $\mathrm{CH}_{3} \mathrm{CHF}_{2}$ & $\rightarrow$ & $\mathrm{CH}_{3} \mathrm{CF}_{2} \mathrm{C}(=\mathrm{O}) \mathrm{OH}$ & $\mathrm{CHF}_{2} \mathrm{CH}_{2} \bullet$ & -140.91 & -147.44 & -141.01 & -144.51 & -142.26 & -144.20 \\
\hline $\mathrm{CH}_{3} \mathrm{CF}_{2} \mathrm{C}(=\mathrm{O}) \mathrm{O} \bullet$ & + & $\mathrm{CH}_{2} \mathrm{FCH}_{2} \mathrm{~F}$ & $\rightarrow$ & $\mathrm{CH}_{3} \mathrm{CF}_{2} \mathrm{C}(=\mathrm{O}) \mathrm{OH}$ & $\mathrm{CH}_{2} \mathrm{FCHF} \bullet$ & -140.30 & -146.46 & -138.11 & -143.96 & -141.52 & -144.02 \\
\hline \multirow[t]{3}{*}{$\mathrm{CH}_{3} \mathrm{CF}_{2} \mathrm{C}(=\mathrm{O}) \mathrm{O} \bullet$} & + & $\mathrm{CH}_{3} \mathrm{CF}_{3}$ & $\rightarrow$ & $\mathrm{CH}_{3} \mathrm{CF}_{2} \mathrm{C}(=\mathrm{O}) \mathrm{OH}$ & $\mathrm{CF}_{3} \mathrm{CH}_{2} \bullet$ & -142.32 & -148.82 & -142.31 & -146.02 & -143.96 & -145.35 \\
\hline & & & & & Average & -140.98 & -147.45 & -140.16 & -144.49 & -142.24 & -144.54 \\
\hline & & & & & Uncertainty & 0.98 & 1.40 & 1.01 & 1.03 & 0.92 & 0.97 \\
\hline
\end{tabular}




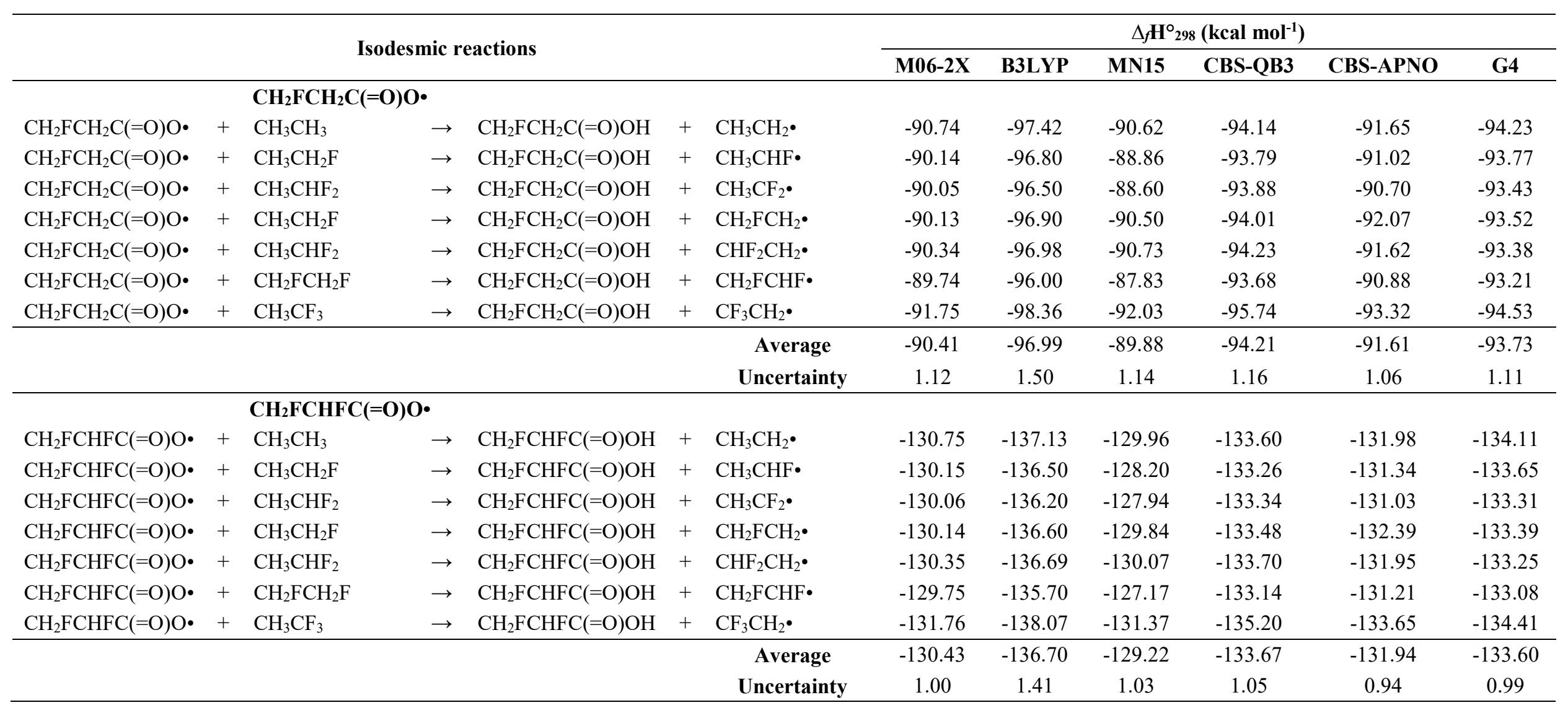




\begin{tabular}{|c|c|c|c|c|c|c|c|c|c|c|c|c|}
\hline \multirow{2}{*}{\multicolumn{7}{|c|}{ Isodesmic reactions }} & \multicolumn{6}{|c|}{$\Delta f H^{\circ}{ }_{298}\left(\mathrm{kcal} \mathrm{mol}^{-1}\right)$} \\
\hline & & & & & & & \multirow[t]{2}{*}{ M06-2X } & \multirow[t]{2}{*}{ B3LYP } & \multirow[t]{2}{*}{ MN15 } & \multirow[t]{2}{*}{ CBS-QB3 } & \multirow[t]{2}{*}{ CBS-APNO } & \multirow[t]{2}{*}{ G4 } \\
\hline & & $\mathrm{CH}_{2} \mathrm{FCF}_{2} \mathrm{CC}$ & & & & & & & & & & \\
\hline $\mathrm{CH}_{2} \mathrm{FCF}_{2} \mathrm{C}(=\mathrm{O}) \mathrm{O} \bullet$ & + & $\mathrm{CH}_{3} \mathrm{CH}_{3}$ & $\rightarrow$ & $\mathrm{CH}_{2} \mathrm{FCF}_{2} \mathrm{C}(=\mathrm{O}) \mathrm{OH}$ & + & $\mathrm{CH}_{3} \mathrm{CH}_{2} \bullet$ & -179.06 & -185.20 & -178.75 & -182.08 & -180.25 & -182.89 \\
\hline $\mathrm{CH}_{2} \mathrm{FCF}_{2} \mathrm{C}(=\mathrm{O}) \mathrm{O} \bullet$ & + & $\mathrm{CH}_{3} \mathrm{CH}_{2} \mathrm{~F}$ & $\rightarrow$ & $\mathrm{CH}_{2} \mathrm{FCF}_{2} \mathrm{C}(=\mathrm{O}) \mathrm{OH}$ & + & $\mathrm{CH}_{3} \mathrm{CHF} \bullet$ & -178.46 & -184.57 & -176.99 & -181.73 & -179.62 & -182.43 \\
\hline $\mathrm{CH}_{2} \mathrm{FCF}_{2} \mathrm{C}(=\mathrm{O}) \mathrm{O} \bullet$ & + & $\mathrm{CH}_{3} \mathrm{CHF}_{2}$ & $\rightarrow$ & $\mathrm{CH}_{2} \mathrm{FCF}_{2} \mathrm{C}(=\mathrm{O}) \mathrm{OH}$ & + & $\mathrm{CH}_{3} \mathrm{CF}_{2} \bullet$ & -178.37 & -184.27 & -176.74 & -181.82 & -179.30 & -182.09 \\
\hline $\mathrm{CH}_{2} \mathrm{FCF}_{2} \mathrm{C}(=\mathrm{O}) \mathrm{O} \bullet$ & + & $\mathrm{CH}_{3} \mathrm{CH}_{2} \mathrm{~F}$ & $\rightarrow$ & $\mathrm{CH}_{2} \mathrm{FCF}_{2} \mathrm{C}(=\mathrm{O}) \mathrm{OH}$ & + & $\mathrm{CH}_{2} \mathrm{FCH}_{2} \bullet$ & -178.45 & -184.68 & -178.63 & -181.95 & -180.67 & -182.18 \\
\hline $\mathrm{CH}_{2} \mathrm{FCF}_{2} \mathrm{C}(=\mathrm{O}) \mathrm{O} \bullet$ & + & $\mathrm{CH}_{3} \mathrm{CHF}_{2}$ & $\rightarrow$ & $\mathrm{CH}_{2} \mathrm{FCF}_{2} \mathrm{C}(=\mathrm{O}) \mathrm{OH}$ & + & $\mathrm{CHF}_{2} \mathrm{CH}_{2} \bullet$ & -178.66 & -184.76 & -178.86 & -182.17 & -180.22 & -182.04 \\
\hline $\mathrm{CH}_{2} \mathrm{FCF}_{2} \mathrm{C}(=\mathrm{O}) \mathrm{O} \bullet$ & + & $\mathrm{CH}_{2} \mathrm{FCH}_{2} \mathrm{~F}$ & $\rightarrow$ & $\mathrm{CH}_{2} \mathrm{FCF}_{2} \mathrm{C}(=\mathrm{O}) \mathrm{OH}$ & + & $\mathrm{CH}_{2} \mathrm{FCHF} \bullet$ & -178.06 & -183.77 & -175.97 & -181.62 & -179.48 & -181.86 \\
\hline \multirow[t]{4}{*}{$\mathrm{CH}_{2} \mathrm{FCF}_{2} \mathrm{C}(=\mathrm{O}) \mathrm{O} \bullet$} & + & $\mathrm{CH}_{3} \mathrm{CF}_{3}$ & $\rightarrow$ & $\mathrm{CH}_{2} \mathrm{FCF}_{2} \mathrm{C}(=\mathrm{O}) \mathrm{OH}$ & + & $\mathrm{CF}_{3} \mathrm{CH}_{2} \bullet$ & -180.07 & -186.14 & -180.17 & -183.68 & -181.92 & -183.19 \\
\hline & & & & & & Average & -178.74 & -184.77 & -178.02 & -182.15 & -180.21 & -182.38 \\
\hline & & & & & & Uncertainty & 1.06 & 1.45 & 1.08 & 1.11 & 1.00 & 1.05 \\
\hline & & $\mathrm{CHF}_{2} \mathrm{CH}_{2} \mathrm{C}$ & & & & & & & & & & \\
\hline $\mathrm{CHF}_{2} \mathrm{CH}_{2} \mathrm{C}(=\mathrm{O}) \mathrm{O} \bullet$ & + & $\mathrm{CH}_{3} \mathrm{CH}_{3}$ & $\rightarrow$ & $\mathrm{CHF}_{2} \mathrm{CH}_{2} \mathrm{C}(=\mathrm{O}) \mathrm{OH}$ & + & $\mathrm{CH}_{3} \mathrm{CH}_{2} \bullet$ & -143.50 & -150.06 & -143.13 & -146.78 & -144.58 & -143.13 \\
\hline $\mathrm{CHF}_{2} \mathrm{CH}_{2} \mathrm{C}(=\mathrm{O}) \mathrm{O} \bullet$ & + & $\mathrm{CH}_{3} \mathrm{CH}_{2} \mathrm{~F}$ & $\rightarrow$ & $\mathrm{CHF}_{2} \mathrm{CH}_{2} \mathrm{C}(=\mathrm{O}) \mathrm{OH}$ & + & $\mathrm{CH}_{3} \mathrm{CHF} \bullet$ & -142.90 & -149.43 & -141.37 & -146.43 & -143.94 & -146.85 \\
\hline $\mathrm{CHF}_{2} \mathrm{CH}_{2} \mathrm{C}(=\mathrm{O}) \mathrm{O} \bullet$ & + & $\mathrm{CH}_{3} \mathrm{CHF}_{2}$ & $\rightarrow$ & $\mathrm{CHF}_{2} \mathrm{CH}_{2} \mathrm{C}(=\mathrm{O}) \mathrm{OH}$ & + & $\mathrm{CH}_{3} \mathrm{CF}_{2} \bullet$ & -142.81 & -149.13 & -141.11 & -146.52 & -143.63 & -146.52 \\
\hline $\mathrm{CHF}_{2} \mathrm{CH}_{2} \mathrm{C}(=\mathrm{O}) \mathrm{O} \bullet$ & + & $\mathrm{CH}_{3} \mathrm{CH}_{2} \mathrm{~F}$ & $\rightarrow$ & $\mathrm{CHF}_{2} \mathrm{CH}_{2} \mathrm{C}(=\mathrm{O}) \mathrm{OH}$ & + & $\mathrm{CH}_{2} \mathrm{FCH}_{2} \bullet$ & -142.89 & -149.53 & -143.01 & -146.65 & -144.99 & -146.60 \\
\hline $\mathrm{CHF}_{2} \mathrm{CH}_{2} \mathrm{C}(=\mathrm{O}) \mathrm{O} \bullet$ & + & $\mathrm{CH}_{3} \mathrm{CHF}_{2}$ & $\rightarrow$ & $\mathrm{CHF}_{2} \mathrm{CH}_{2} \mathrm{C}(=\mathrm{O}) \mathrm{OH}$ & + & $\mathrm{CHF}_{2} \mathrm{CH}_{2} \bullet$ & -143.10 & -149.62 & -143.24 & -146.87 & -144.55 & -146.46 \\
\hline $\mathrm{CHF}_{2} \mathrm{CH}_{2} \mathrm{C}(=\mathrm{O}) \mathrm{O} \bullet$ & + & $\mathrm{CH}_{2} \mathrm{FCH}_{2} \mathrm{~F}$ & $\rightarrow$ & $\mathrm{CHF}_{2} \mathrm{CH}_{2} \mathrm{C}(=\mathrm{O}) \mathrm{OH}$ & + & $\mathrm{CH}_{2} \mathrm{FCHF} \bullet$ & -142.50 & -148.63 & -140.34 & -146.32 & -143.81 & -146.29 \\
\hline \multirow[t]{3}{*}{$\mathrm{CHF}_{2} \mathrm{CH}_{2} \mathrm{C}(=\mathrm{O}) \mathrm{O} \bullet$} & + & $\mathrm{CH}_{3} \mathrm{CF}_{3}$ & $\rightarrow$ & $\mathrm{CHF}_{2} \mathrm{CH}_{2} \mathrm{C}(=\mathrm{O}) \mathrm{OH}$ & + & $\mathrm{CF}_{3} \mathrm{CH}_{2} \bullet$ & -144.51 & -151.00 & -144.54 & -148.38 & -146.25 & -147.62 \\
\hline & & & & & & Average & -143.17 & -149.63 & -142.39 & -146.85 & -144.54 & -146.21 \\
\hline & & & & & & Uncertainty & 1.00 & 1.41 & 1.03 & 1.05 & 0.94 & 0.99 \\
\hline
\end{tabular}




\begin{tabular}{|c|c|c|c|c|c|c|c|c|c|c|c|}
\hline \multirow{2}{*}{\multicolumn{6}{|c|}{ Isodesmic reactions }} & \multicolumn{6}{|c|}{$\Delta_{f} \mathbf{H}^{\circ}{ }_{298}\left(\mathrm{kcal} \mathrm{mol}^{-1}\right)$} \\
\hline & & & & & & \multirow[t]{2}{*}{ M06-2X } & \multirow[t]{2}{*}{ B3LYP } & \multirow[t]{2}{*}{ MN15 } & \multirow[t]{2}{*}{ CBS-QB3 } & \multirow[t]{2}{*}{ CBS-APNO } & \multirow[t]{2}{*}{ G4 } \\
\hline & & $\mathrm{CHF}_{2} \mathrm{CHFC}$ & & & & & & & & & \\
\hline $\mathrm{CHF}_{2} \mathrm{CHFC}(=\mathrm{O}) \mathrm{O} \bullet$ & + & $\mathrm{CH}_{3} \mathrm{CH}_{3}$ & $\rightarrow$ & $\mathrm{CHF}_{2} \mathrm{CHFC}(=\mathrm{O}) \mathrm{OH}$ & $+\mathrm{CH}_{3} \mathrm{CH}_{2} \bullet$ & -178.79 & -185.89 & -179.84 & -182.82 & -181.09 & -183.56 \\
\hline $\mathrm{CHF}_{2} \mathrm{CHFC}(=\mathrm{O}) \mathrm{O} \bullet$ & + & $\mathrm{CH}_{3} \mathrm{CH}_{2} \mathrm{~F}$ & $\rightarrow$ & $\mathrm{CHF}_{2} \mathrm{CHFC}(=\mathrm{O}) \mathrm{OH}$ & $+\mathrm{CH}_{3} \mathrm{CHF} \bullet$ & -178.19 & -185.26 & -178.08 & -182.47 & -180.46 & -183.11 \\
\hline $\mathrm{CHF}_{2} \mathrm{CHFC}(=\mathrm{O}) \mathrm{O} \bullet$ & + & $\mathrm{CH}_{3} \mathrm{CHF}_{2}$ & $\rightarrow$ & $\mathrm{CHF}_{2} \mathrm{CHFC}(=\mathrm{O}) \mathrm{OH}$ & $+\mathrm{CH}_{3} \mathrm{CF}_{2} \bullet$ & -178.10 & -184.96 & -177.82 & -182.56 & -180.14 & -182.77 \\
\hline $\mathrm{CHF}_{2} \mathrm{CHFC}(=\mathrm{O}) \mathrm{O} \bullet$ & + & $\mathrm{CH}_{3} \mathrm{CH}_{2} \mathrm{~F}$ & $\rightarrow$ & $\mathrm{CHF}_{2} \mathrm{CHFC}(=\mathrm{O}) \mathrm{OH}$ & $+\mathrm{CH}_{2} \mathrm{FCH}_{2} \bullet$ & -178.18 & -185.37 & -179.72 & -182.69 & -181.51 & -182.85 \\
\hline $\mathrm{CHF}_{2} \mathrm{CHFC}(=\mathrm{O}) \mathrm{O} \bullet$ & + & $\mathrm{CH}_{3} \mathrm{CHF}_{2}$ & $\rightarrow$ & $\mathrm{CHF}_{2} \mathrm{CHFC}(=\mathrm{O}) \mathrm{OH}$ & $+\mathrm{CHF}_{2} \mathrm{CH}_{2} \bullet$ & -178.39 & -185.45 & -179.94 & -182.91 & -181.06 & -182.71 \\
\hline $\mathrm{CHF}_{2} \mathrm{CHFC}(=\mathrm{O}) \mathrm{O} \bullet$ & + & $\mathrm{CH}_{2} \mathrm{FCH}_{2} \mathrm{~F}$ & $\rightarrow$ & $\mathrm{CHF}_{2} \mathrm{CHFC}(=\mathrm{O}) \mathrm{OH}$ & $+\mathrm{CH}_{2} \mathrm{FCHF} \bullet$ & -177.79 & -184.46 & -177.05 & -182.36 & -180.32 & -182.54 \\
\hline \multirow[t]{4}{*}{$\mathrm{CHF}_{2} \mathrm{CHFC}(=\mathrm{O}) \mathrm{O} \bullet$} & + & $\mathrm{CH}_{3} \mathrm{CF}_{3}$ & $\rightarrow$ & $\mathrm{CHF}_{2} \mathrm{CHFC}(=\mathrm{O}) \mathrm{OH}$ & $+\mathrm{CF}_{3} \mathrm{CH}_{2} \bullet$ & -179.80 & -186.83 & -181.25 & -184.42 & -182.76 & -183.87 \\
\hline & & & & & Average & -178.46 & -185.46 & -179.10 & -182.89 & -181.05 & -183.06 \\
\hline & & & & & Uncertainty & 1.06 & 1.45 & 1.08 & 1.11 & 1.00 & 1.05 \\
\hline & & $\mathrm{CHF}_{2} \mathrm{CF}_{2} \mathrm{C}$ & & & & & & & & & \\
\hline $\mathrm{CHF}_{2} \mathrm{CF}_{2} \mathrm{C}(=\mathrm{O}) \mathrm{O} \bullet$ & + & $\mathrm{CH}_{3} \mathrm{CH}_{3}$ & $\rightarrow$ & $\mathrm{CHF}_{2} \mathrm{CF}_{2} \mathrm{C}(=\mathrm{O}) \mathrm{OH}$ & $+\mathrm{CH}_{3} \mathrm{CH}_{2} \bullet$ & -227.96 & -234.26 & -227.39 & -231.29 & -228.87 & -232.29 \\
\hline $\mathrm{CHF}_{2} \mathrm{CF}_{2} \mathrm{C}(=\mathrm{O}) \mathrm{O} \bullet$ & + & $\mathrm{CH}_{3} \mathrm{CH}_{2} \mathrm{~F}$ & $\rightarrow$ & $\mathrm{CHF}_{2} \mathrm{CF}_{2} \mathrm{C}(=\mathrm{O}) \mathrm{OH}$ & $+\mathrm{CH}_{3} \mathrm{CHF} \bullet$ & -227.36 & -233.63 & -225.63 & -230.94 & -228.23 & -231.83 \\
\hline $\mathrm{CHF}_{2} \mathrm{CF}_{2} \mathrm{C}(=\mathrm{O}) \mathrm{O} \bullet$ & + & $\mathrm{CH}_{3} \mathrm{CHF}_{2}$ & $\rightarrow$ & $\mathrm{CHF}_{2} \mathrm{CF}_{2} \mathrm{C}(=\mathrm{O}) \mathrm{OH}$ & $+\mathrm{CH}_{3} \mathrm{CF}_{2} \bullet$ & -227.27 & -233.33 & -225.37 & -231.03 & -227.92 & -231.50 \\
\hline $\mathrm{CHF}_{2} \mathrm{CF}_{2} \mathrm{C}(=\mathrm{O}) \mathrm{O} \cdot$ & + & $\mathrm{CH}_{3} \mathrm{CH}_{2} \mathrm{~F}$ & $\rightarrow$ & $\mathrm{CHF}_{2} \mathrm{CF}_{2} \mathrm{C}(=\mathrm{O}) \mathrm{OH}$ & $+\mathrm{CH}_{2} \mathrm{FCH}_{2} \bullet$ & -227.35 & -233.73 & -227.27 & -231.16 & -229.28 & -231.58 \\
\hline $\mathrm{CHF}_{2} \mathrm{CF}_{2} \mathrm{C}(=\mathrm{O}) \mathrm{O} \bullet$ & + & $\mathrm{CH}_{3} \mathrm{CHF}_{2}$ & $\rightarrow$ & $\mathrm{CHF}_{2} \mathrm{CF}_{2} \mathrm{C}(=\mathrm{O}) \mathrm{OH}$ & $+\mathrm{CHF}_{2} \mathrm{CH}_{2} \bullet$ & -227.56 & -233.81 & -227.50 & -231.38 & -228.84 & -231.44 \\
\hline $\mathrm{CHF}_{2} \mathrm{CF}_{2} \mathrm{C}(=\mathrm{O}) \mathrm{O} \bullet$ & + & $\mathrm{CH}_{2} \mathrm{FCH}_{2} \mathrm{~F}$ & $\rightarrow$ & $\mathrm{CHF}_{2} \mathrm{CF}_{2} \mathrm{C}(=\mathrm{O}) \mathrm{OH}$ & $+\mathrm{CH}_{2} \mathrm{FCHF} \bullet$ & -226.96 & -232.83 & -224.60 & -230.83 & -228.10 & -231.27 \\
\hline \multirow[t]{3}{*}{$\mathrm{CHF}_{2} \mathrm{CF}_{2} \mathrm{C}(=\mathrm{O}) \mathrm{O} \bullet$} & + & $\mathrm{CH}_{3} \mathrm{CF}_{3}$ & $\rightarrow$ & $\mathrm{CHF}_{2} \mathrm{CF}_{2} \mathrm{C}(=\mathrm{O}) \mathrm{OH}$ & $+\mathrm{CF}_{3} \mathrm{CH}_{2} \bullet$ & -228.97 & -235.19 & -228.80 & -232.89 & -230.54 & -232.60 \\
\hline & & & & & Average & -227.63 & -233.83 & -226.65 & -231.36 & -228.83 & -231.79 \\
\hline & & & & & Uncertainty & 1.19 & 1.55 & 1.20 & 1.23 & 1.13 & 1.17 \\
\hline
\end{tabular}




\begin{tabular}{|c|c|c|c|c|c|c|c|c|c|c|c|c|}
\hline \multirow{2}{*}{\multicolumn{7}{|c|}{ Isodesmic reactions }} & \multicolumn{6}{|c|}{$\Delta_{f} \mathrm{H}^{\circ} 298\left(\mathrm{kcal} \mathrm{mol}^{-1}\right)$} \\
\hline & & & & & & & \multirow[t]{2}{*}{ M06-2X } & \multirow[t]{2}{*}{ B3LYP } & \multirow[t]{2}{*}{ MN15 } & \multirow[t]{2}{*}{ CBS-QB3 } & \multirow[t]{2}{*}{ CBS-APNO } & \multirow[t]{2}{*}{ G4 } \\
\hline & & $\mathrm{CF}_{3} \mathrm{CH}_{2} \mathrm{CC}$ & & & & & & & & & & \\
\hline $\mathrm{CF}_{3} \mathrm{CH}_{2} \mathrm{C}(=\mathrm{O}) \mathrm{O} \bullet$ & + & $\mathrm{CH}_{3} \mathrm{CH}_{3}$ & $\rightarrow$ & $\mathrm{CF}_{3} \mathrm{CH}_{2} \mathrm{C}(=\mathrm{O}) \mathrm{OH}$ & + & $\mathrm{CH}_{3} \mathrm{CH}_{2} \bullet$ & -199.60 & -206.87 & -200.25 & -203.51 & -200.56 & -204.30 \\
\hline $\mathrm{CF}_{3} \mathrm{CH}_{2} \mathrm{C}(=\mathrm{O}) \mathrm{O} \bullet$ & + & $\mathrm{CH}_{3} \mathrm{CH}_{2} \mathrm{~F}$ & $\rightarrow$ & $\mathrm{CF}_{3} \mathrm{CH}_{2} \mathrm{C}(=\mathrm{O}) \mathrm{OH}$ & + & $\mathrm{CH}_{3} \mathrm{CHF} \bullet$ & -199.00 & -206.24 & -198.49 & -203.17 & -199.92 & -203.84 \\
\hline $\mathrm{CF}_{3} \mathrm{CH}_{2} \mathrm{C}(=\mathrm{O}) \mathrm{O} \bullet$ & + & $\mathrm{CH}_{3} \mathrm{CHF}_{2}$ & $\rightarrow$ & $\mathrm{CF}_{3} \mathrm{CH}_{2} \mathrm{C}(=\mathrm{O}) \mathrm{OH}$ & + & $\mathrm{CH}_{3} \mathrm{CF}_{2} \bullet$ & -198.91 & -205.94 & -198.23 & -203.25 & -199.60 & -203.50 \\
\hline $\mathrm{CF}_{3} \mathrm{CH}_{2} \mathrm{C}(=\mathrm{O}) \mathrm{O} \bullet$ & + & $\mathrm{CH}_{3} \mathrm{CH}_{2} \mathrm{~F}$ & $\rightarrow$ & $\mathrm{CF}_{3} \mathrm{CH}_{2} \mathrm{C}(=\mathrm{O}) \mathrm{OH}$ & + & $\mathrm{CH}_{2} \mathrm{FCH}_{2} \bullet$ & -198.99 & -206.34 & -200.13 & -203.39 & -200.97 & -203.59 \\
\hline $\mathrm{CF}_{3} \mathrm{CH}_{2} \mathrm{C}(=\mathrm{O}) \mathrm{O} \bullet$ & + & $\mathrm{CH}_{3} \mathrm{CHF}_{2}$ & $\rightarrow$ & $\mathrm{CF}_{3} \mathrm{CH}_{2} \mathrm{C}(=\mathrm{O}) \mathrm{OH}$ & + & $\mathrm{CHF}_{2} \mathrm{CH}_{2} \bullet$ & -206.43 & -203.60 & -200.36 & -200.53 & -200.53 & -203.45 \\
\hline $\mathrm{CF}_{3} \mathrm{CH}_{2} \mathrm{C}(=\mathrm{O}) \mathrm{O} \bullet$ & + & $\mathrm{CH}_{2} \mathrm{FCH}_{2} \mathrm{~F}$ & $\rightarrow$ & $\mathrm{CF}_{3} \mathrm{CH}_{2} \mathrm{C}(=\mathrm{O}) \mathrm{OH}$ & + & $\mathrm{CH}_{2} \mathrm{FCHF} \bullet$ & -205.44 & -203.05 & -197.46 & -199.79 & -199.79 & -203.27 \\
\hline \multirow[t]{4}{*}{$\mathrm{CF}_{3} \mathrm{CH}_{2} \mathrm{C}(=\mathrm{O}) \mathrm{O} \bullet$} & + & $\mathrm{CH}_{3} \mathrm{CF}_{3}$ & $\rightarrow$ & $\mathrm{CF}_{3} \mathrm{CH}_{2} \mathrm{C}(=\mathrm{O}) \mathrm{OH}$ & + & $\mathrm{CF}_{3} \mathrm{CH}_{2} \bullet$ & -200.61 & -205.11 & -201.66 & -202.22 & -202.22 & -204.60 \\
\hline & & & & & & Average & -201.28 & -205.31 & -199.51 & -202.26 & -200.51 & -203.79 \\
\hline & & & & & & Uncertainty & 1.05 & 1.45 & 1.07 & 1.10 & 0.99 & 1.04 \\
\hline & & $\mathrm{CF}_{3} \mathrm{CHFC}$ & & & & & & & & & & \\
\hline $\mathrm{CF}_{3} \mathrm{CHFC}(=\mathrm{O}) \mathrm{O} \bullet$ & + & $\mathrm{CH}_{3} \mathrm{CH}_{3}$ & $\rightarrow$ & $\mathrm{CF}_{3} \mathrm{CHFC}(=\mathrm{O}) \mathrm{OH}$ & + & $\mathrm{CH}_{3} \mathrm{CH}_{2} \bullet$ & -235.72 & -242.79 & -235.91 & -239.56 & -237.27 & -240.65 \\
\hline $\mathrm{CF}_{3} \mathrm{CHFC}(=\mathrm{O}) \mathrm{O} \bullet$ & + & $\mathrm{CH}_{3} \mathrm{CH}_{2} \mathrm{~F}$ & $\rightarrow$ & $\mathrm{CF}_{3} \mathrm{CHFC}(=\mathrm{O}) \mathrm{OH}$ & + & $\mathrm{CH}_{3} \mathrm{CHF} \bullet$ & -235.12 & -242.16 & -234.15 & -239.21 & -236.63 & -240.19 \\
\hline $\mathrm{CF}_{3} \mathrm{CHFC}(=\mathrm{O}) \mathrm{O} \bullet$ & + & $\mathrm{CH}_{3} \mathrm{CHF}_{2}$ & $\rightarrow$ & $\mathrm{CF}_{3} \mathrm{CHFC}(=\mathrm{O}) \mathrm{OH}$ & + & $\mathrm{CH}_{3} \mathrm{CF}_{2} \bullet$ & -235.03 & -241.86 & -233.89 & -239.30 & -236.31 & -239.86 \\
\hline $\mathrm{CF}_{3} \mathrm{CHFC}(=\mathrm{O}) \mathrm{O} \bullet$ & + & $\mathrm{CH}_{3} \mathrm{CH}_{2} \mathrm{~F}$ & $\rightarrow$ & $\mathrm{CF}_{3} \mathrm{CHFC}(=\mathrm{O}) \mathrm{OH}$ & + & $\mathrm{CH}_{2} \mathrm{FCH}_{2} \bullet$ & -235.11 & -242.26 & -235.79 & -239.43 & -237.68 & -239.94 \\
\hline $\mathrm{CF}_{3} \mathrm{CHFC}(=\mathrm{O}) \mathrm{O} \bullet$ & + & $\mathrm{CH}_{3} \mathrm{CHF}_{2}$ & $\rightarrow$ & $\mathrm{CF}_{3} \mathrm{CHFC}(=\mathrm{O}) \mathrm{OH}$ & + & $\mathrm{CHF}_{2} \mathrm{CH}_{2} \bullet$ & -235.32 & -242.34 & -236.01 & -239.65 & -237.24 & -239.80 \\
\hline $\mathrm{CF}_{3} \mathrm{CHFC}(=\mathrm{O}) \mathrm{O} \bullet$ & + & $\mathrm{CH}_{2} \mathrm{FCH}_{2} \mathrm{~F}$ & $\rightarrow$ & $\mathrm{CF}_{3} \mathrm{CHFC}(=\mathrm{O}) \mathrm{OH}$ & + & $\mathrm{CH}_{2} \mathrm{FCHF} \bullet$ & -234.72 & -241.36 & -233.12 & -239.10 & -236.50 & -239.63 \\
\hline \multirow[t]{3}{*}{$\mathrm{CF}_{3} \mathrm{CHFC}(=\mathrm{O}) \mathrm{O} \bullet$} & + & $\mathrm{CH}_{3} \mathrm{CF}_{3}$ & $\rightarrow$ & $\mathrm{CF}_{3} \mathrm{CHFC}(=\mathrm{O}) \mathrm{OH}$ & + & $\mathrm{CF}_{3} \mathrm{CH}_{2} \bullet$ & -236.73 & -243.72 & -237.32 & -241.16 & -238.93 & -240.96 \\
\hline & & & & & & Average & -235.39 & -242.36 & -235.17 & -239.63 & -237.22 & -240.15 \\
\hline & & & & & & Uncertainty & 1.20 & 1.56 & 1.21 & 1.24 & 1.14 & 1.18 \\
\hline
\end{tabular}




\begin{tabular}{|c|c|c|c|c|c|c|c|c|c|c|c|}
\hline \multirow{2}{*}{\multicolumn{6}{|c|}{ Isodesmic reactions }} & \multicolumn{6}{|c|}{$\Delta_{f} \mathrm{H}^{\circ}{ }_{298}\left(\mathrm{kcal} \mathrm{mol}^{-1}\right)$} \\
\hline & & & & & & \multirow[t]{2}{*}{ M06-2X } & \multirow[t]{2}{*}{ B3LYP } & \multirow[t]{2}{*}{ MN15 } & \multirow[t]{2}{*}{ CBS-QB3 } & \multirow[t]{2}{*}{ CBS-APNO } & \multirow[t]{2}{*}{ G4 } \\
\hline & & $\mathrm{CF}_{3} \mathrm{CF}_{2} \mathrm{C}$ & & & & & & & & & \\
\hline $\mathrm{CF}_{3} \mathrm{CF}_{2} \mathrm{C}(=\mathrm{O}) \mathrm{O} \bullet$ & + & $\mathrm{CH}_{3} \mathrm{CH}_{3}$ & $\rightarrow$ & $\mathrm{CF}_{3} \mathrm{CF}_{2} \mathrm{C}(=\mathrm{O}) \mathrm{OH}$ & $+\mathrm{CH}_{3} \mathrm{CH}_{2} \bullet$ & -281.57 & -288.70 & -281.34 & -286.10 & -282.98 & -287.62 \\
\hline $\mathrm{CF}_{3} \mathrm{CF}_{2} \mathrm{C}(=\mathrm{O}) \mathrm{O} \bullet$ & + & $\mathrm{CH}_{3} \mathrm{CH}_{2} \mathrm{~F}$ & $\rightarrow$ & $\mathrm{CF}_{3} \mathrm{CF}_{2} \mathrm{C}(=\mathrm{O}) \mathrm{OH}$ & $\mathrm{CH}_{3} \mathrm{CHF} \bullet$ & -280.97 & -288.07 & -279.58 & -285.75 & -282.34 & -287.16 \\
\hline $\mathrm{CF}_{3} \mathrm{CF}_{2} \mathrm{C}(=\mathrm{O}) \mathrm{O} \bullet$ & + & $\mathrm{CH}_{3} \mathrm{CHF}_{2}$ & $\rightarrow$ & $\mathrm{CF}_{3} \mathrm{CF}_{2} \mathrm{C}(=\mathrm{O}) \mathrm{OH}$ & $\mathrm{CH}_{3} \mathrm{CF}_{2} \bullet$ & -280.89 & -287.77 & -279.32 & -285.84 & -282.02 & -286.82 \\
\hline $\mathrm{CF}_{3} \mathrm{CF}_{2} \mathrm{C}(=\mathrm{O}) \mathrm{O} \bullet$ & + & $\mathrm{CH}_{3} \mathrm{CH}_{2} \mathrm{~F}$ & $\rightarrow$ & $\mathrm{CF}_{3} \mathrm{CF}_{2} \mathrm{C}(=\mathrm{O}) \mathrm{OH}$ & $+\mathrm{CH}_{2} \mathrm{FCH}_{2} \bullet$ & -280.96 & -288.17 & -281.22 & -285.97 & -283.39 & -286.91 \\
\hline $\mathrm{CF}_{3} \mathrm{CF}_{2} \mathrm{C}(=\mathrm{O}) \mathrm{O} \bullet$ & + & $\mathrm{CH}_{3} \mathrm{CHF}_{2}$ & $\rightarrow$ & $\mathrm{CF}_{3} \mathrm{CF}_{2} \mathrm{C}(=\mathrm{O}) \mathrm{OH}$ & $+\mathrm{CHF}_{2} \mathrm{CH}_{2} \bullet$ & -281.17 & -288.26 & -281.45 & -286.19 & -282.95 & -286.77 \\
\hline $\mathrm{CF}_{3} \mathrm{CF}_{2} \mathrm{C}(=\mathrm{O}) \mathrm{O} \bullet$ & + & $\mathrm{CH}_{2} \mathrm{FCH}_{2} \mathrm{~F}$ & $\rightarrow$ & $\mathrm{CF}_{3} \mathrm{CF}_{2} \mathrm{C}(=\mathrm{O}) \mathrm{OH}$ & $\mathrm{CH}_{2} \mathrm{FCHF} \bullet$ & -280.57 & -287.27 & -278.55 & -285.64 & -282.21 & -286.59 \\
\hline \multirow[t]{3}{*}{$\mathrm{CF}_{3} \mathrm{CF}_{2} \mathrm{C}(=\mathrm{O}) \mathrm{O} \bullet$} & + & $\mathrm{CH}_{3} \mathrm{CF}_{3}$ & $\rightarrow$ & $\mathrm{CF}_{3} \mathrm{CF}_{2} \mathrm{C}(=\mathrm{O}) \mathrm{OH}$ & $+\mathrm{CF}_{3} \mathrm{CH}_{2} \bullet$ & -282.58 & -289.64 & -282.75 & -287.70 & -284.64 & -287.92 \\
\hline & & & & & Average & -281.25 & -288.27 & -280.60 & -286.17 & -282.93 & -287.11 \\
\hline & & & & & Uncertainty & 1.30 & 1.63 & 1.31 & 1.33 & 1.24 & 1.28 \\
\hline
\end{tabular}




\section{Chemical Compounds}

\begin{tabular}{|c|c|c|c|}
\hline Molecular formulas $^{a}$ & Nomenclature & Molecular formulas $^{a}$ & Nomenclature \\
\hline $\mathrm{CH}_{3} \mathrm{C}(=\mathrm{O}) \mathrm{OH}$ & acetic acid/ethanoic acid & $\mathrm{CHF} \cdot \mathrm{CH}_{2} \mathrm{C}(=\mathrm{O}) \mathrm{OH}$ & carboxy-2-fluoroeth-2yl \\
\hline $\mathrm{CH}_{2} \mathrm{FC}(=\mathrm{O}) \mathrm{OH}$ & 2-fluoroacetic acid & $\mathrm{CHF} \cdot \mathrm{CHFC}(=\mathrm{O}) \mathrm{OH}$ & carboxy-1,2-difluoroeth-2yl \\
\hline $\mathrm{CHF}_{2} \mathrm{C}(=\mathrm{O}) \mathrm{OH}$ & 2,2-difluoroacetic acid & $\mathrm{CHF} \cdot \mathrm{CF}_{2} \mathrm{C}(=\mathrm{O}) \mathrm{OH}$ & carboxy-1,1,2-trifluoroeth-2yl \\
\hline $\mathrm{CF}_{3} \mathrm{C}(=\mathrm{O}) \mathrm{OH}$ & 2,2,2-trifluoroacetic acid & $\mathrm{CF}_{2} \cdot \mathrm{CH}_{2} \mathrm{C}(=\mathrm{O}) \mathrm{OH}$ & carboxy-2,2-difluoroeth-2yl \\
\hline $\mathrm{CH}_{3} \mathrm{CH}_{2} \mathrm{C}(=\mathrm{O}) \mathrm{OH}$ & propionic acid & $\mathrm{CF}_{2} \cdot \mathrm{CHFC}(=\mathrm{O}) \mathrm{OH}$ & carboxy-1,2,2-trifluoroeth-2yl \\
\hline $\mathrm{CH}_{2} \mathrm{FCH}_{2} \mathrm{C}(=\mathrm{O}) \mathrm{OH}$ & 3-fluoropropionic acid & $\mathrm{CF}_{2} \cdot \mathrm{CF}_{2} \mathrm{C}(=\mathrm{O}) \mathrm{OH}$ & carboxy-1,1,2,2-tetrafluoroeth-2yl \\
\hline $\mathrm{CHF}_{2} \mathrm{CH}_{2} \mathrm{C}(=\mathrm{O}) \mathrm{OH}$ & 3,3-difluoropropionic acid & $\mathrm{CH}_{3} \mathrm{CH} \cdot \mathrm{C}(=\mathrm{O}) \mathrm{OH}$ & carboxyeth-1yl \\
\hline $\mathrm{CF}_{3} \mathrm{CH}_{2} \mathrm{C}(=\mathrm{O}) \mathrm{OH}$ & 3,3,3-trifluoropropionic acid & $\mathrm{CH}_{2} \mathrm{FCH} \cdot \mathrm{C}(=\mathrm{O}) \mathrm{OH}$ & carboxy-2-fluoroeth-1yl \\
\hline $\mathrm{CH}_{3} \mathrm{CHFC}(=\mathrm{O}) \mathrm{OH}$ & 2-fluoropropionic acid & $\mathrm{CHF}_{2} \mathrm{CH} \cdot \mathrm{C}(=\mathrm{O}) \mathrm{OH}$ & carboxy-2,2-difluoroeth-1yl \\
\hline $\mathrm{CH}_{3} \mathrm{CF}_{2} \mathrm{C}(=\mathrm{O}) \mathrm{OH}$ & 2,2-difluoropropionic acid & $\mathrm{CF}_{3} \mathrm{CH} \cdot \mathrm{C}(=\mathrm{O}) \mathrm{OH}$ & carboxy-2,2,2-trifluoroeth-1yl \\
\hline $\mathrm{CH}_{2} \mathrm{FCHFC}(=\mathrm{O}) \mathrm{OH}$ & 2,3-difluoropropionic acid & $\mathrm{CH}_{3} \mathrm{CF} \cdot \mathrm{C}(=\mathrm{O}) \mathrm{OH}$ & carboxy-1-fluoroeth-1yl \\
\hline $\mathrm{CH}_{2} \mathrm{FCF}_{2} \mathrm{C}(=\mathrm{O}) \mathrm{OH}$ & 2,2,3-trifluoropropionic acid & $\mathrm{CH}_{2} \mathrm{FCF} \cdot \mathrm{C}(=\mathrm{O}) \mathrm{OH}$ & carboxy-1,2-difluoroeth-1yl \\
\hline $\mathrm{CHF}_{2} \mathrm{CHFC}(=\mathrm{O}) \mathrm{OH}$ & 2,3,3-trifluoropropionic acid & $\mathrm{CHF}_{2} \mathrm{CF} \cdot \mathrm{C}(=\mathrm{O}) \mathrm{OH}$ & carboxy-1,2,2-trifluoroeth-1yl \\
\hline $\mathrm{CHF}_{2} \mathrm{CF}_{2} \mathrm{C}(=\mathrm{O}) \mathrm{OH}$ & 2,2,3,3-tetrafluoropropionic acid & $\mathrm{CF}_{3} \mathrm{CF} \cdot \mathrm{C}(=\mathrm{O}) \mathrm{OH}$ & carboxy-1,2,2,2-tetrafluoroeth-1yl \\
\hline $\mathrm{CF}_{3} \mathrm{CHFC}(=\mathrm{O}) \mathrm{OH}$ & 2,3,3,3-tetrafluoropropionic acid & $\mathrm{CH}_{3} \mathrm{CH}_{2} \mathrm{C}(=\mathrm{O}) \mathrm{O} \bullet$ & propionyloxyl radical \\
\hline $\mathrm{CF}_{3} \mathrm{CF}_{2} \mathrm{C}(=\mathrm{O}) \mathrm{OH}$ & 2,2,3,3,3-pentafluoropropionic acid & $\mathrm{CH}_{3} \mathrm{CHFC}(=\mathrm{O}) \mathrm{O} \bullet$ & 2-fluoropropionyloxyl radical \\
\hline $\mathrm{CH}_{2} \cdot \mathrm{C}(=\mathrm{O}) \mathrm{OH}$ & carboxymeth-1yl & $\mathrm{CH}_{3} \mathrm{CF}_{2} \mathrm{C}(=\mathrm{O}) \mathrm{O} \bullet$ & 2,2-difluoropropionyloxyl radical \\
\hline $\mathrm{CHF} \cdot \mathrm{C}(=\mathrm{O}) \mathrm{OH}$ & carboxy-1-fluorometh-1yl & $\mathrm{CH}_{2} \mathrm{FCH}_{2} \mathrm{C}(=\mathrm{O}) \mathrm{O} \bullet$ & 3-fluoropropionyloxyl radical \\
\hline $\mathrm{CF}_{2} \cdot \mathrm{C}(=\mathrm{O}) \mathrm{OH}$ & carboxy-1,1-difluorometh-1yl & $\mathrm{CH}_{2} \mathrm{FCHFC}(=\mathrm{O}) \mathrm{O} \bullet$ & 2,3-difluoropropionyloxyl radical \\
\hline $\mathrm{CH}_{3} \mathrm{C}(=\mathrm{O}) \mathrm{O} \bullet$ & acetyloxy radical & $\mathrm{CH}_{2} \mathrm{FCF}_{2} \mathrm{C}(=\mathrm{O}) \mathrm{O} \bullet$ & 2,2,3-trifluoropropionyloxyl radical \\
\hline
\end{tabular}




\begin{tabular}{|l|l|l|l|}
\hline $\mathrm{CH}_{2} \mathrm{FC}(=\mathrm{O}) \mathrm{O} \bullet$ & 2-fluoroacetyloxy radical & $\mathrm{CHF}_{2} \mathrm{CH}_{2} \mathrm{C}(=\mathrm{O}) \mathrm{O} \bullet$ & 3,3-difluoropropionyloxyl radical \\
\hline $\mathrm{CHF}_{2} \mathrm{C}(=\mathrm{O}) \mathrm{O} \bullet$ & 2,2-difluoroacetyloxy radical & $\mathrm{CHF}_{2} \mathrm{CHFC}(=\mathrm{O}) \mathrm{O} \bullet$ & 2,3,3-trifluoropropionyloxyl radical \\
\hline $\mathrm{CF}_{3} \mathrm{C}(=\mathrm{O}) \mathrm{O} \bullet$ & 2,2,2-trifluoroacetyloxy radical & $\mathrm{CHF}_{2} \mathrm{CF}_{2} \mathrm{C}(=\mathrm{O}) \mathrm{O} \bullet$ & 2,2,3,3-tetrafluoropropionyloxyl radical \\
\hline $\mathrm{CH}_{2} \bullet \mathrm{CH}_{2} \mathrm{C}(=\mathrm{O}) \mathrm{OH}$ & carboxyeth-2yl & $\mathrm{CF}_{3} \mathrm{CH}_{2} \mathrm{C}(=\mathrm{O}) \mathrm{O} \bullet$ & 3,3,3-trifluoropropionyloxyl radical \\
\hline $\mathrm{CH}_{2} \bullet \mathrm{CHFC}(=\mathrm{O}) \mathrm{OH}$ & carboxy-1-fluoroeth-2yl & $\left.\mathrm{CF}_{3} \mathrm{CHFC}_{2}=\mathrm{O}\right) \mathrm{O} \bullet$ & 2,3,3,3-tetrafluoropropionyloxyl radical \\
\hline $\mathrm{CH}_{2} \bullet \mathrm{CF}_{2} \mathrm{C}(=\mathrm{O}) \mathrm{OH}$ & carboxy-1,1-difluoroeth-2yl & $\mathrm{CF}_{3} \mathrm{CF}_{2} \mathrm{C}(=\mathrm{O}) \mathrm{O} \bullet$ & 2,2,3,3,3-pentafluoropropionyloxyl radical \\
\hline
\end{tabular}

${ }^{\mathrm{a}} \mathrm{Dot}(\bullet)$ represents a radical site on the preceding carbon or oxygen atom. 


\section{Parameters of fluorinated Carboxylic acids used as input to the Rotator.}

The torsional potential calculated at discrete torsional angles is represented by a truncated Fourier series: the symmetry and number of rotor are 3 and 1,respectively

$$
V(\phi)=a_{0}+a_{1} \cos (\phi)+a_{2} \cos (2 \phi)+a_{3} \cos (3 \phi)+b_{1} \sin (\phi)+b_{2} \sin (2 \phi)
$$

\begin{tabular}{|c|c|c|c|c|c|c|c|c|}
\hline \multirow{2}{*}{ Rotor } & \multirow{2}{*}{ 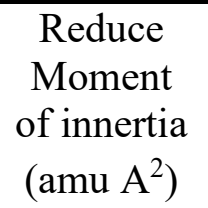 } & \multicolumn{7}{|c|}{ Fitted coefficients for the Fourier expansion } \\
\hline & & $\mathrm{a} 0$ & a1 & b1 & $\mathrm{a} 2$ & b2 & a3 & b3 \\
\hline \multicolumn{9}{|c|}{$\mathrm{CC}(=\mathrm{O}) \mathrm{OH}$} \\
\hline $\mathrm{C}-\mathrm{C}(=\mathrm{O}) \mathrm{OH}$ & 2.99 & 0.2429 & -0.0058 & -0.0089 & -0.0008 & 0.0019 & -0.2415 & 0.0136 \\
\hline $\mathrm{CC}(=\mathrm{O})--\mathrm{OH}$ & 0.85 & 7.1903 & -2.2437 & 0.0003 & -4.8215 & 0.0012 & -0.3681 & 0.0001 \\
\hline \multicolumn{9}{|c|}{$\mathrm{CFC}(=\mathrm{O}) \mathrm{OH}$} \\
\hline $\mathrm{CF}-\mathrm{C}(=\mathrm{O}) \mathrm{OH}$ & 93.88 & 1.5377 & 0.2382 & -0.0002 & -1.4098 & 0.0038 & -0.3231 & 0.0013 \\
\hline $\mathrm{CFC}(=\mathrm{O})--\mathrm{OH}$ & 12.64 & 5.4241 & 0.3152 & -0.0076 & -5.5032 & -0.0106 & -0.5626 & -0.0004 \\
\hline \multicolumn{9}{|c|}{$\mathrm{CF}_{2} \mathrm{C}(=\mathrm{O}) \mathrm{OH}$} \\
\hline $\mathrm{CF}_{2--\mathrm{C}}(=\mathrm{O}) \mathrm{OH}$ & 25.36 & 0.5801 & -0.2352 & -0.0015 & -0.0123 & 0.0034 & -0.3373 & 0.0050 \\
\hline $\mathrm{CF}_{2} \mathrm{C}(=\mathrm{O})--\mathrm{OH}$ & 0.86 & 5.4321 & -0.3925 & 0.0149 & -4.7141 & 0.4960 & -0.4034 & -0.0526 \\
\hline \multicolumn{9}{|c|}{$\mathrm{CF}_{3} \mathrm{C}(=\mathrm{O}) \mathrm{OH}$} \\
\hline $\mathrm{CF}_{3--} \mathrm{C}(=\mathrm{O}) \mathrm{OH}$ & 28.81 & 0.3976 & -0.0039 & -0.0068 & -0.0014 & 0.0026 & -0.3708 & 0.0057 \\
\hline $\mathrm{CF}_{3} \mathrm{C}(=\mathrm{O})--\mathrm{OH}$ & 0.85 & 5.6689 & -0.9786 & 0.0008 & -4.5658 & 0.0071 & -0.4481 & 0.0011 \\
\hline \multicolumn{9}{|c|}{$\mathrm{CCC}(=\mathrm{O}) \mathrm{OH}$} \\
\hline $\mathrm{C}-\mathrm{CC}(=\mathrm{O}) \mathrm{OH}$ & 3.12 & 1.0596 & 0.0062 & 0.0001 & -0.0081 & 0.0005 & -1.0619 & 0.0024 \\
\hline $\mathrm{CC}-\mathrm{C}(=\mathrm{O}) \mathrm{OH}$ & 19.69 & 1.0300 & -0.4113 & -0.0016 & -0.2844 & -0.0023 & -0.2877 & -0.0034 \\
\hline $\mathrm{CCC}(=\mathrm{O})--\mathrm{OH}$ & 0.86 & 7.0135 & -2.2103 & 0.1954 & -4.7329 & 0.4996 & -0.4011 & 0.0723 \\
\hline \multicolumn{2}{|c|}{$\mathrm{CCFC}(=\mathrm{O}) \mathrm{OH}$} & & & & & & & \\
\hline $\mathrm{C}-\mathrm{CFC}(=\mathrm{O}) \mathrm{OH}$ & 3.18 & 1.4218 & 0.0094 & 0.0080 & 0.0090 & -0.0069 & -1.4162 & -0.0410 \\
\hline
\end{tabular}




\begin{tabular}{|c|c|c|c|c|c|c|c|c|}
\hline CCF--C $(=\mathrm{O}) \mathrm{OH}$ & 25.99 & 0.9626 & -0.3377 & -0.3930 & -0.9045 & -0.0740 & 0.2919 & 0.1536 \\
\hline $\mathrm{CCFC}(=\mathrm{O})--\mathrm{OH}$ & 0.86 & 6.9659 & -2.6458 & -0.3545 & -4.2260 & -0.5649 & -0.3264 & -0.1299 \\
\hline \multicolumn{9}{|c|}{$\mathrm{CCF}_{2} \mathrm{C}(=\mathrm{O}) \mathrm{OH}$} \\
\hline $\mathrm{C}--\mathrm{CF}_{2} \mathrm{C}(=\mathrm{O}) \mathrm{OH}$ & 3.19 & 1.2577 & -0.0068 & 0.0108 & -0.0020 & -0.0041 & -1.3125 & -0.0551 \\
\hline 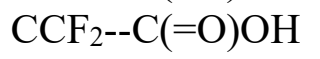 & 29.47 & 0.7827 & -0.5301 & 0.0488 & 0.1174 & -0.0225 & -0.3503 & 0.1000 \\
\hline $\mathrm{CCF}_{2} \mathrm{C}(=\mathrm{O})--\mathrm{OH}$ & 0.86 & 5.2523 & -0.0233 & -0.1682 & -4.7530 & -0.9075 & -0.3753 & -0.2929 \\
\hline \multicolumn{9}{|c|}{$\mathrm{CFCC}(=\mathrm{O}) \mathrm{OH}$} \\
\hline $\mathrm{CF}-\mathrm{CC}(=\mathrm{O}) \mathrm{OH}$ & 28.77 & 2.2671 & -0.6994 & -0.6265 & 0.6881 & -0.5291 & -1.5907 & 0.0501 \\
\hline $\mathrm{CFC}-\mathrm{C}(=\mathrm{O}) \mathrm{OH}$ & 32.06 & 1.4097 & -0.3185 & -0.1839 & -1.0591 & -0.4686 & -0.1199 & 0.2449 \\
\hline $\mathrm{CFCC}(=\mathrm{O})--\mathrm{OH}$ & 0.86 & 7.2494 & -2.2404 & 0.3155 & -4.7853 & -0.1681 & -0.3857 & -0.0406 \\
\hline \multicolumn{9}{|c|}{$\mathrm{CFCFC}(=\mathrm{O}) \mathrm{OH}$} \\
\hline $\mathrm{CF}-\mathrm{CFC}(=\mathrm{O}) \mathrm{OH}$ & 29.88 & 2.3259 & 0.3450 & -0.5778 & -1.2962 & -0.7302 & -1.4729 & 0.8026 \\
\hline $\mathrm{CFCF}-\mathrm{C}(=\mathrm{O}) \mathrm{OH}$ & 33.87 & 0.5686 & -0.1791 & 0.3242 & -0.3503 & 0.1860 & 0.0529 & -0.3199 \\
\hline $\mathrm{CFCFC}(=\mathrm{O})--\mathrm{OH}$ & 0.86 & 5.4384 & -0.3694 & -0.0032 & -4.7643 & -0.7792 & -0.4061 & -0.1563 \\
\hline
\end{tabular}




\begin{tabular}{|c|c|c|c|c|c|c|c|c|}
\hline \multicolumn{2}{|c|}{$\mathrm{CFCF}_{2} \mathrm{C}(=\mathrm{O}) \mathrm{OH}$} & \multirow[b]{2}{*}{2.3849} & \multirow[b]{2}{*}{-0.5759} & \multirow[b]{2}{*}{-0.9176} & \multirow[b]{2}{*}{0.0173} & \multirow[b]{2}{*}{-0.1684} & \multirow[b]{2}{*}{-1.8644} & \multirow[b]{2}{*}{0.0936} \\
\hline $\mathrm{CF}-\mathrm{CF}_{2} \mathrm{C}(=\mathrm{O}) \mathrm{OH}$ & 30.33 & & & & & & & \\
\hline $\mathrm{CFCF}_{2--\mathrm{C}}(=\mathrm{O}) \mathrm{OH}$ & 34.72 & 1.3158 & -0.4434 & 0.0753 & -1.1602 & 0.3800 & 0.2098 & -0.2805 \\
\hline $\mathrm{CFCF}_{2} \mathrm{C}(=\mathrm{O})--\mathrm{OH}$ & 0.86 & 5.4448 & -0.2521 & -0.0701 & -5.2547 & -0.3310 & -0.2296 & 0.1110 \\
\hline \multicolumn{2}{|c|}{$\mathrm{CF}_{2} \mathrm{CC}(=\mathrm{O}) \mathrm{OH}$} & & & & & & & \\
\hline $\mathrm{CF}_{2--\mathrm{CC}}(=\mathrm{O}) \mathrm{OH}$ & 45.82 & 2.8526 & -0.6208 & 0.7505 & 0.0723 & -0.1594 & -1.4959 & -0.5424 \\
\hline $\mathrm{CF}_{2} \mathrm{C}-\mathrm{-C}(=\mathrm{O}) \mathrm{OH}$ & 33.65 & 1.3623 & -0.3737 & 0.1061 & -0.9955 & 0.3382 & -0.0761 & -0.2124 \\
\hline $\mathrm{CF}_{2} \mathrm{CC}(=\mathrm{O})--\mathrm{OH}$ & 0.86 & 7.3096 & -2.6932 & 1.0611 & -4.1973 & 0.1059 & -0.7389 & 0.2977 \\
\hline \multicolumn{2}{|c|}{$\mathrm{CF}_{2} \mathrm{CFC}(=\mathrm{O}) \mathrm{OH}$} & & & & & & & \\
\hline $\mathrm{CF}_{2--\mathrm{CFC}}(=\mathrm{O}) \mathrm{OH}$ & 49.23 & 2.3062 & 0.0949 & 0.6661 & -0.8907 & 0.2664 & -1.5063 & -0.2633 \\
\hline $\mathrm{CF}_{2} \mathrm{CF}-\mathrm{C}(=\mathrm{O}) \mathrm{OH}$ & 34.91 & 2.2985 & -0.6300 & -0.0518 & -1.9627 & -0.1227 & 0.2849 & -0.0545 \\
\hline $\mathrm{CF}_{2} \mathrm{CFC}(=\mathrm{O})--\mathrm{OH}$ & 0.86 & 5.4084 & 0.3200 & -0.4447 & -5.5264 & -0.2189 & -0.4826 & 0.0298 \\
\hline \multicolumn{2}{|c|}{$\mathrm{CF}_{2} \mathrm{CF}_{2} \mathrm{C}(=\mathrm{O}) \mathrm{OH}$} & & & & & & & \\
\hline $\mathrm{CF}_{2}-\mathrm{CF}_{2} \mathrm{C}(=\mathrm{O}) \mathrm{OH}$ & 40.94 & 2.0610 & 0.1785 & -0.6335 & -0.3802 & -0.5292 & -1.7939 & 0.2641 \\
\hline $\mathrm{CF}_{2} \mathrm{CF}_{2}-\mathrm{C}(=\mathrm{O}) \mathrm{OH}$ & 35.72 & 1.4397 & -0.5364 & -0.0700 & -1.2482 & 0.1466 & 0.2603 & -0.2149 \\
\hline $\mathrm{CF}_{2} \mathrm{CF}_{2} \mathrm{C}(=\mathrm{O})--\mathrm{OH}$ & 0.86 & 5.4247 & -0.2260 & -0.1325 & -5.1410 & 0.4934 & -0.4048 & 0.1149 \\
\hline \multicolumn{2}{|c|}{$\mathrm{CF}_{3} \mathrm{CC}(=\mathrm{O}) \mathrm{OH}$} & & & & & & & \\
\hline $\mathrm{CF}_{3--\mathrm{CC}}(=\mathrm{O}) \mathrm{OH}$ & 56.15 & 1.6621 & -0.0515 & -0.0133 & -0.0294 & -0.0135 & -1.3008 & 0.3055 \\
\hline $\mathrm{CF}_{3} \mathrm{C}-\mathrm{-C}(=\mathrm{O}) \mathrm{OH}$ & 36.39 & 0.5251 & -0.0380 & 0.0018 & -0.2078 & 0.0264 & -0.2022 & 0.0398 \\
\hline $\mathrm{CF}_{3} \mathrm{CC}(=\mathrm{O})--\mathrm{OH}$ & 0.86 & 6.1740 & -1.1988 & -0.0566 & -4.6082 & -0.5027 & -0.5394 & -0.1813 \\
\hline \multicolumn{2}{|c|}{$\mathrm{CF}_{3} \mathrm{CFC}(=\mathrm{O}) \mathrm{OH}$} & & & & & & & \\
\hline $\mathrm{CF}_{3--\mathrm{CFC}}(=\mathrm{O}) \mathrm{OH}$ & 63.82 & 1.3492 & -0.0033 & 0.0021 & -0.0118 & -0.0005 & -1.4054 & 0.3063 \\
\hline $\mathrm{CF}_{3} \mathrm{CF}-\mathrm{C}(=\mathrm{O}) \mathrm{OH}$ & 37.22 & 0.9849 & 0.1791 & 0.0487 & -0.9418 & 0.0512 & -0.2608 & -0.0346 \\
\hline $\mathrm{CF}_{3} \mathrm{CFC}(=\mathrm{O})--\mathrm{OH}$ & 0.86 & 6.4426 & -1.8535 & 0.6590 & -4.4402 & -0.7498 & -0.3529 & -0.0250 \\
\hline \multicolumn{2}{|c|}{$\mathrm{CF}_{3} \mathrm{CF}_{2} \mathrm{C}(=\mathrm{O}) \mathrm{OH}$} & & & & & & & \\
\hline $\mathrm{CF}_{3}-\mathrm{CF}_{2} \mathrm{C}(=\mathrm{O}) \mathrm{OH}$ & 66.24 & 1.2456 & -0.0089 & 0.0013 & -0.0152 & 0.0034 & -1.4006 & -0.1997 \\
\hline $\mathrm{CF}_{3} \mathrm{CF}_{2}-\mathrm{C}(=\mathrm{O}) \mathrm{OH}$ & 37.66 & 0.3517 & 0.0476 & -0.1327 & -0.1971 & -0.1365 & -0.2300 & 0.1837 \\
\hline $\mathrm{CF}_{3} \mathrm{CF}_{2} \mathrm{C}(=\mathrm{O})-\mathrm{OH}$ & 0.86 & 5.6404 & -0.7749 & 0.2540 & -4.7792 & 0.2774 & -0.3205 & 0.1330 \\
\hline
\end{tabular}




\section{Parameters of fluorinated Carboxylic radicals used as input to the Rotator.}

The torsional potential calculated at discrete torsional angles is represented by a truncated Fourier series: the symmetry and number of rotor are 3 and 1 , respectively

$$
V(\phi)=a_{0}+a_{1} \cos (\phi)+a_{2} \cos (2 \phi)+a_{3} \cos (3 \phi)+b_{1} \sin (\phi)+b_{2} \sin (2 \phi)
$$

\begin{tabular}{|c|c|c|c|c|c|c|c|c|}
\hline \multirow{2}{*}{ Rotor } & \multirow{2}{*}{$\begin{array}{l}\text { Reduce } \\
\text { Moment of } \\
\text { innertia } \\
\left(\mathrm{amu} \mathrm{A} \mathrm{A}^{2}\right)\end{array}$} & \multicolumn{7}{|c|}{ Fitted coefficients for the Fourier expansion } \\
\hline & & $\mathrm{a} 0$ & a1 & b1 & a2 & $\mathrm{b} 2$ & a3 & b3 \\
\hline \multicolumn{9}{|c|}{$\mathrm{CjC}(=\mathrm{O}) \mathrm{OH}$} \\
\hline $\mathrm{Cj}-\mathrm{C}(=\mathrm{O}) \mathrm{OH}$ & 1.73 & 3.5146 & -0.1849 & -0.0172 & -3.8060 & -0.0027 & 0.2146 & 0.0174 \\
\hline $\mathrm{CjC}(=\mathrm{O})--\mathrm{OH}$ & 0.85 & 6.7163 & -2.4377 & -0.0004 & -4.0690 & -0.0015 & -0.3146 & -0.0002 \\
\hline \multicolumn{9}{|c|}{$\mathrm{CjFC}(=\mathrm{O}) \mathrm{OH}$} \\
\hline $\mathrm{CjF}-\mathrm{C}(=\mathrm{O}) \mathrm{OH}$ & 16.03 & 5.7238 & -0.6440 & -0.0379 & -5.9609 & -0.1170 & 0.5032 & 0.0714 \\
\hline $\mathrm{CjFC}(=\mathrm{O})--\mathrm{OH}$ & 0.86 & 5.7238 & -0.6440 & -0.0379 & -5.9609 & -0.1170 & 0.5032 & 0.0714 \\
\hline \multicolumn{9}{|c|}{$\mathrm{CjF}_{2} \mathrm{C}(=\mathrm{O}) \mathrm{OH}$} \\
\hline $\mathrm{CjF}_{2}-\mathrm{C}(=\mathrm{O}) \mathrm{OH}$ & 22.12 & 4.1991 & -0.1690 & -0.4422 & -4.2897 & -2.0040 & 0.1177 & 0.0558 \\
\hline $\mathrm{CjF}_{2} \mathrm{C}(=\mathrm{O})--\mathrm{OH}$ & 0.87 & 5.2390 & -1.7149 & 0.0011 & -3.4360 & 0.0041 & -0.1484 & 0.0002 \\
\hline \multicolumn{9}{|c|}{$\mathrm{CC}(=\mathrm{O}) \mathrm{Oj}$} \\
\hline $\mathrm{C}-\mathrm{C}(=\mathrm{O}) \mathrm{Oj}$ & 2.99 & 0.0313 & -0.0007 & 0.0008 & -0.0001 & 0.0004 & -0.0319 & -0.0102 \\
\hline \multicolumn{9}{|c|}{$\mathrm{CFC}(=\mathrm{O}) \mathrm{Oj}$} \\
\hline $\mathrm{CF}-\mathrm{-C}(=\mathrm{O}) \mathrm{Oj}$ & 18.00 & 1.7161 & -0.6530 & 0.0013 & -0.9905 & 0.0036 & -0.0844 & 0.0004 \\
\hline \multicolumn{9}{|c|}{$\mathrm{CF}_{2} \mathrm{C}(=\mathrm{O}) \mathrm{Oj}$} \\
\hline $\mathrm{CF}_{2}--\mathrm{C}(=\mathrm{O}) \mathrm{Oj}$ & 24.38 & 0.5230 & -0.7148 & 0.2617 & 0.2563 & -0.2195 & -0.0122 & 0.0300 \\
\hline \multicolumn{9}{|c|}{$\mathrm{CF}_{3} \mathrm{C}(=\mathrm{O}) \mathrm{Oj}$} \\
\hline $\mathrm{CF}_{3--\mathrm{C}}(=\mathrm{O}) \mathrm{Oj}$ & 27.49 & 0.0196 & -0.0002 & 0.0000 & 0.0000 & -0.0001 & 0.0005 & 0.0013 \\
\hline
\end{tabular}




\begin{tabular}{|c|c|c|c|c|c|c|c|c|}
\hline $\mathrm{Cj}-\mathrm{CC}(=\mathrm{O}) \mathrm{OH}$ & 1.76 & 0.5653 & -0.0180 & -0.0004 & -0.5200 & -0.0245 & -0.0137 & -0.0009 \\
\hline $\mathrm{CjC}-\mathrm{C}(=\mathrm{O}) \mathrm{OH}$ & 18.11 & 1.1021 & -0.4110 & 0.0214 & -0.3323 & 0.0276 & -0.3581 & 0.0486 \\
\hline $\mathrm{CjCC}(=\mathrm{O})--\mathrm{OH}$ & 0.86 & 6.4482 & -1.5465 & 0.5429 & -5.0284 & 0.0004 & -0.1712 & 0.1208 \\
\hline \multicolumn{9}{|c|}{$\mathrm{CjCFC}(=\mathrm{O}) \mathrm{OH}$} \\
\hline $\mathrm{Cj}-\mathrm{CFC}(=\mathrm{O}) \mathrm{OH}$ & 1.80 & 0.3896 & -0.0148 & -0.0201 & -0.3298 & -0.0152 & -0.0076 & 0.0165 \\
\hline $\mathrm{CjCF}-\mathrm{C}(=\mathrm{O}) \mathrm{OH}$ & 26.04 & 4.1829 & -2.7890 & -0.3579 & -1.7026 & -0.0377 & 0.1401 & 0.1356 \\
\hline $\mathrm{CjCFC}(=\mathrm{O})--\mathrm{OH}$ & 0.84 & 5.0833 & -0.6672 & 0.0124 & -5.1277 & 0.3488 & 0.6651 & -0.0925 \\
\hline \multicolumn{9}{|c|}{$\mathrm{CjCF}_{2} \mathrm{C}(=\mathrm{O}) \mathrm{OH}$} \\
\hline $\mathrm{Cj}--\mathrm{CF}_{2} \mathrm{C}(=\mathrm{O}) \mathrm{OH}$ & 1.80 & 0.5579 & -0.0314 & 0.0030 & -0.5955 & 0.1149 & 0.0023 & -0.0006 \\
\hline $\mathrm{CjCF}_{2}-\mathrm{C}(=\mathrm{O}) \mathrm{OH}$ & 29.09 & 0.7891 & -0.5829 & -0.0423 & 0.1170 & 0.0160 & -0.4074 & -0.0819 \\
\hline $\mathrm{CjCF}_{2} \mathrm{C}(=\mathrm{O})--\mathrm{OH}$ & 0.86 & 5.2441 & -0.1134 & -0.1358 & -4.7374 & -0.1302 & -0.3530 & -0.2830 \\
\hline \multicolumn{9}{|c|}{$\mathrm{CjFCC}(=\mathrm{O}) \mathrm{OH}$} \\
\hline $\mathrm{CjF}-\mathrm{CC}(=\mathrm{O}) \mathrm{OH}$ & 25.14 & 0.9921 & 0.0336 & 0.0764 & 0.1035 & 0.8402 & -0.1175 & -0.0657 \\
\hline $\mathrm{CjFC}-\mathrm{C}(=\mathrm{O}) \mathrm{OH}$ & 31.91 & 0.5788 & 0.1212 & 0.0860 & 987 & 0.2308 & -0.2651 & -0.1033 \\
\hline $\mathrm{CjFCC}(=\mathrm{O})--\mathrm{OH}$ & 0.86 & 6.1624 & -0.8340 & 0.4697 & -5.0698 & 0.2774 & -0.5854 & -0.3183 \\
\hline \multicolumn{9}{|c|}{$\mathrm{CjFCFC}(=\mathrm{O}) \mathrm{OH}$} \\
\hline $\mathrm{CjF}-\mathrm{CFC}(=\mathrm{O}) \mathrm{OH}$ & 24.88 & 1.9427 & -0.1492 & -0.0824 & -1.0721 & -1.0792 & -0.3758 & 0.5297 \\
\hline $\mathrm{CjFCF}-\mathrm{C}(=\mathrm{O}) \mathrm{OH}$ & 30.17 & 0.5259 & -0.3948 & 0.1366 & 0.1175 & -0.3432 & -0.2092 & 0.2662 \\
\hline $\mathrm{CjFCFC}(=\mathrm{O})--\mathrm{OH}$ & 0.84 & 4.9633 & -0.4738 & -0.3291 & -5.2158 & -0.1371 & 0.5691 & 0.0223 \\
\hline \multicolumn{9}{|c|}{$\mathrm{CjFCF}_{2} \mathrm{C}(=\mathrm{O}) \mathrm{OH}$} \\
\hline $\mathrm{CjF}--\mathrm{CF}_{2} \mathrm{C}(=\mathrm{O}) \mathrm{OH}$ & 25.94 & 1.4450 & 0.1725 & 0.1771 & 0.0531 & -0.3563 & -0.6579 & 0.3665 \\
\hline 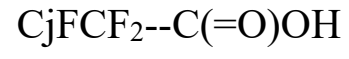 & 34.87 & 0.6342 & 0.1160 & 0.0206 & -0.4048 & 0.0981 & -0.3428 & 0.1059 \\
\hline $\mathrm{CjFCF}_{2} \mathrm{C}(=\mathrm{O})--\mathrm{OH}$ & 0.86 & 5.6508 & -0.8819 & -0.2341 & -4.6387 & 0.1642 & -0.3907 & 0.0007 \\
\hline \multicolumn{9}{|c|}{$\mathrm{CjF}_{2} \mathrm{CC}(=\mathrm{O}) \mathrm{OH}$} \\
\hline $\mathrm{CjF}_{2--\mathrm{CC}}(=\mathrm{O}) \mathrm{OH}$ & 41.14 & 1.1020 & -0.1433 & 0.2057 & 0.0757 & 0.6469 & -0.7659 & -0.5138 \\
\hline $\mathrm{CjF}_{2} \mathrm{C}-\mathrm{C}(=\mathrm{O}) \mathrm{OH}$ & 33.85 & 0.6818 & 0.0009 & 0.0108 & -0.4315 & 0.2230 & -0.2209 & -0.0885 \\
\hline $\mathrm{CjF}_{2} \mathrm{CC}(=\mathrm{O})--\mathrm{OH}$ & 0.86 & 6.5452 & -1.3868 & 0.3414 & -4.8700 & 0.2557 & -0.5959 & -0.2696 \\
\hline \multicolumn{9}{|c|}{$\mathrm{CjF}_{2} \mathrm{CFC}(=\mathrm{O}) \mathrm{OH}$} \\
\hline $\mathrm{CjF}_{2--\mathrm{CFC}}(=\mathrm{O}) \mathrm{OH}$ & 43.89 & 1.6869 & 0.1814 & -0.0653 & -0.9288 & -0.1830 & -0.9530 & 0.4155 \\
\hline $\mathrm{CjF}_{2} \mathrm{CF}-\mathrm{C}(=\mathrm{O}) \mathrm{OH}$ & 35.10 & 0.7276 & -0.1212 & 0.1155 & -0.5278 & 0.3167 & -0.0518 & -0.2722 \\
\hline
\end{tabular}




\begin{tabular}{|c|c|c|c|c|c|c|c|c|}
\hline $\mathrm{CjF}_{2} \mathrm{CFC}(=\mathrm{O})--\mathrm{OH}$ & 0.86 & 5.5331 & 0.0140 & -0.1935 & -5.0217 & -0.2034 & -0.4032 & -0.1252 \\
\hline \multicolumn{9}{|c|}{$\mathrm{CjF}_{2} \mathrm{CF}_{2} \mathrm{C}(=\mathrm{O}) \mathrm{OH}$} \\
\hline $\mathrm{CjF}_{2}-\mathrm{CF}_{2} \mathrm{C}(=\mathrm{O}) \mathrm{OH}$ & 45.33 & 1.3031 & -0.5318 & 0.0548 & -0.0133 & 0.0177 & -0.9513 & 0.3507 \\
\hline $\mathrm{CjF}_{2} \mathrm{CF}_{2--\mathrm{C}}(=\mathrm{O}) \mathrm{OH}$ & 47.32 & 0.7243 & 0.1535 & 0.1418 & -0.5262 & -0.1578 & -0.3288 & 0.0306 \\
\hline $\mathrm{CjF}_{2} \mathrm{CF}_{2} \mathrm{C}(=\mathrm{O})--\mathrm{OH}$ & 0.86 & 5.8039 & -0.6875 & -0.2731 & -4.7020 & -0.2212 & -0.5256 & 0.0720 \\
\hline \multicolumn{9}{|c|}{$\mathrm{CCjC}(=\mathrm{O}) \mathrm{OH}$} \\
\hline $\mathrm{C}--\mathrm{CjC}(=\mathrm{O}) \mathrm{OH}$ & 3.12 & 0.2049 & -0.0043 & 0.0076 & -0.0006 & -0.0013 & -0.1979 & -0.0111 \\
\hline $\mathrm{CCj}-\mathrm{C}(=\mathrm{O}) \mathrm{OH}$ & 17.54 & 4.6758 & -0.1988 & 0.0154 & -4.6774 & -0.0015 & -0.2407 & -0.0155 \\
\hline $\mathrm{CCjC}(=\mathrm{O})--\mathrm{OH}$ & 0.86 & 6.5409 & -2.3812 & 0.0002 & -3.9861 & 0.0005 & -0.2697 & 0.0000 \\
\hline \multicolumn{9}{|c|}{$\mathrm{CFCjC}(=\mathrm{O}) \mathrm{OH}$} \\
\hline $\mathrm{CF}-\mathrm{CjC}(=\mathrm{O}) \mathrm{OH}$ & 27.39 & 1.9816 & -2.2342 & -0.0013 & 0.4229 & 0.0005 & -0.1931 & -0.0004 \\
\hline $\mathrm{CFCj}-\mathrm{C}(=\mathrm{O}) \mathrm{OH}$ & 23.55 & 4.4629 & -0.1877 & -0.0501 & -4.5210 & -0.0239 & -0.1549 & 0.0421 \\
\hline $\mathrm{CFCjC}(=\mathrm{O})--\mathrm{OH}$ & 0.86 & 7.3486 & -3.5659 & -0.0007 & -3.5414 & -0.0013 & -0.3418 & -0.0002 \\
\hline \multicolumn{9}{|c|}{$\mathrm{CF}_{2} \mathrm{CjC}(=\mathrm{O}) \mathrm{OH}$} \\
\hline $\mathrm{CF}_{2--\mathrm{CjC}}(=\mathrm{O}) \mathrm{OH}$ & 43.22 & 2.6788 & -2.3440 & 0.0810 & -0.7651 & 0.0543 & 0.4431 & -0.0321 \\
\hline $\mathrm{CF}_{2} \mathrm{Cj}-\mathrm{C}(=\mathrm{O}) \mathrm{OH}$ & 32.48 & 4.0001 & -0.3660 & -0.0152 & -3.9451 & -0.2137 & -0.1077 & -0.0048 \\
\hline $\mathrm{CF}_{2} \mathrm{CjC}(=\mathrm{O})--\mathrm{OH}$ & 0.86 & 6.7633 & -1.2185 & -1.0472 & -3.9174 & -0.4688 & -0.4784 & -0.1108 \\
\hline \multicolumn{9}{|c|}{$\mathrm{CF}_{3} \mathrm{CjC}(=\mathrm{O}) \mathrm{OH}$} \\
\hline $\mathrm{CF}_{3}-\mathrm{CjC}(=\mathrm{O}) \mathrm{OH}$ & 54.49 & 0.8379 & -0.0016 & 0.0041 & -0.0113 & -0.0178 & -0.7566 & 0.0153 \\
\hline $\mathrm{CF}_{3} \mathrm{Cj}-\mathrm{C}(=\mathrm{O}) \mathrm{OH}$ & 35.86 & 3.1073 & -0.3162 & 0.0244 & -3.1988 & -0.0112 & 0.2518 & -0.0157 \\
\hline $\mathrm{CF}_{3} \mathrm{CjC}(=\mathrm{O})--\mathrm{OH}$ & 0.86 & 5.8464 & -1.5725 & -0.0039 & -3.9758 & 0.0116 & -0.4927 & -0.0122 \\
\hline \multicolumn{9}{|c|}{$\mathrm{CCjFC}(=\mathrm{O}) \mathrm{OH}$} \\
\hline $\mathrm{C}-\mathrm{CjFC}(=\mathrm{O}) \mathrm{OH}$ & 3.15 & 0.4390 & -0.0047 & 0.0081 & -0.0010 & -0.0017 & -0.4717 & -0.0141 \\
\hline $\mathrm{CCjF}-\mathrm{C}(=\mathrm{O}) \mathrm{OH}$ & 23.55 & 5.5273 & -0.2974 & 0.1238 & -5.9627 & -1.1907 & -0.1663 & -0.0682 \\
\hline $\mathrm{CCjFC}(=\mathrm{O})--\mathrm{OH}$ & 0.86 & 5.2333 & -0.9872 & 0.0000 & -4.1320 & 0.0000 & -0.1736 & 0.0000 \\
\hline \multicolumn{9}{|c|}{$\mathrm{CFCjFC}(=\mathrm{O}) \mathrm{OH}$} \\
\hline $\mathrm{CF}-\mathrm{CjFC}(=\mathrm{O}) \mathrm{OH}$ & 28.15 & 1.6792 & -0.7303 & 1.5036 & -0.9511 & -1.2095 & -0.0430 & 0.0094 \\
\hline $\mathrm{CFCjF}-\mathrm{C}(=\mathrm{O}) \mathrm{OH}$ & 30.45 & 4.7663 & -0.3416 & 0.1122 & -5.2703 & -0.3110 & -0.1550 & -0.0813 \\
\hline $\mathrm{CFCjFC}(=\mathrm{O})--\mathrm{OH}$ & 0.86 & 5.4473 & -1.0468 & -0.1732 & -4.2694 & 0.0567 & -0.2120 & 0.0059 \\
\hline \multicolumn{2}{|c|}{$\mathrm{CF}_{2} \mathrm{CjFC}(=\mathrm{O}) \mathrm{OH}$} & & & & & & & \\
\hline $\mathrm{CF}_{2--\mathrm{CjFC}}(=\mathrm{O}) \mathrm{OH}$ & 44.61 & 2.5371 & -1.6017 & -0.0278 & -1.1544 & -0.0393 & 0.1247 & 0.0055 \\
\hline
\end{tabular}




\begin{tabular}{|c|c|c|c|c|c|c|c|c|}
\hline $\mathrm{CF}_{2} \mathrm{CjF}-\mathrm{C}(=\mathrm{O}) \mathrm{OH}$ & 36.26 & 3.5504 & 0.3609 & 0.1371 & -4.1062 & -0.4207 & -0.2089 & -0.1321 \\
\hline $\mathrm{CF}_{2} \mathrm{CjFC}(=\mathrm{O})--\mathrm{OH}$ & 0.86 & 5.6661 & -1.4394 & 0.0000 & -4.0674 & 0.0000 & -0.2397 & 0.0000 \\
\hline \multicolumn{9}{|c|}{$\mathrm{CF}_{3} \mathrm{CjFC}(=\mathrm{O}) \mathrm{OH}$} \\
\hline $\mathrm{CF}_{3}-\mathrm{Cj} \mathrm{FC}(=\mathrm{O}) \mathrm{OH}$ & 53.07 & 0.3266 & 0.0026 & 0.0000 & 0.0065 & -0.0005 & -0.2783 & 0.0352 \\
\hline $\mathrm{CF}_{3} \mathrm{CjF}-\mathrm{C}(=\mathrm{O}) \mathrm{OH}$ & 36.69 & 3.3378 & 0.1214 & 0.1928 & -3.8835 & -0.2978 & -0.1953 & -0.1448 \\
\hline $\mathrm{CF}_{3} \mathrm{CjFC}(=\mathrm{O})--\mathrm{OH}$ & 0.86 & 5.6533 & -1.4489 & -0.0026 & -4.0607 & -0.0146 & -0.2365 & -0.0012 \\
\hline \multicolumn{9}{|c|}{$\mathrm{CCC}(=\mathrm{O}) \mathrm{Oj}$} \\
\hline $\mathrm{C}-\mathrm{CC}(=\mathrm{O}) \mathrm{Oj}$ & 19.14 & 0.9514 & -0.0026 & 0.0000 & -0.0010 & 0.0000 & -1.0091 & 0.0057 \\
\hline $\mathrm{CC}-\mathrm{C}(=\mathrm{O}) \mathrm{Oj}$ & 19.14 & 0.7585 & -0.5931 & 0.0417 & -0.2456 & 0.0349 & 0.0967 & -0.0209 \\
\hline \multicolumn{9}{|c|}{$\mathrm{CCFC}(=\mathrm{O}) \mathrm{Oj}$} \\
\hline $\mathrm{C}-\mathrm{CFC}(=\mathrm{O}) \mathrm{Oj}$ & 3.17 & 1.3096 & 0.0077 & -0.0112 & 0.0003 & 0.0072 & -1.3464 & -0.0015 \\
\hline $\mathrm{CCF}-\mathrm{-C}(=\mathrm{O}) \mathrm{Oj}$ & 24.72 & 1.4548 & 0.5546 & 0.5783 & -0.9492 & 0.2490 & -0.0562 & 0.1358 \\
\hline \multicolumn{9}{|c|}{$\mathrm{CCF}_{2} \mathrm{C}(=\mathrm{O}) \mathrm{Oj}$} \\
\hline $\mathrm{C}--\mathrm{CF}_{2} \mathrm{C}(=\mathrm{O}) \mathrm{Oj}$ & 3.19 & 1.1063 & -0.0143 & -0.0023 & -0.0046 & -0.0047 & -1.1661 & 0.2401 \\
\hline $\mathrm{CCF}_{2--\mathrm{C}}(=\mathrm{O}) \mathrm{Oj}$ & 28.14 & 0.8022 & -0.9587 & -0.3858 & 0.1976 & 0.1865 & 0.0015 & 0.0021 \\
\hline \multicolumn{9}{|c|}{$\mathrm{CFCC}(=\mathrm{O}) \mathrm{Oj}$} \\
\hline $\mathrm{CF}-\mathrm{CC}(=\mathrm{O}) \mathrm{Oj}$ & 28.95 & 1.8347 & 0.5608 & -0.9997 & -0.6005 & -0.7528 & -1.1654 & 0.6205 \\
\hline $\mathrm{CFC}-\mathrm{C}(=\mathrm{O}) \mathrm{Oj}$ & 30.60 & 1.3161 & -0.6400 & -0.4689 & -0.6516 & 0.1172 & -0.0736 & 0.1610 \\
\hline \multicolumn{9}{|c|}{$\mathrm{CFCFC}(=\mathrm{O}) \mathrm{Oj}$} \\
\hline $\mathrm{CF}-\mathrm{CFC}(=\mathrm{O}) \mathrm{Oj}$ & 29.80 & 2.9603 & 0.8552 & -0.3879 & -1.1827 & 0.3774 & -1.5300 & -0.6178 \\
\hline $\mathrm{CFCF}-\mathrm{C}(=\mathrm{O}) \mathrm{Oj}$ & 31.82 & 2.7105 & -1.1641 & -0.6576 & -1.6021 & 0.1131 & 0.0512 & 0.2114 \\
\hline \multicolumn{9}{|c|}{$\mathrm{CFCF}_{2} \mathrm{C}(=\mathrm{O}) \mathrm{Oj}$} \\
\hline $\mathrm{CF}-\mathrm{CF}_{2} \mathrm{C}(=\mathrm{O}) \mathrm{Oj}$ & 30.40 & 0.0276 & -0.0096 & 0.0205 & 0.0071 & 0.0084 & -0.0021 & -0.0002 \\
\hline $\mathrm{CFCF}_{2--} \mathrm{C}(=\mathrm{O}) \mathrm{Oj}$ & 32.97 & 1.1268 & -1.0732 & 0.7270 & -0.0881 & 0.0391 & 0.1932 & -0.2106 \\
\hline \multicolumn{9}{|c|}{$\mathrm{CF}_{2} \mathrm{CC}(=\mathrm{O}) \mathrm{Oj}$} \\
\hline $\mathrm{CF}_{2--} \mathrm{CC}(=\mathrm{O}) \mathrm{Oj}$ & 46.64 & 2.0628 & -0.7858 & 1.0567 & 0.1850 & 0.3094 & -1.4305 & -0.2989 \\
\hline $\mathrm{CF}_{2} \mathrm{C}-\mathrm{-C}(=\mathrm{O}) \mathrm{Oj}$ & 33.23 & 0.7507 & -0.5911 & -0.5003 & 0.0238 & 0.1415 & -0.0609 & 0.1024 \\
\hline \multicolumn{9}{|c|}{$\mathrm{CF}_{2} \mathrm{CFC}(=\mathrm{O}) \mathrm{Oj}$} \\
\hline $\mathrm{CF}_{2--\mathrm{CFC}}(=\mathrm{O}) \mathrm{Oj}$ & 48.89 & 0.5554 & 0.4380 & 0.7997 & -0.1571 & 0.5233 & -0.3187 & 0.2930 \\
\hline $\mathrm{CF}_{2} \mathrm{CF}-\mathrm{C}(=\mathrm{O}) \mathrm{Oj}$ & 34.17 & 1.7687 & -0.1049 & -0.6939 & -1.3890 & 0.8326 & -0.1441 & 0.1091 \\
\hline
\end{tabular}




\begin{tabular}{|c|c|c|c|c|c|c|c|c|}
\hline $\mathrm{CF}_{2}-\mathrm{CF}_{2} \mathrm{C}(=\mathrm{O}) \mathrm{Oj}$ & 50.43 & 1.7598 & -0.1564 & 0.0130 & 0.2971 & -0.0404 & -1.6585 & 0.3750 \\
\hline $\mathrm{CF}_{2} \mathrm{CF}_{2}-\mathrm{C}(=\mathrm{O}) \mathrm{Oj}$ & 34.99 & 0.6704 & -0.1766 & 0.6810 & -0.0079 & 0.3294 & -0.3255 & -0.1409 \\
\hline \multicolumn{9}{|c|}{$\mathrm{CF}_{3} \mathrm{CC}(=\mathrm{O}) \mathrm{Oj}$} \\
\hline $\mathrm{CF}_{3--\mathrm{CC}}(=\mathrm{O}) \mathrm{Oj}$ & 58.44 & 1.5360 & 0.0015 & 0.0050 & -0.0082 & 0.0066 & -1.4574 & 0.0616 \\
\hline $\mathrm{CF}_{3} \mathrm{C}-\mathrm{-C}(=\mathrm{O}) \mathrm{Oj}$ & 33.78 & 0.3502 & -0.1841 & 0.1598 & -0.0059 & 0.0345 & -0.0461 & -0.0728 \\
\hline \multicolumn{9}{|c|}{$\mathrm{CF}_{3} \mathrm{CFC}(=\mathrm{O}) \mathrm{Oj}$} \\
\hline $\mathrm{CF}_{3}-\mathrm{CFC}(=\mathrm{O}) \mathrm{Oj}$ & 63.49 & 1.2545 & -0.4597 & 0.5875 & 0.1613 & 0.6384 & -1.1316 & 0.4577 \\
\hline $\mathrm{CF}_{3} \mathrm{CF}-\mathrm{C}(=\mathrm{O}) \mathrm{Oj}$ & 35.05 & 1.1758 & -0.3177 & 0.2479 & -0.8760 & 0.0497 & 0.0751 & -0.0084 \\
\hline \multicolumn{9}{|c|}{$\mathrm{CF}_{3} \mathrm{CF}_{2} \mathrm{C}(=\mathrm{O}) \mathrm{Oj}$} \\
\hline $\mathrm{CF}_{3}--\mathrm{CF}_{2} \mathrm{C}(=\mathrm{O}) \mathrm{Oj}$ & 66.09 & 1.4465 & -0.0061 & -0.0032 & -0.0168 & 0.0032 & -1.5420 & -0.0742 \\
\hline $\mathrm{CF}_{3} \mathrm{CF}_{2--\mathrm{C}}(=\mathrm{O}) \mathrm{Oj}$ & 35.40 & 0.4640 & -0.0098 & 0.1129 & -0.4783 & -0.0833 & 0.0277 & -0.1047 \\
\hline
\end{tabular}

Note: $\mathrm{j}$ is represented the radical site in molecules 


\section{Complete References}

1. Chase, M. W., NIST-JANAF Thermochemical Tables for Oxygen Fluorides. Journal of Physical and Chemical Reference Data 1996, 25 (2), 551-603.

2. Ruscic, B.; Bross, D. H. Active Thermochemical Tables (ATcT) values based on ver. 1.122g of the Thermochemical Network. https://ATcT.anl.gov.

3. Manion, J. A., Evaluated Enthalpies of Formation of the Stable Closed Shell C1 and C2 Chlorinated Hydrocarbons. Journal of Physical and Chemical Reference Data 2002, 31 (1), 123-172.

4. Wang, H.; Castillo, Á.; Bozzelli, J. W., Thermochemical Properties Enthalpy, Entropy, and Heat Capacity of C1-C4 Fluorinated Hydrocarbons: Fluorocarbon Group Additivity. J. Phys. Chem. A 2015, 119 (29), 8202-8215.

5. Pittam, D. A.; Pilcher, G., Measurements of heats of combustion by flame calorimetry. Part 8.-Methane, ethane, propane, n-butane and 2-methylpropane. Journal of the Chemical Society, Faraday Transactions 1: Physical Chemistry in Condensed Phases 1972, 68 (0), $2224-2229$.

6. Nagy, B.; Csontos, B.; Csontos, J.; Szakács, P.; Kállay, M., High-Accuracy Theoretical Thermochemistry of Fluoroethanes. J. Phys. Chem. A 2014, 118 (26), 4824-4836.

7. Prosen, E. J.; Rossini, F. D., Heats of combustion and formation of the paraffin hydrocarbons at 25 C. J. Res. NBS 1945, $34,263-267$.

8. Csontos, J.; Rolik, Z.; Das, S.; Kállay, M., High-Accuracy Thermochemistry of Atmospherically Important Fluorinated and Chlorinated Methane Derivatives.J. Phys. Chem. A 2010, 114 (50), 13093-13103.

9. Chen, S. S.; Rodgers, A. S.; Choo, J.; Wilhoit, R. C.; Zwolinski, B. J., Ideal gas thermodynamic properties of six fluoroethanes. J. Phys. Chem. Ref. Data 1975, 4 (2), 441-456.

10. Goldsmith, C. F.; Magoon, G. R.; Green, W. H., Database of Small Molecule Thermochemistry for Combustion. J. Phys. Chem. A 2012, 116 (36), 9033 9057.

11. Zachariah, M. R.; Westmoreland, P. R.; Burgess, D. R.; Tsang, W.; Melius, C. F., BAC-MP4 Predictions of Thermochemical Data for C1 and C2 Stable and Radical Hydrofluorocarbons and Oxidized Hydrofluorocarbons. J. Phys. Chem. 1996, 100 (21), 8737-8747.

12. Berry, R. J.; Burgess, D. R. F.; Nyden, M. R.; Zachariah, M. R.; Schwartz, M., Halon Thermochemistry: Ab Initio Calculations of the Enthalpies of Formation of Fluoromethanes. The Journal of Physical Chemistry 1995, 99 (47), 17145-17150.

13. Pedley, J. B.; Naylor, R. D.; Kirby, S. P.; Pedley, J. B., Thermochemical data of organic compounds. Chapman and Hall: London; New York, 1986.

14. Atkinson, R.; Baulch, D. L.; Cox, R. A.; Hampson, R. F.; Kerr, J. A.; Rossi, M. J.; Troe, J., Evaluated Kinetic and Photochemical Data for Atmospheric Chemistry, Organic Species: Supplement VII. Journal of Physical and Chemical Reference Data 1999, 28 (2), $191-393$.

15. Lacher, J. R.; Skinner, H. A., Bond energy additivity and bond interactions in fluoro-halogenated hydrocarbons. Journal of the Chemical Society A: Inorganic, Physical, Theoretical 1968, (0), 1034-1038.

16. Haworth, N. L.; Smith, M. H.; Bacskay, G. B.; Mackie, J. C., Heats of Formation of Hydrofluorocarbons Obtained by Gaussian-3 and Related Quantum Chemical Computations. J. Phys. Chem. A 2000, 104 (32), 7600-7611.

17. Tsang, W., Heats of Formation of Organic Free Radicals by Kinetic Methods. In Energetics of Organic Free Radicals, Martinho Simões, J. A.; Greenberg, A.; Liebman, J. F., Eds. Springer Netherlands: Dordrecht, 1996; pp 22-58.

18. Kormos, B.; Liebman, J.; Cramer, C., $298 \mathrm{~K}$ enthalpies of formation of monofluorinated alkanes: Theoretical predictions for methyl, ethyl, isopropyl and tert-butyl fluoride. J. Phys. Org. Chem. 2004, 17, 656-664.

19. Stull, D. R.; Sinke, G. C.; Westrum, E. F., The chemical thermodynamics of organic compounds. Krieger: Malabar, Fla, 1987.

20. Espinosa-García, J.; Dóbé, S., Theoretical enthalpies of formation for atmospheric hydroxycarbonyls. Journal of Molecular Structure: THEOCHEM 2005, $713(1), 119-125$ 
21. Purnell, D. L.; Bozzelli, J. W., Thermochemical Properties: Enthalpy, Entropy, and Heat Capacity of C2-C3 Fluorinated Aldehydes. Radicals and Fluorocarbon Group Additivity. The Journal of Physical Chemistry A 2019, 123 (3), 650-665.

22. Luo, Y.-R.; Benson, S. W., Heats of Formation of Alkyl Fluorides. J. Phys. Chem. A 1997, 101 (16), 3042-3044.

23. Sun, H.; Bozzelli, J. W., Structures, Intramolecular Rotation Barriers, and Thermochemical Properties: Ethanol, $\alpha$-Monoethanols, Dichloroethanols, and Corresponding Radicals Derived from H Atom Loss. J. Phys. Chem. A 2001, 105 (41), 9543-9552.

24. Kraka, E.; Konkoli, Z.; Cremer, D.; Fowler, J.; Schaefer, H. F., Difluorodioxirane: An Unusual Cyclic Peroxide. J. Am. Chem. Soc. 1996, 118 (43), $10595-$ 10608.

25. Selected Values of Properties of Chemical Compounds. In NIST Chemistry Webbook, Thermodynamics Research Center, Texas A\&M University, College Station, Texas: 1997.

26. Cox, J. D.; Wagman, D. D.; Medvedev, V. A., CODATA key values for thermodynamics. Hemisphere Pub. Corp.: New York, 1989.

27. Ganyecz, Á.; Kállay, M.; Csontos, J., Accurate Theoretical Thermochemistry for Fluoroethyl Radicals. J. Phys. Chem. A 2017, 121 (5), $1153-1162$.

28. Snitsiriwat, S.; Yommee, S.; Bozzelli, J. W., Thermochemistry of Intermediates and Products in the Oxidation Reaction of 1,1,2-Trifluoroethene via OH Radical. J. Phys. Chem. A 2019, 123 (37), 8017-8027.

29. Wang, H.; Bozzelli, J. W., Thermochemical Properties and Bond Dissociation Energies for Fluorinated Methanol, $\mathrm{CH} 3-\mathrm{xFxOH}$, and Fluorinated Methyl Hydroperoxides, CH3-xFxOOH: Group Additivity. J. Phys. Chem. A 2016, 120 (35), 6998-7010.

30. Wiberg, K. B.; Crocker, L. S.; Morgan, K. M., Thermochemical studies of carbonyl compounds. 5. Enthalpies of reduction of carbonyl groups. Journal of the American Chemical Society 1991, 113 (9), 3447-3450.

31. Marshall, P., Thermochemistry of the Ethyl Radical and the C-H Bond Strength in Ethane. The Journal of Physical Chemistry A 1999, 103 (23), $4560-4563$.

32. Frisch, M. J., Trucks, G. W., Schlegel, H. B., Scuseria, G. E., Robb, M. A., Cheeseman, J. R., Scalmani, G., Barone, V., Petersson, G. A., Nakatsuji, H., Li, X., Caricato, M., Marenich, A. V., Bloino, J., Janesko, B. G., Gomperts, R., Mennucci, B., Hratchian, H. P., Ortiz, J. V., Izmaylov, A. F., Sonnenberg, J. L., Williams-Young, D., Ding, F., Lipparini, F., Egidi, F., Goings, J., Peng, B., Petrone, A., Henderson, T., Ranasinghe, D., Zakrzewski, V. G., Gao, J., Rega, N., Zheng, G., Liang, W., Hada, M., Ehara, M., Toyota, K., Fukuda, R., Hasegawa, J., Ishida, M., Nakajima, T., Honda, Y., Kitao, O., Nakai, H., Vreven, T., Throssell, K., Montgomery, Jr., J. A., Peralta, J. E., Ogliaro, F., Bearpark, M. J., Heyd, J. J., Brothers, E. N., Kudin, K. N., Staroverov, V. N., Keith, T. A., Kobayashi, R., Normand, J., Raghavachari, K., Rendell, A. P., Burant, J. C., Iyengar, S. S., Tomasi, J., Cossi, M., Millam, J. M., Klene, M., Adamo, C., Cammi, R., Ochterski, J. W., Martin, R. L., Morokuma, K., Farkas, O., Foresman, J. B., Fox, D. J. Gaussian 16, Revision A.03, Gaussian, Inc.: Wallingford, CT, 2016. 